Florida International University

FIU Digital Commons

FIU Electronic Theses and Dissertations

University Graduate School

6-12-2019

\title{
Essays in Real Estate Securities and Financial Institutions
}

Zifeng Feng

zfeng@fiu.edu

Follow this and additional works at: https://digitalcommons.fiu.edu/etd

Part of the Corporate Finance Commons, and the Real Estate Commons

\section{Recommended Citation}

Feng, Zifeng, "Essays in Real Estate Securities and Financial Institutions" (2019). FIU Electronic Theses and Dissertations. 4264.

https://digitalcommons.fiu.edu/etd/4264

This work is brought to you for free and open access by the University Graduate School at FIU Digital Commons. It has been accepted for inclusion in FIU Electronic Theses and Dissertations by an authorized administrator of FIU Digital Commons. For more information, please contact dcc@fiu.edu. 


\title{
FLORIDA INTERNATIONAL UNIVERSITY
}

\author{
Miami, Florida
}

\section{ESSAYS IN REAL ESTATE SECURITIES AND FINANCIAL INSTITUTIONS}

A dissertation submitted in partial fulfillment of

the requirements for the degree of

DOCTOR OF PHILOSOPHY

in

BUSINESS ADMINISTRATION

by

Zifeng Feng 
To: Dean Joanne Li

College of Business

This dissertation, written by Zifeng Feng, and entitled Essays in Real Estate Securities and Financial Institutions, having been approved in respect to style and intellectual content, is referred to you for judgment.

We have read this dissertation and recommend that it be approved.

Abhijit Barua

Eli Beracha

William Hardin

Xiaoquan Jiang

Zhonghua Wu, Major Professor

Date of Defense: June 12, 2019

The dissertation of Zifeng Feng is approved.

Dean Joanne Li

College of Business

Andrés G. Gil

Vice President for Research and Economic Development and Dean of the University Graduate School

Florida International University, 2019 
C Copyright 2019 by Zifeng Feng

All rights reserved. 


\section{DEDICATION}

I want to dedicate this dissertation to all members of my family, especially my wife, Yanfang Qin. Without her support and encouragement, I would not be able to complete this work. 


\section{ACKNOWLEDGMENTS}

I want to thank Abhijit Barua, Eli Beracha, William Hardin, Xiaoquan Jiang, and Zhonghua $\mathrm{Wu}$, members of the dissertation committee for their helpful suggestions and continued support. I want to express my special gratitude to my advisor Zhonghua Wu for his kind guidance, valuable feedback, incredible support and inspiration over the years. I sincerely appreciate the valuable time and expertise he has contributed. I also like to express my special gratitude to William Hardin, who has helped me a lot and encouraged me to exceed the expectations in the past years. 


\title{
ABSTRACT OF THE DISSERTATION
}

\section{ESSAYS IN REAL ESTATE SECURITIES AND FINANCIAL INSTITUTIONS}

\author{
by \\ Zifeng Feng \\ Florida International University, 2019 \\ Miami, Florida \\ Professor Zhonghua Wu, Major Professor
}

This dissertation consists of three essays that examine aspects of real estate securities and financial institutions.

The first essay examines the relations between Real Estate Investment Trust (REIT) efficiency and operational performance, risk, and stock return. REIT-level operational efficiency is measured as the ratio of operational expenses to revenue, where a higher operational efficiency ratio (OER) indicates a less efficient REIT. For a sample of U.S. equity REITs, operational performance, measured by return on assets as well as return on equity, is negatively associated with previous-year operational efficiency ratios. Results further show that more efficient REITs have lower levels of credit risk and total risk. Perhaps most important, empirical evidence shows that the cross-sectional stock return of REITs is partially explained by operational efficiency and that a portfolio consisting of 
highly efficient REITs earns, on average, a higher cumulative stock return than a portfolio consisting of low efficiency REITs.

The second essay analyzes the impact of technology investment on firm performance and market value using a unique dataset on technology spending by U.S. banks. It first documents that banks increasingly invested in technology from 2000-2017 and did not cut technology spending even when experiencing negative performance shocks. Meanwhile, operating performance and market value are positively correlated with lagged technology spending, and the positive correlation is primarily driven by large banks. Interestingly, while technology spending increases asset turnover, it only improves the profit margin for large banks.

The third essay investigates the impact of technology investment on bank production and employment. It documents that technology input on average contributes about $12.85 \%$ to the increase in value-added output of banks from 2000-2017, according to the estimation from a firm-level production function correcting for endogenous input choices. Moreover, bank employment and tasks are positively correlated with their lagged technology spending in the cross-section, supporting the task-based framework of Acemoglu and Restrepo (2018). These results indicate that technology capital is highly productive to US banks and use of technology generally lead to more bank employment at the firm-level, which is likely due to an increased amount of tasks created by new technology. 


\section{TABLE OF CONTENTS}

1. Chapter I: REIT Operational Efficiency: Performance, Risk, and Return .....................1

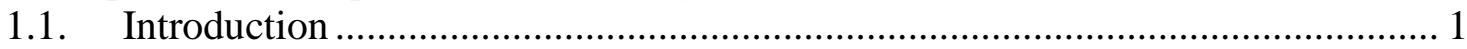

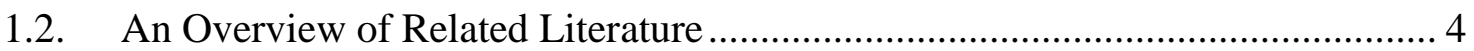

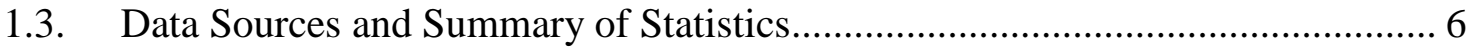

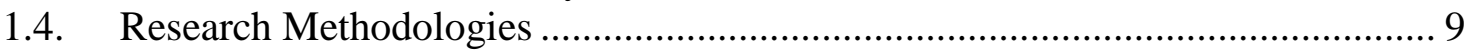

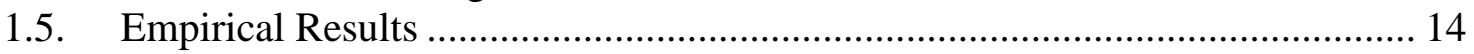

1.5.1. Operational Performance and Operational Efficiency................................... 14

1.5.2. Firm Risk and Operational Efficiency ....................................................... 17

1.5.3. Stock Return and Operational Efficiency ………......................................... 20

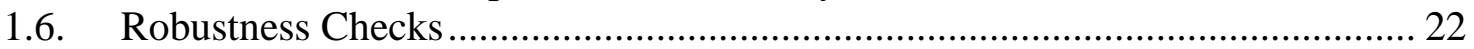

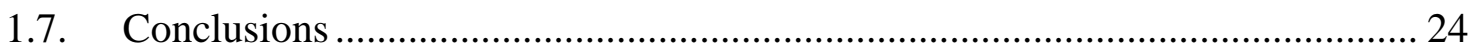

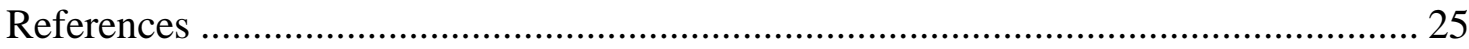

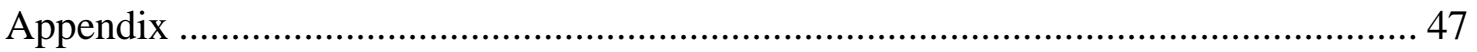

2. Chapter II: Bank Technology, Performance and Market Value....................................49

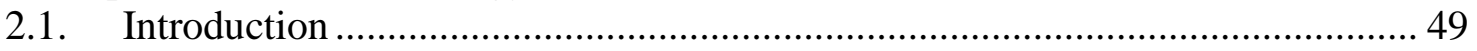

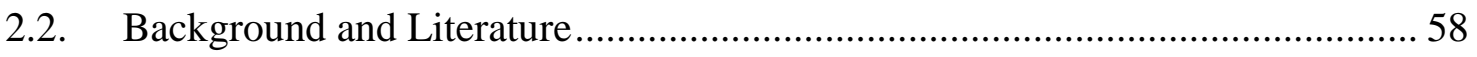

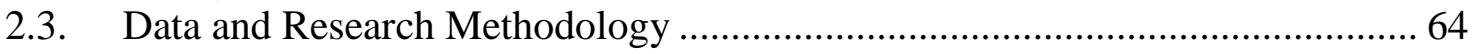

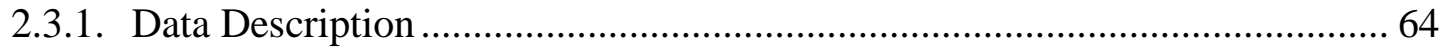

2.3.2. Empirical Specifications for the Correlation between Performance and

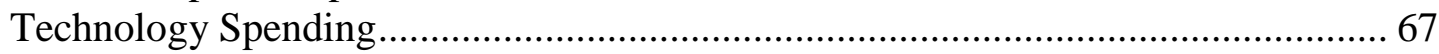

2.3.3. Firm Value and Technology …………………..................................... 70

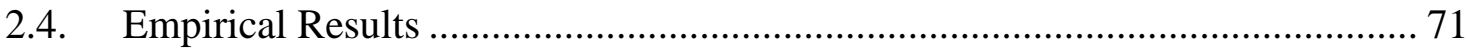

2.4.1. The Growth of Technology Spending and Its Relationship with Firm

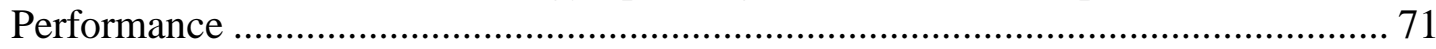

2.4.2. Firm Performance and Technology Spending .............................................. 73

2.4.3. Channel of Technology Spending Influencing Firm Performance.................. 75

2.4.4. Market Value and Technology Investment ……………………………....... 77

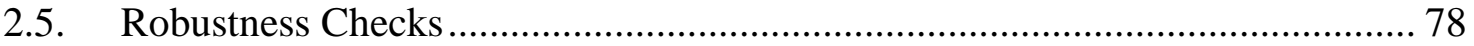

2.5.1. Alternative Technology Spending Levels .................................................... 78

2.5.2. Large Banks with Too-Big-To-Fail (TBTF) Banks Excluded ....................... 79

2.5.3. Large Banks without Mergers and Acquisitions Activities.............................. 79

2.5.4. Long Lags of Technology Spending Measures ............................................. 80

2.5.5. Further Discussion on Endogeneity between Firm Performance and

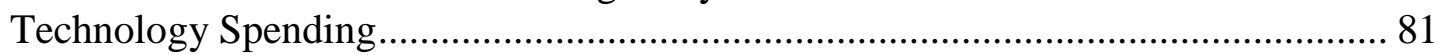

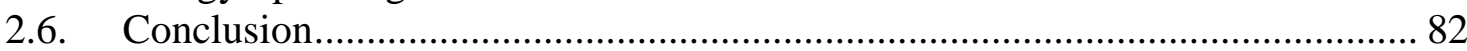

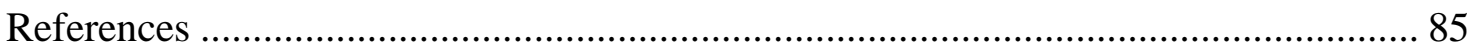

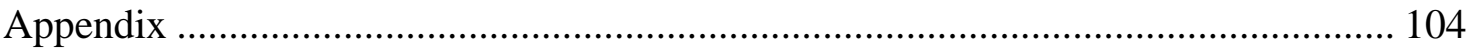


3. Chapter III: Bank Technology: Productivity and Employment …………………......111

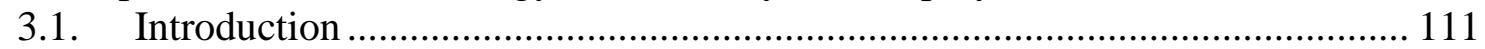

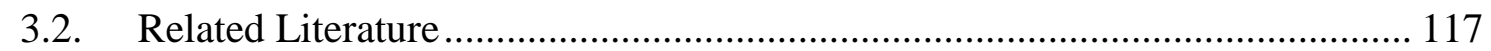

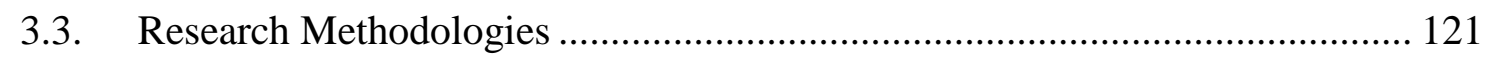

3.3.1. Technology and Production..................................................................... 121

3.3.2. Technology and Employment................................................................. 127

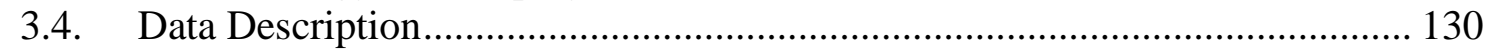

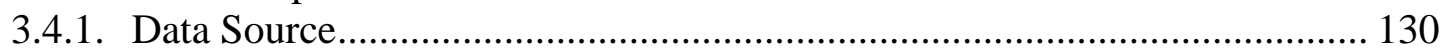

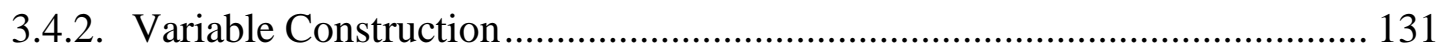

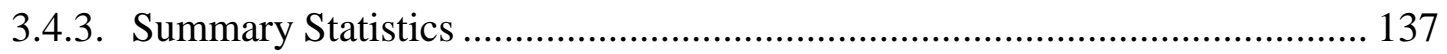

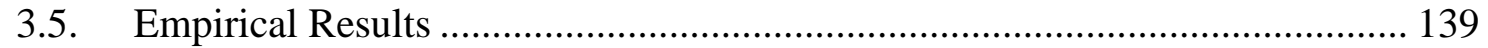

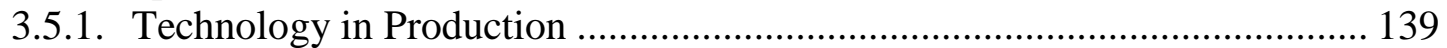

3.5.2. Technology and Employment................................................................. 142

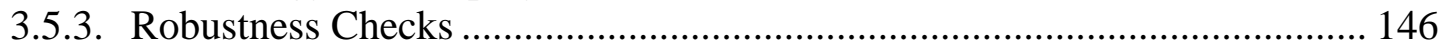

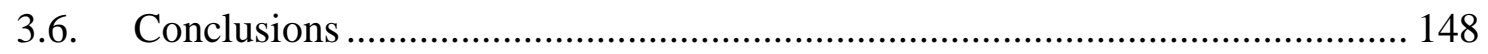

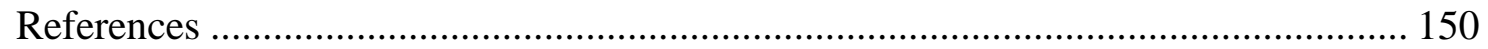

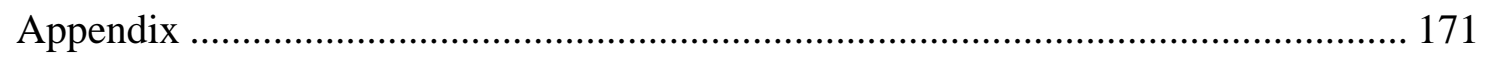

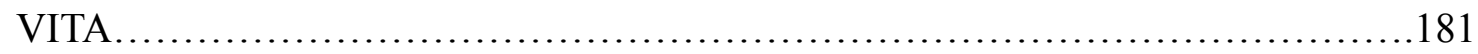




\section{LIST OF TABLES}

TABLE

PAGE

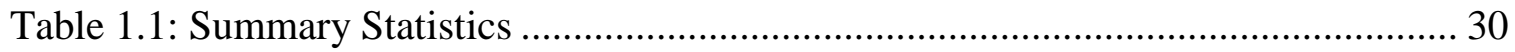

Table 1.2: Return on Assets and Operational Efficiency .................................................. 31

Table 1.3: Return on Equity and Operational Efficiency ............................................... 33

Table 1.4: Total Risk and Operational Efficiency ………………….............................. 35

Table 1.5: Credit Risk and Operational Efficiency...................................................... 37

Table 1.6: Cross-Sectional Stock Return and Operational Efficiency.............................. 39

Table 1.7: Residual Stock Return and Operational Efficiency .......................................... 40

Table 1.8: Robustness check ......................................................................................... 41

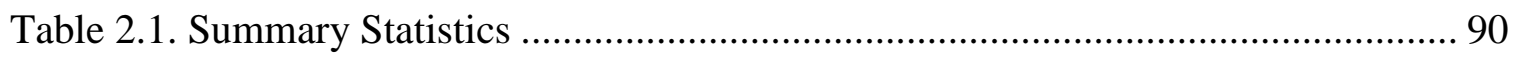

Table 2.2. Return on Assets and Technology Investment .............................................. 91

Table 2.3. Return on Equity and Technology Investment ............................................ 92

Table 2.4. Profit Margin and Technology Investment ................................................... 93

Table 2.5. Asset Turnover and Technology Investment .................................................. 94

Table 2.6. Market-to-Book Equity Ratio and Technology Investment ........................... 95

Table 2.7. Firm $Q$ and Technology Investment ............................................................ 96

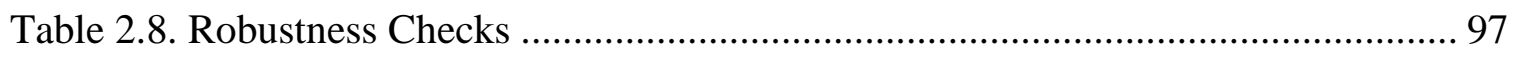

Table 3.1. Summary Statistics …………………………..................................... 157

Table 3.2. Production Function Parameter Estimates I.................................................. 158

Table 3.3. Production Function Parameter Estimates II ................................................. 159

Table 3.4. Persistence in Technology Spending and Employment................................ 160 
Table 3.5. Employment and Technology Spending......

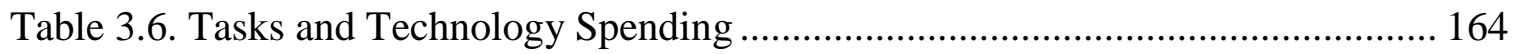

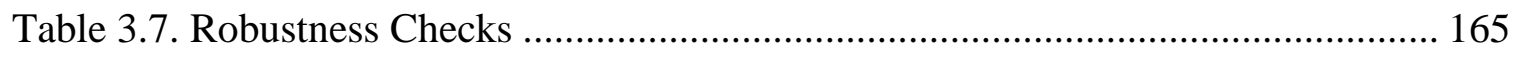




\section{LIST OF FIGURES}

FIGURE

PAGE

Figure 1.1: Operational Performance and Operational Efficiency .............................. 44

Figure 1.2: Firm Risk and Operational Efficiency.................................................. 45

Figure 1.3: Cumulative Return of Stock Portfolios Sorted by Standardized OER .......... 46

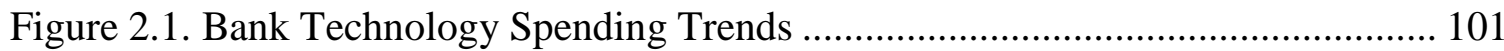

Figure 2.2. Performance and Technology Investment Over Time.............................. 102

Figure 2.3. Technology Investment Around Negative Performance Shocks................. 103

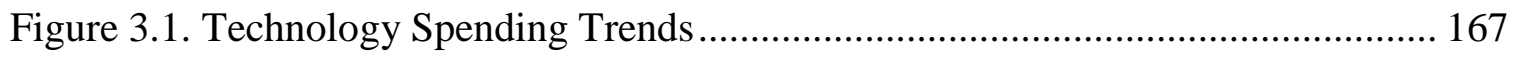

Figure 3.2. Employment and Technology Spending Over Time ............................... 168

Figure 3.3. Number of Employees and Technology Spending in the Cross-Sections .... 169

Figure 3.4. Staff Expense and Technology Spending in the Cross-Sections ................. 170 


\section{Chapter I: REIT Operational Efficiency: Performance, Risk, and Return}

\subsection{Introduction}

The literature focused on REITs is extensive. Most studies, however, focus on one of several broad areas including diversification benefits, acquisition strategies, differences in equity and mortgage investments, corporate governance and capital structure. ${ }^{1}$ Few studies investigate relations between revenues from real estate assets and the expenses needed to generate those revenues. Specifically, little work has been applied to (1) the appropriate classification of REIT revenues and expenses, such as gross rent, net rent, depreciation, amortization and tenant pass-throughs; and (2) exploring the performance and value implications associated with these relations. In the present research, measures of REIT operational efficiency similar to those found in the banking literature are introduced. These measures of efficiency, linking various types of operational expenses to revenues, are defined within a REIT context. The impact of these measures on REIT operational performance, risk and stock return is concurrently explored.

Efficiency in banking and financial institutions has been investigated in detail. The most common efficiency ratio found in the literature, and used by analysts and bank executives, is defined as a bank's non-interest expenses divided by revenue or net income (Bikker and Haaf, 2002; Bonin, Hasan, and Wachtel, 2005; Jacewitz and Kupiec, 2012).

In the Quarterly Banking Profile from the Federal Deposit Insurance Corporation (FDIC), efficiency is defined as "noninterest expense less amortization of intangible assets

\footnotetext{
${ }^{1}$ See Brounen and de Koning (2013) and Baker and Chinloy (2014) for more details.
} 
as a percent of net interest income plus noninterest income”. The FDIC further explains that "this ratio measures the proportion of net operational revenue that are absorbed by overhead expense, so that a lower value indicates greater efficiency."2 REITs are, in fact, as financial institutions in many ways. The National Association of Real Estate Investment Trusts (NAREIT) defines a REIT as “A company that owns or finances income-producing real estate. Modeled after mutual funds, REITs provide investors of all types of regular income streams, diversification and long-term capital appreciation. REITs typically pay out all of their taxable income as dividends to shareholders." ${ }^{3}$ A REIT is an intermediary that holds a portfolio of real estate assets and passes income and cash flows to its shareholders, and its value should be related to how efficient it is in providing this service.

While some REIT studies focus on technical efficiency, X-efficiency and economies of scale (Kuhle, Walther and Wurtzebach, 1986; Anderson, Lewis, and Springer, 2000; Anderson, Fok, Springer, and Webb, 2002; Devaney and Weber, 2005), this study employs an efficiency ratio that is based on the banking efficiency concept described above. The efficiency ratios are used to measure the amount of revenue REITs generate relative to operational expenses. Specifically, two REIT operational efficiency ratios are defined as: a) total expenses less real estate depreciation and amortization expense to total revenue and b) total expenses less real estate depreciation and amortization expense adjusted for property specific expenses to total revenue less expense

\footnotetext{
${ }^{2}$ https://www.fdic.gov/bank/analytical/qbp/glossary.html

${ }^{3}$ https://www.reit.com/investing/reit-basics/what-reit
} 
reimbursements. ${ }^{4}$ In the accounting and financial economics literature, similar ratios of operating expense divided by annual sales are used as an agency cost proxy because they serve as a measure of the effectiveness of management in controlling operations and direct agency costs (Ang, Cole and Lin, 2000).

Using a broad sample of U.S. equity REITs from the modern REIT era, the results show that REIT return on assets and REIT return on equity are strongly related to firm operating efficiency. The results suggest that more efficient REITs are associated with better operational performance. ${ }^{5}$ Further results show that REIT total risk and credit risk benefit from greater operational efficiency. It is also illustrated that REIT cross-sectional stock returns may be partially explained by operational efficiency. Besides, a portfolio consisting of more efficient REITs earns, on average, higher cumulative stock returns compared with a portfolio consisting of less efficient REITs. Overall, these findings illustrate the importance of REIT operational efficiency on performance, risk and return. ${ }^{6}$

${ }^{4}$ The measure is adjusted to reflect those costs that are directly associated with asset operations and management. The adjustment is made for expenses that are passed through to tenants. Not all property expenses are reimbursed so one also control for property type, which is the primary determinant of reimbursements.

${ }^{5}$ It is recognized that there still exists a potential endogeneity issue between operational efficiency and firm performance and there may be possible unobserved heterogeneity that determines the observed relation between operational efficiency and firm performance. As this is one of the first papers on the topic, it is likely that more research needs to be done to refine all potential conclusions.

${ }^{6}$ Theoretically, a reverse causality issue for REIT risk, especially stock return volatility, stock return and REIT operational efficiency should not exist. The empirical results that REIT operational efficiency has a negative (positive) relation with one period ahead firm risk (stock return) can provide reliable casual inference. It is not likely that the lower risk and/or higher return causes higher operational efficiency. 


\subsection{An Overview of Related Literature}

There is a rich banking literature on the efficiency of financial institutions. Most of the literature focuses on four types or categories of efficiency. The first type is scale efficiency. The idea is that financial institutions benefit from economies of scale. Hence, larger firms are more likely to have better performance (Berger, Hancock, and Humphrey, 1993; Berger, Hunter, and Timme, 1993). The second category is scope efficiency, whereby financial institutions benefit from lowering average costs by producing and selling a wide array of products (Zardkoohi and Kolari, 1994). The third efficiency measure is X-efficiency, which illustrates whether financial institutions are operating with an efficient mix of inputs, (Berger, Hunter, and Timme, 1993; Allen and Rai, 1996). Finally, the fourth and most common efficiency category is related to overall operational efficiency and is often measured with an efficiency ratio defined as non-interest expenses divided by revenues or net income (Bikker and Haaf, 2002; Bonin, Hasan, and Wachtel, 2005; Jacewitz and Kupiec, 2012). This efficiency measure is a straightforward indicator of overhead expenses relative to operational revenues. Financial institutions associated with lower ratios are more efficient.

Anderson, Lewis, and Springer (2000) provide a comprehensive review of the efficiency literature for real estate brokerage services and REITs at the advent of the modern REIT era. Allen and Sirmans (1987), Linneman (1997), Bers and Springer (1997) and Vogel (1997) show that REIT mergers and acquisitions are due in part to the existence of economies of scale. Similarly, Anderson, Fok, Zumpano, and Elder (1998) and Anderson, et al. (2002) analyze REIT scale economies and X-efficiencies using data envelopment analysis (DEA). They show that REITs are generally scale inefficient. In 
their narrow 1992-1996 sample period, REITs' overall efficiency scores measured between $44.1 \%$ and $60.5 \%$ (out of $100 \%$ ). They also show that large REITs are more efficient than small REITs and suggest that expansion may improve performance. Using a stochastic frontier methodology and Bayesian statistics to define REITs' efficient cost frontiers, Lewis, Springer, and Anderson (2003) find that REITs are almost 90\% efficient and show that REIT performance and efficiency are positively related.

There is, however, conflicting evidence concerning studies focused on economies of scale in REITs. For example, McIntosh, Liang, and Thompkins (1991) and McIntosh, Ott, and Liang (1995) provide evidence against the existence of scale economies. Similarly, Mueller (1998) and Ambrose, Ehrlich, Hughes, and Wachter (2000) show that smaller REITs are more profitable, indicating there may be an optimal REIT size based on their cash flows. More recently, Chung, Fung, and Hung (2010) show that institutional ownership can help reduce REITs' inefficiency. Other studies of the impact of institutional ownership on performance find few relations (Hartzell, Sun, and Titman, 2006; Bianco, Ghosh, and Sirmans, 2007; Bauer, Eichholtz, and Kok, 2010), with Hardin, Nagel, Roskelley, and Seagraves (2017) arguing that only a small set of investors will expend sufficient energy to monitor to improve operating performance. The ambiguity may also be related to the sample frame and the maturation of the REIT industry.

Bers and Springer (1998a, 1998b) use the ratio of different REIT costs, such as general and administrative (G\&A) expense, management fees, operating expenses, and interest expense, to total liabilities to examine scale economies. This measure, which is conceptually similar to the efficiency measures used in this paper, allowed them to show a negative cost elasticity associated with interest expense related to total liabilities. In a 
related paper, Bers and Springer (1997) assess differences in scale economies among a variety of REIT characteristics and find that internal or external management choice, capital structure, and property types are related to their scale economies.

The present investigation builds on this existing, older literature primarily focused on the pre-modern REIT era by introducing efficiency ratios adjusted for industry characteristics as found in the banking literature. The questions of interest are straightforward. Does REIT efficiency impact operational performance measures? And, are REITs rewarded for their efficiency?

\subsection{Data Sources and Summary of Statistics}

The firm characteristics for U.S. equity REITs for the modern REIT era (1995 2016) with annual frequency are collected from From SNL Financial. ${ }^{7}$ Each observation includes, total assets, total debt, total equity, total revenue ${ }^{8}$, total expenses, expense reimbursements $^{9}$, real estate depreciation and amortization, rental operational expense, share price, total dividends paid, common shares outstanding, implied market capitalization, earnings before interest, tax, depreciation and amortization, funds from

\footnotetext{
7 The sample period starts in 1995 because the property level data are used to calculate geographic diversification and property type diversification are only available from 1995. For robustness, an extended sample with a longer period shows quantitatively similar empirical results, while not controlling for diversification. Also, only publicly traded REITs is addressed as Seguin (2016), Soyeh and Wiley (2017) and others argue that these firms are sufficiently different to warrant segmentation.

${ }^{8}$ All revenue including nonrecurring. Revenue is net of interest expenses for banks, thrifts, lenders, FHLBs, investment companies, asset managers and broker-dealers, as defined by SNL.

${ }^{9}$ Expenses reimbursed from tenants for common area maintenance and improvements, including operating expenses such as real estate taxes, insurance, and utilities, as defined by SNL.
} 
operations (FFO), IPO date, the year the REIT was established, the metropolitan statistical area (MSA) of properties, and real estate property type. ${ }^{10}$ Stock return data from the Center for Research in Security Prices (CRSP) and market factors and risk-free rate data from Kenneth French's website are also obtained. ${ }^{11}$

REIT operational efficiency ratio (OER) in general terms is defined as total operational expenses divided by revenue. Hence, the higher (lower) the efficiency ratio, the less (more) efficient the REIT. More specifically, two variations of the general REIT operational efficiency ratio are defined as: a) the ratio of non-real-estate-depreciation-andamortization expense, defined as total expenses minus real estate depreciation and amortization, to total revenue, and b) the ratio of non-real-estate-depreciation-andamortization expense adjusted for property expenses to total revenue less expense reimbursements. These two variations account for real estate depreciation and amortization and property operational expense reimbursements to better reflect the more controllable cash flow related expenses associated with each REIT.

The cost of holding and maintaining real properties varies across property type as does lease structure. Hence, operational expense ratios likely vary due to the type of properties owned. It is needed to employ measures that adjust for operational efficiency differences for REITs that are associated with real estate property types. These

\footnotetext{
${ }^{10}$ When REIT accounting information is not available in one period, but is available for the pervious and subsequent periods, it is replaced by the estimation calculated from the characteristics in previous and subsequent periods using the formula: : Value $e_{i, t}^{x}=\left(\right.$ Value $_{i, t+1}^{x}+$ Value $\left._{i, t-1}^{x}\right) / 2$. Where Value $_{i, t}^{x}$ is the value of $x$ (TA, TE, etc.) of REIT $i$ in year $t$.

${ }^{11}$ Kenneth R. French's Data Library: http://mba.tuck.dartmouth.edu/pages/faculty/ken.french/data_library.html.
} 
standardized operational efficiency measures (OERI and OER2) are defined as the operational efficiency ratio of each REIT divided by the mean of the operational efficiency ratios of all REITs that specialize in the same real estate property type in that year.

REIT operational performance is measured as return on assets (ROA), which is defined as funds from operations divided by total assets in the previous period, and return on equity (ROE), which is defined as funds from operations divided by total equity in the previous period. ${ }^{12}$ REIT total risk is measured by the standard deviation of the annualized stock return and can also be referred to as stock return volatility. REIT credit risk is proxied by the EBITDA-to-Debt ratio. The stock return for a REIT is defined as the sum of the share price change and dividends divided by share price in the previous period. Other variables used in this study include firm size, which is defined as the logarithm of implied market capitalization; leverage ratio, which is defined as the ratio of total book assets to total book equity, following Adrian and Shin (2010); firm age, which is defined as logarithm of one plus firms' years since IPO ${ }^{13}$; geographic diversification, which is defined as the negative of the Herfindahl Index of each REIT, calculated using assets invested in different MSA locations, based on book values, as in Hartzell, Sun, and Titman (2014); property type diversification, which is defined as the negative of the Herfindahl Index of each REIT, calculated using assets invested in different real estate property types, based on book values, as in Hartzell, Sun, and Titman (2014); and firm classification and whether the firm is in the S\&P Index, which is a binary variable that takes a value of 1 when a REIT

\footnotetext{
12 These are common performance metrics for REITs.

${ }^{13}$ When the IPO date is not available, the year a REIT status is established is used instead.
} 
is in the S\&P index. The variables used in this paper along with their definition are displayed in the Appendix.

Because the regression specification includes lagged variables, firms with fewer than two consecutive years of stock price and operational efficiency information are excluded. Variables have been winsorized at the $1 \%$ and $99 \%$ tails of the distributions. The final sample used in the analysis consists of 317 REITs.

Table 1.1 provides summary statistics for the REITs included in the sample including operational performance, risk, stock return, and operational efficiency measures. Over the full sample period (1995 - 2016), the average REIT market capitalization has a mean of $\$ 2.3$ billion and a median of $\$ 0.9$ billion. Total REIT revenue per year has a mean of $\$ 0.4$ billion and a median of $\$ 0.2$ billion. Return on assets (ROA) has an average of $6.04 \%$ and a median of $6.05 \%$, while return on equity (ROE) has an average of $16.39 \%$ and a median of $14.31 \%$. The mean and median of annual stock return volatility are 0.30 and 0.23, and the mean and median for the EBITDA-to-Debt ratio are 0.19 and 0.16. The average annual stock return during the examined period is $12.99 \%$, with a median of 12.97\%. In terms of the operational efficiency ratios, the mean and median of the standardized operational efficiency ratio type one (OERI) are 0.99 and 0.96 , and mean and median of the standardized operational efficiency ratio type two (OER2) are 0.99 and 0.91 .

\subsection{Research Methodologies}

The analysis begins with an evaluation of whether a REIT's operational performance is associated with its operational efficiency ratios. Specifically, the regression REIT return on assets on each of the measures of operational efficiency while controlling 
for REITs characteristics is adopted. An ordinary least squares (OLS) model with heteroscedasticity-robust standard errors that are clustered at the firm level and with the property type and year fixed effects (or with firm and year fixed effects) is used, as follows.

$$
\begin{aligned}
\text { ROA }_{i, t}=\beta_{0}+ & \beta_{1} \text { Size }_{i, t-1}+\beta_{2} \text { Leverage }_{i, t-1}+\beta_{3} \text { Firm Age }_{i, t-1} \\
& +\beta_{4} \text { Geographic Diversification }_{i, t-1} \\
& +\beta_{5} \text { Property Type Diversification }_{i, t-1} \\
& +\beta_{6} \text { S\&P Index } \text { I }_{i, t-1}+\beta_{7} \text { OE }_{i, t-1}+\varepsilon_{i, t}
\end{aligned}
$$

Where $\mathrm{ROA}_{\mathrm{i}, \mathrm{t}}$ is the funds from operations divided by lagged total assets of REIT $i$ at year $t \mathrm{~m}$ and the other variables included in equation (1) are as defined earlier in the text. Additionally, the multivariate regression from equation (1-1) using a non-parametric analysis approach by sorting REITs into quintiles based on their standardized operational efficiency ratios in each year is applied. The spreads of the mean and median of the ROA from the extreme quintiles, along with their associated two-sample $t$ test and Wilcoxon rank-sum test values are aslo reported.

The use of lagged property portfolio characteristics as explanatory variables provides adjustment to reflect the beginning annual portfolios held by a REIT. Performance should be more reflective of the characteristics of the REIT properties at the start of the year than at the end of the year. This can be important in the REIT industry where holding periods are long-term and where the industry has expanded dramatically over the last two decades. Cash flow generation and expenses follow in large measure the properties held at the beginning of each period in combination with changes in the portfolio during the interim period versus the ending period composition of the portfolio. It is needed to adjust 
other variables for comparability and to mitigate potential issues related to endogeneity. The general concept is to create the basic firm and managerial characteristics for the firm before the period of assessment.

For a visual illustration, figures that plot the measures of return on assets versus each of the standardized operational efficiency ratios for the previous year are provided. The slope, $t$-statistics, $p$-value and adjusted $R$-squared from the univariate regression associated with each figure are reported on the top of each figure.

Return on equity is another profitability ratio that measures the ability of a firm to generate profits. It can be argued from the shareholder's perspective that return on equity is the best indicator of firm performance (Elayan, Meyer, and Li, 2009) as an investment. Hence, whether REIT return on equity (ROE) is associated with the two measures of operational efficiency is explored.

$$
\begin{aligned}
\text { ROE }_{i, t}=\beta_{0}+ & \beta_{1} \text { Size }_{i, t-1}+\beta_{2} \text { Leverage }_{i, t-1}+\beta_{3} \text { Firm Age }_{i, t-1} \\
& +\beta_{4} \text { Geographic Diversification }_{i, t-1} \\
& +\beta_{5} \text { Property Type Diversification }_{i, t-1} \\
& +\beta_{6} \text { S\&P Index }_{i, t-1}+\beta_{7} \text { OER }_{i, t-1}+\varepsilon_{i, t}
\end{aligned}
$$

where $R O E_{i, t}$ is the funds from operations, respectively, divided by lagged total equity of REIT $i$ at year $t$, and other variables are as defined previously. The multivariate regression from equation (1-2) using a non-parametric analysis approach is applied and figures which plots the measures of return on equity versus each of the standardized operational efficiency ratios for the previous year are created. 
A similar approach is used to examine the relations between REIT total risk, credit risk, and operational efficiency. Total risk is measured as annualized stock return volatility, and credit risk is measured as the EBITDA-to-Debt ratio, which is an indicator of a REIT's ability to satisfy its debt payment obligations.

$$
\begin{aligned}
\text { Risk }_{i, t}=\beta_{0}+ & \beta_{1} \text { Size }_{i, t-1}+\beta_{2} \text { Leverage }_{i, t-1} \\
& +\beta_{3} \text { Firm Age }_{i, t-1} \\
& +\beta_{4} \text { Geographic Diversification }_{i, t-1} \\
& +\beta_{5} \text { Property Type Diversification }_{i, t-1} \\
& +\beta_{6} \text { S\&P Index }_{i, t-1}+\beta_{7} \text { OER }_{i, t-1}+\varepsilon_{i, t}
\end{aligned}
$$

Where Risk $_{i, t}$ is the annualized stock return volatility and EBITDA divided by total debt, respectively, of REIT $i$ at year $t$, and the other variables are as previously defined. Once again, the multivariate regression from equation (1-3) using a non-parametric analysis approach is applied and figures which plots the measures of return on equity versus each of the standardized operational efficiency ratios for the previous year are created.

Finally, whether REIT operational efficiency ratios help explain the cross-sectional stock return of REITs is examined. Specifically, the regression of annual excess REIT stock return using the Fama French (1993) three-factor model, the Carhart (1997) four-factor model and the Fama French (2015) five-factor model while including the REIT operational efficiency variable are adopted as follows: ${ }^{14}$

\footnotetext{
${ }^{14}$ Similar models can be found in Baker and Wurgler (2006) and Giacomini, Ling, and Naranjo (2016), among others.
} 


$$
\begin{gathered}
\operatorname{Return}_{i, t}=\alpha_{0}+\alpha_{1} r m r f_{t}+\alpha_{2} s m b_{t}+\alpha_{3} h m l_{t}+\beta_{1} O E R_{i, t}+\varepsilon_{i, t} \\
\operatorname{Return}_{i, t}=\alpha_{0}+\alpha_{1} r m r f_{t}+\alpha_{2} s m b_{t}+\alpha_{3} h m l_{t}+\alpha_{4} m o m_{t} \\
+\beta_{1} O E R_{i, t}+\varepsilon_{i, t} \\
\operatorname{Return}_{i, t}= \\
\alpha_{0}+\alpha_{1} r m r f_{t}+\alpha_{2} s m b_{t}+\alpha_{3} h m l_{t}+\alpha_{4} r m w_{t}+\alpha_{5} \operatorname{cma}_{t} \\
+\beta_{1} O E R_{i, t}+\varepsilon_{i, t}
\end{gathered}
$$

Where Return $_{i, t}$ is the annual stock return of REIT $i$ minus the risk-free rate at year $t$; $r m r f_{t}$ is the value-weighted market return minus the risk-free rate at year $t ; s m b_{t}$ (Small minus Big), $h m l_{t}$ (High minus Low), mom $_{t}$ (Momentum), $r m w_{t}$ (Profitability) and $c m a_{t}$ (Investment) are the year $t$ return to zero investment factor-mimicking portfolios designed to capture size, book-to-market, momentum, profitability and investment effects, respectively. $\beta_{1}$ is the coefficient of interest in this regression, as it captures the relations between REIT stock return and the operational efficiency ratios after controlling for market risk.

Alternatively, a similar approach to examine the relations between REIT stock return and REIT operational efficiency is adopted.

$$
\begin{aligned}
& \text { Residual Return }_{i, t} \\
& =\beta_{0}+\beta_{1} \text { Size }_{i, t-1}+\beta_{2} \text { Leverage }_{i, t-1} \\
& +\beta_{3} \text { Firm Age } e_{i, t-1} \\
& +\beta_{4} \text { Geographic Diversification }_{i, t-1} \\
& +\beta_{5} \text { Property Type Diversification }{ }_{i, t-1} \\
& +\beta_{6} S \& P \text { Index }_{i, t-1}+\beta_{7} O E R_{i, t-1}+\varepsilon_{i, t}
\end{aligned}
$$


Where Residual Return $n_{i, t}$ is residual excess stock return, which is obtained from the Fama-French (2015) five-factor model, of REIT $i$ at year $t$, and the other variables are as previously defined.

To further evaluate whether REIT operational efficiency ratios have a long-term effect on stock returns, portfolios by sorting the standardized operational efficiency ratios (OER1 and OER2) of each REIT in the previous year are constructed. Specifically, REITs are divided based on the median (or 30 and 70 percentiles) of their OER 1 and $O E R 2$, respectively, and REITs with above or below median (or 70 or 30 percentiles) OER 1 and $O E R 2$ are placed in the low or high efficiency portfolios, respectively. These portfolios are rebalanced each year. Then, the one- to four- year cumulative return of these operational efficiency based portfolios are compared.

\subsection{Empirical Results}

\subsubsection{Operational Performance and Operational Efficiency}

As described in the methodology section, relations between the REIT operational efficiency ratios and REIT operational performance measured by return on assets (ROA) and return on equity (ROE) are explored. The results from equation (1-1) are reported in Panel A of Table 2. Overall, the results provide evidence that more efficient REITs have, on average, higher returns on assets, even after controlling for size, financing, management structure, diversification and growth strategy.

In columns (1) and (2), the coefficients of the previous year OERI and OER2 variables are negative with statistical significance at the $1 \%$ level $(-5.27$ and -3.24 , respectively) in a property type and year fixed effect model. These results suggest that more 
efficient REITs (lower efficiency ratio) generate higher ROAs. The results presented with a firm and year fixed effect model as in columns (3) and (4) are very similar to the results presented in columns (1) and (2) and display statistical significance at the $1 \%$ level. The estimated coefficients of -2.97 and -1.76 for the previous year $O E R 1$ and $O E R 2$ variables, respectively, suggest a positive relation between REIT efficiency and ROA. ${ }^{15}$

In addition to the coefficients of interest, REITs with higher market capitalization, lower leverage, and less geographic diversification are associated with higher ROA are also shown. This is in line with expectations and is consistent with the literature. Larger REITs usually perform better (Berger, Hancock, and Humphrey, 1993; Berger, Hunter, and Timme, 1993; Ambrose, Highfield, and Linneman, 2005) and the negative relationship between firm performance and leverage is widely found in the finance literature (e.g. Titman and Wessels, 1988; Rajan and Zingales, 1995; Fama and French, 2002). It is also well-known that there exists a diversification discount on firm performance or valuation, as in, for example, Lang and Stulz (1994), Capozza and Seguin (1999), Cronqvist, Högfeldt, and Nilsson (2001), Campa and Kedia (2002), Danielsen and Harrison (2007), Ro and Ziobrowski (2011), Hartzell, Sun and Titman (2014), and Ling, Ooi, and Xu (2016).

It is worth noting that achieving a higher relative level of return on assets is difficult to do in a capital-intensive business such as equity REITs. This further highlights the importance of REITs operational efficiency on operational performance.

\footnotetext{
${ }^{15}$ It is recognized that REIT operational efficiency may also be an endogenous outcome of managerial decisions and other factors. For instance, ownership structure, corporate governance, investments in a growing market just by chance.
} 
The positive relation between operating efficiency and operating performance also shows in the univariate regression models. Panel A of Figure 1-1 plots ROA versus each of the previous year standardized operational efficiency ratios (OERI and $O E R 2)$. The negative slope is visually clear in each of the plots.

Panel B of Table 1-2 presents the results from a quintile analysis approach that compares REIT mean and median ROA sorted by their previous year standardized operational efficiency ratios (OERI and $O E R 2)$. The results show that the mean and median ROA of REITs sorted by previous year standardized operational efficiency ratios decrease monotonically from the first quintile (highest operational efficiency) to the fifth quintile (lowest operational efficiency) in both cases. The spreads of the mean (median) of ROA between the two extreme quintiles is $4.74 \%(4.27 \%)$ and $4.29 \%$ (3.73\%), respectively. Each of these differences is statistically significant at the $1 \%$ level using the $t$-statistic from the two-sample $t$-test or the $z$-statistics from the two-sample Wilcoxon rank-sum test. The results from the non-parametric analysis support the multivariate regression results and clearly show, not only positive relations between return on assets and operational efficiency, but that the relation is monotonic and continuous.

The results from equation (1-2) are reported in Panel A of Table 1-3. Overall, the results presented in this panel are very similar to the results reported in Panel A of Table $1-2$, where the relationship between return on assets and operational efficiency is examined. The coefficients of the operating efficiency measures are negative and statistically significant in all four specifications. These results support the results from the previous table and suggest that REIT operating efficiency is positively related to return on equity. All else equal, if a REIT can decrease its OERI by $1 \%$, it would realize an average ROE 
increase of 11.87 basis points (column (1)). Also, similar to the results from Panel A of Table 1-2, there is evidence for positive relations between return on equity and leverage. Consistent results can also be found in Panel A of Figure 1-2, which plots ROE versus each of the previous year $O E R 1$ and $O E R 2$ measures. The negative slope (positive relation between operational efficiency and return on equity) is visually clear.

Like Panel B of Table 1-2, Panel B of Table 1-3 presents the results from a quintile analysis. Again, the results of this panel are like the results presented in Table 1-2. The spreads of the mean and median of ROE between the first quintile (highest operational efficiency) to the fifth quintile (lowest operational efficiency) of REITs sorted by previous year standardized operational efficiency ratios are statistically significant at the $1 \%$ level.

Collectively, the results provide strong evidence that REIT operational performance is positively related to the efficient management of the firm measured by the previous year's operational efficiency. On average, more efficient REITs (lower operational efficiency ratios) generate higher returns on assets and returns on equity.

\subsubsection{Firm Risk and Operational Efficiency}

The results presented in this subsection shed light on the extent to which a REIT's risk is associated with its operational efficiency ratios. As mentioned earlier, REIT total risk is measured as annualized stock return volatility, and REIT credit risk is measured as the EBITDA-to-Debt ratio. Stock return volatility plays an essential role in the finance literature, including asset pricing, cost of capital, risk management, and asset allocation. There is ample evidence that higher volatility is associated with higher expected returns. The EBITDA-to-Debt ratio measures the ability of a firm to withstand a negative shock to 
its profitability without defaulting on its debt obligations. This measure is especially important for REITs given that the real estate sector is more levered than most other industry sectors (Morri and Beretta, 2008). Moreover, unlike other firms, the ability of REITs to fund investments via internally generated cash flows is limited due to their mandatory distribution requirement of at least $90 \%$ of earnings to shareholders. As a result, large REIT investments are more likely to be funded by the use of debt, at least in the short run, or an increase in share count.

The results from Equation (1-3) when stock return volatility is the dependent variable are reported in Panel A of Table 1-4. The positive coefficients, 0.078 and 0.045, respectively, of previous year OERI and OER2 in columns (1) and (2), with statistical significance at $1 \%$, indicate that REITs with higher efficiency ratios (lower operating efficiency) have, on average, higher stock return volatility. The results presented with a firm and year fixed effect model as in columns (3) and (4) are very similar to the results presented in columns (1) and (2). The results imply that more efficient REITs (lower efficiency ratio) are exposed to less total return risk.

Regarding the other factors impacting firm-level risk, the results are generally in line with the existing REIT literature (e.g., Tom and Austin, 1996; Allen, Madura, and Springer, 2000; Tien and Sze, 2003). REITs with higher market capitalization are associated with lower total risk. Consistent with the REIT literature and what has been shown in banking (e.g., Demsetz and Strahan, 1997), size-related diversification leads to reductions in firm-specific risk (e.g., Norman, Sirmans, and Benjamin, 1995; Gyourko and Nelling,1996; Tom and Austin, 1996). Younger REITs appear to be less risky, which warrants additional research and may be related to the newness of the REIT industry and 
conversions of private portfolios to publicly traded vehicles. Variables addressing more geographic diversification and inclusion in $\mathrm{S} \& \mathrm{P}$ indices, on average, have higher total risk in the property type and year fixed effect model as in columns (1) and (2). However, those variables are not statistically significant in the firm and year fixed effect model as in columns (3) and (4).

Like Figure 1-1, Panel A of Figure 1-2 plots the univariate results of stock return volatility versus previous year $O E R I$ and $O E R 2$. The slope, $t$-statistics, $p$-value and adjusted $R$-squared are reported on the top of each figure. The results are consistent with the findings reported using multivariate regression.

Panel B of Table 1-4 presents the quintile analysis results. These results support the results presented in the previous panel. The means and medians of stock return volatility are monotonically increasing from the first quintile (highest operational efficiency) to the fifth quintile (lowest operational efficiency) of REITs sorted by previous year OERI and OER2. The mean (median) difference between these extreme quintiles are $0.09(0.05)$ and 0.08 (0.04) for $O E R 1$ and $O E R 2$, respectively, and associated with high statistical significance.

When the EBITDA-to-Debt ratio is the dependent variable in Equation (1-3), the results are reported in Panel A of Table 1-5. The estimated coefficients of $O E R I$ and $O E R 2$ in columns (1) to (2) for EBITDA-to-Debt ratio are both negative (-0.13, and -0.09 , respectively) and statistically significant at the $1 \%$ level. Quantitively similar results with a firm and year fixed effect model can be found in columns (3) and (4). Together, the results imply that more efficient REITs (lower efficiency ratio) are associated with lower debt 
levels relative to their cash flow. Aside from the coefficients of interest, the results also show that REITs with lower debt are associated with less credit risk, as expected.

Panel B of Table 1-5 presents the quintile analysis results. The means and medians of EBITDA-to-Debt ratio are monotonically decreasing from the first to the fifth quintile. The spreads of the mean (median) between the two extreme quintiles are $0.11(0.07)$ and 0.10 (0.06) for $O E R 1$ and $O E R 2$, respectively, and are significant at $1 \%$ level in both the two-sample $t$-test and Wilcoxon rank-sum test.

\subsubsection{Stock Return and Operational Efficiency}

As a final step, after examining the relationship between operational efficiency and operational performance and risk, whether REITs' operational efficiency is related to their stock return is investigated.

Table 1-6 presents the OLS regression coefficient estimates of the Fama French (1993) three-factor model, the Carhart (1997) four-factor model and the Fama French (2015) five-factor model along with a REIT operational efficiency variable, as in Equation (4). REIT stock return net of the risk-free rate is the dependent variable in these regressions. In each of the four specifications, the operational efficiency ratio used is found to be negative and statistically significant at the $1 \%$ level. More specifically, the estimated coefficients associated with OERl in columns (1) to (3) are $-9.74,-9.67$ and -9.62 , respectively, while those with $O E R 2$ in columns (4) to (6) are $-6.33,-6.30$ and -6.23 , respectively.

The regression analysis indicates that a portion of REIT expected returns that cannot be explained by the common market factors is associated with REIT operational 
efficiency. As the efficiency ratios proposed in this paper measure the amount of revenue REITs generate relative to their operational expenses, such information should be unique for each REIT and not related to market-wide shocks from either real estate or capital markets.

Also, the residual excess stock return obtained from the Fama-French (2015) fivefactor model is used to explore relations between REIT stock return and REIT operational efficiency ratios. The results from equation (1-5) are reported in Table 1-7. The results provide evidence that more efficient REITs have, on average, higher stock returns which could not be explained by the common market factors, even after controlling for size, financing, management, diversification and growth strategy. ${ }^{16}$ More specifically, the estimated coefficients associated with OERI in columns (1) and (3) are -10.63 and -7.11, respectively, while those with $O E R 2$ in columns (2) and (4) are -6.45 and -3.57 , respectively. The result suggests that REITs that exhibit higher operational efficiency are associated with higher risk-adjusted stock returns, as expected. REITs with operational effectiveness and efficiency generate better results for given portfolios of real estate, which is reflected in stock performance. REIT operational efficiency captures the relative ability to generate cash flows, which is concomitantly related to the management of the firm and assets related to managerial structure, employee retention, and human capital.

Finally, to determine whether cumulative stock returns are different between high and low efficiency REITs, portfolios are constructed by sorting REITs based on their

\footnotetext{
${ }^{16}$ For robustness, residual stock return is also obtained via the Fama French (1993) three-factor model and the Carhart (1997) four-factor model. Quantitatively similar results are found. For brevity, these results are not reported, but are available upon request.
} 
previous year standardized operational efficiency ratio (OER1 and OER2) and then the cumulative return differentials for periods of one to four years after portfolio formation are examined. The results of this analysis are illustrated in Figure 1-3.

A glance at Figure 1-3 reveals that, in the medium term, portfolios that consist of low efficiency REITs materially underperform portfolios that consist of high efficiency REITs. Specifically, the four-year cumulative return differential between the portfolio consisting of the bottom $30 \%$ of OER1 and the portfolio consisting of the top 30\% of OER1 is about 8\%, as shown in Panel A. Similarly, the four-year cumulative return differential between the portfolio consisting of the bottom $30 \%$ of $O E R 2$ and the portfolio consisting of the top $30 \%$ of $O E R 2$ is also as large as $8 \%$, as showed in Panel B. These results are consistent with the findings presented in Table 1-5. Portfolios taken from the more efficient REITs outperform portfolios derived from the less efficient REITs

\subsection{Robustness Checks}

Since the analysis uses lagged variables in explaining the relationship between REIT operational performance and operational efficiency in Section 5.1, a correlation table with current period and previous period variables is provided. The correlation table indicates whether the variables of interest persistent. Panel A of Table 1-8 shows the results on the pair-wise correlation of the regression variables. The operational performance of REITs is strongly correlated with their previous-year operational performance. The correlation of ROA at year $t$ and year $t-1$ is 0.68 , while the correlation of ROE at year $t$ and year $t-1$ is 0.53 . There exists high persistence in operational efficiency measures. The 
correlation of current- and previous-year $O E R I$ and $O E R 2$ is 0.63 and 0.64 , respectively. More importantly, the correlation of ROA and ROE with current year OERI (OER2) is $0.66(-0.61)$ and $-0.25(-0.25)$, respectively, and previous year OER1 (OER2) is -0.43 ($0.40)$ and $-0.09(-0.10)$, respectively. As a higher operational efficiency ratio (OER) indicates a less efficient REIT, this result further suggests the existence of a positive relationship between REIT operational efficiency and operational performance.

Besides the possibility that lagged dependent variables may cause the coefficients for explanatory variables to be biased downward, if residual autocorrelation exists, the correlation results on current period and previous period variables also motivate us to examine other relationships further. Specifically, the relationship in cross-section by regressing REIT performance (ROA and ROE) on their current year standardized operational efficiency ratios ( $O E R I$ and $O E R 2$, respectively), while controlling for current year firm size, financing, management, diversification, and growth strategy, as in equation (1-1). The results of this analysis are reported in Panel B of Table 1-8. The estimated parameters for $O E R 1$ and $O E R 2$ are quantitatively and qualitatively greater than those reported in Tables 1-2 and 1-3, where the lagged variables are used. In a property type and year fixed effect model, the estimated coefficients of current year OERI are -7.75 when the dependent variable is ROA and -18.61 when the dependent variable is ROE. The estimated coefficients of current year $O E R 2$ are -5.03 when the dependent variable is ROA and -12.66 when the dependent variable is ROE, with statistical significance at the $1 \%$ level, as in Columns (1), (2), (5) and (6). The estimated coefficients of current year operational efficiency measures are quantitatively and qualitatively similar in a firm and 
year fixed effect models as in Columns (3), (4), (7) and (8). These consistent results provide a further evaluation of the sensitivity of the estimated parameters and further confirming a positive relation between REIT efficiency and performance.

\subsection{Conclusions}

The paper defines REIT operational efficiency and examines the extent to which REIT operational efficiency is related to operational performance, total risk, credit risk, and stock return. Using a sample of U.S. equity REITs during the modern REIT era (1995 - 2016), results show that more efficient REITs are associated with higher operational performance measured by return on assets and return on equity. Similarly, the results of the analysis show that more efficient REITs post lower stock return volatility and are associated with lower credit risk, measured by their EBITDA-to-Debt ratio. Furthermore, there is evidence that higher efficiency REITs outperform, on average, lower efficiency REITs in terms of risk-adjusted cross-sectional stock return as well as in terms of cumulative stock return in the medium term.

Collectively, the findings illustrate the importance of correctly measuring and accounting for REIT operational efficiency. This work has potential implications for REIT management, shareholder relations, REIT valuation, and portfolio allocation decisions. Moreover, a trading strategy that uses operational efficiency may yield higher returns. The research opens the door for more research on REIT operational efficiency to include institutional ownership and governance factors that might impact operational efficiency. Further research that examines in detail the importance of the components of REIT revenue 
and expenses concurrent with management and ownership structure will likely yield considerable insights.

References

Adrian, T., \& Shin, H. S. (2010). Liquidity and leverage. Journal of Financial Intermediation, 19(3), 418-437.

Allen, P. R., \& Sirmans, C. F. (1987). An analysis of gains to acquiring firm's shareholders: the special case of REITs. Journal of Financial Economics, 18(1), 175-184.

Allen, L., \& Rai, A. (1996). Operational efficiency in banking: an international comparison. Journal of Banking and Finance, 20(4), 655-672.

Allen, M. T., Madura, J., \& Springer, T. M. (2000). REIT characteristics and the sensitivity of REIT returns. The Journal of Real Estate Finance and Economics, 21(2), 141-152

Ambrose, B. W., Ehrlich, S. R., Hughes, W. T., \& Wachter, S. M. (2000). REIT economies of scale: fact or fiction?. The Journal of Real Estate Finance and Economics, 20(2), 211-224.

Anderson, R., Fok, R., Zumpano, L., \& Elder, H. (1998). Measuring the efficiency of residential real estate brokerage firms. Journal of Real Estate Research, 16(2), 139-158.

Anderson, R. I., Fok, R., Springer, T., \& Webb, J. (2002). Technical efficiency and economies of scale: a non-parametric analysis of REIT operating efficiency. European Journal of Operational Research, 139(3), 598-612.

Anderson, R., Lewis, D., \& Springer, T. (2000). Operating efficiencies in real estate: A critical review of the literature. Journal of Real Estate Literature, 8(1), 1-18.

Anderson, R., \& Reeb, D. (2003). Founding-family ownership and firm performance: evidence from the S\&P 500. The Journal of Finance, 58(3), 1301-1328

Ang, J. S., Cole, R. A., \& Lin, J. W. (2000). Agency costs and ownership structure. The Journal of Finance, 55(1), 81-106.

Baker, H. K., \& Chinloy, P. (Eds.). (2014). Public real estate markets and investments. Oxford University Press, USA. 
Baker, M., \& Wurgler, J. (2006). Investor sentiment and the cross-section of stock return. The Journal of Finance, 61(4), 1645-1680.

Banz, R. W. (1981). The relationship between return and market value of common stocks. Journal of Financial Economics, 9(1), 3-18.

Bauer, R., Eichholtz, P., \& Kok, N. (2010). Corporate governance and performance: The REIT effect. Real Estate Economics, 38(1), 1-29.

Berger, A. N., Hancock, D., \& Humphrey, D. B. (1993). Bank efficiency derived from the profit function. Journal of Banking and Finance, 17(2), 317-347.

Berger, A. N., Hunter, W. C., \& Timme, S. G. (1993). The efficiency of financial institutions: a review and preview of research past, present and future. Journal of Banking and Finance, 17(2), 221-249.

Bers, M., \& Springer, T. (1997). Economies-of-scale for real estate investment trusts. Journal of Real Estate Research, 14(3), 275-290.

Bers, M., \& Springer, T. M. (1998a). Sources of scale economies for REITs. Real Estate Finance, 14(4), 47-56.

Bers, M., \& Springer, T. M. (1998b). Differences in scale economies among real estate investment trusts: more evidence. Real Estate Finance, 15(1), 37-44.

Bianco, C., Ghosh, C., \& Sirmans, C. F. (2007). Corporate governance and firm performance - evidence from REITs. Journal of Portfolio Management, 33(5), 175-191.

Bikker, J. A., \& Haaf, K. (2002). Competition, concentration and their relationship: An empirical analysis of the banking industry. Journal of Banking and Finance, 26(11), 2191-2214.

Bonin, J. P., Hasan, I., \& Wachtel, P. (2005). Bank performance, efficiency and ownership in transition countries. Journal of Banking and Finance, 29(1), 31-53.

Brounen, D., \& de Koning, S. (2013). 50 years of real estate investment trusts: An international examination of the rise and performance of REITs. Journal of Real Estate Literature, 20(2), 197-223.

Campa, J. M., \& Kedia, S. (2002). Explaining the diversification discount. The Journal of Finance, 57(4), 1731-1762.

Capozza, D. R., \& Seguin, P. J. (1999). Focus, transparency and value: the REIT evidence. Real Estate Economics, 27(4), 587-619. 
Carhart, M. M. (1997). On persistence in mutual fund performance. The Journal of Finance, 52(1), 57-82.

Chaudhry, M., Maheshwari, S., \& Webb, J. (2004). REITs and idiosyncratic risk. Journal of Real Estate Research, 26(2), 207-222.

Chung, R., Fung, S., \& Hung, S. Y. K. (2012). Institutional investors and firm efficiency of real estate investment trusts. The Journal of Real Estate Finance and Economics, 45(1), 171-211.

Cronqvist, H., Högfeldt, P., \& Nilsson, M. (2001). Why agency costs explain diversification discounts. Real Estate Economics, 29(1), 85-126.

Danielsen, B., \& Harrison, D. (2007). The impact of property type diversification on REIT liquidity. Journal of Real Estate Portfolio Management, 13(4), 329-344.

Demsetz, R. S., \& Strahan, P. E. (1997). Diversification, size, and risk at bank holding companies. Journal of Money, Credit, and Banking, 300-313.

Devaney, M., \& Weber, W. L. (2005). Efficiency, scale economies, and the risk/return performance of real estate investment trusts. The Journal of Real Estate Finance and Economics, 31(3), 301-317.

Elayan, F., Meyer, T., \& Li, J. (2006). Evidence from tax-exempt firms on motives for participating in sale-leaseback agreements. Journal of Real Estate Research, 28(4), 381-410.

Fama, E. F., \& French, K. R. (2002). Testing trade-off and pecking order predictions about dividends and debt. The Review of Financial Studies, 15(1), 1-33.

Fama, E. F., \& French, K. R. (1993). Common risk factors in the returns on stocks and bonds. Journal of Financial Economics, 33(1), 3-56.

Fama, E. F., \& French, K. R. (2015). A five-factor asset pricing model. Journal of Financial Economics, 116(1), 1-22.

Feng, Z., Ghosh, C., He, F., \& Sirmans, C. F. (2010). Institutional ownership and REIT CEO compensation. The Journal of Real Estate Finance and Economics, 40(), 446-479

Giacomini, E., Ling, D. C., \& Naranjo, A. (2017). REIT leverage and return performance: Keep your eye on the target. Real Estate Economics, forthcoming.

Hardin III, W. G., Nagel, G., Roskelley, K. D., \& Seagraves, P. A. (2017). Motivated institutional monitoring and firm performance. Journal of Real Estate Research, 39:3, 401-439. 
Hartzell, J. C., Sun, L., \& Titman, S. (2014). Institutional investors as monitors of corporate diversification decisions: Evidence from real estate investment trusts. Journal of Corporate Finance, 25, 61-72.

Jacewitz, S., \& Kupiec, P. (2012). Community bank efficiency and economies of scale. FDIC Special Study, December.

Kuhle, J., Walther, C., \& Wurtzebach, C. (1986). The financial performance of real estate investment trusts. Journal of Real Estate Research, 1(1), 67-75.

Lang, L. H., \& Stulz, R. M. (1994). Tobin's q, corporate diversification, and firm performance. Journal of Political Economy, 102(6), 1248-1280.

Lewis, D., Springer, T. M., \& Anderson, R. I. (2003). The cost efficiency of real estate investment trusts: an analysis with a Bayesian stochastic frontier model. The Journal of Real Estate Finance and Economics, 26(1), 65-80.

Ling, D. C., Ooi, J. T., \& Xu, R. (2016). Asset growth and stock performance: evidence from REITs. Real Estate Economics, DOI 10.1111/1540-6229.12186.

Linneman, P. (1997), Forces changing the real estate industry forever. Wharton Real Estate Review, Spring, 1-12.

McIntosh, W., \& Liang, Y. (1991). An examination of the small-firm effect within the REIT industry. Journal of Real Estate Research, 6(1), 9-17.

McIntosh, W., Ott, S. H., \& Liang, Y. (1995). The wealth effects of real estate transactions: the case of REITs. The Journal of Real Estate Finance and Economics, 10(3), 299-307.

Morri, G., \& Beretta, C. (2008). The capital structure determinants of REITs. Is it a peculiar industry? Journal of European Real Estate Research, 1(1), 6-57.

Mueller, G. (1998). REIT size and earnings growth: is bigger better, or a new challenge?. Journal of Real Estate Portfolio Management, 4(2), 149-157.

Norman, E., Sirmans, S., \& Benjamin, J. (1995). The historical environment of real estate returns. Journal of Real Estate Portfolio Management, 1(1), 1-24.

Rajan, R. G., \& Zingales, L. (1995). What do we know about capital structure? Some evidence from international data. The Journal of Finance, 50(5), 1421-1460.

Reinganum, M. R. (1981). Misspecification of capital asset pricing: empirical anomalies based on earnings' yields and market values. Journal of Financial Economics, 9(1), 1946. 
Ro, S., \& Ziobrowski, A. J. (2011). Does focus really matter? Specialized vs. diversified REITs. The Journal of Real Estate Finance and Economics, 42(1), 68-83.

Seguin, P. J. (2016). The relative value of public non-listed REITs, Journal of Real Estate Research, 38(1), 59-91.

Soyeh, K., \& Wiley, J. (2018). Liquidity management at REITs: listed \& public non-traded. Journal of Real Estate Research, forthcoming.

Tien, S., \& Sze, L. (2003). The role of Singapore REITs in a downside risk asset allocation framework. Journal of Real Estate Portfolio Management, 9(3), 219-235.

Titman, S., \& Wessels, R. (1988). The determinants of capital structure choice. The Journal of Finance, 43(1), 1-19.

Tom, G., \& Austin, J. (1996). Risk and real estate investment: an international perspective. Journal of Real Estate Research, 11(2), 117-130.

Vogel, J. H. (1997). Why the new conventional wisdom about REITs is wrong. Real Estate Finance, 14, 7-12.

Zardkoohi, A., \& Kolari, J. (1994). Branch office economies of scale and scope: evidence from savings banks in Finland. Journal of Banking and Finance, 18(3), 421-432. 
Table 1.1: Summary Statistics

This table reports the summary statistics of the key variables used in this paper. The sample period is from 1995 - 2016. All variables are defined in Appendix A1. Because the regression specification includes lagged variables, firms with fewer than two consecutive years of stock return and operational efficiency (OERl) information are excluded. Variables have been winsorized at the $1 \%$ and $99 \%$ tails of the distributions to avoid the influence of extreme observations.

\begin{tabular}{lcccccc}
\hline \hline & Mean & $\begin{array}{c}\text { Media } \\
\mathrm{n}\end{array}$ & $\begin{array}{c}\text { Std. } \\
\text { Dev. }\end{array}$ & Min & Max & $\begin{array}{c}\text { Yearly } \\
\text { Obs. }\end{array}$ \\
\hline Market Capitalization (\$B) & 2.270 & 0.903 & 3.975 & 0.005 & 24.136 & 3,426 \\
Total Assets (\$B) & 2.841 & 1.304 & 4.264 & 0.009 & 24.534 & 3,575 \\
Total Equity (\$B) & 1.111 & 0.492 & 1.661 & -0.056 & 9.180 & 3,575 \\
Total Debt (\$B) & 1.472 & 0.674 & 2.207 & 0.005 & 12.518 & 3,493 \\
Funds from Operations (\$B) & 0.143 & 0.066 & 0.225 & -0.067 & 1.301 & 3,514 \\
Total Revenue (\$B) & 0.422 & 0.183 & 0.664 & 0.001 & 4.028 & 3,575 \\
Expense Reimbursement (\$B) & 0.042 & 0.005 & 0.089 & 0.000 & 0.607 & 2,791 \\
Total Expense (\$B) & 0.373 & 0.152 & 0.601 & 0.000 & 3.642 & 3,575 \\
Real Estate Depreciation and & 0.090 & 0.037 & 0.139 & 0.000 & 0.786 & 3,575 \\
Amortization (\$B) & & & & & & \\
Rental Operating Expense (\$B) & 0.104 & 0.036 & 0.177 & 0.000 & 1.023 & 3,575 \\
EBITDA (\$B) & 0.243 & 0.113 & 0.398 & 0.000 & 5.559 & 3,479 \\
Leverage & 2.868 & 2.364 & 2.737 & -9.334 & 17.317 & 3,575 \\
Firm Age & 11.664 & 9.000 & 10.758 & 0.000 & 56.000 & 3,422 \\
Geographic Diversification & -0.426 & -0.310 & 0.283 & -1.000 & -0.119 & 2,901 \\
Property Type Diversification & -0.812 & -0.946 & 0.229 & -1.000 & -0.181 & 2,901 \\
Book-to-Market Ratio & 0.465 & 0.393 & 0.626 & -0.802 & 2.854 & 3,426 \\
Return on Assets (\%) & 6.036 & 6.051 & 3.920 & -7.229 & 18.261 & 3,201 \\
Return on Equity (\%) & 16.386 & 14.312 & 21.009 & -72.157 & 132.832 & 3,201 \\
Stock Return Volatility & 0.297 & 0.227 & 0.195 & 0.124 & 1.145 & 3,255 \\
EBITDA-to-Debt Ratio & 0.190 & 0.165 & 0.130 & 0.020 & 0.932 & 3,366 \\
Stock Return (\%) & 12.994 & 12.972 & 27.980 & -67.217 & 102.131 & 3,123 \\
OER1 & 0.995 & 0.956 & 0.338 & 0.276 & 2.579 & 3,575 \\
OER2 & 0.991 & 0.907 & 0.494 & 0.151 & 3.191 & 3,575 \\
\hline
\end{tabular}


Table 1.2: Return on Assets and Operational Efficiency

Panel A reports the results of multivariate regressions of REITs' return on assets (ROA) on their lagged standardized operational efficiency ratios (OER1 and OER2, respectively). The $t$-statistics are reported in brackets. The coefficients on variables of property type and years are suppressed from reporting. Standard errors are clustered at the firm level and are heteroscedasticity-robust. Panel B reports the time-series average of ROA of portfolios sorted by standardized operational efficiency ratios (OERI and OER2). The $t$-statistics from two-sample $t$-test with equal variances are reported in brackets. The $z$-statistics from two-sample Wilcoxon rank-sum test are reported in parentheses. Significance at the $1 \%$, $5 \%$ or $10 \%$ levels is shown with 3,2, or 1 asterisk, respectively. All variables are defined in Appendix A1. Because the regression specification includes lagged variables, firms with fewer than two consecutive years of stock return and operational efficiency (OERI) information are excluded. Variables have been winsorized at the $1 \%$ and $99 \%$ tails of the distributions to avoid the influence of extreme observations.

Panel A: Regressions

\begin{tabular}{|c|c|c|c|c|}
\hline VARIABLES & $\begin{array}{c}(1) \\
\mathrm{ROA}\end{array}$ & $\begin{array}{c}(2) \\
\text { ROA }\end{array}$ & $\begin{array}{c}(3) \\
\text { ROA }\end{array}$ & $\begin{array}{c}(4) \\
\text { ROA }\end{array}$ \\
\hline Log Market Capitalization, $t-1$ & $\begin{array}{c}0.398 \\
{[4.92]^{* * *}}\end{array}$ & $\begin{array}{c}0.463 \\
{[4.99]^{* * *}}\end{array}$ & $\begin{array}{c}0.723 \\
{[3.50]^{* * *}}\end{array}$ & $\begin{array}{c}0.793 \\
{[3.68]^{* * *}}\end{array}$ \\
\hline Leverage, $t-1$ & $\begin{array}{c}-0.067 \\
{[-1.76]^{*}}\end{array}$ & $\begin{array}{c}-0.082 \\
{[-2.08]^{* *}}\end{array}$ & $\begin{array}{c}-0.068 \\
{[-1.90]^{*}}\end{array}$ & $\begin{array}{c}-0.070 \\
{[-1.93]^{*}}\end{array}$ \\
\hline Firm Age, $t-1$ & $\begin{array}{l}-0.058 \\
{[-0.48]}\end{array}$ & $\begin{array}{l}-0.145 \\
{[-1.09]}\end{array}$ & $\begin{array}{c}-0.705 \\
{[-2.30]^{* *}}\end{array}$ & $\begin{array}{c}-0.710 \\
{[-2.24]^{* *}}\end{array}$ \\
\hline Geographic Diversification, $t-1$ & $\begin{array}{c}-1.074 \\
{[-3.21]^{* * *}}\end{array}$ & $\begin{array}{c}-0.983 \\
{[-2.57]^{* *}}\end{array}$ & $\begin{array}{c}-1.769 \\
{[-2.46]^{* *}}\end{array}$ & $\begin{array}{c}-1.804 \\
{[-2.21]^{* *}}\end{array}$ \\
\hline Property Type Diversification, $t-1$ & $\begin{array}{l}-0.704 \\
{[-1.25]}\end{array}$ & $\begin{array}{l}-0.335 \\
{[-0.55]}\end{array}$ & $\begin{array}{l}-0.139 \\
{[-0.16]}\end{array}$ & $\begin{array}{l}0.039 \\
{[0.04]}\end{array}$ \\
\hline Firm in S\&P Index, $t-1$ & $\begin{array}{l}0.247 \\
{[1.04]}\end{array}$ & $\begin{array}{l}0.157 \\
{[0.60]}\end{array}$ & $\begin{array}{l}-0.279 \\
{[-0.91]}\end{array}$ & $\begin{array}{l}-0.313 \\
{[-0.97]}\end{array}$ \\
\hline OER $1, t-1$ & $\begin{array}{c}-5.273 \\
{[-13.56] * * *}\end{array}$ & & $\begin{array}{c}-2.977 \\
{[-6.63]^{* * *}}\end{array}$ & \\
\hline$O E R 2, t-1$ & & $\begin{array}{c}-3.238 \\
{[-10.99]^{* * *}}\end{array}$ & & $\begin{array}{c}-1.760 \\
{[-6.17]^{* * *}}\end{array}$ \\
\hline Constant & $\begin{array}{c}7.679 \\
{[4.95]^{* * *}}\end{array}$ & $\begin{array}{c}5.893 \\
{[3.91]^{* * *}}\end{array}$ & $\begin{array}{c}7.828 \\
{[5.11]^{* * *}}\end{array}$ & $\begin{array}{c}6.434 \\
{[4.35]^{* * *}}\end{array}$ \\
\hline Observations & 2,494 & 2,494 & 2,494 & 2,494 \\
\hline Adj. R-sq. & 0.409 & 0.374 & 0.300 & 0.288 \\
\hline Property Type FE & YES & YES & NO & NO \\
\hline Firm FE & NO & NO & YES & YES \\
\hline Year FE & YES & YES & YES & YES \\
\hline Number of REIT & 249 & 249 & 249 & 249 \\
\hline
\end{tabular}




\begin{tabular}{ccc} 
Panel B: Quintiles sorting & \multicolumn{2}{c}{ ROA, $t+1$} \\
\cline { 2 - 3 } Ranking, $t$ & Rank by OER1 & Rank by OER2 \\
\cline { 2 - 3 } 1 & $8.08 / 7.81$ & $7.92 / 7.62$ \\
2 & $6.96 / 6.67$ & $6.67 / 6.46$ \\
3 & $6.30 / 6.14$ & $6.32 / 6.22$ \\
4 & $5.40 / 5.26$ & $5.55 / 5.51$ \\
5 & $3.34 / 3.55$ & $3.63 / 3.88$ \\
\hline $5-1$ Spread & $-4.74 /-4.27$ & $-4.29 /-3.73$ \\
$t$ test & {$[19.66] * * *$} & {$[17.42] * * *$} \\
rank-sum test & $(19.45) * * *$ & $(17.44) * * *$ \\
\hline
\end{tabular}


Table 1.3: Return on Equity and Operational Efficiency

Panel A reports the results of multivariate regressions of REITs' return on equity (ROE) on their lagged standardized operational efficiency ratios (OER1 and OER2, respectively). The $t$-statistics are reported in brackets. The coefficients on variables of property type and years are suppressed from reporting. Standard errors are clustered at the firm level and are heteroscedasticity-robust. Panel B reports the time-series average of ROE of portfolios sorted by standardized operational efficiency ratios (OERI and OER2). The $t$-statistics from two-sample $t$-test with equal variances are reported in brackets. The $z$-statistics from two-sample Wilcoxon rank-sum test are reported in parentheses. Significance at the $1 \%$, $5 \%$ or $10 \%$ levels is shown with 3,2, or 1 asterisks, respectively. All variables are defined in Appendix A1. Because the regression specification includes lagged variables, firms with fewer than two consecutive years of stock return and operational efficiency (OERI) information are excluded. Variables have been winsorized at the $1 \%$ and $99 \%$ tails of the distributions to avoid the influence of extreme observations.

\begin{tabular}{|c|c|c|c|c|}
\hline VARIABLES & $\begin{array}{c}(1) \\
\text { ROE }\end{array}$ & $\begin{array}{c}(2) \\
\text { ROE }\end{array}$ & $\begin{array}{c}(3) \\
\text { ROE }\end{array}$ & $\begin{array}{c}(4) \\
\text { ROE }\end{array}$ \\
\hline Log Market Capitalization, $t-1$ & $\begin{array}{l}0.638 \\
{[1.26]}\end{array}$ & $\begin{array}{l}0.790 \\
{[1.46]}\end{array}$ & $\begin{array}{l}-0.002 \\
{[-0.00]}\end{array}$ & $\begin{array}{l}0.079 \\
{[0.04]}\end{array}$ \\
\hline Leverage, $t-1$ & $\begin{array}{c}4.352 \\
{[5.49]^{* * *}}\end{array}$ & $\begin{array}{c}4.319 \\
{[5.49]^{* * *}}\end{array}$ & $\begin{array}{c}4.148 \\
{[4.22]^{* * *}}\end{array}$ & $\begin{array}{c}4.141 \\
{[4.22]^{* * *}}\end{array}$ \\
\hline Firm Age, $t-1$ & $\begin{array}{l}0.288 \\
{[0.49]}\end{array}$ & $\begin{array}{l}0.097 \\
{[0.16]}\end{array}$ & $\begin{array}{l}-2.350 \\
{[-1.20]}\end{array}$ & $\begin{array}{l}-2.410 \\
{[-1.24]}\end{array}$ \\
\hline Geographic Diversification, $t-1$ & $\begin{array}{c}-3.847 \\
{[-1.78]^{*}}\end{array}$ & $\begin{array}{c}-3.641 \\
{[-1.66]^{*}}\end{array}$ & $\begin{array}{c}-8.099 \\
{[-1.80]^{*}}\end{array}$ & $\begin{array}{c}-8.324 \\
{[-1.87]^{*}}\end{array}$ \\
\hline Property Type Diversification, $t-1$ & $\begin{array}{l}-4.200 \\
{[-1.30]}\end{array}$ & $\begin{array}{l}-3.369 \\
{[-1.08]}\end{array}$ & $\begin{array}{c}1.737 \\
{[0.26]}\end{array}$ & $\begin{array}{c}2.004 \\
{[0.31]}\end{array}$ \\
\hline Firm in S\&P Index, $t-1$ & $\begin{array}{l}0.027 \\
{[0.03]}\end{array}$ & $\begin{array}{l}-0.171 \\
{[-0.17]}\end{array}$ & $\begin{array}{l}-0.635 \\
{[-0.51]}\end{array}$ & $\begin{array}{l}-0.696 \\
{[-0.55]}\end{array}$ \\
\hline$O E R 1, t-1$ & $\begin{array}{c}-11.870 \\
{[-3.68] * * *}\end{array}$ & & $\begin{array}{c}-6.775 \\
{[-2.08] * *}\end{array}$ & \\
\hline$O E R 2, t-1$ & & $\begin{array}{c}-7.231 \\
{[-3.05] * * *}\end{array}$ & & $\begin{array}{c}-4.708 \\
{[-1.78]^{*}}\end{array}$ \\
\hline Constant & $\begin{array}{l}8.202 \\
{[0.94]}\end{array}$ & $\begin{array}{l}4.072 \\
{[0.47]}\end{array}$ & $\begin{array}{l}16.093 \\
{[1.35]}\end{array}$ & $\begin{array}{l}13.814 \\
{[1.15]}\end{array}$ \\
\hline Observations & 2,494 & 2,494 & 2,494 & 2,494 \\
\hline Adj. R-sq. & 0.397 & 0.392 & 0.301 & 0.301 \\
\hline Property Type FE & YES & YES & NO & NO \\
\hline Firm FE & NO & NO & YES & YES \\
\hline Year FE & YES & YES & YES & YES \\
\hline Number of REIT & 249 & 249 & 249 & 249 \\
\hline
\end{tabular}


Panel B: Quintiles sorting

ROE, $t+1$

\begin{tabular}{ccc}
\cline { 2 - 2 } Ranking, $t$ & Rank by OERI & Rank by OER2 \\
\hline 1 & $17.16 / 14.71$ & $17.86 / 15.06$ \\
2 & $17.80 / 15.43$ & $17.18 / 15.20$ \\
3 & $17.99 / 15.39$ & $17.62 / 15.06$ \\
4 & $17.26 / 14.61$ & $17.02 / 13.87$ \\
5 & $11.60 / 9.94$ & $12.15 / 10.39$ \\
\hline $5-1$ Spread & $-5.56 /-4.77$ & $-5.72 /-4.67$ \\
rank-sum test & {$[4.46] * * *$} & {$[4.57] * * *$} \\
\hline
\end{tabular}




\section{Table 1.4: Total Risk and Operational Efficiency}

Panel A reports the results of multivariate regressions of REITs' total risk, which is measured as its annualized stock return volatility, on their lagged standardized operational efficiency ratios (OERI and $O E R 2$, respectively). The $t$-statistics are reported in brackets. The coefficients on variables of property type and years are suppressed from reporting. Standard errors are clustered at the firm level and are heteroscedasticity-robust. Panel B reports the time-series average of stock return volatility of portfolios sorted by standardized operational efficiency ratios (OERI and $O E R 2)$. The $t$-statistics from two-sample $t$-test with equal variances are reported in brackets. The $z$-statistics from two-sample Wilcoxon rank-sum test are reported in parentheses. Significance at the $1 \%, 5 \%$ or $10 \%$ levels is shown with 3, 2, or 1 asterisks, respectively. All variables are defined in Appendix A1. Because the regression specification includes lagged variables, firms with fewer than two consecutive years of stock return and operational efficiency (OERI) information are excluded. Variables have been winsorized at the $1 \%$ and $99 \%$ tails of the distributions to avoid the influence of extreme observations.

Panel A: Regressions

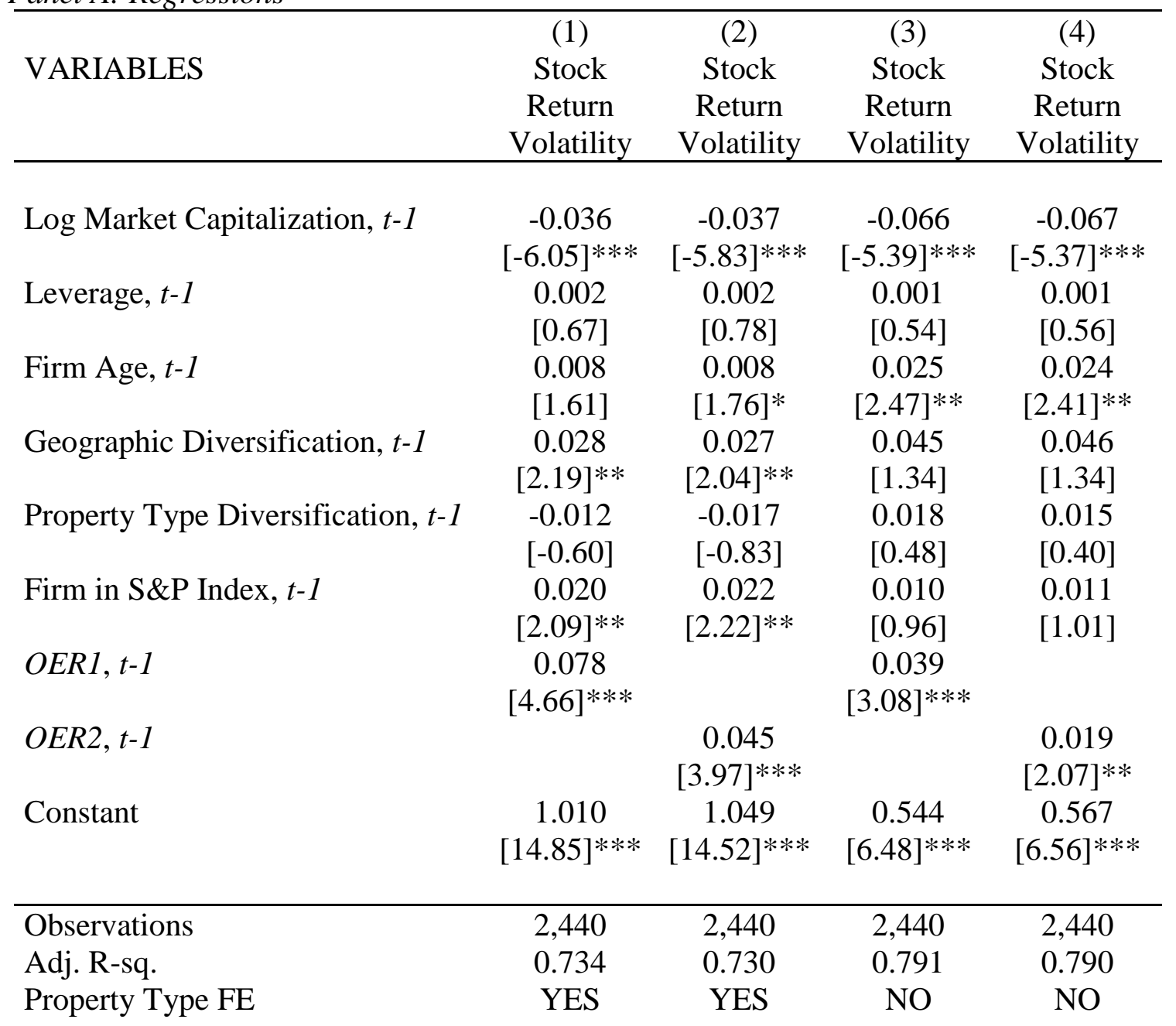


Firm FE

Year FE

NO

YES

NO

YES

YES

Number of REIT

242

YES

YES

YES

242

242

242

Panel B: Quintiles sorting

\begin{tabular}{ccc}
\hline & \multicolumn{2}{c}{ Stock Return Volatility, $t+1$} \\
\cline { 2 - 3 } Ranking, $t$ & Rank by OERl & Rank by OER2 \\
\hline 1 & $0.27 / 0.22$ & $0.27 / 0.22$ \\
2 & $0.27 / 0.21$ & $0.27 / 0.22$ \\
3 & $0.29 / 0.22$ & $0.29 / 0.22$ \\
4 & $0.30 / 0.23$ & $0.30 / 0.23$ \\
5 & $0.36 / 0.27$ & $0.35 / 0.26$ \\
\hline $5-1$ Spread & $0.09 / 0.05$ & $0.08 / 0.04$ \\
test & {$[-8.16] * * *$} & {$[-7.02] * * *$} \\
rank-sum test & $(-7.69) * * *$ & $(-7.20) * * *$ \\
\hline
\end{tabular}


Table 1.5: Credit Risk and Operational Efficiency

Panel A reports the results of multivariate regressions of REITs' Credit Risk, which is measured as EBITDA-to-Debt Ratio, on their lagged standardized operational efficiency ratios (OER1 and OER2, respectively). The $t$-statistics are reported in brackets. The coefficients on variables of property type and years are suppressed from reporting. Standard errors are clustered at the firm level and are heteroscedasticity-robust. Panel B reports the time-series average of EBITDA-to-Debt of portfolios sorted by standardized operational efficiency ratios (OERI and OER2). The $t$-statistics from two-sample $t$-test with equal variances are reported in brackets. The $z$-statistics from two-sample Wilcoxon rank-sum test are reported in parentheses. Significance at the $1 \%, 5 \%$ or $10 \%$ levels is shown with 3,2, or 1 asterisks, respectively. All variables are defined in Appendix A1. Because the regression specification includes lagged variables, firms with fewer than two consecutive years of stock return and operational efficiency (OERI) information are excluded. Variables have been winsorized at the $1 \%$ and $99 \%$ tails of the distributions to avoid the influence of extreme observations.

Panel A: Regressions

\begin{tabular}{|c|c|c|c|c|}
\hline VARIABLES & $\begin{array}{c}\text { (1) } \\
\text { EBITDA- } \\
\text { to-Debt }\end{array}$ & $\begin{array}{c}\text { (2) } \\
\text { EBITDA- } \\
\text { to-Debt }\end{array}$ & $\begin{array}{c}\text { (3) } \\
\text { EBITDA- } \\
\text { to-Debt }\end{array}$ & $\begin{array}{c}\text { (4) } \\
\text { EBITDA- } \\
\text { to-Debt }\end{array}$ \\
\hline Log Market Capitalization, $t-1$ & $\begin{array}{l}-0.003 \\
{[-0.88]}\end{array}$ & $\begin{array}{l}-0.002 \\
{[-0.63]}\end{array}$ & $\begin{array}{l}0.004 \\
{[0.41]}\end{array}$ & $\begin{array}{l}0.005 \\
{[0.53]}\end{array}$ \\
\hline Leverage, $t-1$ & $\begin{array}{c}-0.005 \\
{[-3.87]^{* * *}}\end{array}$ & $\begin{array}{c}-0.005 \\
{[-4.05] * * *}\end{array}$ & $\begin{array}{c}-0.003 \\
{[-3.35]^{* * *}}\end{array}$ & $\begin{array}{c}-0.003 \\
{[-3.35]^{* * *}}\end{array}$ \\
\hline Firm Age, $t-1$ & $\begin{array}{c}0.010 \\
{[1.99] * *}\end{array}$ & $\begin{array}{l}0.007 \\
{[1.44]}\end{array}$ & $\begin{array}{l}-0.003 \\
{[-0.32]}\end{array}$ & $\begin{array}{l}-0.004 \\
{[-0.37]}\end{array}$ \\
\hline Geographic Diversification, $t-1$ & $\begin{array}{l}-0.005 \\
{[-0.31]}\end{array}$ & $\begin{array}{l}-0.003 \\
{[-0.20]}\end{array}$ & $\begin{array}{l}0.049 \\
{[1.22]}\end{array}$ & $\begin{array}{l}0.047 \\
{[1.16]}\end{array}$ \\
\hline Property Type Diversification, $t-1$ & $\begin{array}{l}-0.025 \\
{[-1.12]}\end{array}$ & $\begin{array}{l}-0.018 \\
{[-0.79]}\end{array}$ & $\begin{array}{l}0.000 \\
{[0.01]}\end{array}$ & $\begin{array}{l}0.002 \\
{[0.09]}\end{array}$ \\
\hline Firm in S\&P Index, $t-1$ & $\begin{array}{c}0.023 \\
{[1.86]^{*}}\end{array}$ & $\begin{array}{l}0.021 \\
{[1.64]}\end{array}$ & $\begin{array}{l}-0.017 \\
{[-1.47]}\end{array}$ & $\begin{array}{l}-0.017 \\
{[-1.52]}\end{array}$ \\
\hline OERl, $t-1$ & $\begin{array}{c}-0.134 \\
{[-6.25]^{* * *}}\end{array}$ & & $\begin{array}{c}-0.056 \\
{[-3.30] * * *}\end{array}$ & \\
\hline OER2, $t-1$ & & $\begin{array}{c}-0.086 \\
{[-5.76] * * *}\end{array}$ & & $\begin{array}{c}-0.035 \\
{[-3.34] * * *}\end{array}$ \\
\hline Constant & $\begin{array}{c}0.203 \\
{[4.10]^{* * *}}\end{array}$ & $\begin{array}{c}0.153 \\
{[3.42]^{* * *}}\end{array}$ & $\begin{array}{c}0.306 \\
{[3.77]^{* * *}}\end{array}$ & $\begin{array}{c}0.280 \\
{[3.53]^{* * *}}\end{array}$ \\
\hline Observatiol & 2,463 & 2,463 & 2,463 & 2,463 \\
\hline Adj. R-sq. & 0.229 & 0.221 & 0.101 & 0.099 \\
\hline Property Type FE & YES & YES & NO & NO \\
\hline Firm FE & NO & NO & YES & YES \\
\hline
\end{tabular}


Year FE

YES

YES

YES

YES

Number of REIT

247

247

247

247

Panel B: Quintiles sorting

\begin{tabular}{ccc} 
& \multicolumn{2}{c}{ EBITDA-to-Debt, $t+1$} \\
\cline { 2 - 3 } Ranking, $t$ & Rank by OER1 & Rank by OER2 \\
\hline 1 & $0.26 / 0.20$ & $0.26 / 0.20$ \\
2 & $0.19 / 0.18$ & $0.19 / 0.17$ \\
3 & $0.18 / 0.16$ & $0.18 / 0.17$ \\
4 & $0.17 / 0.15$ & $0.17 / 0.15$ \\
5 & $0.15 / 0.13$ & $0.15 / 0.13$ \\
\hline $5-1$ Spread & $-0.11 /-0.07$ & $-0.10 /-0.06$ \\
t test & {$[12.67] * * *$} & {$[11.27] * * *$} \\
rank-sum test & $(15.88) * * *$ & $(14.19) * * *$ \\
\hline
\end{tabular}


Table 1.6: Cross-Sectional Stock Return and Operational Efficiency

This table presents OLS regression coefficient estimates of REITs' annual excess return on the Fama French (1993) three factors, Carhart (1997) momentum factor and Fama French (2015) five factors, and a REIT' lagged standardized operational efficiency ratios (OER1 and $O E R 2$, respectively). The $t$-statistics are reported in brackets. Standard errors are clustered at the firm level and are heteroscedasticity-robust. All variables are defined in Appendix A1. Significance at the $1 \%, 5 \%$ or $10 \%$ levels is shown with 3,2, or 1 asterisks, respectively. Because the regression specification includes lagged variables, firms with fewer than two consecutive years of stock return and operational efficiency (OERI) information are excluded. Variables have been winsorized at the 1\% and $99 \%$ tails of the distributions to avoid the influence of extreme observations.

\begin{tabular}{|c|c|c|c|c|c|c|}
\hline VARIABLES & $\begin{array}{c}\text { (1) } \\
\text { Excess } \\
\text { Return } \\
\end{array}$ & $\begin{array}{c}\text { (2) } \\
\text { Excess } \\
\text { Return } \\
\end{array}$ & $\begin{array}{c}\text { (3) } \\
\text { Excess } \\
\text { Return } \\
\end{array}$ & $\begin{array}{c}\text { (4) } \\
\text { Excess } \\
\text { Return }\end{array}$ & $\begin{array}{c}(5) \\
\text { Excess } \\
\text { Return } \\
\end{array}$ & $\begin{array}{c}\text { (6) } \\
\text { Excess } \\
\text { Return }\end{array}$ \\
\hline mktrf & 0.546 & 0.469 & 0.725 & 0.546 & 0.468 & 0.725 \\
\hline & {$[17.50]^{* * *}$} & {$[14.48] * * *$} & {$[16.92] * * *$} & {$[17.45]^{* * *}$} & {$[14.50]^{* * *}$} & {$[16.96]^{* * *}$} \\
\hline $\mathrm{smb}$ & 0.544 & 0.455 & 0.674 & 0.544 & 0.454 & 0.673 \\
\hline & {$[15.32]^{* * *}$} & {$[12.69] * * *$} & {$[14.18]^{* * *}$} & {$[15.32] * * *$} & {$[12.65]^{* * *}$} & {$[14.21]^{* * *}$} \\
\hline $\mathrm{hml}$ & $\begin{array}{c}0.543 \\
{[20.92]^{* * *}}\end{array}$ & $\begin{array}{c}0.499 \\
{[17.51]^{* * *}}\end{array}$ & $\begin{array}{c}0.288 \\
{[5.48]^{* * *}}\end{array}$ & $\begin{array}{c}0.543 \\
{[20.91]^{* * *}}\end{array}$ & $\begin{array}{c}0.499 \\
{[17.47]^{* * *}}\end{array}$ & $\begin{array}{c}0.288 \\
{[5.49]^{* * *}}\end{array}$ \\
\hline mom & & $\begin{array}{c}-0.144 \\
{[-5.07]^{* * *}}\end{array}$ & & & $\begin{array}{c}-0.145 \\
{[-5.07] * * *}\end{array}$ & \\
\hline $\mathrm{rmw}$ & & & $\begin{array}{c}0.476 \\
{[7.11]^{* * *}}\end{array}$ & & & $\begin{array}{c}0.475 \\
{[7.13]^{* * *}}\end{array}$ \\
\hline $\mathrm{cma}$ & & & $\begin{array}{l}0.075 \\
{[1.12]}\end{array}$ & & & $\begin{array}{l}0.075 \\
{[1.12]}\end{array}$ \\
\hline OER 1 & $\begin{array}{c}-9.738 \\
{[-4.89] * * *}\end{array}$ & $\begin{array}{c}-9.674 \\
{[-4.84]^{* * *}}\end{array}$ & $\begin{array}{c}-9.619 \\
{[-4.81] * * *}\end{array}$ & & & \\
\hline OER2 & & & & $\begin{array}{c}-6.330 \\
{[-4.39]^{* * *}}\end{array}$ & $\begin{array}{c}-6.301 \\
{[-4.36]^{* * *}}\end{array}$ & $\begin{array}{c}-6.228 \\
{[-4.28]^{* * *}}\end{array}$ \\
\hline Constant & $\begin{array}{c}12.425 \\
{[6.76]^{* * *}}\end{array}$ & $\begin{array}{c}14.071 \\
{[7.41]^{* * *}}\end{array}$ & $\begin{array}{c}9.392 \\
{[4.67]^{* * *}}\end{array}$ & $\begin{array}{c}9.021 \\
{[6.78]^{* * *}}\end{array}$ & $\begin{array}{c}10.708 \\
{[7.65]^{* * *}}\end{array}$ & $\begin{array}{c}6.010 \\
{[4.04]^{* * *}}\end{array}$ \\
\hline Observations & 3,123 & 3,123 & 3,123 & 3,123 & 3,123 & 3,123 \\
\hline Adj. R-sq. & 0.238 & 0.248 & 0.251 & 0.237 & 0.246 & 0.250 \\
\hline
\end{tabular}


Table 1.7: Residual Stock Return and Operational Efficiency

This table reports the results of multivariate regressions of REITs' residual excess stock return (Residual Return), which is obtained from the Fama-French (2015) five-factor model, on their lagged standardized operational efficiency ratios (OER1 and OER2, respectively). The $t$-statistics are reported in brackets. The coefficients on variables of property type and years are suppressed from reporting. Standard errors are clustered at the firm level and are heteroscedasticity-robust. Significance at the $1 \%, 5 \%$ or $10 \%$ levels is shown with 3, 2, or 1 asterisks, respectively. All variables are defined in Appendix A1. Because the regression specification includes lagged variables, firms with fewer than two consecutive years of stock return and operational efficiency (OERl) information are excluded. Variables have been winsorized at the $1 \%$ and $99 \%$ tails of the distributions to avoid the influence of extreme observations.

\begin{tabular}{|c|c|c|c|c|}
\hline VARIABLES & $\begin{array}{c}\text { (1) } \\
\text { Residual } \\
\text { Return }\end{array}$ & $\begin{array}{c}\text { (2) } \\
\text { Residual } \\
\text { Return } \\
\end{array}$ & $\begin{array}{c}\text { (3) } \\
\text { Residual } \\
\text { Return } \\
\end{array}$ & $\begin{array}{c}\text { (4) } \\
\text { Residual } \\
\text { Return } \\
\end{array}$ \\
\hline Log Market Capitalization, $t-1$ & $\begin{array}{c}-0.884 \\
{[-2.57] * *}\end{array}$ & $\begin{array}{c}-0.749 \\
{[-2.10]^{* *}}\end{array}$ & $\begin{array}{c}-13.394 \\
{[-10.09]^{* * *}}\end{array}$ & $\begin{array}{c}-13.149 \\
{[-9.47] * * *}\end{array}$ \\
\hline Leverage, $t-1$ & $\begin{array}{c}0.090 \\
{[0.37]}\end{array}$ & $\begin{array}{l}0.061 \\
{[0.25]}\end{array}$ & $\begin{array}{l}-0.180 \\
{[-0.68]}\end{array}$ & $\begin{array}{l}-0.183 \\
{[-0.69]}\end{array}$ \\
\hline Firm Age, $t-1$ & $\begin{array}{c}-1.310 \\
{[-2.28] * *}\end{array}$ & $\begin{array}{c}-1.469 \\
{[-2.46]^{* *}}\end{array}$ & $\begin{array}{l}0.575 \\
{[0.32]}\end{array}$ & $\begin{array}{l}0.611 \\
{[0.34]}\end{array}$ \\
\hline Geographic Diversification, $t-1$ & $\begin{array}{l}-1.250 \\
{[-0.84]}\end{array}$ & $\begin{array}{l}-1.045 \\
{[-0.68]}\end{array}$ & $\begin{array}{l}2.715 \\
{[0.45]}\end{array}$ & $\begin{array}{c}2.769 \\
{[0.45]}\end{array}$ \\
\hline Property Type Diversification, $t-1$ & $\begin{array}{l}-0.881 \\
{[-0.38]}\end{array}$ & $\begin{array}{l}-0.089 \\
{[-0.04]}\end{array}$ & $\begin{array}{l}-0.327 \\
{[-0.06]}\end{array}$ & $\begin{array}{l}0.203 \\
{[0.04]}\end{array}$ \\
\hline Firm in S\&P Index, $t-1$ & $\begin{array}{l}-1.393 \\
{[-1.23]}\end{array}$ & $\begin{array}{l}-1.579 \\
{[-1.37]}\end{array}$ & $\begin{array}{l}-1.385 \\
{[-0.82]}\end{array}$ & $\begin{array}{l}-1.490 \\
{[-0.88]}\end{array}$ \\
\hline OER $1, t-1$ & $\begin{array}{c}-10.628 \\
{[-4.16]^{* * *}}\end{array}$ & & $\begin{array}{c}-7.108 \\
{[-2.02]^{* *}}\end{array}$ & \\
\hline$O E R 2, t-1$ & & $\begin{array}{c}-6.448 \\
{[-3.74]^{* * *}}\end{array}$ & & $\begin{array}{c}-3.570 \\
{[-1.70]^{*}}\end{array}$ \\
\hline Constant & $\begin{array}{c}15.515 \\
{[2.62]^{* * *}}\end{array}$ & $\begin{array}{r}10.842 \\
{[1.96]^{*}}\end{array}$ & $\begin{array}{c}81.297 \\
{[8.24]^{* * *}}\end{array}$ & $\begin{array}{c}77.145 \\
{[8.00]^{* * *}}\end{array}$ \\
\hline Observations & 2,537 & 2,537 & 2,537 & 2,537 \\
\hline Adj. R-sq. & 0.425 & 0.422 & 0.477 & 0.475 \\
\hline Property Type FE & YES & YES & NO & NO \\
\hline Firm FE & NO & NO & YES & YES \\
\hline Year FE & YES & YES & YES & YES \\
\hline Number of REIT & 250 & 250 & 250 & 250 \\
\hline
\end{tabular}


Table 1.8: Robustness check

This table presents the results of robustness checks. Panel A reports correlations of regression variables on the relationship between performance and operational efficiency measures at year $t$ and year $t-1$. Significance at the 5\% levels is shown with one asterisk. Panel B reports the results of regressing the performance (ROA and ROE) of REIT on their standardized operational efficiency ratios (OER 1 and $O E R 2$, respectively) at the same period. The $t$-statistics are reported in brackets. The coefficients on variables of property type and years are suppressed from reporting. Standard errors are clustered at the firm level and are heteroscedasticity-robust. Significance at the $1 \%, 5 \%$ or $10 \%$ levels is shown with 3, 2, or 1 asterisks, respectively. All variables are defined in Appendix A1. Because the regression specification includes lagged variables, firms with fewer than two consecutive years of stock return and operational efficiency $(O E R 1)$ information are excluded. Variables have been winsorized at the $1 \%$ and $99 \%$ tails of the distributions to avoid the influence of extreme observations.

Panel A: correlations of key regression variables at year $t$ and at year $t-1$

\begin{tabular}{|c|c|c|c|c|c|c|c|c|}
\hline & $\mathrm{ROA}, t$ & $\begin{array}{c}\mathrm{ROA}, t- \\
1\end{array}$ & ROE, $t$ & $\begin{array}{c}\mathrm{ROE}, t- \\
1\end{array}$ & $\begin{array}{c}\text { Market } \\
\text { Capitali } \\
\text { zation, } t\end{array}$ & $\begin{array}{c}\text { Market } \\
\text { Capitaliza } \\
\text { tion, } t-1\end{array}$ & $\begin{array}{l}\text { Leverag } \\
\text { e, } t\end{array}$ & $\begin{array}{l}\text { Leverag } \\
\mathrm{e}, t-1\end{array}$ \\
\hline $\mathrm{ROA}, t$ & 1 & & & & & & & \\
\hline $\mathrm{ROA}, t-1$ & $0.678 *$ & 1 & & & & & & \\
\hline ROE, $t$ & $0.433 *$ & $0.226^{*}$ & 1 & & & & & \\
\hline ROE, $t-1$ & $0.235^{*}$ & $0.416^{*}$ & $0.534 *$ & 1 & & & & \\
\hline Market Capitalization, $t$ & $0.097 *$ & $0.075^{*}$ & $0.041^{*}$ & 0.030 & 1 & & & \\
\hline Market Capitalization, $t-1$ & $0.084 *$ & $0.082 *$ & $0.041 *$ & 0.036 & $0.966^{*}$ & 1 & & \\
\hline Leverage, $t$ & $-0.102 *$ & $-0.081^{*}$ & $0.336^{*}$ & $0.307 *$ & -0.007 & -0.009 & 1 & \\
\hline Leverage, $t-1$ & $-0.119 *$ & $-0.108 *$ & $0.522 *$ & $0.355^{*}$ & -0.004 & 0.003 & $0.616^{*}$ & 1 \\
\hline Firm Age, $t$ & $-0.093 *$ & $-0.103 *$ & $-0.049 *$ & $-0.056^{*}$ & $0.151^{*}$ & $0.129 *$ & -0.013 & -0.014 \\
\hline Firm Age, $t-1$ & $-0.088 *$ & $-0.108 *$ & $-0.043 *$ & $-0.059 *$ & $0.135^{*}$ & $0.136^{*}$ & -0.027 & -0.010 \\
\hline GeogDiverse, $t$ & $0.047 *$ & $0.056^{*}$ & -0.020 & -0.012 & $0.159 *$ & $0.152 *$ & -0.0291 & -0.0360 \\
\hline GeogDiverse, $t-1$ & 0.027 & $0.046^{*}$ & -0.029 & -0.017 & $0.166^{*}$ & $0.163 *$ & -0.035 & -0.030 \\
\hline
\end{tabular}




\begin{tabular}{|l|c|c|c|c|c|c|c|c|}
\hline PropTypeDiverse, $t$ & -0.038 & -0.032 & -0.010 & -0.011 & 0.010 & 0.020 & 0.0284 & 0.028 \\
\hline PropTypeDiverse, $t-1$ & $-0.054^{*}$ & $-0.042^{*}$ & -0.021 & -0.015 & -0.001 & 0.012 & $0.038^{*}$ & 0.026 \\
\hline OER $1, t$ & $-0.657^{*}$ & $-0.428^{*}$ & $-0.248^{*}$ & $-0.121^{*}$ & $-0.109^{*}$ & $-0.103^{*}$ & $0.103^{*}$ & $0.109^{*}$ \\
\hline OER $1, t-1$ & $-0.431^{*}$ & $-0.636^{*}$ & $-0.094^{*}$ & $-0.228^{*}$ & $-0.108^{*}$ & $-0.096^{*}$ & $0.087^{*}$ & $0.109^{*}$ \\
\hline OER $2, t$ & $-0.610^{*}$ & $-0.389^{*}$ & $-0.249^{*}$ & $-0.130^{*}$ & $-0.122^{*}$ & $-0.117^{*}$ & $0.060^{*}$ & $0.057^{*}$ \\
\hline OER $2, t-1$ & $-0.395^{*}$ & $-0.589^{*}$ & $-0.100^{*}$ & $-0.229 *$ & $-0.116^{*}$ & $-0.109 *$ & $0.056^{*}$ & $0.065^{*}$ \\
\hline
\end{tabular}

Panel A: correlations of key regression variables at year $t$ and at year $t-1$ (Continued)

\begin{tabular}{|c|c|c|c|c|c|c|c|c|c|c|}
\hline & $\begin{array}{c}\text { Firm } \\
\text { Age, } t\end{array}$ & $\begin{array}{l}\text { Firm } \\
\text { Age, } \\
t-1\end{array}$ & $\begin{array}{c}\text { Geog } \\
\text { Divers } \\
\mathrm{e}, t\end{array}$ & $\begin{array}{c}\text { Geog } \\
\text { Divers } \\
\text { e, } t-1\end{array}$ & $\begin{array}{c}\text { PropT } \\
\text { ypeDi } \\
\text { verse, } \\
t\end{array}$ & \begin{tabular}{|c|} 
PropT \\
ypeDi \\
verse, \\
$t-1$ \\
\end{tabular} & $\begin{array}{c}\text { OER1, } \\
t\end{array}$ & $\begin{array}{c}O E R 1 \\
t-1\end{array}$ & $\begin{array}{c}O E R 2 \\
t\end{array}$ & $\begin{array}{c}\text { OER2, } \\
t-1\end{array}$ \\
\hline Firm Age, $t$ & 1 & & & & & & & & & \\
\hline Firm Age, $t$ - 1 & -0.014 & 1 & & & & & & & & \\
\hline GeogDiverse, $t$ & -0.010 & $0.999 *$ & 1 & & & & & & & \\
\hline GeogDiverse, $t-1$ & -0.0360 & $-0.061 *$ & $0.966^{*}$ & 1 & & & & & & \\
\hline PropTypeDiverse, $t$ & -0.030 & $-0.059 *$ & $-0.102 *$ & $-0.117 *$ & 1 & & & & & \\
\hline PropTypeDiverse, $t-1$ & 0.028 & $0.190 *$ & $-0.126^{*}$ & $-0.102 *$ & $0.956 *$ & 1 & & & & \\
\hline$O E R 1, t$ & 0.026 & $0.188 *$ & $-0.084 *$ & $-0.062 *$ & $-0.095 *$ & $-0.056 *$ & 1 & & & \\
\hline OER1, $t-1$ & $0.109 *$ & $-0.099 *$ & $-0.076 *$ & $-0.087 *$ & $-0.089 *$ & $-0.100 *$ & $0.633^{*}$ & 1 & & \\
\hline$O E R 2, t$ & $0.109 *$ & $-0.112 *$ & $-0.070 *$ & $-0.042 *$ & $-0.043 *$ & -0.006 & $0.865^{*}$ & $0.523^{*}$ & 1 & \\
\hline$O E R 2, t-1$ & $0.057 *$ & $-0.127 *$ & $-0.062 *$ & $-0.070 *$ & -0.033 & $-0.043 *$ & $0.522 *$ & $0.860 *$ & $0.641 *$ & 1 \\
\hline
\end{tabular}


Panel B: Performance and operational efficiency

\begin{tabular}{|c|c|c|c|c|c|c|c|c|}
\hline VARIABLES & $\begin{array}{c}(1) \\
\text { ROA } \\
\end{array}$ & $\begin{array}{r}(2) \\
\text { ROA } \\
\end{array}$ & $\begin{array}{r}(3) \\
\text { ROA } \\
\end{array}$ & $\begin{array}{r}(4) \\
\text { ROA } \\
\end{array}$ & $\begin{array}{c}(5) \\
\text { ROE } \\
\end{array}$ & $\begin{array}{c}(6) \\
\text { ROE } \\
\end{array}$ & $\begin{array}{c}(7) \\
\text { ROE }\end{array}$ & $\begin{array}{c}(8) \\
\text { ROE } \\
\end{array}$ \\
\hline Log Market Capitalization, $t$ & $\begin{array}{c}0.274 \\
{[3.96]^{* * *}}\end{array}$ & $\begin{array}{c}0.329 \\
{[4.57]^{* * *}}\end{array}$ & $\begin{array}{c}0.905 \\
{[5.76]^{* * *}}\end{array}$ & $\begin{array}{c}1.022 \\
{[5.80]^{* * *}}\end{array}$ & $\begin{array}{l}0.327 \\
{[0.75]}\end{array}$ & $\begin{array}{l}0.396 \\
{[0.86]}\end{array}$ & $\begin{array}{l}0.223 \\
{[0.14]}\end{array}$ & $\begin{array}{l}0.378 \\
{[0.23]}\end{array}$ \\
\hline Leverage, $t$ & $\begin{array}{l}-0.030 \\
{[-0.84]}\end{array}$ & $\begin{array}{l}-0.050 \\
{[-1.42]}\end{array}$ & $\begin{array}{l}-0.044 \\
{[-1.43]}\end{array}$ & $\begin{array}{l}-0.052 \\
{[-1.60]}\end{array}$ & $\begin{array}{c}2.701 \\
{[4.86]^{* * *}}\end{array}$ & $\begin{array}{c}2.658 \\
{[4.90]^{* * *}}\end{array}$ & $\begin{array}{c}1.671 \\
{[2.58]^{* *}}\end{array}$ & $\begin{array}{c}1.649 \\
{[2.57]^{* *}}\end{array}$ \\
\hline Firm Age, $t$ & $\begin{array}{l}0.016 \\
{[0.13]}\end{array}$ & $\begin{array}{l}-0.157 \\
{[-1.20]}\end{array}$ & $\begin{array}{l}-0.264 \\
{[-0.74]}\end{array}$ & $\begin{array}{l}-0.306 \\
{[-0.80]}\end{array}$ & $\begin{array}{l}-0.045 \\
{[-0.06]}\end{array}$ & $\begin{array}{l}-0.500 \\
{[-0.64]}\end{array}$ & $\begin{array}{l}-1.750 \\
{[-0.55]}\end{array}$ & $\begin{array}{l}-1.851 \\
{[-0.59]}\end{array}$ \\
\hline Geographic Diversification, $t$ & $\begin{array}{c}-0.828 \\
{[-2.73]^{* * *}}\end{array}$ & $\begin{array}{c}-0.694 \\
{[-2.04]^{* *}}\end{array}$ & $\begin{array}{c}-1.313 \\
{[-2.53]^{* *}}\end{array}$ & $\begin{array}{c}-1.541 \\
{[-2.21]^{* *}}\end{array}$ & $\begin{array}{c}-3.711 \\
{[-1.73]^{*}}\end{array}$ & $\begin{array}{l}-3.370 \\
{[-1.50]}\end{array}$ & $\begin{array}{l}-1.076 \\
{[-0.20]}\end{array}$ & $\begin{array}{l}-1.893 \\
{[-0.35]}\end{array}$ \\
\hline Property Type Diversification, $t$ & $\begin{array}{l}-0.387 \\
{[-0.73]}\end{array}$ & $\begin{array}{l}0.052 \\
{[0.10]}\end{array}$ & $\begin{array}{l}-0.013 \\
{[-0.02]}\end{array}$ & $\begin{array}{c}0.165 \\
{[0.23]}\end{array}$ & $\begin{array}{l}-2.752 \\
{[-0.67]}\end{array}$ & $\begin{array}{l}-1.667 \\
{[-0.43]}\end{array}$ & $\begin{array}{l}4.552 \\
{[0.43]}\end{array}$ & $\begin{array}{l}4.957 \\
{[0.50]}\end{array}$ \\
\hline Firm in S\&P Index, $t$ & $\begin{array}{l}0.207 \\
{[1.05]}\end{array}$ & $\begin{array}{l}0.071 \\
{[0.30]}\end{array}$ & $\begin{array}{c}-0.511 \\
{[-2.00]^{* *}}\end{array}$ & $\begin{array}{c}-0.598 \\
{[-2.11]^{* *}}\end{array}$ & $\begin{array}{l}-0.794 \\
{[-0.95]}\end{array}$ & $\begin{array}{l}-1.151 \\
{[-1.28]}\end{array}$ & $\begin{array}{l}-1.504 \\
{[-1.19]}\end{array}$ & $\begin{array}{l}-1.760 \\
{[-1.40]}\end{array}$ \\
\hline$O E R 1, t$ & $\begin{array}{c}-7.745 \\
{[-23.21]^{* * *}}\end{array}$ & & $\begin{array}{c}-7.224 \\
{[-16.29] * * *}\end{array}$ & & $\begin{array}{c}-18.614 \\
{[-5.23]^{* * *}}\end{array}$ & & $\begin{array}{c}-20.816 \\
{[-5.22]^{* * *}}\end{array}$ & \\
\hline$O E R 2, t$ & & $\begin{array}{c}-5.027 \\
{[-22.59]^{* * *}}\end{array}$ & & $\begin{array}{c}-4.546 \\
{[-19.37]^{* * *}}\end{array}$ & & $\begin{array}{c}-12.658 \\
{[-5.01]^{* * *}}\end{array}$ & & $\begin{array}{c}-14.657 \\
{[-4.94]^{* * *}}\end{array}$ \\
\hline Constant & $\begin{array}{c}15.672 \\
{[14.36]^{* * *}}\end{array}$ & $\begin{array}{c}13.628 \\
{[13.70]^{* * *}}\end{array}$ & $\begin{array}{c}10.179 \\
{[7.31]^{* * *}}\end{array}$ & $\begin{array}{c}7.079 \\
{[5.05]^{* * *}}\end{array}$ & $\begin{array}{c}27.059 \\
{[3.43]^{* * *}}\end{array}$ & $\begin{array}{c}23.246 \\
{[3.08]^{* * *}}\end{array}$ & $\begin{array}{c}39.681 \\
{[2.58]^{* *}}\end{array}$ & $\begin{array}{c}32.987 \\
{[2.18]^{* *}}\end{array}$ \\
\hline Obse & 2,555 & 2,555 & 2,555 & 2,555 & 2,555 & 2,555 & 2,555 & 2,555 \\
\hline Adj. R-sq. & 0.603 & 0.564 & 0.548 & 0.515 & 0.227 & 0.226 & 0.107 & 0.111 \\
\hline Property Type FE & YES & YES & NO & NO & YES & YES & NO & NO \\
\hline Firm FE & NO & NO & YES & YES & NO & NO & YES & YES \\
\hline Year FE & YES & YES & YES & YES & YES & YES & YES & YES \\
\hline Number of REIT & 247 & 247 & 247 & 247 & 247 & 247 & 247 & 247 \\
\hline
\end{tabular}



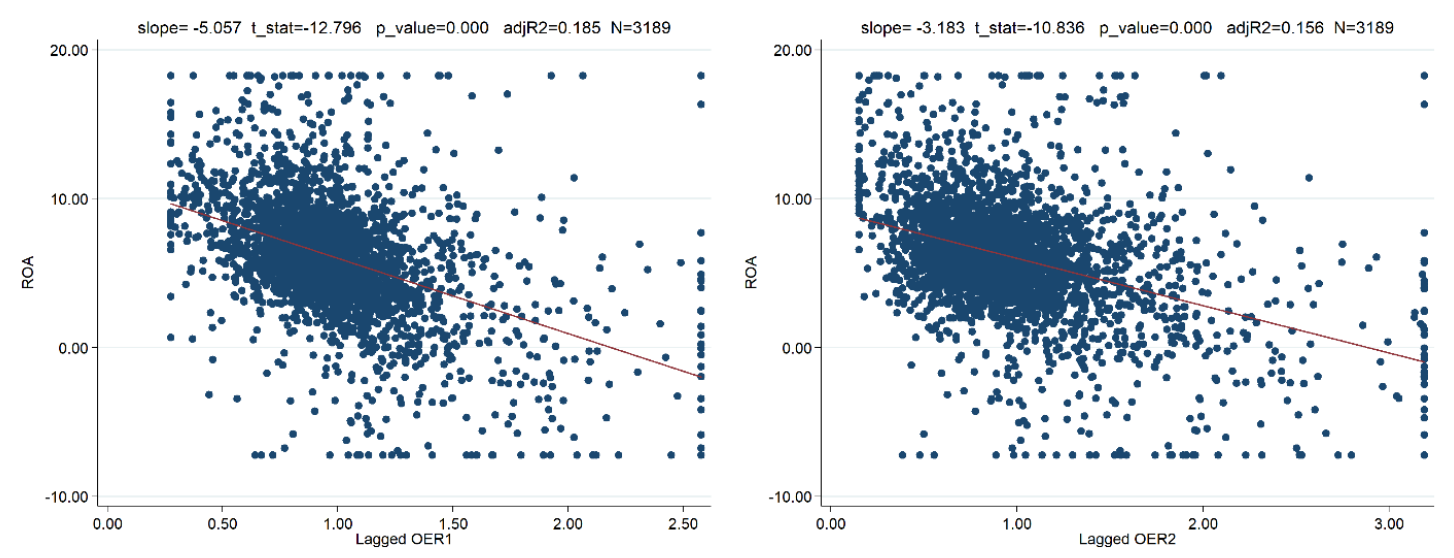

Panel A: ROA and OER
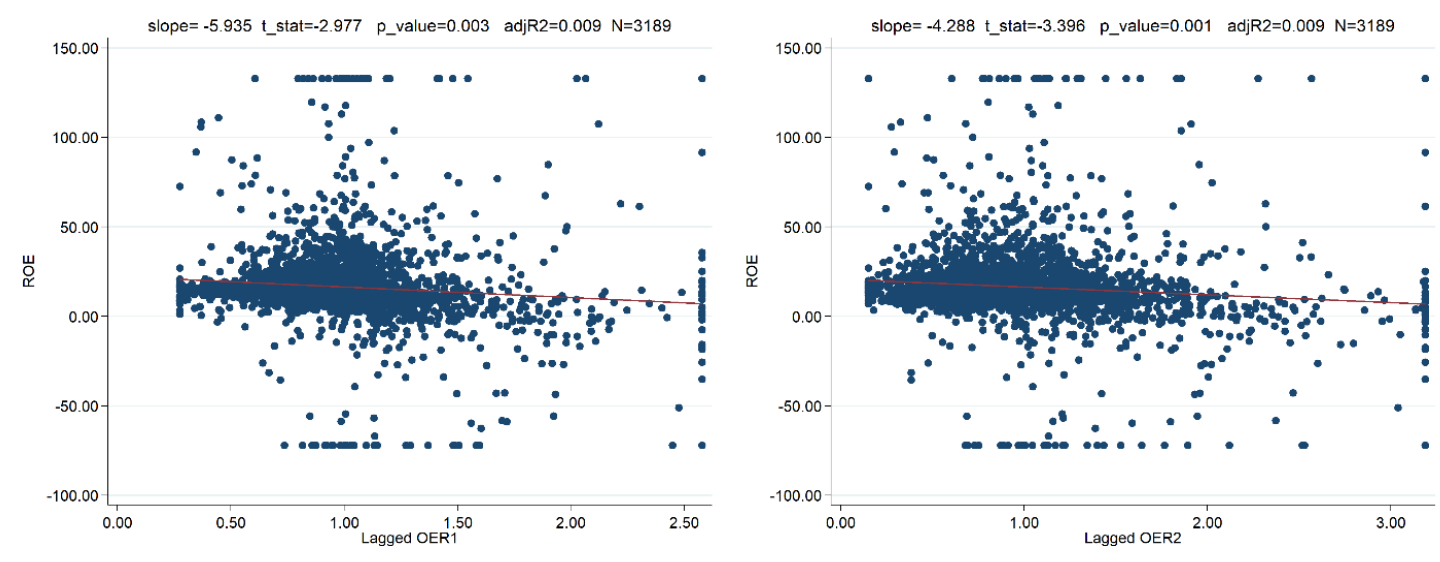

Panel B: ROE and OER

Figure 1.1: Operational Performance and Operational Efficiency

This figure plots return on assets (ROA) and return on equity (ROE) on the vertical axis against two lagged standardized operational efficiency ratios (OERI and OER2, respectively) on the horizontal axis for the sample period (1995 - 2016). The slope, $t$ statistics, $p$-value and adjusted $R$-squared are reported on the top of each figure. Standard errors are clustered at the firm level and are heteroscedasticity-robust. Significance at the $1 \%, 5 \%$ or $10 \%$ levels is shown with 3,2, or 1 asterisks, respectively. All variables are defined in Appendix A1. Because the regression specification includes lagged variables, firms with fewer than two consecutive years of stock return and operational efficiency (OERI) information are excluded. Variables have been winsorized at the $1 \%$ and $99 \%$ tails of the distributions to avoid the influence of extreme observations. 

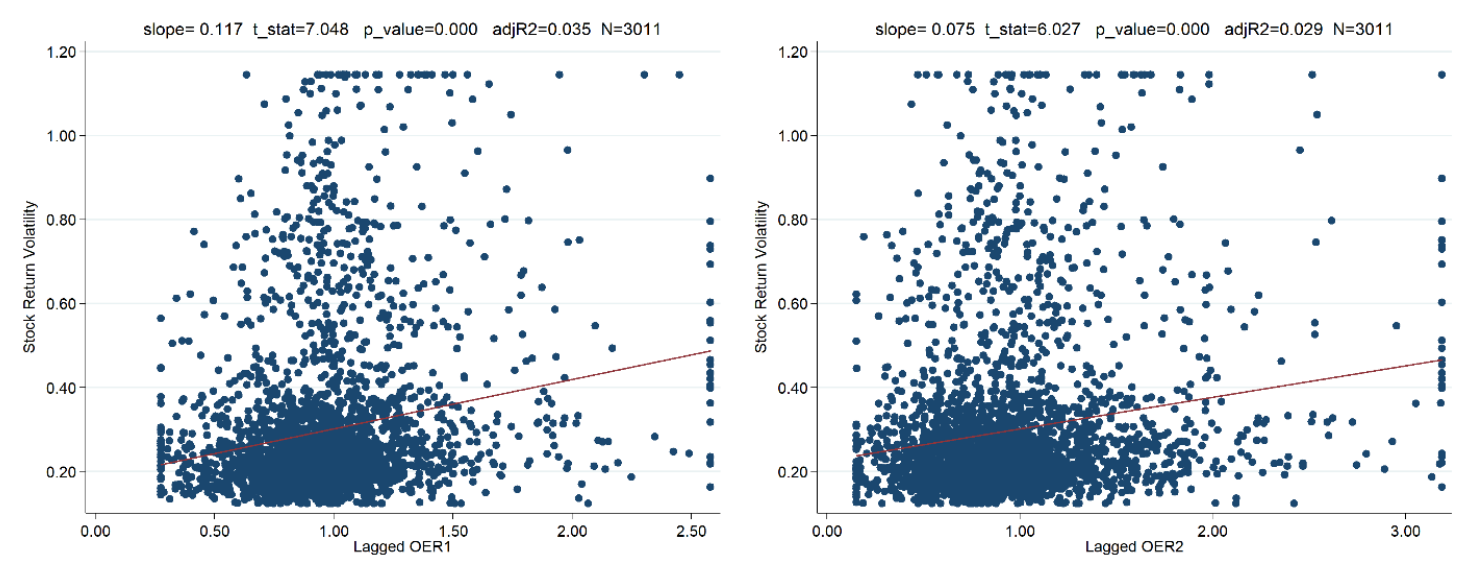

Panel A: Stock Return Volatility and OER
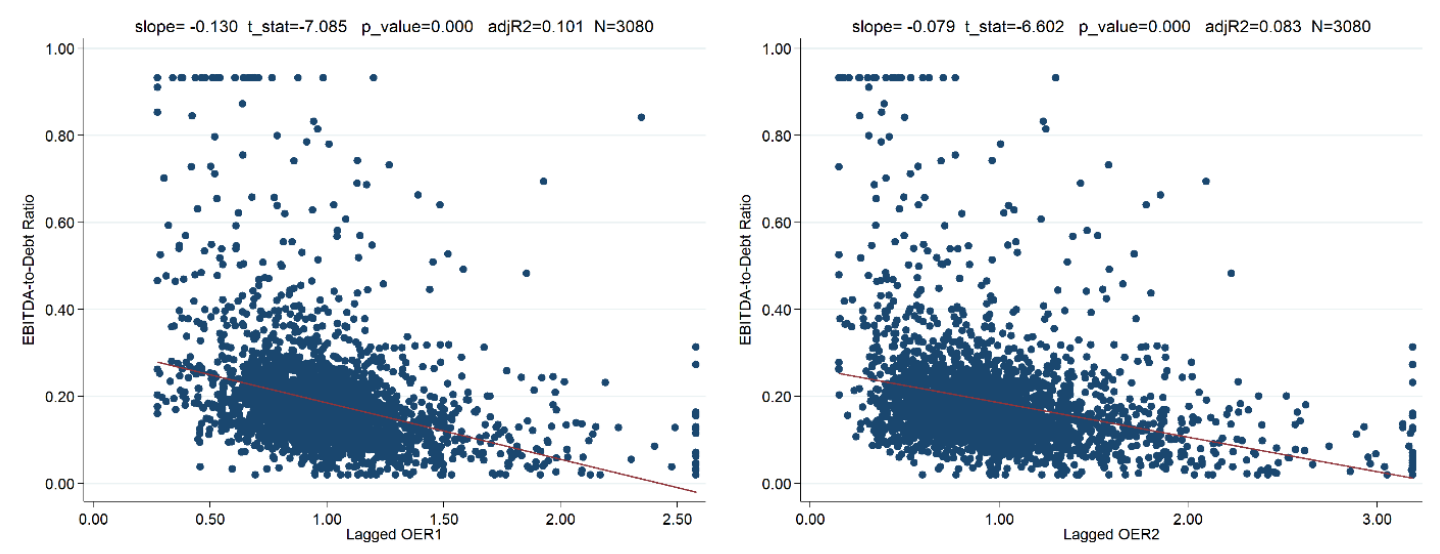

Panel B: EBITDA-to-Debt and OER

Figure 1.2: Firm Risk and Operational Efficiency

This figure plots REIT's total risk, which is measured as its annualized stock return volatility, and Credit Risk, which is measured as EBITDA-to-Debt Ratio, on the vertical axis against two lagged standardized operational efficiency ratios (OERI and OER2, respectively) on the horizontal axis for the sample period $(1995-2016)$. The slope, $t$ statistics, $p$-value and adjusted $R$-squared are reported on the top of each figure. Standard errors are clustered at the firm level and are heteroscedasticity-robust. Significance at the $1 \%, 5 \%$ or $10 \%$ levels is shown with 3,2 , or 1 asterisks, respectively. All variables are defined in Appendix A1. Because the regression specification includes lagged variables, firms with fewer than two consecutive years of stock return and operational efficiency (OERI) information are excluded. Variables have been winsorized at the $1 \%$ and $99 \%$ tails of the distributions to avoid the influence of extreme observations. 

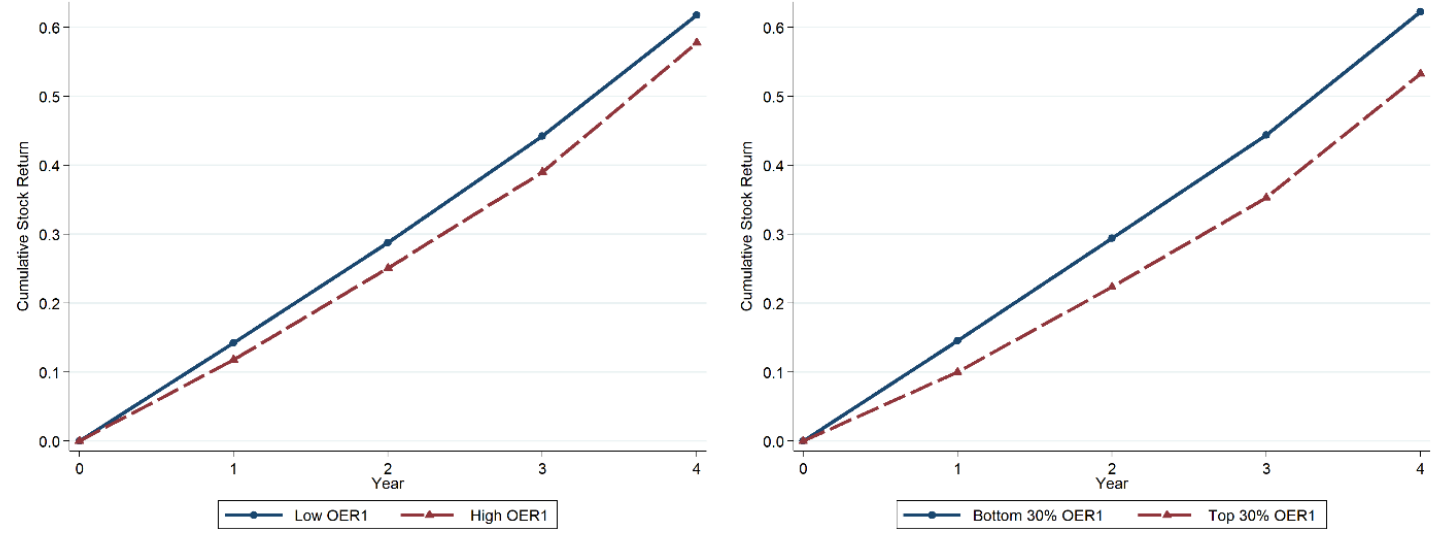

Panel A: Sorted by OER1
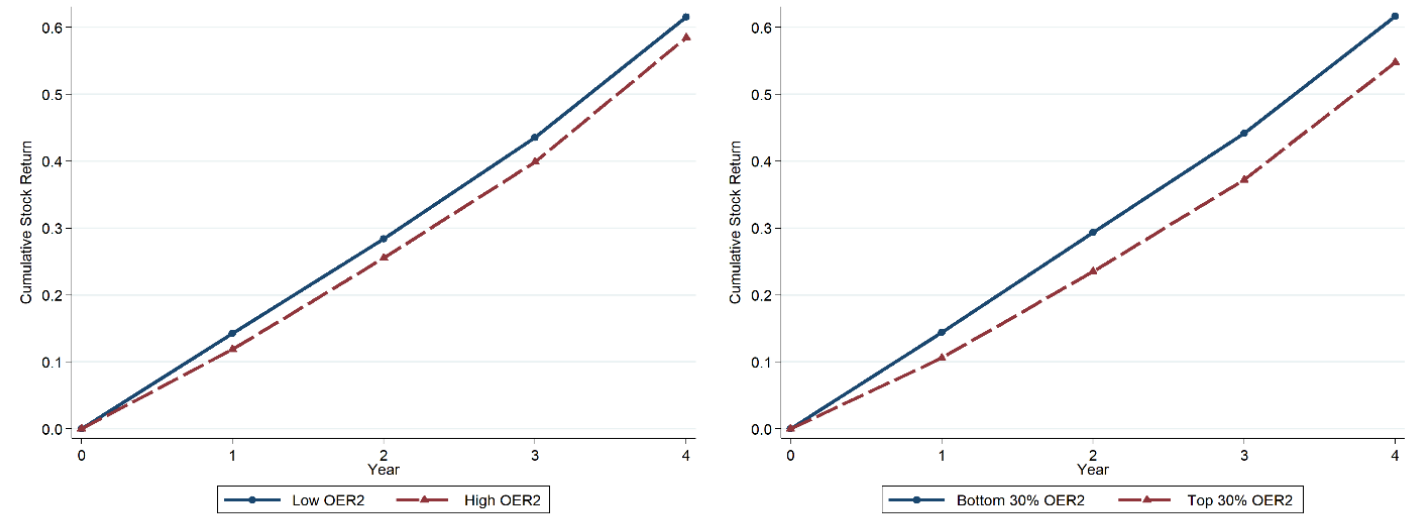

Panel B: Sorted by OER2

Figure 1.3: Cumulative Return of Stock Portfolios Sorted by Standardized OER

This figure illustrates the one- to four- year cumulative return of stock portfolios sorted by standardized operational efficiency ratios (OER1 and OER2). Portfolios are constructed by sorting REITs based on their previous year OER 1 and $O E R 2$. Each year, REITs are divided based on the median (or 30 and 70 percentiles) of $O E R 1$ and OER2, and place REITs with above the median (or 70 percentiles) in the low operational efficiency portfolio and those below the median (or 30 percentiles) in the high operational efficiency portfolio. These portfolios are rebalanced each year. Then, their one- to four- year cumulative return within each portfolio are investigated. All variables are defined in Appendix A1. Because the regression specification includes lagged variables, firms with fewer than two consecutive years of stock return and operational efficiency (OERl) information are excluded. Variables have been winsorized at the $1 \%$ and $99 \%$ tails of the distributions to avoid the influence of extreme observations. 
Appendix

Table A. Definition of Variables

\begin{tabular}{|c|c|c|}
\hline Variable & Abb. & Definition \\
\hline Return on assets & ROA & $\begin{array}{l}\text { Funds from operations divided by lagged } \\
\text { total assets. }\end{array}$ \\
\hline Return on equity & $\mathrm{ROE}$ & $\begin{array}{l}\text { Funds from operations divided by lagged } \\
\text { total equity. }\end{array}$ \\
\hline Stock return & Return & The annualized standard deviation of daily \\
\hline volatility & Volatility & stock return at each firm-year. \\
\hline $\begin{array}{l}\text { EBITDA-to-Debt } \\
\text { ratio }\end{array}$ & $\begin{array}{l}\text { EBITDA-to- } \\
\text { Debt }\end{array}$ & The ratio of EBITDA to total debt \\
\hline Stock Return & Stock Return & $\begin{array}{l}\text { The sum of stock price and dividend paid } \\
\text { divided by lagged stock price, then minus } \\
\text { one. }\end{array}$ \\
\hline Excess stock return & Ret & Stock return minus risk-free rate \\
\hline $\begin{array}{l}\text { Standardized } \\
\text { operational } \\
\text { efficiency ratio one }\end{array}$ & OERl & $\begin{array}{l}\text { The ratio of the ratio of total expense minus } \\
\text { real estate depreciation and amortization to } \\
\text { total revenue to the mean of the ratio of total } \\
\text { expense minus real estate depreciation and } \\
\text { amortization to total revenue of REITs that } \\
\text { have the same real estate property type in } \\
\text { the same year. }\end{array}$ \\
\hline $\begin{array}{l}\text { Standardized } \\
\text { operational } \\
\text { efficiency ratio two }\end{array}$ & OER2 & $\begin{array}{l}\text { The ratio of the ratio of total expense minus } \\
\text { real estate depreciation and amortization } \\
\text { minus rental operating expense to total } \\
\text { revenue minus expense reimbursements to } \\
\text { the mean of the ratio of total expense minus } \\
\text { real estate depreciation and amortization } \\
\text { minus rental operating expense to total } \\
\text { revenue minus expense reimbursements of } \\
\text { REITs that have the same real estate } \\
\text { property type in the same year. }\end{array}$ \\
\hline $\begin{array}{l}\text { Natural log of } \\
\text { Market } \\
\text { capitalization }\end{array}$ & Size & $\begin{array}{l}\text { Market capitalization of common equity, } \\
\text { assuming the conversion of all convertible } \\
\text { subsidiary equity into common. }\end{array}$ \\
\hline Leverage Ratio & Leverage & $\begin{array}{l}\text { The ratio of total book assets to total book } \\
\text { equity. }\end{array}$ \\
\hline Year listed & Firm Age & $\begin{array}{l}\text { The natural logarithm of the number of } \\
\text { years since IPO. }\end{array}$ \\
\hline $\begin{array}{l}\text { Geographic } \\
\text { diversification }\end{array}$ & GeogDiverse & $\begin{array}{l}\text { The negative of the Herfindahl Index of } \\
\text { REITs, calculated using their assets invested } \\
\text { in different MSA location, based on book } \\
\text { values. }\end{array}$ \\
\hline $\begin{array}{l}\text { Property type } \\
\text { diversification }\end{array}$ & $\begin{array}{l}\text { PropTypeDive } \\
\text { rse }\end{array}$ & $\begin{array}{l}\text { The negative of the Herfindahl Index of } \\
\text { REITs, calculated using their assets invested }\end{array}$ \\
\hline
\end{tabular}


in different real estate property type, based on book values.

Firm in S\&P Index Firm in S\&P A dummy variable indicating whether a Index REIT is in S\&P index 


\section{Chapter II: Bank Technology, Performance and Market Value}

\subsection{Introduction}

The past two decades have witnessed the rapid growth in the adoption of innovative technology by U.S. firms. Many believe that, due to the dramatic changes in the technological environment and market structure, firms must actively invest in new technology to provide higher quality products, deliver better customer services, boost revenue and cut costs to stay competitive in the market. Firms that are unable or unwilling to adopt advanced technologies are at a significant disadvantage against their competitors. An important decision faced by managers today is not about whether or not they should embrace advanced technologies, but rather about when to adopt them (Hall and Khan, 2003).

The banking industry has long been one of the most technology-intensive industries in the U.S. (Triplett and Bosworth, 2006). In recent years, technology investment by U.S. banks, including investment in information and financial technologies, has increased at a faster pace even though many of banks have experienced slow revenue growth and strived to cut operating expenses after the financial crisis. ${ }^{1}$ For instance, data on the U.S. listed commercial banks used in this paper indicate that the median technology spending per bank more than doubled since 2000 (see Figure 2-1). Moreover, in 2015, four of the ten biggest

\footnotetext{
${ }^{1}$ See an article from Reuters on September 28, 2016, "Banks Adopting Blockchain 'Dramatically Faster' than Expected: IBM" as well as an article from Financial Times on January 20, 2016, "Big US bank revenue growth is flat as a pancake".
} 
technology spenders worldwide were U.S. banks (Bank of America, Citigroup, JP Morgan Chase \& Co., and Wells Fargo). ${ }^{2}$

With the rapid growth in technology investment over the past two decades, many studies have been conducted to assess the impact of the use of technology in the banking industry. Research suggests that technology progress significantly influences operations, production, and service quality of U.S. banks (Haynes and Thompson, 2000; Berger, 2003; Frame and White, 2014).

Moreover, the adoption of new technology by banks affects market competition, consolidation in the industry, and is likely to create significant regulatory challenges (Berger, 2003; Philippon, 2015). While there is a growing literature on the impact of technological progress and the adoption of new technology, little research has been done regarding the effects of technology investment on U.S. banks at the firm level. ${ }^{3}$

Given the profound impact of technological changes, this paper examines the effects of technology investment on firm performance and market value using a sample of U.S. listed commercial banks. The first research question is to what extent expanded technology investment influences the operating performance of firms. According to Matt Zames, COO of JP Morgan Chase \& Co., technology is "an essential core competency and

\footnotetext{
${ }^{2}$ See a Wall Street Journal article on April 21, 2016, "Wal-Mart Spent \$10.5 Billion on Information Technology in 2015".

${ }^{3}$ A few studies in the literature examine the effects of use of internet on output and performance of U.S. banks. These studies either focus on community banks (DeYoung, Lang, and Nolle, 2007) or use survey data before the "digital network" age (Prasad and Harket, 1997).
} 
a key differentiator to drive future growth" in all of their businesses. ${ }^{4}$ Adoption of new technology helps banks identify new business areas, improve the quality of client services, restructure their business models, improve operational efficiency, and increase competitiveness in the marketplace. ${ }^{5}$ Thus, one would expect technology investment to have a positive long-term impact on bank performance by either increasing revenues and/or improving operational efficiency. However, others contend that technology acts as a double-edged sword and it is often costly to invest in financial technology such as cybersecurity, robo-advising and data analytics, especially for small banks (Dahl, Meyer, and Wiggins, 2017). ${ }^{6}$ Moreover, encroaching automation could wipe out a significant portion of bank profits as fewer fees can be charged for payments such as checks and wires, and revenue from wealth management could drop as well. Thus, the net effect of expanded technology investment on firm performance is ultimately an empirical question.

Also, if the adoption of new technology can improve operational efficiency, provide better service to customers (Melnick, Nayyar, Pinedo, and Seshadri, 2000), and lead to performance gains, one would expect that expanded technology investment should enhance the market value of firms. However, due to managerial entrenchment issues, it is possible

\footnotetext{
${ }^{4}$ See page 52 in the 2015 Annual Report of JPMorgan Chase \& Co.

${ }^{5}$ For example, distributed ledger technology (DLT) was developed to transform payments, clearing, and settlement (PCS) processes (Mills et al., 2016). Also, cloud computing, big data analytics, cyber security, API banking, online and mobile banking, and blockchain enable banks to grow future business. See an article from Business Insider on February 7, 2017, "These are the top trends that will define the banking industry in 2017."

${ }^{6}$ See an article from Wall Street Journal on January 18, 2017, “Technology Will Help-And Hurt-Bank Results, Studies Say."
} 
that managers undertake non-positive net present value (NPV) projects when making technology investment decisions (Fiordelisi and Molyneux, 2010) that could hurt firms in the long run. ${ }^{7}$ Thus, another interesting question is, does expanded technology investment increase or destroy the market value of banks? These two questions are of interest to practitioners, academics, and policymakers as evidenced by the extensive media coverage and industrial reports regarding the use of technology by banks in recent years.

One reason for the limited research regarding the impact of technology investment on operating performance and market value at the firm level is the lack of reliable, comprehensive data about technology investment, as firms are not required to disclose the relevant information to the public. As technology becomes more important to the banking industry, many listed banks have disclosed information on technology spending in their $10 \mathrm{~K}$ reports. S\&P Global Market Intelligence takes a "deep dive" into the banking sector and collects memo items and supplemental financial schedules from U.S. listed commercial banks. The technology spending data include expenses paid for communications, data processing, internet banking, equipment, software purchases and subscriptions to cloudbased services. This unique data set allows us to examine the previously posed two important questions using data on firm-level technology spending as a proxy for technology investment. Table A2 in the appendix provides some examples to detail technology spending data.

\footnotetext{
${ }^{7}$ See Myers and Majluf (1984), Stulz (1990), and Armstrong and Vashishtha (2012), among many others.
} 
Based on a sample of U.S. listed commercial banks from 2000-2017, dramatic growth in technology spending by banks is found. The median technology and communication expense per bank (in 2017 dollars) grew from $\$ 1.12$ million in 2000 to \$2.95 million in 2017 (see Figure 2-1), and it increased almost monotonically over the period. When gross total assets (GTA) is used to group the banks into two subsets, a similar growth pattern is found for both small and large banks. The median technology spending of small banks (large banks) increased from $\$ 0.52$ million ( $\$ 2.57$ million) to $\$ 1.29$ million (\$6.39 million) during the sample period.

An important empirical issue is a potential endogeneity between technology investment and firm performance, as one could argue that firms with better performance are more likely and able to adopt new technology. To investigate this issue, the analysis starts first by taking a closer look at how technology spending is related to bank performance during the recent financial crisis. If technology investment is largely determined by banks' operating performance, one would expect the technology investment to drop significantly over the financial crisis. Interestingly, the results indicate that the median technology spending per bank monotonically grew from \$1.63 million in 2007 to \$1.94 million in 2012 (see Figure 2-1). Besides, when two technology spending ratios (i.e., Tech Expenses/Total Assets and Tech Expenses/Loans \& Deposits) along with the bank performance measures over the sample period are plotted, there is a " $V$ " shape for bank performance measures. However, the median technology spending ratios consistently increased from 2007-2014 (see Figure 2-2). While almost all of the banks experienced a 
negative performance shock and banks strived to cut their expenses during the crisis, the technology spending for small and large banks continued to grow at a steady pace.

Moreover, technology spending measures for those firms experiencing a negative performance shock at the firm-level (see Figure 2-3) are examined. The results indicate that there is no clear pattern for those banks experiencing a negative performance shock in year $t$ to cut their technology spending in the next few years suggesting that a negative performance shock does not seem to affect the technology spending of banks significantly. These findings suggest that expanded technology spending of U.S. banks is less likely to be determined by firm performance.

Next, the results show that the performance measures of banks are positively and significantly correlated with the lagged technology spending measures. Interestingly, the positive correlation is primarily driven by large banks, and there is little evidence concerning the positive correlation based on small banks. The results on small banks are consistent with Hunter and Timme (1986) and Prasad and Harket (1997). A possible interpretation of the results is as follows. Technology investment is often lumpy and costly (e.g., cybersecurity), but banks must adopt new technology to stay competitive.

To some extent, small banks are "forced" to use some of the new technologies even though it may not be "optimal" for them to do so from a pure operational efficiency standpoint. Thus, the financial performance of small banks may not necessarily be improved. In contrast, large banks can better capture the benefits from the adoption of advanced technology due to the economies of scale. 
To examine the channel driving the correlation between firm performance and technology spending, ROA is decomposed into two components: Profit Margin, measuring profitability from sales, and Asset Turnover, measuring sales volume effect. The correlation between the two components and the lagged technology spending measures is investigated. The results indicate that Asset Turnover is positively and significantly associated with the lagged technology spending measures in the three samples (full, small, and large) indicating that technology investment does help increase sales and revenues for both large and small banks. However, in only the large bank sample, Profit Margin is positively and significantly associated with the lagged technology spending measures. These results provide further evidence that the use of technology does not necessarily improve the performance of small banks. Thus, technology investment is likely to affect firm performance by increasing sales volume and revenue, rather than by improving operational efficiency. Moreover, large banks benefit from expanded technology investment through efficiency gains and cost reductions resulting from the economies of scale.

Furthermore, additional robustness checks indicate that the positive correlation between firm performance and lagged technology spending measures still hold when toobig-to-fail banks are excluded, when banks with mergers and acquisition activities are excluded, and when banks with a relatively small amount of technology spending are excluded. More importantly, when long lags of the technology spending measures are used, the results indicate that the positive correlation holds for the large bank group up to four lags. 
Taken together, these findings suggest that the expanded technology spending by banks is more of a necessity, instead of a strategic choice, and technology investment helps to improve the financial performance of large banks. It is worth noting that despite the attempts to investigate the endogeneity issue between bank performance and technology spending, it remains an empirical issue for future research due to the data limitations researchers face.

Regarding the effect of technology investment on firm value, bank market value, measured as the market-to-book equity ratio and $Q$, is positively and significantly associated with their lagged technology spending measures for large banks. However, there is little evidence regarding the correlation between bank market value and the lagged technology spending measures for small banks. This finding suggests that shareholders of large banks do recognize the benefits of expanded technology spending.

Overall, the results in the paper suggest that investment in technology particularly benefits large banks by improving their financial performance and increasing firm value. However, there is little evidence that financial performance and the market value of small banks are significantly improved by expanded technology spending. These findings provide insight for managers to make efficient capital allocation decisions and have important implications for policymakers to modify regulations regarding the use of information and financial technologies.

This paper makes the following contributions to the literature. First, this paper is one of the first studies examining the extent to which technology investment influences 
firm performance and market value using firm-level technology spending data of U.S. listed banks. Previous studies have largely focused on the impact of technology innovations (proxied by the number of patents) or research and development (R\&D) expenses on corporate decisions and valuation. Thus, this paper provides a different perspective to examine the impact of technology on firms, and the findings suggest that firms are more likely to benefit from expanded technology investment through increase in sales volume rather than efficiency gains.

Second, this paper helps to explain the "IT performance paradox" in the literature (e.g., Haynes and Thompson, 2000; Mithas, Tafti, Bardhan, and Goh, 2012). Many studies based on data from manufacturing firms indicates that there is no relationship between technology investment and firm performance, while others find a strong positive correlation. There is a firm size effect. While there is no significant correlation between firm performance and technology investment for small banks, a positive correlation exists for large banks. It implies that use of technology is more of a necessity for small firms in order to stay competitive in the market. As it is often expensive to adopt advanced technology, small firms may not benefit from technology investment the same as large firms.

Finally, this paper examines the relationship between technology spending and firm value. The findings suggest that the market does value technology investment by large banks, perhaps because technology can improve the quality of client service, create new lines of business, and improve operating efficiency. Thus, this paper fills a gap in the literature as to whether technology investment enhances or destroys the firm value. 


\subsection{Background and Literature}

In banking literature, following the seminal work by Sealey and Lindley (1977), many studies examine the impact of technology changes and progress on bank production and services, as well as the market structure of the banking industry. Hunter and Timme (1986) investigate the impact of technical changes on bank production and scale economies. Using bank holding company data collected from the Bank Compustat file from 1972-1982, they find that technical changes exhibit positive scale bias. In other words, large banks are more likely to fully exploit operating scale economies and remain competitive in deposit markets based on their operating efficiencies resulting from the use of new technology.

Petersen and Rajan (2002) examine the distance between small firms and their lenders based on a sample obtained from the 1993 National Survey of Small Business Finance (NSSBF). Their findings suggest that information technology provides greater information availability to small firms and reduces the costs of processing small business loans that contribute to a longer distance between small firms and their lenders. They conclude that there is indirect evidence that information technology does increase bank productivity.

Berger (2003) examines technological progress and its effects on productivity growth and the market structure of the banking industry. Based on banking data from various sources from 1984-2001, their study suggests that technology (primarily Internet banking, electronic payment technology, and information exchanges) significantly improve the quality of banking services and increase bank productivity. Specifically, 
consumers benefit from improved "front-office" technology (those directly dealing with customers) and "back-office" technology (those invisible to customers) that help to reduce costs and improve lending capacity. Moreover, the paper suggests that technological progress has a significant impact on the market structure of U.S. banks and helps facilitate banking consolidation. This view is also supported by Pang (2018) who proposes a theoretical model to identify the winners and losers of the advances in bank information technology.

Based on various proxies for the use of technology, a few studies investigate the different effects of technology on bank investment. For example, Saloner and Shepard (1995) find that the adoption of automated teller machines (ATMs) delays the decline in the number of branches for banks. Ferrari, Verboven, and Degryse (2010) examine the investment and demand of ATMs and determine that banks substantially underinvest in ATMs.

Using either survey data, data from small community banks, or data from European banks, a few studies examine the effects of technology on the financial performance of banks. An early study by Prasad and Harket (1997) examines the contributions of information technology (IT) on profitability in U.S. retail banking. Based on a survey dataset of U.S. retail banking institutions from 1993-1995, they find that an increase in IT investment does not benefit banks' productivity and financial performance. They argue, since there is no "barrier to entry" in terms of IT in the retail banking industry, small retail banks must adopt new technology in order to stay in the competition, even though it is not 
in the best interest of them to use the technology based on cost-benefit considerations. Thus, the use of IT may not necessarily have a positive relationship with bank performance.

A more recent study by DeYoung, Lang, and Noelle (2007) employs U.S. community bank data from 1999-2001 to study the impact of transactional banking websites on bank performance. They determine that internet adoption improves performance for community banks, primarily through increased revenues from deposit service charges. In contrast, Arnaboldi and Claeys (2010) find little evidence of economies of scope on the use of technology by European banks. They confirm that there is little gain from internet banking investment based on a panel of the 60 largest European banking groups from 1995-2005. Moreover, based on a sample of 737 European banks from 19952000, Beccalli (2007) investigates whether IT investment improves bank performance. The paper also finds little relationship between IT investment and bank performance or efficiency. However, their research demonstrates that investments related to IT service from external providers (e.g., consulting services, training, and education) have a positive impact on financial performance, while investments in hardware and software are negatively related to banks' profits. In short, the results from these papers suggest that there is mixed evidence about the effect of the use of technology on bank performance.

Also, Martin-Oliver and Salas-Fumas (2008) and Martin-Oliver, Ruano, and SalasFumas (2013) use data from Spanish commercial banks to examine the impact of technology investment before the financial crisis. They find some evidence that technology investment influences the productivity and performance of Spanish banks. Sullivan and Wang (2013) study the endogenous diffusion and impact of Internet banking, which is 
believed to be a cost-saving technological innovation. They suggest that large banks could take advantage of being early adopters and could increase in size at the time when the innovation was initially introduced. While the focus is to assess the impact of technology on the financial industry in terms of financial stability and access to services, Philippon (2015) provides some evidence that the adoption of financial technology does not reduce the intermediation costs for banks.

There are also studies that examine the economic benefits of technology adoption using data from manufacturing firms. The results are mixed and inconclusive. Berndt and Morrison (1995) find limited evidence of a positive relationship between firm performance and their ratios of high-tech capital to physical capital stock in U.S. manufacturing industries from 1968-1986. Hitt and Brynjolfsson (1996) confirm the little relationship between firms' use of information technology and their performance. More recently, a positive and statistically significant technology and performance relationship is noted in Mithas et al. (2012) whose sample is more than 400 large global firms. They also find that IT-enabled revenue growth, but not operating cost reduction, significantly contributes to this positive relationship. ${ }^{8}$

Most of the studies in the banking literature focus on the effects of technology on bank productivity, quality of services, and the market structure. While there are a few papers that examine the relationship between technology investment and bank performance,

\footnotetext{
${ }^{8}$ Other studies in the literature include Brynjolfsson (1993). A detailed review of the literature can be found in Brynjolfsson and Yang (1996) and Draca, Sadun, and Van Reenen (2007).
} 
the extant research is largely based on limited survey data from U.S. banks or short-term data prior to the "network" era of computing that cannot evaluate the effects of technology investment in the banking industry during the past decade.

For studies based on data from European banks, one can argue that significant differences exist between the U.S. banking system and the European banking system (e.g., market structure and regulations, sample period, and size of the industry). ${ }^{9}$ Thus, the results based on European bank data may not apply to the U.S. banking industry. Also, previous literature largely uses proxies for technology usage, such as the number of ATMs and transaction website adoption for information technology. There is little research based on direct firm-level technology spending data from U.S. listed banks. More importantly, the results regarding financial performance and technology spending are largely mixed in the literature, suggesting that further research on this issue is warranted.

Using technology spending data from Fortune 1000 companies around the year 2000 (Y2K), Anderson, Banker, and Ravindran (2006) find technology investment increases firm value. Yet, there is little research in the banking literature as to how technology investment influences the firm value of banks, while the impact of other factors on bank value have been widely examined including bank deregulation (Marcus, 1984), diversification (Elsas, Hackethal, and Holzhäuser, 2010), and equity capital (Mehran and Thakor, 2011). If banks can provide better customer service via technology (Melnick et al.,

\footnotetext{
${ }^{9}$ The GDP of Spain in 2015 is roughly $6.6 \%$ of the GDP of the U.S. The total assets of Spanish banks in 2016 is about $€ 2.7$ trillion based on a BBVA research report and the total assets of U.S. commercial banks at the same year is $\$ 12$ trillion based on FRED economic data.
} 
2000) and bank performance is positively related to their technology spending, the market value of banks should also be positively correlated with their technology spending. Meanwhile, not all technology investments are placed on positive NPV projects as bank managers may have potential managerial entrenchment issues (Fiordelisi and Molyneux, 2010). Thus, the relationship between bank value and technology investment becomes an empirical question.

The banking literature also notes significant differences in portfolio composition and technology adoption strategies for small and large banks (Berger and Udell, 2002; Berger and DeYoung, 2006; Berger and Bouwman, 2013). In addition, it is well known that there exist economies of scale in technology investment (Katz and Shapiro, 1986; Harris and Katz, 1991; Hall and Khan, 2003), which implies that technology investment could have different effects on firms with different size. Thus, it is meaningful to examine the impact of technology spending on both large and small banks.

To what extent is the use of technology exogenously determined? Previous research suggests that technology has profoundly changed the way that traditional business is conducted and technological advances have become an exogenous driving force in the economy (Greenwood, Hercowitz, and Krusell, 1997; Hansen and Prescott, 2002). ${ }^{10}$ Additionally, the innovation and adoption of technology from the world technology

\footnotetext{
${ }^{10}$ Technology is often assumed as exogenous in the neoclassical growth model (Grossman and Helpman, 1994) and in the real business-cycle theory (Kydland and Prescott, 1982; Greenwood, Hercowitz, and Huffman, 1988; Gali, 1999).
} 
frontier of firms drives the economic growth (Acemoglu, Aghion, and Zilibotti, 2006; Madsen, 2014).

With the rapid technological progress across the banking industry, many believe that banks must adopt new technology in order to stay competitive, while others argue that adoption of technology may be driven by certain firm characteristics. Hall and Khan (2003) contend that the choice of technology adoption is between adopting it now or deferring the decision until later, but it is not a choice between adopting and not adopting. To stay competitive and better serve customers, banks should invest or adopt new technology at some point in time. Hernândez-Murillo, Llobet, and Fuentes (2010) investigate the determinants of banks' decisions to adopt a transactional website for their customers. Using a panel of commercial banks in the U.S. from 2003-2006, they find that while bank-specific characteristics are important in banks' adoption decisions, market competition plays a prominent role consistent with the notion that the adoption of technology is a necessity for banks to remain competitive. Overall, previous research seems to support that the use of technology by banks can be exogenously determined.

\subsection{Data and Research Methodology}

\subsubsection{Data Description}

The firm-level data set for this study is obtained from the Compustat banking database, and the S\&P Global Market Intelligence (formally SNL Financial) banking database from 2000-2017 for the U.S. listed commercial banks (SIC Code: 60). Specifically, 
the annual technology and communication expense data are collected from the S\&P Global Market Intelligence. Other annual financial information is obtained from Compustat. ${ }^{11}$

Technology and communication expenses include physical technology equipment, software, and services. However, it is challenging to disentangle the components of this expense from the database. The technology expense from the S\&P Global Market Intelligence is primarily constructed based on U.S. GAAP Standard Statement of Financial Accounting Standards (FAS) No. 86 and includes expenses paid for communications, such as telephone and fax usage charges, internet data plans, internet plans, data processing, technology equipment as well as software purchases and subscriptions to cloud-based services. On the financial reports and bank regulatory filings of banks, these expenses are usually reported as technology and communications expenses, data processing expenses, Internet banking expenses, and ATM expenses, etc. Table A2 in the appendix provides detailed descriptions of the technology expenses, along with some typical examples indicating their original sources and compositions.

Regarding performance measures of banks, two commonly used ratios are used: Return on Assets (ROA) and Return on Equity (ROE), which are defined as earnings before extraordinary items plus depreciation and amortization divided by the book value of assets and by the book value of equity, respectively. To assess which channel of technology spending affects bank performance, ROA is decomposed into two components: Profit

\footnotetext{
${ }^{11}$ If an accounting item is missing in year $t$, it is replaced by estimates from this formula: $\operatorname{In} f o_{i, t}^{x}=$ $\left(\operatorname{Inf} o_{i, t+1}^{x}+\operatorname{Inf} o_{i, t-1}^{x}\right) / 2$, where $\operatorname{Inf} o_{i, t}^{x}$ is the information of $x$ (total assets, technology expense, etc.) of bank $i$ in year $t$.
} 
Margin and Asset Turnover. Profit Margin is measured as earnings before extraordinary items plus depreciation and amortization divided by the sum of interest income and noninterest income, while Asset Turnover is measured as the sum of interest income and noninterest income divided by the book value of assets. The market value of banks is measured as the market-to-book equity ratio (market-to-book), defined as share prices as of the fiscal year-end times common shares outstanding divided by the book value of equity, and $Q$ as in Lamont and Polk (2002), measured as share prices as of the fiscal year-end times common shares outstanding plus total assets minus the book value of equity, to total assets. Table A1 in the appendix provides detailed descriptions of the variables used in this paper.

As large banks may spend more on technology, it needs to adjust for firm size making it meaningful to compare technology spending among banks. Thus, controlling for firm size, two technology ratios (technology expense/total assets and technology expense/total loan and deposit) are included as independent variables in the regressions. ${ }^{12}$

Since lagged variables are included in the regressions, banks with fewer than two consecutive years of technology expenses and total assets information are excluded. Observations with missing values of the key variables are also excluded. All the variables

\footnotetext{
${ }^{12}$ It is possible that the technology expense variable does not include all technology investment. By normalizing the technology expense variable using firm size and including bank fixed effects in the regression, the measurement error problem is mitigated. It also alleviates the problem resulting from an increase in bank size, especially from mergers and acquisitions.
} 
are winsorized at the $1 \%$ and $99 \%$ tails of the distributions to mitigate the effect of outliers. The final sample consists of 8,706 bank-year observations for 994 banks from 2000-2017.

Table 2-1 reports the summary statistics of the key variables used in this paper. The total assets of the banks have a mean of $\$ 7.1$ billion and a median of $\$ 0.9$ billion. The typical bank has an average market capitalization of $\$ 1.1$ billion and a median of $\$ 0.1$ billion. The mean and median of the technology expenses is $\$ 14.3$ million and $\$ 1.5$ million, respectively. Regarding the performance measures, the mean Return on Assets is $0.82 \%$ and the median is $0.94 \%$, while the mean Return on Equity is $8.28 \%$ and the median is $9.77 \%$. For measures of firm value, the average market-to-book equity ratio is 1.33 and the median is 1.21 , and the average $Q$ is 1.03 and the median $Q$ is 1.02 . Regarding the technology expense measures, the mean (median) ratio of technology expense over total assets and total loans and deposits are $0.18 \%(0.17 \%)$ and $0.13 \%(0.12 \%)$, respectively.

2.3.2. Empirical Specifications for the Correlation between Performance and Technology Spending

This subsection discusses the empirical specification to assess the relationship between bank performance and technology spending. Return on Assets (ROA) and Return on Equity (ROE) are used as the performance measures. ROA demonstrates how well a firm can generate a return on its assets and indicates whether a bank's assets are productive and well managed. ROE is the measure of company performance in the views of analysts and investors. The relations between the ROA and ROE of banks and their previous year's 
technology spending measures, controlling for several firm characteristics based on the full sample, small bank, and large bank sample, respectively, are examined. A fixed effects model is used with heteroskedasticity-robust standard errors clustered at the firm level as follows:

$$
\begin{aligned}
& \operatorname{Perf}_{i, t}=\beta_{0}+\beta_{1} \text { Tech }_{i, t-1}+\beta_{2} \text { LnSize }_{i, t-1}+\beta_{3} \text { Leverage }_{i, t-1}
\end{aligned}
$$

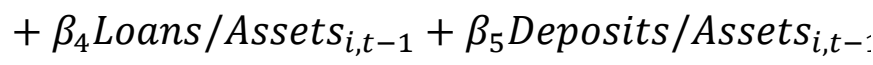

$$
\begin{aligned}
& +\beta_{6} \text { Deposits }_{\text {Liability }_{i, t-1}}+\eta_{i}+\alpha_{t}+\varepsilon_{t}
\end{aligned}
$$

where $P \operatorname{erf} f_{i, t}$ is either the ROA or ROE of bank $i$ at year $t, \operatorname{Tech}_{i, t-1}$ is either the ratio of technology expense over total assets or the ratio of technology expense over total loans and deposits of bank $i$ at year $t-1$. LnSize $_{i, t-1}$ is the previous year's natural log of the market capitalization of equity following Cheng, Hong, and Scheinkman (2015). Leverage $e_{i, t-1}$ is the previous year's book assets to equity ratio, as in Adrian and Shin (2009). Loans / Assets $_{i, t-1}$ is the previous year's total loans to total assets ratio. Deposits / Assets i,t-1 $_{\text {is }}$ is the previous year's total deposits to total assets ratio, Deposits / Liability $_{i, t-1}$ is the previous year's total deposits to total liabilities ratio, and $\eta_{i}$ and $\alpha_{t}$ represent firm and year fixed effects, respectively.

ROA can be expressed as the product of Profit Margin and Asset Turnover. This decomposition is widely used in the literature (Muscarella and Vetsuypens, 1990; Jansen, Ramnath, and Yohn, 2012) and can be found in financial statement analysis textbooks (e.g., Healy and Palepu, 2012, Titman, Keown, and Martin, 2013; Brigham and Daves, 2014). 
Intuitively, Profit Margin indicates how much of every dollar of revenue a bank keeps in earnings. It reflects a bank's operating efficiency and profitability after considering the costs of goods and/or services sold and operating expenses. Asset Turnover measures asset utilization indicating how well a company can deploy its assets in generating revenue. Some firms may emphasize high Profit Margin to improve their ROA, while others pay closer attention to Asset Turnover or both. The existing literature provides little evidence regarding the relationship between bank technology investments and Profit Margin and/or Asset Turnover.

To examine the specific channel that technology spending influences bank performance, the following equation to study the relationship between the two components and technology spending is posited:

$$
\begin{aligned}
& \text { ROA Comp }_{i, t}=\beta_{0}+\beta_{1} \text { Tech }_{i, t-1}+\beta_{2} \text { LnSize }_{i, t-1} \\
& +\beta_{3} \text { Leverage }_{i, t-1}+\beta_{4} \text { Loans }_{/ \text {Asset }_{i, t-1}} \\
& +\beta_{5}{\text { Deposits } / \text { Assets }_{i, t-1}} \\
& +\beta_{6} \text { Deposits }_{\text {Liability }} \text { Lit }-1_{1}+\eta_{i}+\alpha_{t}+\varepsilon_{t}
\end{aligned}
$$

The dependent variable, ROA $\operatorname{Comp}_{i, t}$, is either ProfitMargin ${ }_{i, t}$ (defined as earnings before extraordinary items plus depreciation and amortization divided by the sum of interest income and noninterest income) or Asset Turnover $_{i, t}$ (defined as the sum of interest income and noninterest income divided by the book value of assets) of bank $i$ at year $t$. Other variables are as previously defined. 


\subsubsection{Firm Value and Technology}

This subsection discusses the methodology to examine the relationship between technology investment and firm value. In value-based management (VBM) theory, the primary objective of a firm is to increase the wealth of its shareholders (Ittner and Larcker, 2001). Firm managers should first consider the interests of shareholders when making investment decisions. Thus, sound technology investment decisions should help maximize firm value. In addition, if technology investment influences the financial performance of banks, there should exist a positive relationship between firm value and technology investment.

To empirically examine the relationship, a similar regression as follows is adopted.

$$
\begin{aligned}
& \text { FirmValue }_{i, t}=\beta_{0}+\beta_{1} \text { Tech }_{i, t-1}+\beta_{2} \text { LnSize }_{i, t-1} \\
& +\beta_{3} \text { Leverage }_{i, t-1}+\beta_{4} \text { Loans }_{\text {Assets }}, t-1 \\
& +\beta_{5} \text { Deposits } / \text { Assets }_{i, t-1}
\end{aligned}
$$

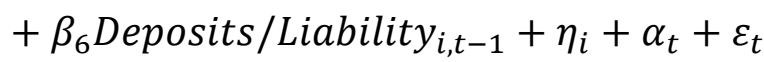

The dependent variable is the market-to-book equity ratio or the $Q$ of bank $i$ at year $t$ and the other variables are as previously defined. Here, the market-to-book equity ratio is defined as share prices (common shares outstanding at the fiscal year-end) divided by the book value of equity. $Q$ is the ratio of the market value, measured as share prices as of 
the fiscal year-end times common shares outstanding plus total assets minus the book value of equity, to its total assets as in Lamont and Polk (2002).

\subsection{Empirical Results}

2.4.1. The Growth of Technology Spending and Its Relationship with Firm Performance

First, dramatic growth in technology spending by banks from 2000-2017 is found. The median technology and communication expense per bank (in 2017 dollars) grew from $\$ 1.12$ million in 2000 to $\$ 2.95$ million in 2017 (see Figure 2-1). The trend is almost monotonically increasing during this period. Then, the banks are grouped into two subsets based on their gross total assets (GTA). For small banks, whose time-series average of GTA is up to $\$ 1$ billion, the median rose from $\$ 0.52$ million to $\$ 1.29$ million, while for large banks, whose time-series average of GTA exceeds $\$ 1$ billion, the median grew from $\$ 2.57$ million to $\$ 6.39$ million. This finding indicates that there exists a steady increase in a bank's technology spending each year over this period. ${ }^{13}$ The result is also in line with the final sales of domestic computers in the United States, as shown in Table 9.2U of the National Income and Product Accounts and illustrated in Figure B1 in the appendix. ${ }^{14}$

\footnotetext{
${ }^{13}$ U.S listed commercial banks without mergers and acquisitions activities (Non-M\&A banks) are kept in the sample from 2000-2017. There are 538 Non-M\&A banks in the sample. The trends of technology and communication expenses (based on median) of those Non-M\&A banks are illustrated. The results are quantitatively similar and are reported in Figure B1 in the appendix.

14

https://apps.bea.gov/iTable/iTable.cfm?ReqID=19\&step=3\#reqid=19\&step=3\&isuri=1\&1921=underlying\&1903=2076
} 
To assess whether banks with better financial performance are more likely to adopt new technology, a closer look is taken at how technology spending is related to bank performance during the financial crisis. The argument is that if technology spending is largely determined by a bank's financial performance, then technology spending should have dropped significantly during the financial crisis. The median technology spending per bank grew from $\$ 1.63$ million in 2007 to $\$ 1.94$ million in 2012 (see Figure 2-1), although the median bank performance measures experienced a significant drop starting from 2007. Also, the median technology spending measures (Tech Expenses/Total Assets and Tech Expenses/Loans \& Deposits) gradually increased from 2007 until 2014 (see Figure 2-2), while there is a clear "V" shape for bank performance measures over the sample period.

Next, if bank performance strongly influences technology investment, one would expect technology spending to decrease when banks face significant financial hardships. Essentially, it is examined how technology spending reacts on a negative performance shock, which is measured as a bank's ROA becomes negative at year $t$, while it is positive in year $t-1$. In the sample, there are 247 bank years that a bank's ROA becomes negative from a positive previous year. The technology spending trend three years before and after the negative performance shock year are examined. ${ }^{15}$

\footnotetext{
${ }^{15}$ The negative performance shock measure is also measured as a bank's ROA at year $t$ is one standard deviation less than that of year $t-1$. In the sample, there are 258 bank years that a bank's ROA drops two standard deviations from its previous year. The results are quantitatively similar and are reported in Figure B2 in the appendix.
} 
Figure 2-3 illustrates the pattern of the mean and the median of the technology spending measures. The results indicate that the mean and median technology spending measures (Tech Expenses/Total Assets and Tech Expenses/Total Loan \& Deposits, respectively) after the year with a negative performance shock still increase monotonically in both cases. However, the mean (median) Tech Expenses/Total Assets ratio in year $t+3$ is higher than the ratio in year $t-3$ by $0.04(0.03)$, with a $t$-statistic from the two sample $t$ test of 5.17 and a $z$-statistic from the two sample Wilcoxon rank-sum test of 4.94. The test results based on Tech Expenses/Total Loan \& Deposits are similar. These results indicate that technology spending continues to increase even when banks experience a negative performance shock, suggesting that a negative shock on bank performance does not affect their technology spending. ${ }^{16}$

\subsubsection{Firm Performance and Technology Spending}

This section explores the correlation between bank performance, measured by Return on Assets (ROA) and Return on Equity (ROE), and their lagged technology spending measures, measured by Tech Expenses/Total Assets and Tech Expenses/Loans \& Deposits, while controlling for firm size, capital structure, and portfolio composition in the previous year.

\footnotetext{
${ }^{16}$ Same analysis is also applied by measuring bank negative profitability shocks via ROE. The results are quantitatively similar with those presented in this panel. For brevity, these results are not reported, but are available upon request.
} 
Table 2-2 reports the regression results based on Equation (2-1) (i.e., the basic regression of bank performance on the lagged technology investment measures), when ROA is used as the dependent variable. In Columns (1) and (2), when, the estimated coefficients of the previous year's technology spending measures are positive $(0.60$ and 0.85) and statistically significant at the $10 \%$ level. For small banks [Columns (3) and (4)], the estimated coefficients of the previous year's technology spending measures are statistically insignificant. In contrast, the coefficients of the previous year's technology spending measures based on large banks are all positive (0.93 and 1.32) and highly statistically significant at the $1 \%$ level, as in Columns (5) and (6). It is worth noting that the coefficients of the large banks are significantly greater than those of the full sample and the small banks. The result highlights the importance of technology investment on performance for large banks. For the control variables, the results confirm that bank size is positively correlated with ROA indicating that large banks tend to have better performance. Moreover, firm leverage is negatively associated with ROA, which is not surprising.

Similarly, with ROE as the dependent variable Table 2-3, the coefficients are also positive (10.59 and 15.14) and statistically significant at the 5\% level for the full sample [Columns (1) and (2)]. For small banks sample [Columns (3) and (4)] the estimated coefficients of the previous year's technology spending measures are positive, but statistically insignificant. In the meanwhile, the coefficients of the previous year's technology spending measures based on large banks [Columns (5) and (6)] are positive (15.75 and 22.12) and highly statistically significant at the $1 \%$ level. The results also show that bank size is positively correlated with performance. As expected, banks with a higher 
deposit/assets ratio tend to have higher ROE, while those with a higher deposit/total liabilities ratio tend to have lower ROE.

The insignificant coefficients of the technology spending measures for small banks can partly explain the "IT performance paradox" stated in Beccalli (2007) whose sample consists of commercial banks from five European countries. The positive relationship between technology spending and performance for large banks is consistent with Mithas et al. (2012), a recent study whose sample contains more than 400 large global firms. Collectively, the results provide strong evidence that bank performance is positively related to their previous year's technology spending for large banks, but not small banks.

\subsubsection{Channel of Technology Spending Influencing Firm Performance}

The results presented in this subsection shed light on the mechanism in which a bank's performance is associated with its technology spending. As mentioned previously, a bank's Return on Assets are decomposed into two parts: Profit Margin, measuring operating efficiency and profitability, and Asset Turnover, measuring sales volume effect.

When the dependent variable is Profit Margin, the results from Equation (2-2) are reported in Table 2-4. With the full sample, as in Columns (1) and (2), the estimated coefficients of the previous year's technology spending measures are positive, but statistically insignificant. For small banks, the estimated coefficients of the previous year's technology spending measures are negative and statistically insignificant, as in Columns 
(3) and (4). For the large banks, the estimated coefficients of the previous year's technology spending measures are positive (14.57 and 19.40) and statistically significant at the 5\% or $10 \%$ level, as Columns (5) and (6).

While the dependent variable is Asset Turnover, the results from Equation (2-2) are reported in Table 2-5. All six estimated coefficients of the previous year's technology spending measures are positive [1.88 and 2.78 for the full sample as in Columns (1) and (2), 1.64 and 2.45 for the small banks sample as in Columns (3) and (4), and 1.97 and 2.89 for the large banks sample as in Columns (5) and (6)] and statistically significant at the 1\% level. The results imply that banks with higher previous year's technology spending, on average, generate more revenues.

The results imply that technology spending does help small banks to increase their revenue (higher Asset Turnover) but does not increase their ability to convert revenue into profit. Thus, the revenue generated from the technology investment of small banks may not fully cover their increased costs. Large banks can better capture the benefits from technology investment through efficiency gains and cost reduction resulting from the economies of scale, which suggests that bank size matters in such a relation. These results from the full sample also suggest that Asset Turnover, but not Profit Margin, may be associated with their previous year's technology spending. These results imply that technology investment is likely to affect bank performance by increasing sales volume rather than by improving operational efficiency. 


\subsubsection{Market Value and Technology Investment}

This subsection examines the extent to which the technology investment of a bank is a source of value creation for its shareholders. The results from Equation (2-3) with the market-to-book ratio as the dependent variable are reported in Table 2-6. The coefficient estimates of the technology spending measures are statistically insignificant in the full sample and the small bank sample, as in Columns (1) to (4). In contrast, the coefficient estimates of the technology spending measures for the large banks are positive $(0.53$ and 0.66) and statistically significant at the 5\% level, as in Columns (5) and (6).

The results from Equation (2-3) with Firm $Q$ as the dependent variable are reported in Table 2-7. Consistently, the four coefficient estimates of the previous year's technology spending measures are statistically insignificant in the full sample and the small banks sample, as in Columns (1) to (4), while they are positive (0.04 and 0.05) and statistically significant at the $10 \%$ level, in the large bank sample, as in Column (5) and (6).

Collectively, these results provide evidence that bank value is positively associated with their previous year's technology spending related to total assets and total loans and deposits, respectively, in large banks. The results are consistent with Anderson et al. (2006) who focus on Fortune 1000 firms and find that firm value increased, on average, with Y2K spending on technology. In addition, the estimated coefficients of the technology spending measures in the large bank sample are greater than those in the full sample and small bank sample. 


\subsection{Robustness Checks}

This section presents a range of robustness checks, which provide additional supporting evidence for the main results in Section 4. The control variables used in this section are the same as those in the main regression models unless noted below.

\subsubsection{Alternative Technology Spending Levels}

As argued in the previous section, some banks seem to benefit significantly from their technology investments only if the amount of their technology spending is large enough and can change their business model and significantly lower their operating cost

due to the economies of scale. Other banks may not be able to capture the benefits of technology investments as technology investments are often lumpy and costly. The analysis is re-run using $\$ 1$ million and $\$ 3$ million as the cutoff point for technology expense, respectively.

Panels A and B of Table 2-8 report the results of the regressions of the performance and firm value measures on technology spending measures with the three cutoff points. The parameters for the lagged technology spending measures in the $\$ 5$ million sample and the \$3 million sample are larger and have higher statistical significance levels than those in the \$1 million sample. These results support the main results in Section 4. 


\subsubsection{Large Banks with Too-Big-To-Fail (TBTF) Banks Excluded}

It is believed that the largest banks operate in very different models and under different degrees of government regulation, supervision, and support. To ensure the results concerning large banks are not determined by those TBTF banks, an analysis for the large bank sample excluding banks whose time-series average gross total assets exceed $\$ 50$ billion is conducted. Those banks are usually called too-big-to-fail (TBTF) banks or systematically important financial institutions (SIFIs).

Panel D of Table 2-8 reports the regression results on Return on Assets, Return on Equity, Profit Margin, Asset Turnover, market-to-book, and $Q$ of the banks on their previous year's technology spending measures with each column as a separate regression for the dependent variable indicated in the column header. The estimated coefficients are quantitatively similar to those presented in the previous tables and significance is found in similar cases except for $Q$. The findings of the relationship between the performance and firm value of large banks and their technology investment measures remains unchanged when TBTF banks are dropped from the sample.

\subsubsection{Large Banks without Mergers and Acquisitions Activities}

One may concern that consolidation or mergers and acquisitions activities may drive the results since there exists tremendous growth in banks' assets in the past decades. To ensure the results concerning large banks are not determined by those mergers and 
acquisitions activities, an analysis for the large bank sample excluding banks whose have mergers and acquisitions activities during the sample period (Non-M\&A Banks) is conducted.

Panel E of Table 2-8 reports the regression results on the performance and market value of Non-M\&A large banks on their previous year's technology spending measures with each column as a separate regression for the dependent variable indicated in the column header. Consistent estimated coefficients for technology investment measures in Columns (1) to (4) suggest that technology make significant contributions in bank performance for those banks without large variations on their sizes. When the dependent variables are bank market valuation, the estimated parameters are positive but insignificant.

\subsubsection{Long Lags of Technology Spending Measures}

Panel F and Panel G of Table 2-8 report the regression results of Return on Assets and Return on Equity of banks on their previous one- to five-year technology spending measures using Equation (2-1) for large banks. Each column is a separate regression for the dependent variable indicated in the column header. The results indicate that bank performance of the large banks is associated with up to four-year lags of technology spending measures. These estimated coefficients are all positive and statistically significant at the $1 \%$ level. For instance, the coefficient for Tech Expenses/Total Assets is 1.22 when a two-year lag of the technology spending ratio is used. The $t$-statistics are highly 
statistically significant. These results suggest that long lags of technology spending measures have significant effects on bank performance.

2.5.5. Further Discussion on Endogeneity between Firm Performance and Technology Spending

Overall, the use of technology by banks is likely to be driven by a number of factors such as the rapid technological progress, socialization of the banking industry (Terry, Schwartz, and Schwartz, 2015), competition from non-banking institutions (Buchak, Matvos, Piskorski, and Seru, 2017), risk of information breaching' and financial regulations and compliance. Thus, one can argue that bank technology investments are largely exogenously driven. Just like other firms in the manufacturing and service industries, banks must meet challenges from the rapid advancement of technology, such as mobile banking, cloud computing, and data security, to stay competitive in the market regardless as to whether they are willing or able to adapt. To a large extent, the competitive market environment "forces" banks to increasingly invest in technology to catch up with the technology advancements.

Despite the argument and the attempts to address the potential endogeneity between bank performance and technology investments, the endogeneity concern is not completely eliminated. Further research should be conducted to provide additional evidence on this issue. Due to data limitations, it is challenging for us to employ other econometric techniques in empirical corporate finance to address the endogeneity issue (e.g., 
instrumental variables, difference-in-differences estimation, and regression discontinuity design). Thus, this paper provides some preliminary evidence regarding a possible causal effect of technology investments on bank performance.

\subsection{Conclusion}

The rapid adoption of technology by U.S. firms over the past two decades has drawn great attention from academics, policymakers, and practitioners. Despite the importance of technology investment, research regarding its impact on public firms has been limited. To fill this gap, this paper examines the effect of technology investment on firm performance and market value, using a unique dataset from the U.S. listed commercial banks from 2000-2017.

To meet the strong demand for agile and secure technology infrastructure and to stay competitive in the market, U.S. banks have significantly increased their technology spending during the past decades. This paper is one of the first studies to examine the extent to which expanded technology spending is related to financial performance and firm value, using technology spending data from the U.S. listed commercial banks.

First, a strong growth pattern in technology investment in the banking industry is documented. The technology spending of banks almost monotonically increased, even during the financial crisis. Banks experiencing negative performance shocks do not subsequently cut their technology spending. Thus, there is little evidence that bank 
technology spending is affected by negative performance shocks during the financial crisis and the slow revenue growth in recent years. These findings suggest that adoption of new technology has become a necessity instead of a strategic choice for banks, as they must provide quality services to customers and improve their productivity to remain competitive in the market.

Moreover, the research indicates that there is a strong positive correlation between bank performance and their lagged technology spending measures. This positive correlation is primarily driven by large banks. While greater technology spending increases Asset Turnover for both small and large banks, it only improves the Profit Margin of large banks implying that technology investments affect bank performance by improving operational efficiency, rather than by increasing sales. It appears that large banks are more likely to enjoy the economies of scale from lumpy technology investments. Consistent with Prasad and Harker (1997), there is little evidence that the financial performance of small banks is related to expanded technology spending, perhaps because small banks primarily adopt technology in order to survive and it is very costly for them to invest in technology, such as cybersecurity.

The findings in the paper can partly explain the "IT performance paradox" in the literature, as a size effect concerning the effects of technology spending on banks is identified. The results also indicate that technology investment helps increase bank value for large banks. The paper fills a gap in the literature as to whether the use of technology enhances or destroys firm value. 
This paper provides insights for managers with different firm sizes to make efficient capital allocation decisions. Also, the findings have implications for policymakers regarding changing regulations related to the use of technology. As large firms are more likely to gain benefits from lumpy technology investments, they are likely to have a significant competitive advantage in the market given the rapid growth in technological advances. This may lead to further consolidation in the banking industry and influence small business lending. Meanwhile, regulators should consider whether the current legal system, capital requirements, and cost models would facilitate firms in keeping up with the technological advances. Given the evidence that the effects of technology investment differ between small banks and large banks, it becomes a critical issue whether those regulations should be different for small firms and large firms.

As one of the first empirical studies examining the effects of technology investments on firm performance and firm value, there are some limitations in the paper. Additional research on the causal relationship between technology spending and firm performance is warranted. Also, it will be fruitful to examine relationships between technology investments and financing decisions, corporate governance, and firm risk on U.S. financial institutions. Finally, as the literature primarily consists of empirical analysis, there is a strong need for theoretical work to explain the effects of technology investments on corporate decisions. 


\section{References}

Acemoglu, D., Aghion, P., Zilibotti, F., 2006. Distance to frontier, selection, and economic growth. Journal of the European Economic Association 4(1), 37-74.

Adrian, T., Shin, H. S., 2009. Money, liquidity, and monetary policy. American Economic Review 99(2), 600-605.

Anderson, M. C., Banker, R. D., \& Ravindran, S., 2006. Value implications of investments in information technology. Management Science 52(9), 1359-1376.

Armstrong, C. S., Vashishtha, R., 2012. Executive stock options, differential risk-taking incentives, and firm value. Journal of Financial Economics 104(1), 70-88.

Arnaboldi, F., Claeys, P., 2010. Innovation and performance of European banks adopting Internet. Unpublished working paper. Cass Business School Working Paper Series.

Beccalli, E., 2007. Does it investment improve bank performance? Evidence from Europe. Journal of Banking and Finance 31(7), 2205-2230.

Berger, A. N., 2003. The economic effects of technological progress: Evidence from the banking industry. Journal of Money, Credit, and Banking 35(2), 141-176.

Berger, A. N., Bouwman, C. H., 2013. How does capital affect bank performance during financial crises? Journal of Financial Economics 109(1), 146-176.

Berger, A. N., DeYoung, R., 2006. Technological progress and the geographic expansion of the banking industry. Journal of Money, Credit and Banking, 38(6), 1483-1513.

Berger, A. N., Udell, G. F., 2002. Small business credit availability and relationship lending: The importance of bank organizational structure. The Economic Journal 112(477), F32-F53.

Berndt, E. R., Morrison, C. J., 1995. High-tech capital formation and economic performance in U.S. manufacturing industries an exploratory analysis. Journal of Econometrics 65(1), 9-43.

Brigham, E. F., Daves, P. R., 2014. Intermediate Financial Management. Cengage Learning

Brynjolfsson, E., 1993. The productivity paradox of information technology. Communications of the ACM 36(12), 66-77.

Brynjolfsson, E., Yang, S., 1996. Information technology and productivity: A review of the literature. Advances in Computers, 43, 179-214. 
Buchak, G., Matvos, G., Piskorski, T., Seru, A., 2017. FinTech, regulatory arbitrage, and the rise of shadow banks. National Bureau of Economic Research Working Paper (No. w23288).

Cheng, I. H., Hong, H., Scheinkman, J. A., 2015. Yesterday's heroes: Compensation and risk at financial firms. Journal of Finance 70(2), 839-879.

Dahl, D., Meyer, A., \& Wiggins, N. (2017). How fast will banks adopt new technology this time? The Regional Economist. Fourth Quarter 2017.

DeYoung, R., Lang, W. W., Nolle, D. L., 2007. How the Internet affects output and performance at community banks. Journal of Banking and Finance 31(4), 1033-1060.

Draca, M., Sadun, R., Van Reenen, J., 2007. Productivity and ICT: A review of the evidence. In: Mansell, R. (Eds.), The Oxford Handbook of Information and Communication Technologies. Oxford, UK, Oxford University Press, 100-147.

Elsas, R., Hackethal, A., Holzhäuser, M., 2010. The anatomy of bank diversification. Journal of Banking and Finance 34(6), 1274-1287.

Ferrari, S., Verboven, F., Degryse, H., 2010. Investment and usage of new technologies: Evidence from a shared ATM network. American Economic Review 100(3), 10461079.

Fiordelisi, F., Molyneux, P., 2010. The determinants of firm value in European banking. Journal of Banking and Finance 34(6), 1189-1200.

Frame, W. S., White, L. J., 2014. Technological change, financial innovation, and diffusion in banking. In: Berger, A. N., Molyneux, P., and Wilson, J. O. S. (Ed.) The Oxford Handbook of Banking, 2nd Edition, Oxford University Press, Oxford, UK.

Gali, J.. 1999. Technology, employment, and the business cycle: Do technology shocks explain aggregate fluctuations? American Economic Review 89(1), 249-271.

Greenwood, J., Hercowitz, Z., Huffman, G. W., 1988. Investment, capacity utilization, and the real business cycle. American Economic Review 78(3), 402-417.

Greenwood, J., Hercowitz, Z., Krusell, P., 1997. Long-run implications of investmentspecific technological change American Economic Review 87(3), 342-362.

Grossman, G. M., Helpman, E., 1994. Endogenous innovation in theory of growth. Journal of Economic Perspectives 8(1), 23-44. 
Hall, B. H. H., Khan, B., 2003. New Economy Handbook, Chapter Adoption of New Technology. Elsevier Science, Amsterdam.

Hansen, G. D., Prescott, E. C., 2002. Malthus to Solow. American Economic Review 92(4), 1205-1217.

Harris, S. E., Katz, J. L., 1991. Firm size and the information technology investment intensity of life insurers. MIS Quarterly 15(3), 333-352.

Haynes, M., Thompson, S., 2000. The productivity impact of IT deployment: An empirical evaluation of ATM introduction. Oxford Bulletin of Economics and Statistics 62(5), 607-619.

Healy, P. M., Palepu, K. G., 2012. Business Analysis Valuation: Using Financial Statements. Cengage Learning.

Hernández-Murillo, R., Llobet, G., Fuentes, R., 2010. Strategic online banking adoption. Journal of Banking and Finance 34(7), 1650-1663.

Hitt, L. M., Brynjolfsson, E., 1996. Productivity, business performance, and consumer surplus: Three different measures of information technology value. MIS Quarterly 20(2), 121-142.

Hunter, W. C., Timme, S. G., 1986. Technical change, organizational form, and the structure of bank production. Journal of Money, Credit and Banking 18(2), 152-166.

Ittner, C. D., Larcker, D. F., 2001. Assessing empirical research in managerial accounting: A value-based management perspective. Journal of Accounting and Economics 32(1), $349-410$.

Jansen, I. P., Ramnath, S., Yohn, T. L., 2012. A diagnostic for earnings management using changes in asset turnover and profit margin. Contemporary Accounting Research 29(1), 221-251.

Katz, M. L., Shapiro, C., 1986. Technology adoption in the presence of network externalities. Journal of Political Economy 94(4), 822-841.

Kydland, F. E., Prescott, E. C., 1982. Time to build and aggregate fluctuations. Econometrica 50(6), 1345-1370.

Lamont, O. A., Polk, C., 2002. Does diversification destroy value? Evidence from the industry shocks. Journal of Financial Economics 63(1), 51-77.

Madsen, J. B., 2014. Human capital and the world technology frontier. Review of Economics and Statistics 96(4), 676-692. 
Marcus, A. J., 1984. Deregulation and bank financial policy. Journal of Banking and Finance 8(4), 557-565.

Martín-Oliver, A., Ruano, S., Salas-Fumás, V., 2013. Why high productivity growth of banks preceded the financial crisis. Journal of Financial Intermediation 22(4), 688-712.

Martín-Oliver, A., Salas-Fumás, V., 2008. The output and profit contribution of information technology and advertising investments in banks. Journal of Financial Intermediation 17(2), 229-255.

Mehran, H., Thakor, A., 2011. Bank capital and value in the cross-section. Review of Financial Studies 24(4), 1019-1067.

Melnick, E. L., Nayyar, P. R., Pinedo, M. L., Seshadri, S., 2000. Creating value in financial services. In: Creating Value in Financial Services. San Francisco, CA, Springer. 1-22.

Mills, D., Wang, K., Malone, B., Ravi, A., Marquardt, J., Chen, C., Badev, A., Brezinski, T., Fahy, L., Liao, K., Kargenian, V., Ellithorpe, M., Ng, W., Baird, M., 2016. Distributed ledger technology in payments, clearing, and settlement, Finance and Economics Discussion Series 2016-095. Washington: Board of Governors of the Federal Reserve System.

Mithas, S., Tafti, A., Bardhan, I., Goh, J. M., 2012. Information technology and firm performance: Mechanisms and empirical evidence. MIS Quarterly 36(1), 205-224.

Muscarella, C. J., Vetsuypens, M. R., 1990. Efficiency and organizational structure: A study of reverse LBOs. Journal of Finance 45(5), 1389-1413.

Myers, S. C., Majluf, N. S., 1984. Corporate financing and investment decisions when firms have information that investors do not have. Journal of Financial Economics 13(2), 187-221.

Pang, H., 2018, The impact of information technology on U.S. commercial banks: Who is the winner/loser? Unpublished working paper. Arizona State University.

Petersen, M. A., Rajan, R. G., 2002. Does distance still matter? The information revolution in small business lending. Journal of Finance 57(6), 2533-2570.

Philippon, T., 2015. Has the U.S. finance industry become less efficient? On theory and measurement of financial intermediation. American Economic Review 105(4), 14081438 . 
Prasad, B., Harker, P. T., 1997. Examining the contribution of information technology toward productivity and performance in U.S. retail banking. Unpublished Working paper. The Wharton Financial Institutions Center Working Papers 97(9).

Saloner, G., Shepard, A., 1995. Adoption of technologies with network effects: An empirical examination of the adoption of teller machines. RAND Journal of Economics 26(3), 479-501.

Sealey, C. W., Lindley, J. T., 1977. Inputs, outputs, and a theory of production and cost at depository financial institutions. Journal of Finance 32(4), 1251-1266.

Stulz, R., 1990. Managerial discretion and optimal financing policies. Journal of Financial Economics 26(1), 3-27.

Sullivan, R. J., Wang, Z, 2013. Internet Banking: An Exploration in Technology Diffusion and Impact. FRB Richmond Working Paper No. 13-10.

Terry, H. M., Schwartz, D., Schwartz, T., 2015. The Future of Finance: The Socialization of Finance. Goldman Sachs Investment Research, 3.

Titman, S., Keown, A. J., Martin, J. D., 2013. Financial Management: Principles and Applications (Vol. 12). Pearson.

Triplett, J. E., Bosworth, B. P., 2006. 'Baumol's disease' has been cured: IT and multifactor productivity in U.S. services industries, The New Economy and Beyond: Past, Present, and Future, 34-71. 
Table 2.1. Summary Statistics

This table reports the summary statistics of the key variables used in this paper. All of the variables are defined in Table A1 in the appendix. Since lagged variables are included in regressions, firms with fewer than two consecutive years of technology expenses and total assets information are excluded. The variables have been winsorized at the $1 \%$ and $99 \%$ tails of the distributions to avoid the influence of extreme observations.

\begin{tabular}{lllllll}
\hline Variables & Mean & $\begin{array}{l}\text { Media } \\
\mathrm{n}\end{array}$ & $\begin{array}{l}\text { Std. } \\
\text { Dev. }\end{array}$ & Min & Max & Obs. \\
\hline Total Assets (\$B) & & & & & & \\
Market Capitalization (\$B) & 1.143 & 0.105 & 4.963 & 0.004 & 42.479 & 8,343 \\
Technology Expense (\$M) & 14.305 & 1.504 & 70.077 & 0.117 & 598.000 & 8,706 \\
Leverage & 11.159 & 10.556 & 4.053 & 4.282 & 32.467 & 8,706 \\
Loans/Assets & 0.667 & 0.682 & 0.124 & 0.257 & 0.892 & 8,706 \\
Deposits/Assets & 0.768 & 0.788 & 0.092 & 0.468 & 0.908 & 8,706 \\
Deposits/Liability & 0.856 & 0.879 & 0.102 & 0.510 & 0.995 & 8,706 \\
Return on Assets (\%) & 0.816 & 0.942 & 0.827 & -3.425 & 2.456 & 7,974 \\
Return on Equity (\%) & 8.282 & 9.773 & 11.894 & -67.231 & 30.417 & 7,974 \\
Profit Margin (\%) & 18.874 & 23.424 & 21.291 & -103.491 & 47.813 & 7,974 \\
Asset Turnover (\%) & 4.178 & 4.080 & 1.033 & 1.955 & 8.131 & 8,703 \\
Market-to-Book Equity Ratio & 1.330 & 1.212 & 0.635 & 0.231 & 3.603 & 8,308 \\
Firm $Q$ & 1.030 & 1.021 & 0.056 & 0.932 & 1.224 & 8,343 \\
Tech Expense/Total Assets (\%) & 0.180 & 0.166 & 0.097 & 0.025 & 0.486 & 8,706 \\
Tech Expense/Loans \& Deposits & 0.127 & 0.116 & 0.069 & 0.020 & 0.369 & 8,706 \\
(\%) & & & & & & \\
\hline
\end{tabular}


Table 2.2. Return on Assets and Technology Investment

This table reports the results of the regressions of bank Return on Assets on their technology investment measures (Tech Expense/Total Assets, and Tech Expense/Loans \& Deposits) in the previous year in the sample from 2000-2017. The standard errors are clustered at the firm level. $t$-statistics based on robust standard errors are in brackets. Significance at the $1 \%, 5 \%$, or $10 \%$ levels are presented as*, **, or ***, respectively. All of the variables are defined in Table A1 in the appendix. Since lagged variables are included in regressions, firms with fewer than two consecutive years of technology expenses and total assets information are excluded. The variables have been winsorized at the $1 \%$ and $99 \%$ tails of the distributions to avoid the influence of extreme observations.

\begin{tabular}{|c|c|c|c|c|c|c|}
\hline Variables & $\begin{array}{c}(1) \\
\text { All } \\
\text { Banks }\end{array}$ & $\begin{array}{c}\text { (2) } \\
\text { All } \\
\text { Banks } \\
\end{array}$ & $\begin{array}{c}(3) \\
\text { Small } \\
\text { Banks } \\
\end{array}$ & $\begin{array}{c}4) \\
\text { Small } \\
\text { Banks }\end{array}$ & $\begin{array}{c}(5) \\
\text { Large } \\
\text { Banks } \\
\end{array}$ & $\begin{array}{c}6) \\
\text { Large } \\
\text { Banks }\end{array}$ \\
\hline \multirow[t]{2}{*}{ Tech Expense/Total Assets, $t-1$} & $0.604^{*}$ & & 0.128 & & $\begin{array}{c}0.927 * * \\
*\end{array}$ & \\
\hline & {$[1.90]$} & & {$[0.24]$} & & {$[3.10]$} & \\
\hline \multirow[t]{2}{*}{ Tech Expense/Loans \& Deposits, $t-1$} & & $0.853^{*}$ & & 0.194 & & $\begin{array}{c}1.317 * * \\
*\end{array}$ \\
\hline & & {$[1.84]$} & & {$[0.24]$} & & {$[3.21]$} \\
\hline \multirow[t]{2}{*}{ Log Market Capitalization, $t-1$} & $\begin{array}{c}0.321^{* *} \\
*\end{array}$ & $\begin{array}{c}0.321^{* *} \\
*\end{array}$ & $\begin{array}{c}0.326^{* *} \\
*\end{array}$ & $\begin{array}{c}0.326^{* *} \\
*\end{array}$ & $\begin{array}{c}0.329 * * \\
*\end{array}$ & $\begin{array}{c}0.328 * * \\
*\end{array}$ \\
\hline & {$[8.58]$} & {$[8.55]$} & {$[4.25]$} & {$[4.23]$} & [7.64] & [7.60] \\
\hline \multirow[t]{2}{*}{ Leverage, $t-1$} & $\begin{array}{c}- \\
0.015^{* *}\end{array}$ & $0.015^{-}$ & -0.013 & -0.013 & $-0.018^{*}$ & $-0.018 *$ \\
\hline & {$[-2.06]$} & {$[-2.05]$} & {$[-1.10]$} & {$[-1.10]$} & [-1.79] & {$[-1.78]$} \\
\hline Loans/Assets, $t-1$ & $\begin{array}{l}-0.048 \\
{[-0.24]}\end{array}$ & $\begin{array}{c}0.027 \\
{[0.13]}\end{array}$ & $\begin{array}{c}0.380 \\
{[1.15]}\end{array}$ & $\begin{array}{c}0.397 \\
{[1.23]}\end{array}$ & $\begin{array}{c}-0.404 * \\
{[-1.78]}\end{array}$ & $\begin{array}{c}-0.291 \\
{[-1.28]}\end{array}$ \\
\hline Deposits/Assets, $t-1$ & $\begin{array}{l}1.608^{*} \\
{[1.81]}\end{array}$ & $\begin{array}{l}1.689^{*} \\
{[1.90]}\end{array}$ & $\begin{array}{c}1.513 \\
{[1.26]}\end{array}$ & $\begin{array}{l}1.531 \\
{[1.27]}\end{array}$ & $\begin{array}{l}1.280 \\
{[0.98]}\end{array}$ & $\begin{array}{l}1.403 \\
{[1.07]}\end{array}$ \\
\hline Deposits/Liability, $t-1$ & $\begin{array}{l}-1.122 \\
{[-1.43]}\end{array}$ & $\begin{array}{l}-1.131 \\
{[-1.44]}\end{array}$ & $\begin{array}{l}-0.796 \\
{[-0.73]}\end{array}$ & $\begin{array}{l}-0.799 \\
{[-0.73]}\end{array}$ & $\begin{array}{l}-1.094 \\
{[-0.95]}\end{array}$ & $\begin{array}{l}-1.110 \\
{[-0.97]}\end{array}$ \\
\hline Constant & $\begin{array}{l}-0.330 \\
{[-1.06]}\end{array}$ & $\begin{array}{l}-0.431 \\
{[-1.35]}\end{array}$ & $\begin{array}{l}-0.602 \\
{[-1.23]}\end{array}$ & $\begin{array}{l}-0.628 \\
{[-1.26]}\end{array}$ & $\begin{array}{l}-0.112 \\
{[-0.28]}\end{array}$ & $\begin{array}{l}-0.265 \\
{[-0.65]}\end{array}$ \\
\hline Observations & 6,652 & 6,652 & 3,060 & 3,060 & 3,592 & 3,592 \\
\hline Number of Banks & 912 & 912 & 463 & 463 & 449 & 449 \\
\hline Adjusted $R$-squared & 0.293 & 0.293 & 0.249 & 0.249 & 0.334 & 0.334 \\
\hline Bank Fixed Effects & YES & YES & YES & YES & YES & YES \\
\hline Year Fixed Effects & YES & YES & YES & YES & YES & YES \\
\hline
\end{tabular}


Table 2.3. Return on Equity and Technology Investment

This table reports the results of the regressions of bank Return on Equity on their technology investment measures (Tech Expense/Total Assets, and Tech Expense/Loans \& Deposits) in the previous year in the sample from 2000-2017. The standard errors are clustered at the firm level. $t$-statistics based on robust standard errors are in brackets. Significance at the $1 \%, 5 \%$, or $10 \%$ levels are presented as*, **, or ***, respectively. All of the variables are defined in Table A1 in the appendix. Since lagged variables are included in regressions, firms with fewer than two consecutive years of technology expenses and total assets information are excluded. The variables have been winsorized at the $1 \%$ and $99 \%$ tails of the distributions to avoid the influence of extreme observations.

\begin{tabular}{|c|c|c|c|c|c|c|}
\hline Variables & $\begin{array}{c}(1) \\
\text { All } \\
\text { Banks } \\
\end{array}$ & $\begin{array}{c}(2) \\
\text { All } \\
\text { Banks } \\
\end{array}$ & $\begin{array}{c}(3) \\
\text { Small } \\
\text { Banks } \\
\end{array}$ & $\begin{array}{c}(4) \\
\text { Small } \\
\text { Banks } \\
\end{array}$ & $\begin{array}{c}(5) \\
\text { Large } \\
\text { Banks } \\
\end{array}$ & $\begin{array}{c}(6) \\
\text { Large } \\
\text { Banks } \\
\end{array}$ \\
\hline Tech Expense/Total Assets, $t-1$ & $\begin{array}{c}10.595 * \\
* \\
{[2.03]}\end{array}$ & & $\begin{array}{l}4.078 \\
{[0.47]}\end{array}$ & & $\begin{array}{c}15.752 * \\
* * \\
{[3.25]}\end{array}$ & \\
\hline Tech Expense/Loans \& Deposits, $t-1$ & & $\begin{array}{c}15.135 * \\
* \\
{[1.98]}\end{array}$ & $=$ & $\begin{array}{l}6.829 \\
{[0.52]}\end{array}$ & & $\begin{array}{c}22.112 * \\
* * \\
{[3.32]}\end{array}$ \\
\hline Log Market Capitalization, $t-1$ & $\begin{array}{c}4.046 * * \\
* \\
{[5.34]}\end{array}$ & $\begin{array}{c}4.040 * * \\
* \\
{[5.32]}\end{array}$ & $\begin{array}{c}4.690 * * \\
* \\
{[3.35]}\end{array}$ & $\begin{array}{c}4.715 * * \\
* \\
{[3.35]}\end{array}$ & $\begin{array}{c}4.054 * * \\
* \\
{[4.46]}\end{array}$ & $\begin{array}{c}4.034 * * \\
* \\
{[4.44]}\end{array}$ \\
\hline Leverage, $t-1$ & $\begin{array}{l}-0.156 \\
{[-0.94]}\end{array}$ & $\begin{array}{l}-0.156 \\
{[-0.94]}\end{array}$ & $\begin{array}{l}-0.200 \\
{[-0.70]}\end{array}$ & $\begin{array}{l}-0.200 \\
{[-0.70]}\end{array}$ & $\begin{array}{l}-0.125 \\
{[-0.66]}\end{array}$ & $\begin{array}{l}-0.123 \\
{[-0.65]}\end{array}$ \\
\hline Loans/Assets, $t-1$ & $\begin{array}{l}-2.954 \\
{[-0.94]}\end{array}$ & $\begin{array}{l}-1.627 \\
{[-0.52]}\end{array}$ & $\begin{array}{c}1.997 \\
{[0.38]}\end{array}$ & $\begin{array}{l}2.604 \\
{[0.50]}\end{array}$ & $\begin{array}{c}-6.546^{*} \\
{[-1.81]}\end{array}$ & $\begin{array}{l}-4.646 \\
{[-1.29]}\end{array}$ \\
\hline Deposits/Assets, $t-1$ & $\begin{array}{c}56.219 * \\
* * \\
{[3.64]}\end{array}$ & $\begin{array}{c}57.649 * \\
* * \\
{[3.72]}\end{array}$ & $\begin{array}{c}46.662 * \\
* \\
{[2.17]}\end{array}$ & $\begin{array}{c}47.319 * \\
* \\
{[2.19]}\end{array}$ & $\begin{array}{c}61.743 * \\
* * \\
{[2.66]}\end{array}$ & $\begin{array}{c}63.787 * \\
* * \\
{[2.75]}\end{array}$ \\
\hline Deposits/Liab & $\begin{array}{c}- \\
47.610 * \\
* *\end{array}$ & $\begin{array}{c}- \\
47.783 * \\
* *\end{array}$ & -31.093 & -31.226 & $\begin{array}{c}- \\
60.008 * \\
* *\end{array}$ & $\begin{array}{c}- \\
60.247 * \\
* *\end{array}$ \\
\hline Constant & $\begin{array}{l}{[-3.41]} \\
-4.014 \\
{[-0.73]}\end{array}$ & $\begin{array}{l}{[-3.42]} \\
-5.829 \\
{[-1.01]}\end{array}$ & $\begin{array}{c}{[-1.63]} \\
- \\
12.709^{*} \\
{[-1.81]}\end{array}$ & $\begin{array}{c}{[-1.64]} \\
- \\
13.696^{*} \\
{[-1.81]}\end{array}$ & $\begin{array}{c}{[-2.86]} \\
1.160 \\
{[0.15]}\end{array}$ & $\begin{array}{l}{[-2.87]} \\
-1.362 \\
{[-0.17]}\end{array}$ \\
\hline $\begin{array}{l}\text { Number of Observations } \\
\text { Number of Banks } \\
\text { Adjusted } R \text {-squared } \\
\text { Bank Fixed Effects } \\
\text { Year Fixed Effects }\end{array}$ & $\begin{array}{l}6,652 \\
912 \\
0.208 \\
\text { YES } \\
\text { YES }\end{array}$ & $\begin{array}{c}6,652 \\
912 \\
0.208 \\
\text { YES } \\
\text { YES }\end{array}$ & $\begin{array}{l}3,060 \\
463 \\
0.168 \\
\text { YES } \\
\text { YES } \\
\end{array}$ & $\begin{array}{l}3,060 \\
463 \\
0.168 \\
\text { YES } \\
\text { YES }\end{array}$ & $\begin{array}{c}3,592 \\
449 \\
0.250 \\
\text { YES } \\
\text { YES }\end{array}$ & $\begin{array}{c}3,592 \\
449 \\
0.250 \\
\text { YES } \\
\text { YES } \\
\end{array}$ \\
\hline
\end{tabular}


Table 2.4. Profit Margin and Technology Investment

This table reports the results of the regressions of bank Profit Margin on their technology investment measures (Tech Expense/Total Assets, and Tech Expense/Loans \& Deposits) in the previous year in the sample from 2000-2017. The standard errors are clustered at the firm level. $t$-statistics based on robust standard errors are in brackets. Significance at the $1 \%, 5 \%$, or $10 \%$ levels are presented as*, **, or ***, respectively. All of the variables are defined in Table A1 in the appendix. Since lagged variables are included in regressions, firms with fewer than two consecutive years of technology expenses and total assets information are excluded. The variables have been winsorized at the $1 \%$ and $99 \%$ tails of the distributions to avoid the influence of extreme observations.

\begin{tabular}{|c|c|c|c|c|c|c|}
\hline Variables & $\begin{array}{c}(1) \\
\text { All } \\
\text { Banks } \\
\end{array}$ & $\begin{array}{c}(2) \\
\text { All } \\
\text { Banks } \\
\end{array}$ & $\begin{array}{c}(3) \\
\text { Small } \\
\text { Banks } \\
\end{array}$ & $\begin{array}{c}(4) \\
\text { Small } \\
\text { Banks } \\
\end{array}$ & $\begin{array}{c}(5) \\
\text { Large } \\
\text { Banks } \\
\end{array}$ & $\begin{array}{c}6) \\
\text { Large } \\
\text { Banks } \\
\end{array}$ \\
\hline Tech Expense/Total Assets, $t-1$ & $\begin{array}{l}6.903 \\
{[0.86]}\end{array}$ & & $\begin{array}{l}-2.981 \\
{[-0.21]}\end{array}$ & & $\begin{array}{c}14.571 * \\
* \\
{[2.02]}\end{array}$ & \\
\hline Tech Expense/Loans \& Deposits, $t-1$ & & $\begin{array}{l}8.449 \\
{[0.73]}\end{array}$ & & $\begin{array}{l}-5.180 \\
{[-0.25]}\end{array}$ & & $\begin{array}{c}19.399 * \\
{[1.95]}\end{array}$ \\
\hline Log Market Capitalization, $t-1$ & $\begin{array}{c}7.706 * * \\
* \\
{[7.51]}\end{array}$ & $\begin{array}{c}7.679 * * \\
* \\
{[7.47]}\end{array}$ & $\begin{array}{c}7.612 * * \\
* \\
{[3.48]}\end{array}$ & $\begin{array}{c}7.589 * * \\
* \\
{[3.46]}\end{array}$ & $\begin{array}{c}7.772 * * \\
* \\
{[6.90]}\end{array}$ & $\begin{array}{c}7.740 * * \\
* \\
{[6.85]}\end{array}$ \\
\hline Leverage, $t-1$ & $\begin{array}{c}- \\
0.426 * * \\
{[-2.03]}\end{array}$ & $\begin{array}{c}- \\
0.427 * * \\
{[-2.03]}\end{array}$ & $\begin{array}{l}-0.452 \\
{[-1.37]}\end{array}$ & $\begin{array}{l}-0.452 \\
{[-1.37]}\end{array}$ & $\begin{array}{l}-0.427 \\
{[-1.55]}\end{array}$ & $\begin{array}{l}-0.426 \\
{[-1.55]}\end{array}$ \\
\hline Loans/Assets, $t-1$ & -1.265 & -0.529 & 11.330 & 10.869 & $1 \stackrel{-}{-}^{1}{ }^{*}$ & -10.151 \\
\hline Deposits/Assets, $t-1$ & $\begin{array}{c}{[-0.23]} \\
45.438 * \\
{[1.94]}\end{array}$ & $\begin{array}{c}{[-0.10]} \\
46.195^{*} \\
{[1.96]}\end{array}$ & $\begin{array}{c}{[1.22]} \\
45.702 \\
{[1.50]}\end{array}$ & $\begin{array}{c}{[1.19]} \\
45.201 \\
{[1.48]}\end{array}$ & $\begin{array}{c}{[-1.82]} \\
43.424 \\
{[1.21]}\end{array}$ & $\begin{array}{c}{[-1.55]} \\
45.148 \\
{[1.26]}\end{array}$ \\
\hline Deposits/Liability, $t-1$ & $\begin{array}{c}- \\
40.870 * \\
*\end{array}$ & $\begin{array}{c}- \\
40.817 * \\
*\end{array}$ & -30.407 & -30.293 & -47.759 & -47.822 \\
\hline Constant & $\begin{array}{l}{[-1.97]} \\
-1.310 \\
{[-0.16]}\end{array}$ & $\begin{array}{l}{[-1.97]} \\
-2.156 \\
{[-0.25]}\end{array}$ & $\begin{array}{c}{[-1.10]} \\
-10.312 \\
{[-0.81]}\end{array}$ & $\begin{array}{l}{[-1.09]} \\
-9.543 \\
{[-0.71]}\end{array}$ & $\begin{array}{c}{[-1.53]} \\
5.670 \\
{[0.54]}\end{array}$ & $\begin{array}{c}{[-1.53]} \\
3.592 \\
{[0.33]}\end{array}$ \\
\hline $\begin{array}{l}\text { Number of Observations } \\
\text { Number of Banks } \\
\text { Adjusted } R \text {-squared } \\
\text { Bank Fixed Effects } \\
\text { Year Fixed Effects }\end{array}$ & $\begin{array}{l}6,652 \\
912 \\
0.277 \\
\text { YES } \\
\text { YES }\end{array}$ & $\begin{array}{c}6,652 \\
912 \\
0.277 \\
\text { YES } \\
\text { YES }\end{array}$ & $\begin{array}{l}3,060 \\
463 \\
0.242 \\
\text { YES } \\
\text { YES }\end{array}$ & $\begin{array}{l}3,060 \\
463 \\
0.242 \\
\text { YES } \\
\text { YES }\end{array}$ & $\begin{array}{c}3,592 \\
449 \\
0.311 \\
\text { YES } \\
\text { YES }\end{array}$ & $\begin{array}{c}3,592 \\
449 \\
0.311 \\
\text { YES } \\
\text { YES }\end{array}$ \\
\hline
\end{tabular}


Table 2.5. Asset Turnover and Technology Investment

This table reports the results of the regressions of bank Asset Turnover on their technology investment measures (Tech Expense/Total Assets, and Tech Expense/Loans \& Deposits) in the previous year in the sample from 2000-2017. The standard errors are clustered at the firm level. $t$-statistics based on robust standard errors are in brackets. Significance at the $1 \%, 5 \%$, or $10 \%$ levels are presented as $* * *$, or $* * *$, respectively. All of the variables are defined in Table A1 in the appendix. Since lagged variables are included in regressions, firms with fewer than two consecutive years of technology expenses and total assets information are excluded. The variables have been winsorized at the $1 \%$ and $99 \%$ tails of the distributions to avoid the influence of extreme observations.

\begin{tabular}{|c|c|c|c|c|c|c|}
\hline Variables & $\begin{array}{c}\text { (1) } \\
\text { All } \\
\text { Banks }\end{array}$ & $\begin{array}{c}(2) \\
\text { All } \\
\text { Banks } \\
\end{array}$ & $\begin{array}{c}(3) \\
\text { Small } \\
\text { Banks }\end{array}$ & $\begin{array}{c}(4) \\
\text { Small } \\
\text { Banks }\end{array}$ & $\begin{array}{c}5) \\
\text { Large } \\
\text { Banks } \\
\end{array}$ & $\begin{array}{c}6) \\
\text { Large } \\
\text { Banks } \\
\end{array}$ \\
\hline Tech Expense/Total Assets, $t-1$ & $\begin{array}{c}1.880 * * * \\
{[6.14]}\end{array}$ & & $\begin{array}{c}1.639^{* * * *} \\
{[4.31]}\end{array}$ & & $\begin{array}{c}1.970 * * * \\
{[4.17]}\end{array}$ & \\
\hline Tech Expense/Loans \& Deposits, $t-1$ & & $\begin{array}{c}2.775 * * * \\
{[6.29]}\end{array}$ & & $\begin{array}{c}2.448 * * * \\
{[4.15]}\end{array}$ & & $\begin{array}{c}2.892^{* * * *} \\
{[4.48]}\end{array}$ \\
\hline Log Market Capitalization, $t-1$ & $\begin{array}{c}0.107 * * * \\
{[2.63]}\end{array}$ & $\begin{array}{c}0.108 * * * * \\
{[2.66]}\end{array}$ & $\begin{array}{c}0.132 * * \\
{[2.52]}\end{array}$ & $\begin{array}{c}0.136 * * * \\
{[2.63]}\end{array}$ & $\begin{array}{c}0.117 * * \\
{[2.06]}\end{array}$ & $=0.117 * *$ \\
\hline Leverage, $t-1$ & $\begin{array}{l}0.006 \\
{[0.82]}\end{array}$ & $\begin{array}{l}0.006 \\
{[0.82]}\end{array}$ & $\begin{array}{l}0.019^{*} \\
{[1.81]}\end{array}$ & $\begin{array}{l}0.019^{*} \\
{[1.80]}\end{array}$ & $\begin{array}{l}-0.002 \\
{[-0.22]}\end{array}$ & $\begin{array}{l}-0.002 \\
{[-0.21]}\end{array}$ \\
\hline Loans/Assets, $t-1$ & $\begin{array}{l}0.517^{*} \\
{[1.74]}\end{array}$ & $\begin{array}{c}0.759^{* *} \\
{[2.50]}\end{array}$ & $\begin{array}{c}0.638^{* *} \\
{[2.03]}\end{array}$ & $\begin{array}{c}0.857 * * * \\
{[2.82]}\end{array}$ & $\begin{array}{l}0.436 \\
{[0.91]}\end{array}$ & $\begin{array}{l}0.681 \\
{[1.39]}\end{array}$ \\
\hline Deposits/Assets, $t-1$ & $\begin{array}{l}-0.247 \\
{[-0.21]}\end{array}$ & $\begin{array}{l}0.028 \\
{[0.02]}\end{array}$ & $\begin{array}{l}-1.016 \\
{[-0.65]}\end{array}$ & $\begin{array}{l}-0.782 \\
{[-0.50]}\end{array}$ & $\begin{array}{l}-0.242 \\
{[-0.13]}\end{array}$ & $\begin{array}{l}0.054 \\
{[0.03]}\end{array}$ \\
\hline Deposits/Liability, $t-1$ & $\begin{array}{c}1.502 \\
{[1.48]}\end{array}$ & $\begin{array}{l}1.445 \\
{[1.43]}\end{array}$ & $\begin{array}{l}1.765 \\
{[1.22]}\end{array}$ & $\begin{array}{c}1.734 \\
{[1.21]}\end{array}$ & $\begin{array}{l}1.671 \\
{[1.13]}\end{array}$ & $\begin{array}{l}1.594 \\
{[1.08]}\end{array}$ \\
\hline Consta & $\begin{array}{c}2.246 * * * \\
{[4.67]}\end{array}$ & $\begin{array}{c}1.907 * * * \\
{[3.91]}\end{array}$ & $\begin{array}{c}2.174 * * * \\
{[3.93]}\end{array}$ & $\begin{array}{c}1.850 * * * \\
{[3.46]}\end{array}$ & $\begin{array}{c}2.321 * * * \\
{[3.05]}\end{array}$ & $\begin{array}{c}* 1.983 * * \\
{[2.54]}\end{array}$ \\
\hline $\begin{array}{l}\text { Number of Observations } \\
\text { Number of Banks } \\
\text { Adjusted } R \text {-squared } \\
\text { Bank Fixed Effects } \\
\text { Year Fixed Effects }\end{array}$ & $\begin{array}{l}7,291 \\
963 \\
0.136 \\
\text { YES } \\
\text { YES }\end{array}$ & $\begin{array}{l}7,291 \\
963 \\
0.139 \\
\text { YES } \\
\text { YES }\end{array}$ & $\begin{array}{c}3,271 \\
481 \\
0.123 \\
\text { YES } \\
\text { YES }\end{array}$ & $\begin{array}{l}481 \\
0.125 \\
\text { YES } \\
\text { YES }\end{array}$ & $\begin{array}{c}4,020 \\
482 \\
0.156 \\
\text { YES } \\
\text { YES }\end{array}$ & $\begin{array}{l}4,020 \\
482 \\
0.158 \\
\text { YES } \\
\text { YES }\end{array}$ \\
\hline
\end{tabular}


Table 2.6. Market-to-Book Equity Ratio and Technology Investment

This table reports the results of the regressions of bank Market-to-Book Equity Ratio on their technology investment measures (Tech Expense/Total Assets, and Tech Expense/Loans \& Deposits) in the previous year in the sample from 2000-2017. The standard errors are clustered at the firm level. $t$-statistics based on robust standard errors are in brackets. Significance at the $1 \%, 5 \%$, or $10 \%$ levels are presented as*, **, or ***, respectively. All of the variables are defined in Table A1 in the appendix. Since lagged variables are included in regressions, firms with fewer than two consecutive years of technology expenses and total assets information are excluded. The variables have been winsorized at the $1 \%$ and $99 \%$ tails of the distributions to avoid the influence of extreme observations.

\begin{tabular}{|c|c|c|c|c|c|c|}
\hline Variables & $\begin{array}{c}(1) \\
\text { All } \\
\text { Banks } \\
\end{array}$ & $\begin{array}{c}\text { (2) } \\
\text { All } \\
\text { Banks } \\
\end{array}$ & $\begin{array}{c}(3) \\
\text { Small } \\
\text { Banks } \\
\end{array}$ & $\begin{array}{c}(4) \\
\text { Small } \\
\text { Banks }\end{array}$ & $\begin{array}{c}(5) \\
\text { Large } \\
\text { Banks } \\
\end{array}$ & $\begin{array}{c}\text { (6) } \\
\text { Large } \\
\text { Banks } \\
\end{array}$ \\
\hline Tech Expense/Total Assets, $t-1$ & $\begin{array}{l}0.249 \\
{[1.64]}\end{array}$ & & $\begin{array}{l}-0.117 \\
{[-0.63]}\end{array}$ & & $\begin{array}{c}0.526 * * \\
{[2.39]}\end{array}$ & \\
\hline Tech Expense/Loans \& Deposits, $t-1$ & & $\begin{array}{l}0.299 \\
{[1.42]}\end{array}$ & & $\begin{array}{l}-0.162 \\
{[-0.61]}\end{array}$ & & $\begin{array}{c}0.652 * * \\
{[2.20]}\end{array}$ \\
\hline Log Market Capitalization, $t-1$ & $\begin{array}{c}0.249 * * \\
* \\
{[9.98]}\end{array}$ & $\begin{array}{c}0.248^{* *} \\
* \\
{[9.96]}\end{array}$ & $\begin{array}{c}0.212 * * \\
* \\
{[4.41]}\end{array}$ & $\begin{array}{c}0.212 * * \\
* \\
{[4.43]}\end{array}$ & $\begin{array}{c}0.286^{* *} * \\
* \\
{[9.43]}\end{array}$ & $\begin{array}{c}0.284 * * \\
* \\
{[9.38]}\end{array}$ \\
\hline Leverage, $t-1$ & $\begin{array}{c}0.018 * * \\
*\end{array}$ & $\begin{array}{c}0.018^{* *} \\
*\end{array}$ & $0.018^{* *}$ & $0.018^{* *}$ & $\begin{array}{c}0.015^{* *} \\
*\end{array}$ & $\begin{array}{c}0.015^{* *} \\
*\end{array}$ \\
\hline Loans/Assets, $t-1$ & $\begin{array}{c}{[3.56]} \\
0.092 \\
{[0.52]}\end{array}$ & $\begin{array}{c}{[3.55]} \\
0.118 \\
{[0.67]}\end{array}$ & $\begin{array}{c}{[2.27]} \\
0.445 \\
{[1.63]}\end{array}$ & $\begin{array}{c}{[2.27]} \\
0.431 \\
{[1.58]}\end{array}$ & $\begin{array}{l}{[2.61]} \\
-0.210 \\
{[-1.12]}\end{array}$ & $\begin{array}{l}{[2.60]} \\
-0.155 \\
{[-0.82]}\end{array}$ \\
\hline Deposits/Assets, $t-1$ & $\begin{array}{c}2.943 * * \\
*\end{array}$ & $\begin{array}{c}2.969^{* * *} \\
*\end{array}$ & 1.435 & 1.420 & $\begin{array}{c}4.212^{* *} \\
*\end{array}$ & $\begin{array}{c}4.266^{* *} \\
*\end{array}$ \\
\hline & $\begin{array}{c}{[4.73]} \\
-\end{array}$ & $\begin{array}{c}{[4.76]} \\
-\end{array}$ & {$[1.62]$} & {$[1.60]$} & $\begin{array}{c}{[4.50]} \\
-\end{array}$ & $\begin{array}{c}{[4.54]} \\
-\end{array}$ \\
\hline Deposits/Liability, $t-1$ & $\begin{array}{c}2.231 * * \\
*\end{array}$ & $\begin{array}{c}2.229 * * \\
*\end{array}$ & -0.966 & -0.965 & $\begin{array}{c}3.352^{* *} \\
*\end{array}$ & $\begin{array}{c}3.350^{* * *} \\
*\end{array}$ \\
\hline Constant & $\begin{array}{c}{[-4.18]} \\
-0.179 \\
{[-0.89]}\end{array}$ & $\begin{array}{c}{[-4.17]} \\
-0.207 \\
{[-1.00]}\end{array}$ & $\begin{array}{l}{[-1.41]} \\
-0.214 \\
{[-0.77]}\end{array}$ & $\begin{array}{l}{[-1.41]} \\
-0.194 \\
{[-0.67]}\end{array}$ & $\begin{array}{l}{[-4.02]} \\
-0.231 \\
{[-0.81]}\end{array}$ & $\begin{array}{c}{[-4.01]} \\
-0.291 \\
{[-1.00]}\end{array}$ \\
\hline Number of Observations & 7,256 & 7,256 & 3,253 & 3,253 & 4,003 & 4,003 \\
\hline Numl & 962 & 962 & 48 & 481 & 481 & 481 \\
\hline $\operatorname{Adj}$ & 0.555 & 0.555 & 0.543 & 0.543 & 0.585 & 0.585 \\
\hline Bank Fixed Effects & YES & YES & YES & YES & YES & YES \\
\hline Year Fixed Effects & YES & YES & YES & YES & YES & YES \\
\hline
\end{tabular}


Table 2.7. Firm $Q$ and Technology Investment

This table reports the results of the regressions of bank Firm $Q$ on their technology investment measures (Tech Expense/Total Assets, and Tech Expense/Loans \& Deposits) in the previous year in the sample from 2000-2017. The standard errors are clustered at the firm level. $t$-statistics based on robust standard errors are in brackets. Significance at the $1 \%, 5 \%$, or $10 \%$ levels are presented as*, **, or ***, respectively. All of the variables are defined in Table A1 in the appendix. Since lagged variables are included in regressions, firms with fewer than two consecutive years of technology expenses and total assets information are excluded. The variables have been winsorized at the $1 \%$ and $99 \%$ tails of the distributions to avoid the influence of extreme observations.

\begin{tabular}{|c|c|c|c|c|c|c|}
\hline Variables & $\begin{array}{c}\text { (1) } \\
\text { All } \\
\text { Banks } \\
\end{array}$ & $\begin{array}{c}(2) \\
\text { All } \\
\text { Banks } \\
\end{array}$ & $\begin{array}{c}(3) \\
\text { Small } \\
\text { Banks } \\
\end{array}$ & $\begin{array}{c}(4) \\
\text { Small } \\
\text { Banks } \\
\end{array}$ & $\begin{array}{c}(5) \\
\text { Large } \\
\text { Banks } \\
\end{array}$ & $\begin{array}{c}(6) \\
\text { Large } \\
\text { Banks } \\
\end{array}$ \\
\hline Tech Expense/Total Assets, $t-1$ & $\begin{array}{c}0.016 \\
{[1.27]}\end{array}$ & & $\begin{array}{l}-0.010 \\
{[-0.69]}\end{array}$ & & $\begin{array}{c}0.038 * \\
{[1.92]}\end{array}$ & \\
\hline Tech Expense/Loans \& Deposits, $t-1$ & & $\begin{array}{l}0.020 \\
{[1.13]}\end{array}$ & & $\begin{array}{l}-0.016 \\
{[-0.76]}\end{array}$ & & $\begin{array}{c}0.050 * \\
{[1.85]}\end{array}$ \\
\hline Log Market Capitalization, $t-1$ & $\begin{array}{c}0.019 * * \\
* \\
{[7.83]}\end{array}$ & $\begin{array}{c}0.019 * * \\
* \\
{[7.81]}\end{array}$ & $\begin{array}{c}0.017 * * \\
* \\
{[4.36]}\end{array}$ & $\begin{array}{c}0.017 * * \\
* \\
{[4.37]}\end{array}$ & $\begin{array}{c}0.020 * * \\
* \\
{[6.74]}\end{array}$ & $\begin{array}{c}0.020 * * \\
* \\
{[6.71]}\end{array}$ \\
\hline Leverage, $t$ - 1 & $0.001 * *$ & $0.001 * *$ & $\begin{array}{c}0.001 * * \\
*\end{array}$ & $\begin{array}{c}0.001 * * \\
*\end{array}$ & 0.000 & 0.000 \\
\hline Loans/Assets, $t-1$ & $\begin{array}{c}{[2.46]} \\
0.005 \\
{[0.32]}\end{array}$ & $\begin{array}{l}{[2.46]} \\
0.007 \\
{[0.44]}\end{array}$ & $\begin{array}{c}{[2.79]} \\
0.033 \\
{[1.35]}\end{array}$ & $\begin{array}{c}{[2.79]} \\
0.032 \\
{[1.30]}\end{array}$ & $\begin{array}{l}{[0.78]} \\
-0.022 \\
{[-1.28]}\end{array}$ & $\begin{array}{l}{[0.77]} \\
-0.017 \\
{[-1.02]}\end{array}$ \\
\hline Deposits/Assets, $t-1$ & $\begin{array}{c}0.235 * * \\
* \\
{[4.21]} \\
-\end{array}$ & $\begin{array}{c}0.237 * * \\
* \\
{[4.24]} \\
-\end{array}$ & $\begin{array}{l}0.094 \\
{[1.24]}\end{array}$ & $\begin{array}{l}0.093 \\
{[1.22]}\end{array}$ & $\begin{array}{c}0.352^{* *} \\
* \\
{[4.42]} \\
-\end{array}$ & $\begin{array}{c}0.357 * * \\
* \\
{[4.48]} \\
-\end{array}$ \\
\hline Deposits/Liability, $t-1$ & $\begin{array}{c}0.163 * * \\
* \\
{[-3.25]}\end{array}$ & $\begin{array}{c}0.163 * * \\
* \\
{[-3.25]}\end{array}$ & $\begin{array}{l}-0.045 \\
{[-0.71]}\end{array}$ & $\begin{array}{l}-0.045 \\
{[-0.71]}\end{array}$ & $\begin{array}{c}0.266 * * \\
* \\
{[-3.63]}\end{array}$ & $\begin{array}{c}0.267 * * \\
* \\
{[-3.63]}\end{array}$ \\
\hline Constant & $\begin{array}{c}0.909 * * \\
* \\
{[46.18]}\end{array}$ & $\begin{array}{c}0.907 * * \\
* \\
{[44.57]}\end{array}$ & $\begin{array}{c}0.893 * * \\
* \\
{[35.76]}\end{array}$ & $\begin{array}{c}0.895 * * \\
* \\
{[34.64]}\end{array}$ & $\begin{array}{c}0.922 * * \\
* \\
{[32.73]}\end{array}$ & $\begin{array}{c}0.917 * * \\
* \\
{[31.59]}\end{array}$ \\
\hline $\begin{array}{l}\text { Number of Observations } \\
\text { Number of Banks } \\
\text { Adjusted } R \text {-squared } \\
\text { Bank Fixed Effects } \\
\text { Year Fixed Effects }\end{array}$ & $\begin{array}{l}7,285 \\
963 \\
0.560 \\
\text { YES } \\
\text { YES }\end{array}$ & $\begin{array}{c}7,285 \\
963 \\
0.560 \\
\text { YES } \\
\text { YES } \\
\end{array}$ & $\begin{array}{l}3,265 \\
481 \\
0.573 \\
\text { YES } \\
\text { YES }\end{array}$ & $\begin{array}{c}3,265 \\
481 \\
0.573 \\
\text { YES } \\
\text { YES }\end{array}$ & $\begin{array}{l}4,020 \\
482 \\
0.574 \\
\text { YES } \\
\text { YES }\end{array}$ & $\begin{array}{c}4,020 \\
482 \\
0.573 \\
\text { YES } \\
\text { YES }\end{array}$ \\
\hline
\end{tabular}


Table 2.8. Robustness Checks

This table presents the results of the robustness checks.

Panels A, B and C keep firm-year observations whose technology expenses exceed \$1 million, $\$ 3$ million and $\$ 5$ million, respectively, and reports the results of regressions of ROA, ROE, Profit Margin, Asset Turnover, market-to-book, and $Q$ on their technology spending measures in the previous year.

Panel D drops too-big-to-fail banks from large banks and reports the results of regressions of ROA, ROE, Profit Margin, Asset Turnover, market-to-book, and $Q$ on their technology spending measures in the previous year.

Panel $\mathrm{E}$ and $\mathrm{F}$ includes large banks and reports the results of regressions of ROA and ROE on their previous one- to five-year technology expenses related to total assets and to total loans and deposits, respectively.

Each cell is a separate regression for the dependent variable indicated in the column header and the independent variables indicated in the row label. The reported observations and firms are from regressions when the independent variable is the previous-year technology expense related to total assets. Control variables include the previous-year market cap, leverage, loans/assets, deposits/assets, and deposits/liability. The standard errors are clustered at the firm level. $t$-statistics based on robust standard errors are in brackets. Significance at the $1 \%, 5 \%$ or $10 \%$ levels are presented as*, $* *$, or $* * *$, respectively. All of the variables are defined in Table A1 in the appendix. Since lagged variables are included in regressions, firms with fewer than two consecutive years of technology expenses and total assets information are excluded. The variables have been winsorized at the $1 \%$ and $99 \%$ tails of the distributions to avoid the influence of extreme observations.

Panel A. Banks Whose Technology Expense > \$1 Million

\begin{tabular}{|c|c|c|c|c|c|c|}
\hline Variables & $\begin{array}{c}(1) \\
\text { Return } \\
\text { on } \\
\text { Assets } \\
\end{array}$ & $\begin{array}{c}(2) \\
\text { Return } \\
\text { on } \\
\text { Equity } \\
\end{array}$ & $\begin{array}{c}\text { (3) } \\
\text { Profit } \\
\text { Margin }\end{array}$ & $\begin{array}{c}\text { (4) } \\
\text { Asset } \\
\text { Turnover }\end{array}$ & $\begin{array}{c}\text { (5) } \\
\text { Market- } \\
\text { to- } \\
\text { Book } \\
\end{array}$ & (6) \\
\hline \multirow{4}{*}{$\begin{array}{l}\text { Tech Expense/Total } \\
\text { Assets, } t-1 \\
\text { Tech Expense/Loans \& } \\
\text { Deposits, } t-1\end{array}$} & $0.751 *$ & $12.719 *$ & 10.545 & $1.855 * * *$ & $0.461 * *$ & $0.034 *$ \\
\hline & {$[1.84]$} & [1.79] & {$[1.01]$} & [4.53] & [2.32] & {$[1.94]$} \\
\hline & $1.009 *$ & 16.812 & 13.329 & $2.644 * * *$ & $0.553 * *$ & $0.043^{*}$ \\
\hline & {$[1.74]$} & [1.63] & {$[0.90]$} & {$[4.72]$} & {$[2.02]$} & {$[1.77]$} \\
\hline Number of Observations & 4,121 & 4,121 & 4,121 & 4,563 & 4,543 & 4,560 \\
\hline Number of Banks & 626 & 626 & 626 & 666 & 664 & 666 \\
\hline Control Variables & YES & YES & YES & YES & YES & YES \\
\hline Bank Fixed Effects & YES & YES & YES & YES & YES & YES \\
\hline Year Fixed Effects & YES & YES & YES & YES & YES & YES \\
\hline
\end{tabular}


Panel B. Banks Whose Technology Expense > \$3 Million

(1) (2) (3)

Return

Variables
Return Profit Asset on
Assets

Asset Market- $\begin{array}{llllll}1.270 * * * & 21.195 * * * & 16.237 & 2.504 * * * & 0.606 * * & 0.050 *\end{array}$

Assets, $t-1$

Tech Expense/Loans \&

Deposits, $t-1$

$$
\left[\begin{array}{llllll}
{[2.62]} & {[2.96]} & {[1.32]} & {[3.88]} & {[2.26]} & {[1.92]}
\end{array}\right.
$$

$\begin{array}{lllll}1.734 * * * 28.852 * * * & 21.514 & 3.530 * * * & 0.666 * & 0.061 *\end{array}$

$\left[\begin{array}{llllll}{[2.73]} & {[3.08]} & {[1.32]} & {[4.12]} & {[1.86]} & {[1.73]}\end{array}\right.$

\begin{tabular}{lcccccc}
\hline Number of Observations & 1,926 & 1,926 & 1,926 & 2,203 & 2,194 & 2,203 \\
Number of Banks & 314 & 314 & 314 & 338 & 337 & 338 \\
Control Variables & YES & YES & YES & YES & YES & YES \\
Bank Fixed Effects & YES & YES & YES & YES & YES & YES \\
Year Fixed Effects & YES & YES & YES & YES & YES & YES \\
\hline
\end{tabular}

Panel C. Banks Whose Technology Expense > \$5 Million

\begin{tabular}{|c|c|c|c|c|c|c|}
\hline Variables & $\begin{array}{c}\text { (1) } \\
\text { Return } \\
\text { on } \\
\text { Assets }\end{array}$ & $\begin{array}{c}\text { (2) } \\
\text { Return } \\
\text { on Equity }\end{array}$ & $\begin{array}{c}\text { (3) } \\
\text { Profit } \\
\text { Margin }\end{array}$ & $\begin{array}{c}(4) \\
\text { Asset } \\
\text { Turnover }\end{array}$ & $\begin{array}{c}\text { (5) } \\
\text { Market- } \\
\text { to-Book }\end{array}$ & (6) \\
\hline $\begin{array}{l}\text { Tech Expense/Total } \\
\text { Assets, } t-1\end{array}$ & $\begin{array}{c}1.384 * * * \\
{[2.76]}\end{array}$ & $\begin{array}{c}17.150 * * * \\
{[2.63]}\end{array}$ & $\begin{array}{l}19.159 \\
{[1.62]}\end{array}$ & $\begin{array}{c}2.358 * * * \\
{[4.29]}\end{array}$ & $\begin{array}{c}0.662 * * \\
{[2.42]}\end{array}$ & $\begin{array}{c}0.058 * * \\
{[2.20]}\end{array}$ \\
\hline $\begin{array}{l}\text { Tech Expense/Loans \& } \\
\text { Deposits, } t-1\end{array}$ & $\begin{array}{c}1.859 * * * \\
{[2.93]}\end{array}$ & $\begin{array}{c}23.165 * * * \\
{[2.74]}\end{array}$ & $\begin{array}{l}24.698 \\
{[1.65]}\end{array}$ & $\begin{array}{c}3.241 * * * \\
{[4.59]}\end{array}$ & $\begin{array}{l}0.706^{*} \\
{[1.92]}\end{array}$ & $\begin{array}{c}0.070 * * \\
{[1.98]}\end{array}$ \\
\hline $\begin{array}{l}\text { Number of Observations } \\
\text { Number of Banks } \\
\text { Control Variables } \\
\text { Bank Fixed Effects } \\
\text { Year Fixed Effects }\end{array}$ & $\begin{array}{l}1,309 \\
217 \\
\text { YES } \\
\text { YES } \\
\text { YES }\end{array}$ & $\begin{array}{l}1,309 \\
217 \\
\text { YES } \\
\text { YES } \\
\text { YES }\end{array}$ & $\begin{array}{l}1,309 \\
217 \\
\text { YES } \\
\text { YES } \\
\text { YES }\end{array}$ & $\begin{array}{l}1,492 \\
241 \\
\text { YES } \\
\text { YES } \\
\text { YES }\end{array}$ & $\begin{array}{l}1,487 \\
241 \\
\text { YES } \\
\text { YES } \\
\text { YES }\end{array}$ & $\begin{array}{l}1,492 \\
241 \\
\text { YES } \\
\text { YES } \\
\text { YES }\end{array}$ \\
\hline
\end{tabular}


Panel D. Large Banks with Too-big-to-fail Banks Excluded

\begin{tabular}{|c|c|c|c|c|c|c|}
\hline Variables & $\begin{array}{c}\text { (1) } \\
\text { Return } \\
\text { on } \\
\text { Assets }\end{array}$ & $\begin{array}{c}\text { (2) } \\
\text { Return } \\
\text { on Equity }\end{array}$ & $\begin{array}{c}\text { (3) } \\
\text { Profit } \\
\text { Margin }\end{array}$ & $\begin{array}{c}\text { (4) } \\
\text { Asset } \\
\text { Turnover }\end{array}$ & $\begin{array}{c}\text { (5) } \\
\text { Market- } \\
\text { to-Book }\end{array}$ & (6) \\
\hline $\begin{array}{l}\text { Tech Expense/Total } \\
\text { Assets, } t-1\end{array}$ & $\begin{array}{c}1.003 * * * \\
{[3.19]}\end{array}$ & $\begin{array}{c}17.535 * * * \\
{[3.36]}\end{array}$ & $\begin{array}{c}18.672 * * \\
{[2.45]}\end{array}$ & $\begin{array}{c}1.822 * * * \\
{[3.74]}\end{array}$ & $\begin{array}{c}0.455 * * \\
{[2.02]}\end{array}$ & $\begin{array}{l}0.030 \\
{[1.47]}\end{array}$ \\
\hline $\begin{array}{l}\text { Tech Expense/Loans \& } \\
\text { Deposits, } t-1\end{array}$ & $\begin{array}{c}1.403 * * * \\
{[3.19]}\end{array}$ & $\begin{array}{c}24.733 * * * \\
{[3.40]}\end{array}$ & $\begin{array}{c}24.602 * * \\
{[2.30]}\end{array}$ & $\begin{array}{c}2.769 * * * \\
{[4.06]}\end{array}$ & $\begin{array}{c}0.629 * * \\
{[2.00]}\end{array}$ & $\begin{array}{l}0.043 \\
{[1.51]}\end{array}$ \\
\hline $\begin{array}{l}\text { Number of Observations } \\
\text { Number of Banks } \\
\text { Control Variables } \\
\text { Bank Fixed Effects } \\
\text { Year Fixed Effects }\end{array}$ & $\begin{array}{l}3,395 \\
426 \\
\text { YES } \\
\text { YES } \\
\text { YES }\end{array}$ & $\begin{array}{l}3,395 \\
426 \\
\text { YES } \\
\text { YES } \\
\text { YES }\end{array}$ & $\begin{array}{l}3,395 \\
426 \\
\text { YES } \\
\text { YES } \\
\text { YES }\end{array}$ & $\begin{array}{c}3,787 \\
457 \\
\text { YES } \\
\text { YES } \\
\text { YES }\end{array}$ & $\begin{array}{l}3,770 \\
456 \\
\text { YES } \\
\text { YES } \\
\text { YES }\end{array}$ & $\begin{array}{c}3,787 \\
457 \\
\text { YES } \\
\text { YES } \\
\text { YES }\end{array}$ \\
\hline
\end{tabular}

Panel E. Large Banks without Mergers and Acquisitions Activities

\begin{tabular}{|c|c|c|c|c|c|c|}
\hline Variables & $\begin{array}{c}(1) \\
\text { Return } \\
\text { on } \\
\text { Assets } \\
\end{array}$ & $\begin{array}{c}(2) \\
\text { Return } \\
\text { on Equity }\end{array}$ & $\begin{array}{c}(3) \\
\text { Profit } \\
\text { Margin }\end{array}$ & $\begin{array}{c}\text { (4) } \\
\text { Asset } \\
\text { Turnover }\end{array}$ & $\begin{array}{c}\text { (5) } \\
\text { Market- } \\
\text { to-Book }\end{array}$ & $\begin{array}{l}\text { (6) } \\
Q\end{array}$ \\
\hline $\begin{array}{l}\text { Tech Expense/Total } \\
\text { Assets, } t-1\end{array}$ & $3.175^{* * *} *$ & $57.759 * * *$ & $61.684 * * *$ & $3.162 * * *$ & 0.785 & 0.049 \\
\hline Tech Expense/Loans \& & $\begin{array}{c}{[4.18]} \\
4.195^{* * *}\end{array}$ & $\begin{array}{c}{[4.16]} \\
78.290 * * *\end{array}$ & $\begin{array}{c}{[3.18]} \\
77.933 * * *\end{array}$ & $\begin{array}{c}{[3.24]} \\
4.478 * * *\end{array}$ & $\begin{array}{l}{[1.60]} \\
0.881\end{array}$ & $\begin{array}{l}{[1.20]} \\
0.055\end{array}$ \\
\hline & [3.94] & [3.97] & [2.83] & [3.43] & [1.27] & [0.96] \\
\hline Number of Observations & 923 & 923 & 923 & 1,054 & 1,041 & 1,054 \\
\hline Number of Banks & 142 & 142 & 142 & 155 & 154 & 155 \\
\hline Control Variables & YES & YES & YES & YES & YES & YES \\
\hline Bank Fixed Effects & YES & YES & YES & YES & YES & YES \\
\hline Year Fixed Effects & YES & YES & YES & YES & YES & YES \\
\hline
\end{tabular}


Panel F. Return on Assets and Multi-lagged Technology Investment

\begin{tabular}{lccccc}
\hline Variables & $(1)$ & $(2)$ & $(3)$ & $(4)$ & $(5)$ \\
& $m=1$ & $m=2$ & $m=3$ & $m=4$ & $m=5$ \\
$\begin{array}{l}\text { Tech Expense/Total } \\
\text { Assets, } t-m\end{array}$ & $0.927 * * *$ & $1.222 * * *$ & $1.253 * * *$ & $1.366 * * *$ & 0.547 \\
& {$[3.10]$} & {$[3.90]$} & {$[3.51]$} & {$[3.63]$} & {$[1.41]$} \\
Tech Expense/Loans \& & $1.317 * * *$ & $1.733 * * *$ & $1.714 * * *$ & $1.839 * * *$ & 0.700 \\
Deposits, $t-m$ & {$[3.21]$} & {$[4.08]$} & {$[3.66]$} & {$[3.67]$} & {$[1.30]$} \\
& & & & & \\
\hline Number of Observations & 3,592 & 3,193 & 2,810 & 2,424 & 2,069 \\
Number of Banks & 449 & 432 & 414 & 380 & 341 \\
Control Variables & YES & YES & YES & YES & YES \\
Bank Fixed Effects & YES & YES & YES & YES & YES \\
Year Fixed Effects & YES & YES & YES & YES & YES \\
\hline
\end{tabular}

Panel G. Return on Equity and Multi-lagged Technology Investment

\begin{tabular}{lccccc}
\hline & $(1)$ & $(2)$ & $(3)$ & $(4)$ & $(5)$ \\
Variables & $m=1$ & $m=2$ & $m=3$ & $m=4$ & $m=5$ \\
\hline & & & & & \\
Tech Expense/Total & $15.752 * * *$ & $18.364 * * *$ & $19.713^{* * * *}$ & $19.066^{* * *}$ & 7.081 \\
Assets, $t-m$ & {$[3.25]$} & {$[4.10]$} & {$[3.87]$} & {$[3.81]$} & {$[1.20]$} \\
& & & & & \\
Tech Expense/Loans \& & $22.112^{* * *}$ & $26.056^{* * *}$ & $26.925 * * *$ & $25.880 * * *$ & 8.302 \\
Deposits, $t-m$ & {$[3.32]$} & {$[4.28]$} & {$[4.06]$} & {$[3.87]$} & {$[1.01]$} \\
& & & & & \\
\hline $\begin{array}{l}\text { Number of Observations } \\
\text { Number of Banks }\end{array}$ & 3,592 & 3,193 & 2,810 & 2,424 & 2,069 \\
Control Variables & 449 & 432 & 414 & 380 & 341 \\
Bank Fixed Effects & YES & YES & YES & YES & YES \\
Year Fixed Effects & YES & YES & YES & YES & YES \\
\hline
\end{tabular}



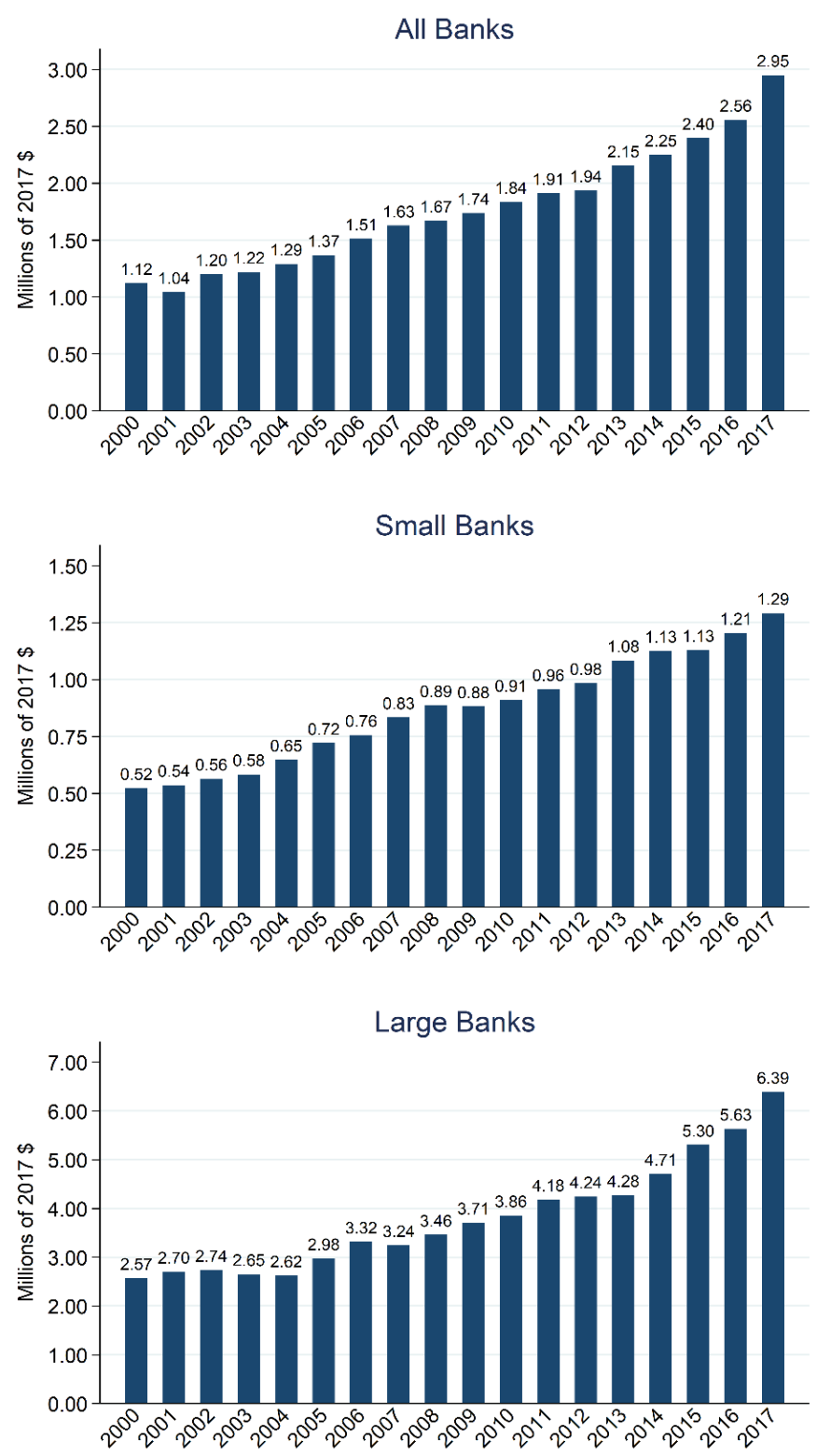

Figure 2.1. Bank Technology Spending Trends

This figure illustrates the trends of technology and communication expenses (based on median) of U.S listed commercial banks in the sample from 2000-2017. Technology and communication expense is converted to 2017 dollars using the GDP deflator. All of the variables are defined in Table A1 in the appendix. Since lagged variables are included in regressions, firms with fewer than two consecutive years of technology expenses and total assets information are excluded. The variables have been winsorized at the $1 \%$ and $99 \%$ tails of the distributions to avoid the influence of extreme observations. 

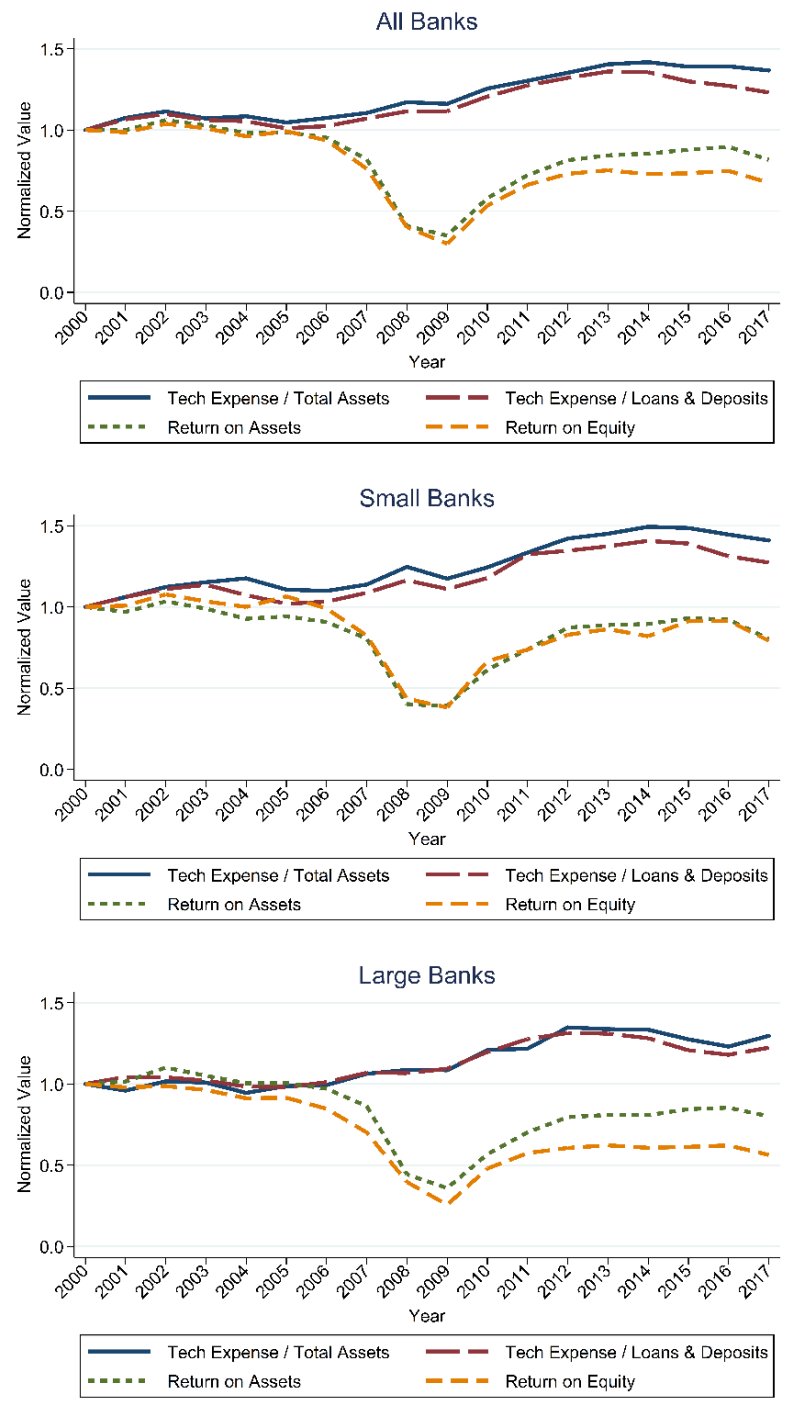

Figure 2.2. Performance and Technology Investment Over Time

This figure illustrates the medians of bank performance measures (Return on Assets and Return on Equity) and technology investment measures (Tech Expense/Total Assets and Tech Expense/Loans \& Deposits) of U.S listed commercial banks in the sample from 20002017. All of the values are normalized to equal one in the year 2000. All of the variables are defined in Table A1 in the appendix. Since lagged variables are included in regressions, firms with fewer than two consecutive years of technology expenses and total assets information are excluded. The variables have been winsorized at the $1 \%$ and $99 \%$ tails of the distributions to avoid the influence of extreme observations. 

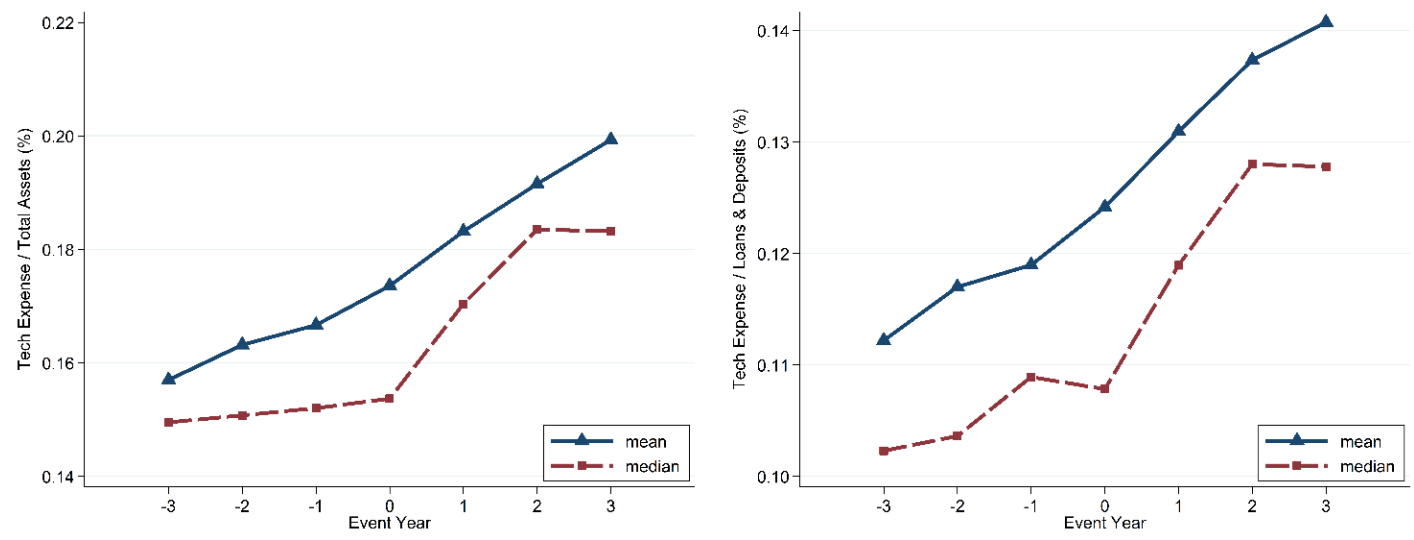

Figure 2.3. Technology Investment Around Negative Performance Shocks

This figure illustrates the means and the medians of the bank technology investment measures (Tech Expense/Total Assets, and Tech Expense/Loans \& Deposits) before and after three years of the bank negative performance shocks. The performance shock is defined as banks' Return on Assets are one standard deviation less than that in the previous year. All of the variables are defined in Table A1 in the appendix. Since lagged variables are included in regressions, firms with fewer than two consecutive years of technology expenses and total assets information are excluded. The variables have been winsorized at the $1 \%$ and $99 \%$ tails of the distributions to avoid the influence of extreme observations. 
Appendix

Table A1. Definition of Variables

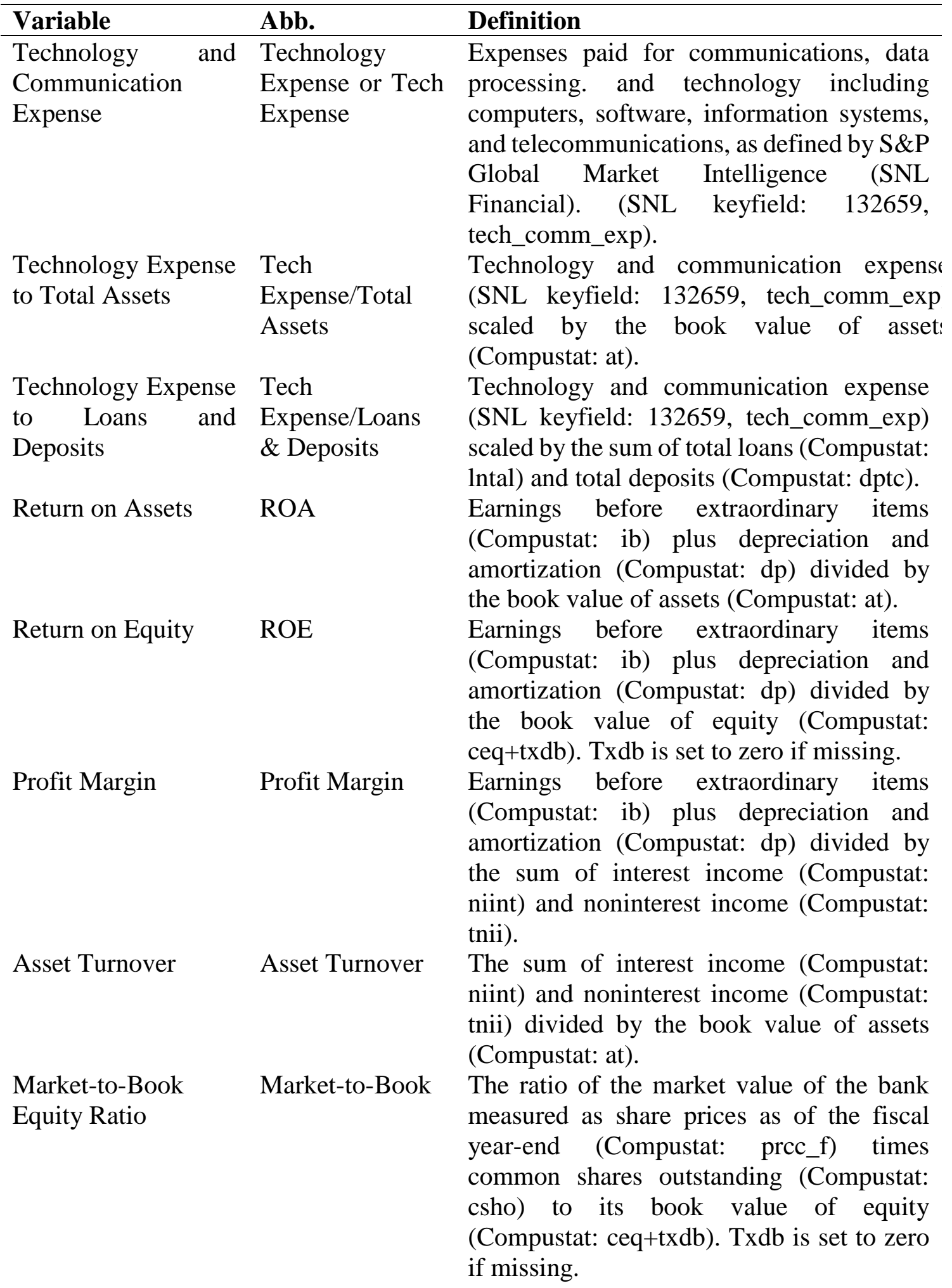




\begin{tabular}{|c|c|c|}
\hline Firm Value $Q$ & $Q$ & $\begin{array}{l}\text { The ratio of the market value of the bank } \\
\text { measured as share prices as of the fiscal } \\
\text { year-end (Compustat: prcc_f) times } \\
\text { common shares outstanding (Compustat: } \\
\text { csho) plus total assets (Compustat: at) } \\
\text { minus the book value of equity (Compustat: } \\
\text { ceq+txdb) to its total assets (Compustat: at). } \\
\text { Txdb is set to zero if missing. }\end{array}$ \\
\hline $\begin{array}{l}\text { Market } \\
\text { Capitalization }\end{array}$ & MktCap (Size) & $\begin{array}{l}\text { Share prices as of the fiscal year-end } \\
\text { (Compustat: prcc_f) times common shares } \\
\text { outstanding (Compustat: csho). }\end{array}$ \\
\hline Leverage & Leverage & $\begin{array}{l}\text { The ratio of total assets (Compustat: at) to } \\
\text { the book value of equity (Compustat: } \\
\text { ceq }+t x d b) \text {. Txdb is set to zero if missing. }\end{array}$ \\
\hline $\begin{array}{l}\text { Total Loans/Total } \\
\text { Assets }\end{array}$ & Loans/Assets & $\begin{array}{l}\text { The ratio of the banks' total loans } \\
\text { (Compustat: lntal) to total assets } \\
\text { (Compustat: at). }\end{array}$ \\
\hline $\begin{array}{l}\text { Total Deposits/Total } \\
\text { Assets }\end{array}$ & ts/Assets & $\begin{array}{l}\text { The ratio of the banks' total deposits } \\
\text { (Compustat: dptc) to total assets } \\
\text { (Compustat: at). }\end{array}$ \\
\hline $\begin{array}{l}\text { Total Deposits/Total } \\
\text { Liability }\end{array}$ & $\begin{array}{l}\text { Deposits/Liabilit } \\
\text { y }\end{array}$ & $\begin{array}{l}\text { The ratio of the banks' total deposits } \\
\text { (Compustat: dptc) to total liabilities } \\
\text { (Compustat: } 1 \text { ). }\end{array}$ \\
\hline Gross Total Assets & GTA & $\begin{array}{l}\text { The sum of total assets (Compustat: at) and } \\
\text { provision for loan losses (Compustat: pclc). } \\
\text { pclc is set to zero if missing. }\end{array}$ \\
\hline Small Banks & Small Banks & $\begin{array}{l}\text { Banks whose time-series average GTA are } \\
\text { up to } \$ 1 \text { billion in } 2017 \text { dollars. }\end{array}$ \\
\hline Large Banks & Large Banks & $\begin{array}{l}\text { Banks whose time-series average GTA } \\
\text { exceed } \$ 1 \text { billion in } 2017 \text { dollars. }\end{array}$ \\
\hline $\begin{array}{l}\text { Too-big-to-fail } \\
\text { banks }\end{array}$ & TBTF Banks & $\begin{array}{l}\text { Banks whose time-series average GTA } \\
\text { exceed } \$ 50 \text { billion in } 2017 \text { dollars. }\end{array}$ \\
\hline
\end{tabular}


Table A2. Discussion on Technology and Communication Expense

The technology and communication expense include expenses paid for communications, such as telephone and fax usage charges, internet data plans, and mobile phone and internet plans, data processing and technology including computers, wire services, modems, routers, and switches, as well as software purchases and subscriptions to cloud-based services. The variable is primarily constructed based on U.S. GAAP Standard FAS No. 86. Some typical examples are as follows:

\begin{tabular}{|c|c|c|c|c|c|}
\hline \multirow{3}{*}{$\begin{array}{l}\text { Bank } \\
\text { Citigroup }\end{array}$} & \multirow{3}{*}{$\begin{array}{l}\text { Ticker } \\
\text { C }\end{array}$} & \multirow{2}{*}{$\begin{array}{l}\text { Technolo } \\
\text { gy } \\
\text { Expense } \\
(\$ 000)\end{array}$} & \multirow[b]{2}{*}{ Decomposition } & & \multirow[b]{2}{*}{$\begin{array}{l}\text { Documents } \\
\text { (Sources) }\end{array}$} \\
\hline & & & & & \\
\hline & & $\begin{array}{l}\$ 6,581,0 \\
00\end{array}$ & $\begin{array}{l}\text { Technology/communic } \\
\text { ation }\end{array}$ & $\begin{array}{l}\$ 6,581,0 \\
00\end{array}$ & $\begin{array}{l}12 / 31 / 2015 \\
10-\mathrm{K}\end{array}$ \\
\hline \multirow{2}{*}{$\begin{array}{l}\text { Bank of } \\
\text { America }\end{array}$} & \multirow{2}{*}{ BAC } & \multirow{2}{*}{$\begin{array}{l}\$ 3,938,0 \\
00\end{array}$} & Telecommunications & $\$ 823,000$ & $\begin{array}{l}12 / 31 / 2015 \\
10-\mathrm{K}\end{array}$ \\
\hline & & & Data processing & $\begin{array}{l}\$ 3,115,0 \\
00\end{array}$ & $\begin{array}{l}12 / 31 / 2015 \\
10-\mathrm{K}\end{array}$ \\
\hline \multirow{2}{*}{$\begin{array}{l}\text { First } \\
\text { Citizens } \\
\text { BancShares } \\
\text {, Inc. }\end{array}$} & \multirow{2}{*}{$\begin{array}{l}\mathrm{FCNC} \\
\mathrm{A}\end{array}$} & \multirow[t]{2}{*}{$\$ 114,896$} & $\begin{array}{l}\text { Tech \& } \\
\text { Communications } \\
\text { Expense }\end{array}$ & $\$ 114,896$ & $\begin{array}{l}\text { Bank } \\
\text { Regulatory } \\
\text { Filings } \\
\end{array}$ \\
\hline & & & Telecommunications & $\$ 14,406$ & $\begin{array}{l}12 / 31 / 2015 \\
10-\mathrm{K}\end{array}$ \\
\hline \multirow{4}{*}{$\begin{array}{l}\text { Community } \\
\text { First } \\
\text { Bancorp, } \\
\text { Inc. }\end{array}$} & \multirow{4}{*}{ CMFP } & \multirow{4}{*}{$\$ 399$} & Data processing & $\$ 162,616$ & $\begin{array}{l}12 / 31 / 2015 \\
10-\mathrm{K}\end{array}$ \\
\hline & & & Telephone & $\$ 59,150$ & $\begin{array}{l}12 / 31 / 2015 \\
10-\mathrm{K}\end{array}$ \\
\hline & & & Internet banking & $\$ 87,643$ & $\begin{array}{l}12 / 31 / 2015 \\
10-\mathrm{K}\end{array}$ \\
\hline & & & ATM expenses & $\$ 89,771$ & $\begin{array}{l}12 / 31 / 2015 \\
10-\mathrm{K}\end{array}$ \\
\hline \multirow{3}{*}{$\begin{array}{l}\text { Guaranty } \\
\text { Federal } \\
\text { Bancshares } \\
\text {, Inc. }\end{array}$} & \multirow{3}{*}{ GFED } & \multirow{3}{*}{$\$ 1,171$} & Data processing & $\$ 790,928$ & $\begin{array}{l}12 / 31 / 2015 \\
10-\mathrm{K}\end{array}$ \\
\hline & & & Telephone & $\$ 141,674$ & $\begin{array}{l}12 / 31 / 2015 \\
10-\mathrm{K}\end{array}$ \\
\hline & & & ATM expense & $\$ 238,744$ & $\begin{array}{l}12 / 31 / 2015 \\
10-\mathrm{K}\end{array}$ \\
\hline \multirow{3}{*}{$\begin{array}{l}\text { Home } \\
\text { BancShares } \\
\text {, Inc. }\end{array}$} & \multirow{3}{*}{$\begin{array}{l}\mathrm{HOM} \\
\mathrm{B}\end{array}$} & \multirow{3}{*}{$\$ 17,857$} & $\begin{array}{l}\text { Data processing } \\
\text { expense }\end{array}$ & $\$ 10,774$ & $\begin{array}{l}12 / 31 / 2015 \\
10-\mathrm{K}\end{array}$ \\
\hline & & & $\begin{array}{l}\text { Electronic banking } \\
\text { expense }\end{array}$ & $\$ 5,166$ & $\begin{array}{l}12 / 31 / 2015 \\
10-\mathrm{K}\end{array}$ \\
\hline & & & Telephone & $\$ 1,917$ & $\begin{array}{l}12 / 31 / 2015 \\
10-\mathrm{K}\end{array}$ \\
\hline
\end{tabular}




\begin{tabular}{|c|c|c|c|c|c|}
\hline \multirow{2}{*}{$\begin{array}{l}\text { Pandora } \\
\text { Bancshares } \\
\text {, Inc. }\end{array}$} & \multirow[t]{2}{*}{ PDRB } & \multirow[t]{2}{*}{$\$ 736$} & $\begin{array}{l}\text { Tech \& } \\
\text { Communications } \\
\text { Expense }\end{array}$ & $\$ 736$ & \multirow{2}{*}{$\begin{array}{l}\text { Bank } \\
\text { Regulatory } \\
\text { Filings } \\
12 / 31 / 2015 \\
10-\mathrm{K}\end{array}$} \\
\hline & & & Data Processing & $\$ 505$ & \\
\hline $\begin{array}{l}\text { Webster } \\
\text { Financial } \\
\text { Corporatio } \\
\mathrm{n}\end{array}$ & WBS & $\$ 34,639$ & $\begin{array}{l}\text { Tech \& } \\
\text { Communications } \\
\text { Expense }\end{array}$ & $\$ 34,639$ & $\begin{array}{l}\text { Bank } \\
\text { Regulatory } \\
\text { Filings }\end{array}$ \\
\hline $\begin{array}{l}\text { First } \\
\text { Farmers } \\
\text { Financial } \\
\text { Corporatio } \\
\mathrm{n}\end{array}$ & FFMR & $\$ 2,303$ & $\begin{array}{l}\text { Tech \& } \\
\text { Communications } \\
\text { Expense }\end{array}$ & $\$ 2,303$ & $\begin{array}{l}\text { Bank } \\
\text { Regulatory } \\
\text { Filings }\end{array}$ \\
\hline
\end{tabular}



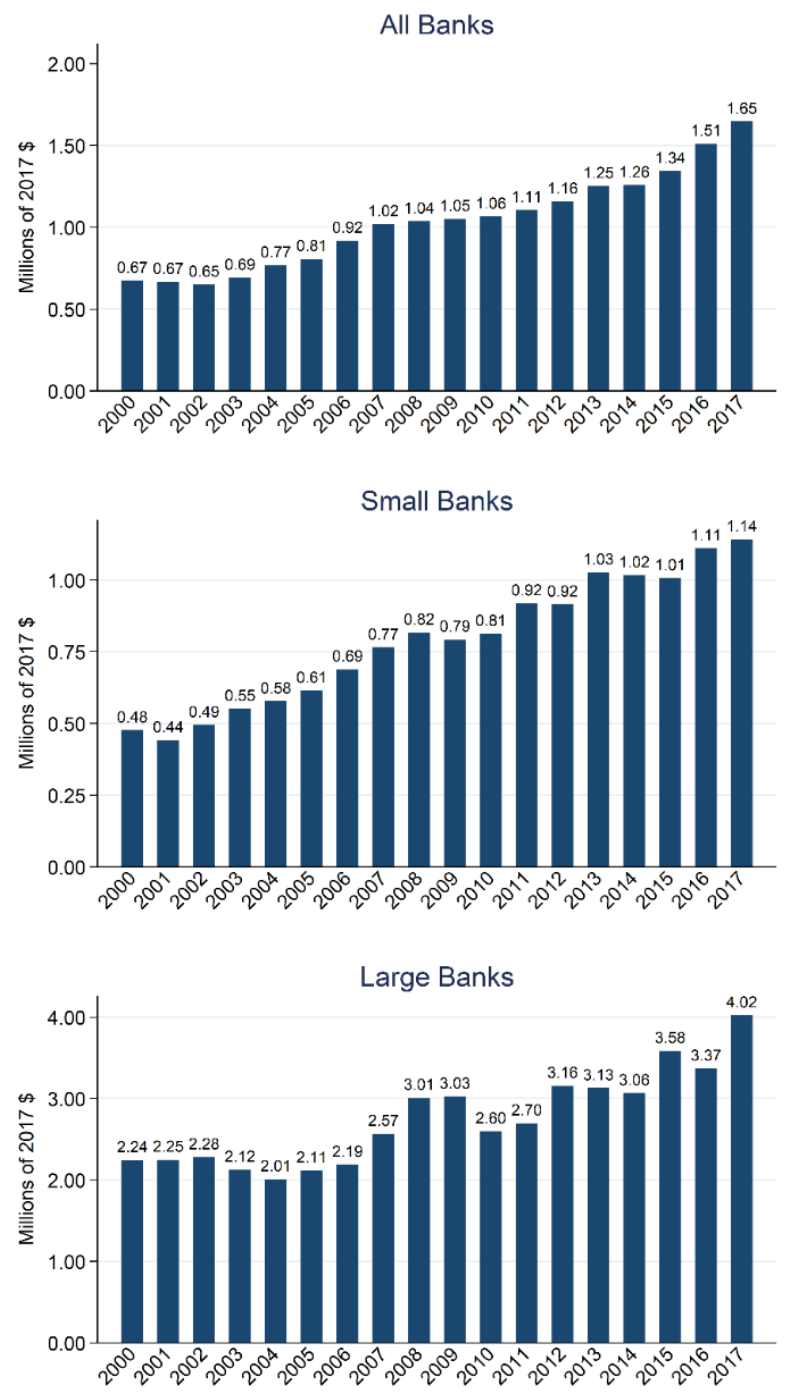

Figure B1. Bank Technology Spending Trends of Non-M\&A Banks

This figure illustrates the trends of technology and communication expenses (based on median) of U.S listed commercial banks without mergers and acquisitions activities (NonM\&A banks) in the sample from 2000-2017. Technology and communication expense is converted to 2017 dollars using the GDP deflator. All of the variables are defined in Table A1 in the appendix. Since lagged variables are included in regressions, firms with fewer than two consecutive years of technology expenses and total assets information are excluded. The variables have been winsorized at the $1 \%$ and $99 \%$ tails of the distributions to avoid the influence of extreme observations. 


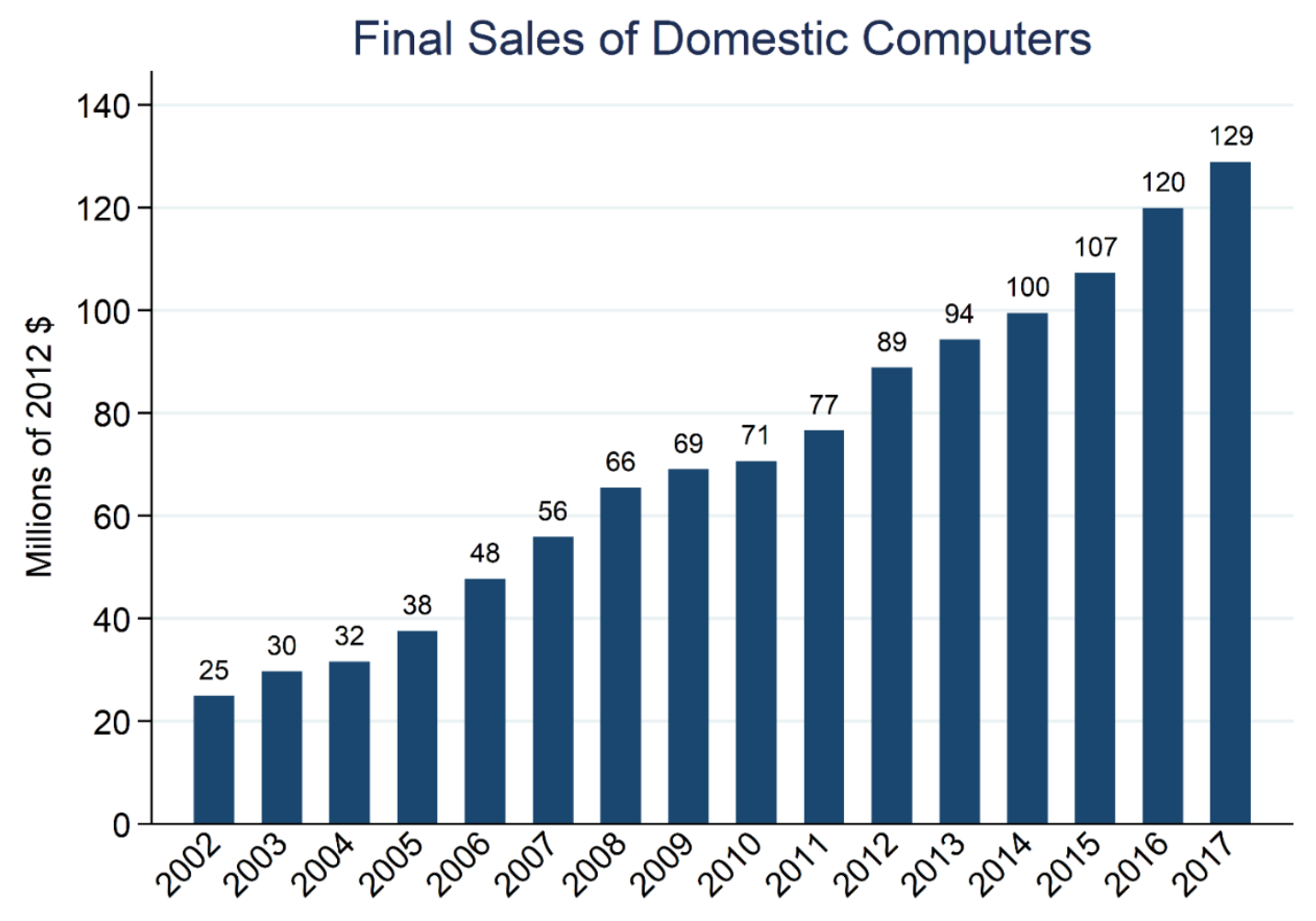

Figure B2. Final Sales of Domestic Computers

This figure illustrates the trends of Final Sales of Domestic Computers in the United States (in Billions of 2012 dollars) from the National Income and Product Accounts (Table 9.2U). 

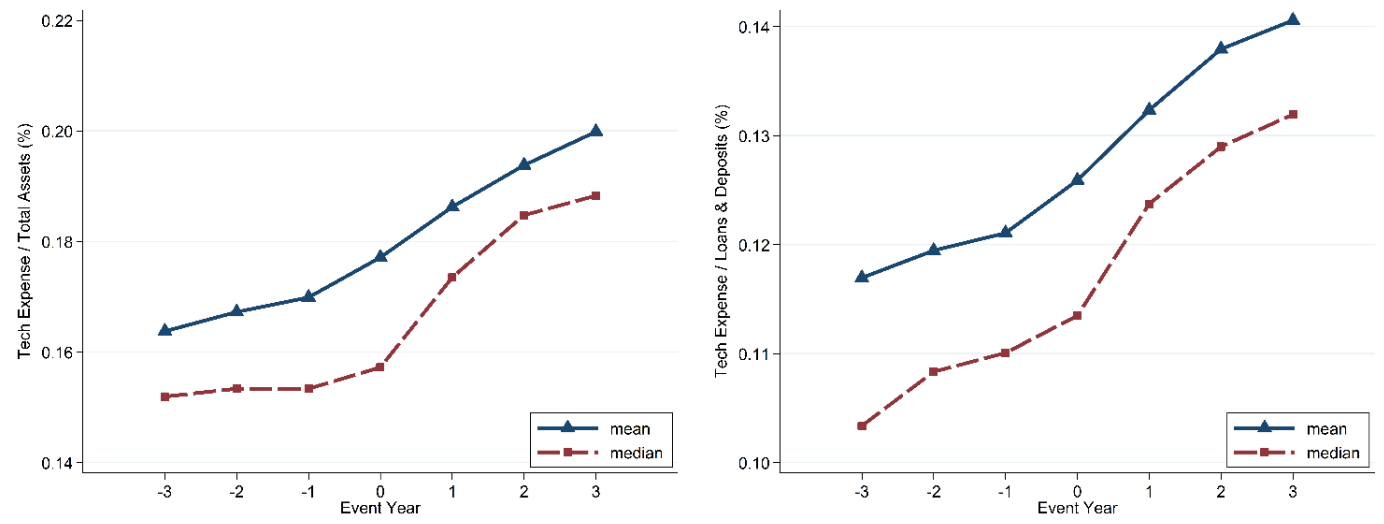

Figure B3. Technology Investment Around Negative Performance Shocks

This figure illustrates the means and the medians of the bank technology investment measures (Tech Expense/Total Assets, and Tech Expense/Loans \& Deposits) before and after three years of the bank negative performance shocks. The performance shock is defined as banks' Return on Equity are one standard deviation less than that in the previous year. All of the variables are defined in Table A1 in the appendix. Since lagged variables are included in regressions, firms with fewer than two consecutive years of technology expenses and total assets information are excluded. The variables have been winsorized at the $1 \%$ and $99 \%$ tails of the distributions to avoid the influence of extreme observations. 


\section{Chapter III: Bank Technology: Productivity and Employment}

\subsection{Introduction}

Advances in technology have transformed many aspects of the production process in many industries (Brynjolfsson and McAfee, 2014; Autor, 2015; Ford, 2015). The banking industry, which is considered one of the most technology-intensive industries in the U.S. (see Triplett and Bosworth, 2006), has also been significantly affected by technology advances (e.g., Berger 2003; Greenwood and Scharfstein, 2013; Brynjolfsson and McAfee, 2014; Philippon, 2016). During the past decade, technology spending by U.S. banks experienceddramatic growth (see Figure 1). Technology has become a critical component in the production of banks and has revolutionized how financial institutions operate - from their customer services, banking process, "Know Your Customer" (KYC) activities, to business Application Programming Interface (API), and many others. ${ }^{1,2}$ Given the important impact of technology advances, it is interesting to examine to which extent technology investment contributes to bank productivity over the past decade.

\footnotetext{
${ }^{1}$ See an industrial report from EY, "Global banking outlook 2015: Transforming banking for the next generation technology reshaping banking”, and DeYoung (2010).

${ }^{2}$ For instance, Barclays Bank provides an innovative video banking service, Wells Fargo allows its customers to make cash withdrawals from automatic teller machines (ATMs) using smartphones rather than debit cards.
} 
Meanwhile, with the rapid development of new technology, many are worried about that technology adoption, and automation reduces or destroys jobs. ${ }^{3}$ This concern has drawn great attention among researchers and policymakers in recent years. There is a growing literature investigating whether and howthe use of new technology affect employment (e.g., Acemoglu and Autor, 2011; Charles, Hurst, and Notowidigdo, 2013; Fort, Pierce, and Schott, 2018). From a theoretical standpoint, technology investment can impede employment via its labor-saving effects (e.g., Goos, Manning, and Salomons, 2014; DeCanio, 2016). However, others argue that the effects related to technological innovation can overcompensate the displacement effect (Harrison et al., 2014). Thus, technology can enhance employment via its employment-stimulating effects. For example, Acemoglu and Restrepo (2018) suggest technology adoption can increase firm productivity and generate new tasks. The creation of new tasks and more complex version of existing tasks by adopting new technology will increase employment and labor share. To understand the overall effect of technology on employment, the countervailing force should be incorporated, and additional empirical evidence is needed.

In the banking industry, new bank technology creates new opportunities for business expansion, and generates new jobs or tasks. Some believe that a significant number of banking jobs will be lost due to technology adoption in the near future. For instance, a report from Citibank warns that about $30 \%$ of banking jobs are likely to be lost

\footnotetext{
${ }^{3}$ See an article from MIT Technology Review on June 12, 2013, "How technology is destroying jobs", and an article from $B B C$ News on 6 August 2015, "Will machines eventually take on every job?", among many others.
} 
from 2015 to $2025 .{ }^{4}$ Also, based on a static production function framework assuming constant elasticity of substitution, Martin-Oliver and Salas-Fumas (2008) suggest that an additional 1 million Euros IT investment in Spanish banks may be substituted for 25 employees. However, others argue that new technology can create more jobs in the banking industry. ${ }^{5}$ Bogliacino and Pianta (2010) and Harrison et al. (2014) suggest that a major driver for employment growth of firms are technology and innovation. Bessen (2015) and David (2015) show that one of the greatest technology inventions in the banking industry - automatic teller machines (ATMs) - does not eliminate the teller job. Instead, it increases teller-related jobs. ${ }^{6}$ While technology adoption is well documented to replace labors over the past few decades in agriculture and manufacturing industries (e.g., Rifkin, 1996; Acemoglu and Restrepo, 2017), little empirical evidence has been provided regarding the effect of technology on bank employment.

This paper examines the benefit of technology capital in bank production and investigates howthe use of technology affects employment in the U.S. banking industry. Technology is considered as an essential core competency and a key driving force for the future growth of banks. ${ }^{7}$ Because of the significance of technology innovations, which

\footnotetext{
${ }^{4}$ See an article from $C N N$ News on April 4, 2016, "30\% of bank jobs are under threat".

${ }^{5}$ See an article from The Guardian on August 18, 2015, "Technology has created more jobs than it has destroyed, says 140 years of data", and from U.S. News on December 7, 2015, "Machines reshape more jobs than they destroy".

${ }^{6}$ See an article from The Economist on June 15, 2011, titled “Are ATMs stealing jobs?”.

${ }^{7}$ See page 52 in the 2015 Annual Report of JPMorgan Chase \& Co.
} 
have rapidly changed the business environment for the U.S., it is interesting to examine the effect of bank technology on production and employment.

The questions of interest in the paper include: Does the expanded technology adoption indeed improve bank productivity? Would the contribution of technology investment be greater than its cost? Do the labor-saving effects or employment-stimulating effects play a greater role? In other words, while algorithms and machines replace a part of bank jobs, are the adoption of new technology creating new jobs quick than they destroy them? The answers to these questions are of great importance to practitioners, academics, and policymakers, as evidenced by academic research, extensive media coverage and industrial reports in recent years.

With a unique sample of U.S. listed commercial banks from S\&P Global Market Intelligence from 2000 to 2017 , the contribution of technology capital on bank production and the effect of technology spending on bank employment is examined. ${ }^{8}$ First, the parameters of a value-added bank production function correcting for endogenous input is estimated choices to understand the benefits banks receive from technology capital. The results show that the estimated parameters for technology capital are statistically significant in all four specifications, ranging from 0.065 to 0.112 . On average, technology inputs contribute about $12.85 \%$ to the increase in the value-added output of banks. Interestingly, the contribution of technology input becomes stronger after the financial crisis, consistent

\footnotetext{
${ }^{8}$ Unless otherwise specified, in the rest of the paper, "commercial banks", "U.S. banks" or "banks" refer to U.S. listed commercial banks, whose two-digit SIC code is 60.
} 
with the notion that technology has played a more important role in bank production in recent years. Moreover, the median net marginal product for technology on banks based on the estimated parameter range from $\$ 0.41-\$ 0.81$. These results suggest that technology investment is highly productive to U.S. banks.

Next, dramatic growth in technology spending by banks is documented. The median technology expenses of banks increased 285\% (from $\$ 1.16$ million to $\$ 3.31$ million) over the period, while the median number of employees and staff expenses increased by $70 \%$ and $100 \%$, respectively. Moreover, when the employment of a bank is measured as the number of employees it employs and the staff expense it pays, bank employment is positively and significantly correlated with their lagged technology spending in the crosssection. These results suggest that when banks spend more on technology, on average, their number of employees also increase. Furthermore, since the primary function of banks is to accept deposits from the public and to provide loans and advances of various forms, total loans and deposits, value-added, and number of branches are used as proxies to measure tasks of banks. Bank tasks are positively and significantly associated with their previousyear technology spending, suggesting that banks investing more in technology tend to create more tasks. The latter result is consistent with the task-based framework of Acemoglu and Restrepo (2018), which argue that exogenous technology adoption can create new tasks in the process of production for the next period.

This paper contributes to the literature in the following ways. First, it uses direct information on technology spending to investigate the impacts of technology on banks at 
the firm level, which overcomes the data limitation issue in the previous literature. ${ }^{9}$ The study is based on more than seven thousand annual observations of technology and communication expense of U.S. banks from S\&P Global Market Intelligence, which takes a "deep dive" into the banking sector and collects memo items, regulatory filings, supplemental financial schedules and financial reports from banks. The technology spending data in the analysis is mainly consisted of expenses paid for communications such as telephone and fax usage charges, internet data plans, and mobile phone and internet plans, data processing, and technology such as computers, wire services, modems, routers and switches, as well as software purchases and subscriptions to cloudbased services. This unique data set allows us to provide direct empirical evidence on the benefit of technology capital in bank production and the effect of technology spending on bank employment in details and fill in the gap of literature.

This paper estimates a value-added production function of banks to examine the contribution of technology capital to productivity, correcting for endogenous input choices and the measurement errors in technology capital. Comparing with the technology capital or IT capital in prior literature, this research is likely to have a more rigorous definition of technology capital as it is constructed from the annual technology and communication expense of banks assuming a perpetual inventory model with a depreciation ratio of $35 \%$ as well as a four-year linear depreciation schedule.

\footnotetext{
${ }^{9}$ Previous literature often uses survey data on technology investment, instead of the direct firm-level technology spending data.
} 
Finally, this paper is one of the first studies examining the extent to which the employment and tasks of U.S. bank are correlated with their technology investment. This empirical evidence focusing on the banking sector may also provide insights on the impacts of technology investment to other industries, as U.S. banks are intensive users of technology. Even though non-traditional financial intermediaries are growing in importance, banks are still preeminent in the financial system. ${ }^{10}$ Banks help individuals and firms fulfill transactions, issue deposits and make loans.

\subsection{Related Literature}

Regarding technology and employment relationship, some studies argue that technology and innovation are major drivers of employment growth of firms (e.g., Mokyr, 1992; Van Reenen, 1997; Bogliacino and Pianta, 2010; Harrison et al., 2014). Theoretically, technology investment has labor-saving effects (i.e., displacement effects), which impede employment, as well as employment-stimulating effects (i.e., compensation effects), which enhance employment. For example, there are a lot of cases in manufacturing and agriculture industries that machines replace the labor-intensive tasks (e.g., Bresnahan, 1999; Manyika et.at., 2013; Frey and Osborne, 2017; Bessen, 2017). On the contrary, the development of computers, as well as software, generates a huge demand for technician and services positions. In a task-based framework, Acemoglu and Restrepo (2018) argue

\footnotetext{
10 See Federal Reserve Bank of San Francisco (2001). What is the economic function of a bank? https://www.frbsf.org/education/publications/doctor-econ/2001/july/bank-economic-function/
} 
that technological automation tends to reduce employment, while the creation of new tasks by technology adoption increase employment since technology adoption will increase productivity, generate new tasks, deepen automation, and encourage capital automation.

There is also a clear example of the effect of technology adoptions on employment in the banking industry. Examining the effects of the introduction of automated teller machines (ATMs) on the employment of bank tellers, Bessen (2015) and David (2015) document that ATMs does not eliminate the teller job but increase it. He suggests that there more demand for teller since ATMs reduced the operating costs of banks and encouraged bank branching activities. However, early banking literature shows that the decline in the number of branches is delayed due to technology adoption (Saloner and Shepard, 1995). With a static production function framework assuming constant elasticity of substitution, Martin-Oliver and Salas-Fumas (2008) conclude that an additional 1 million Euros investment in IT may be substituted for 25 employees in Spanish commercial banks.

Regarding the effects of technology investment on productivity, most empirical studies found in economics, finance, and management literature largely employed survey data from large manufacturing firms or hospitals (e.g., Hitt and Brynjolfsson, 1996; Lee, McCullough, and Town, 2013). ${ }^{11}$ The results are mixed and inconclusive. For example, Baily (1986), Morrison (1997), Loveman (1994), and Berndt and Morrison (1995) find a negative or inconclusive relationship between use of technology and firm productivity,

\footnotetext{
${ }^{11}$ For instance, besides proprietary data, International Data Group (IDG) annual survey and Information Week annual survey are commonly used.
} 
while Lichtenberg (1995), Brynjolfsson and Hitt (1995, 1996, 2003), Bresnahan, Brynjolfsson, and Hitt (2002), and Bloom, Sadun, and Van Reenen (2012) show a positive correlation. ${ }^{12}$ Moreover, Hall and Khan (2003) show that the choice of technology adoption is between adopting it now or deferring the decision until later, but not a choice between adopting and not adopting, since that firms should invest or adopt new technologies at some points of time to stay competitive and provide better customer services.

Despite the intensity and importance of technology investment in the banking industry, research about its impact on U.S. banks is limited. One main reason is the lack of data on technology investment or spending, as this information is not typically disclosed to the public through bank financial reports and regulatory filings. Following a seminal work by Sealey and Lindley (1977), a few studies examine the impacts of technology investment using either survey data from U.S. banks or data from European banks. Based on data from 1984 to 2001, Berger (2003) find that technology significantly improves the quality of banking services and technological progress facilitates banking consolidation. With data from U.S. community banks from 1999-2001, DeYoung, Lang, and Nolle (2007) find that internet adoption improves performance for community banks, mainly through increased revenues from deposit service charges. ${ }^{13}$ Using a sample of 737 European banks from 1995 to 2000, Beccalli (2007) finds no relationship between total IT investment and

\footnotetext{
${ }^{12}$ See Brynjolfsson and Yang (1996) and Sichel and Oliner (2002) for reviews.

${ }^{13}$ Based on various proxies for technology, other studies also examine the role of technology in banking, including small business lending (e.g., Petersen and Ranjian, 2002), Internet usage (e.g., Hernando and Nieto, 2007; Hernández-Murillo, Llobet, and Fuentes, 2010; Dandapani, Lawrence, and Rodriguez, 2016). And, some earlier studies also include Hunter and Timme (1986) and Hamid and Verma (1994), which are based on banking data more than two decades ago.
} 
bank performance or efficiency. Examining the theory and measurement of financial intermediation, Philippon (2015) shows that the adoption of financial technology does not reduce intermediation costs.

In the aspect of technology on bank productivity, based on a survey data set on U.S. retail banking institutions from 1993-1995, Prasad and Harket (1997) show that increase in IT investment does not benefit banks in both productivity and performance. They argue that the use of IT is more of a strategic necessity for banks to stay in the competition. Martin-Oliver and Salas-Fumas (2008) examine the impact of information technology (IT) in the output of Spanish banks in the 1983-2003 period and find that one-third of output growth of banks can be explained by the growth in the stock of IT capital. Later, MartinOliver, Ruano, and Salas-Fumas (2013) provide similar results via different methodologies, using data from Spanish commercial banks during the 1992-2007 period.

To sum up, most of the studies related to technology in the banking literature are based on some proxies for technology usage (e.g., the number of ATMs, the transaction website adoption), limited survey data from U.S. bank, or data from European banks. Most of the papers use short-term data before the "network" era of computing, and thus unable to capture the full effects of the dramatic increase in technology adoption by banks. The results based on European bank data may not apply to the U.S. banks since there are significant differences between U.S. banking system and European banking system (e.g., capital market dependence, market structure, bank regulations, economic and banking 
industry size ${ }^{14}$ ). Lastly, most of the prior research focuses on the effects on bank productivity and the relationship between technology investment and bank performance. Little research has been done on how technology investment influences employments in commercial banks.

\subsection{Research Methodologies}

\subsubsection{Technology and Production}

Early literature does not properly differentiate among technology capital, nontechnology capital, and labor in their bank production models. For example, Martín-Oliver and Salas-Fumás (2008) and Martín-Oliver, Ruano, and Salas-Fumás (2013) first discuss and estimate the contribution of investment in information technology (IT) to the output of banks. In their specification, the bank-level output of the production function is total loan and deposit, and the bank-level inputs are IT capital and labor, which is defined as the number of employees (MRS Model, hereafter). The results from the MRS Model shows that one-third of output growth of banks can be explained by the growth in the stock of IT capital on their pre-crisis Spanish bank data. The issue with the MRS Model is that it does not take account of Non-IT physical capital, which includes but not limited to all machinery, equipment, and buildings, etc.

\footnotetext{
${ }^{14}$ GDP of Spain in 2015 is roughly $6.6 \%$ of the GDP of U.S. The total asset of Spanish banks in 2016 is about 2.7 trillion Euros based on a BBVA research report and the total asset of U.S. commercial banks at the same year is 12 trillion Dollars based on FRED economic data.
} 
Another issue when examining the impact of technology investment on banks is how to model the contribution of the increase of technology capital on revenue growth. Since there are well-known endogeneity issues on the estimation of production function (e.g., Marschak and Andrews, 1944; Ackerberg, Caves, and Frazer, 2006, 2015), as inputs are unobserved by the econometrician but may be observed by firm managers, standard approaches of parameter estimation will be biased due to simultaneity and correlation between inputs and productivity shocks.

Built upon the MRS Model, an augmented model is proposed. The production function in each bank is assumed to be a Cobb-Douglas, whose output is value-added $\left(Y_{i, t}\right)$ and inputs are technology capital $\left(T K_{i, t}\right)$, conventional capital or non-technology capital $\left(C K_{i, t}\right)$, and labor $\left(L_{i, t}\right)^{15}$. The analysis starts with the following log-transformation valueadded production function:

$$
y_{i, t}=\beta_{t k} t k_{i, t}+\beta_{c k} c k_{i, t}+\beta_{l} l_{i, t}+v_{i, t}
$$

where $y_{i, t}, t k_{i, t}, c k_{i, t}$ and $l_{i, t}$ are the natural logarithm of value-added, technology capital, conventional capital and labor of bank $i$ at year $t$, respectively. The primary interest is the $\beta_{t k} \mathrm{~s}$, which measure the technology capital contribution. The term $v_{i, t}$ represents the information that bank managers possess, which may be used for input selection. The major concern on the estimation of the above firm-level production function econometric to

\footnotetext{
${ }^{15}$ Several studies on IT-based production model have used a similar model but dividing the labor into IT labor and conventional labor (e.g., Loveman, 1994; Lichtenberg, 1995; Brynjolfsson and Hitt, 1996; Prasad and Harker, 1997; Lee, McCullough, and Town, 2013). Due to data availability, this analysis it not able to divide the labor information into IT labor and non-IT labor as theirs.
} 
correct for the endogenous bias in the estimation of the elasticity of the output with respect to technology capital, conventional capital and labor caused by the fact that the quantity of those inputs used in production might themselves be determined the value of the productivity shock (Griliches and Mareisse, 1998).

First, the production parameters using Olley and Pakes (1996) methodology (OP for short) is estimated. The OP model employs a two-step estimation on the parameters using a proxy variable to control the productivity shocks. It the OP methodology, the term $v_{i, t}$ can be decomposed as:

$$
v_{i, t}=\eta_{i, t}+\epsilon_{i, t}
$$

where $\epsilon_{i, t}$ is a normally distributed idiosyncratic error term. $\eta_{i, t}$ is assumed to be the unobserved productivity or technical efficiency term and evolves according to a first-order Markov process, $\eta_{i, t}=E_{t}\left(\eta_{i, t} \mid \eta_{i, t-1}\right)+\zeta_{i, t}=g\left(\eta_{i, t-1}\right)+\zeta_{i, t}$, where $\zeta_{i, t}$ is a random shock component, which is assumed to be uncorrected with the productivity term, $c k_{i, t}, l_{i, t}$ and $t k_{i, t-1}, c k_{i, t-1}, l_{i, t-1}$.

There are several key assumptions in the OP methodology. First, it assumes that $\eta_{i, t}$ is observed by the firm manager and that $\eta_{i, t}$ is used by the firm manager to decide the amount of inputs. Second, it assumes that firm-level investments $\left(i n v_{i, t}\right)$ is a function of $c k_{i, t}, l_{i, t}$ and $\eta_{i, t}$, that $i n v_{i, t}$ is strictly monotone in $\eta_{i, t} \cdot \eta_{i, t}$ is scalar unobservable in $i_{i, t}=$ $i($.$) . Third, the levels of i n v_{i, t}, c k_{i, t}$ and $l_{i, t}$ have been chosen prior to period $t$. The level of $t k_{i, t}$ is then decided after the realization of the shock $\zeta_{i, t}$. In other words, the 
productivity shock proxy must be monotonically increasing with respect to the true productivity shock. These assumptions ensure the invertibility of $i n v_{i, t}$ in $\eta_{i, t}$, and lead to the following partially-identified model:

$$
\begin{aligned}
y_{i, t}=\beta_{t k} t k_{i, t} & +\beta_{c k} c k_{i, t}+\beta_{l} l_{i, t}+h\left(i n v_{i, t}+c k_{i, t}+t k_{i, t}\right)+\epsilon_{i, t} \\
& =\beta_{l} l_{i, t}+\psi\left(i n v_{i, t}, c k_{i, t}, t k_{i, t}\right)+\epsilon_{i, t}
\end{aligned}
$$

where $\psi$ is approximated with a second order polynomial series in technology capital and conventional capital. Equation (3-3) can be estimated by non-parametric approach. In the first stage, the production function parameters are estimated by taking advantage of the Markovian nature of the productivity process and the assumptions above as moment conditions. In the second stage, the residual term is derived as follows:

$$
y_{i, t}-\widehat{\beta}_{l} l_{i, t}=\beta_{t k} t k_{i, t}+\beta_{c k} c k_{i, t}+g\left(\eta_{i, t-1}+\vartheta_{i, t}\right)+\epsilon_{i, t}
$$

where $\mathrm{g}($.$) is typically unspecified and approximated by an n$-th order polynomial and $\vartheta_{i, t}$ is an indicator function for the attrition in the market.

Besides the OP methodology, the production parameters using dynamic panel data (DPD) model of Arellano and Bond (1991), Arellano and Bover (1995), and Blundell and Bond $(1998,2000)$, firm fixed-effects (FE) approach and traditional ordinary least squares 
(OLS) approach are also estimated. ${ }^{16}$ In these models, the last term $v_{i, t}$ in Equation (3-1) can also be further decomposed into four components:

$$
v_{i, t}=a_{i}+\gamma_{t}+\eta_{i, t}+\epsilon_{i, t}
$$

where $a_{i}$ is a time-invariant firm fixed-effect and $\gamma_{t}$ is a time-varying productivity shock. These factors are likely to be related to the observed inputs. $\eta_{i, t}$ is an unobserved productivity term, which might be correlated with the observed inputs, and evolves as an autoregressive process, $\eta_{i, t}=\rho \eta_{i, t-1}+\omega_{i, t}$, where $\omega_{i, t}$ is a pure stochastic component. The innovation on unobserved productivity, $\omega_{i, t}$, is assumed to be uncorrelated with the observed inputs. The last term, $\epsilon_{i, t}$, reflects a productivity shock, which might be correlated with the observed inputs and might evolve as a moving average process. The impact in time for $\epsilon_{i, t}$ might also last for a long period.

Even if the term $v_{i, t}$ consists of a firm fixed-effect and a component of the evolving productivity, it is likely to be correlated with the observed inputs. By solving for $\eta_{i, t-1}$ and substituting it into the empirical model in Equation (3-1), a dynamic form is generated as follows:

\footnotetext{
${ }^{16}$ Levinsohn and Petrin (2003) aimed to overcome the empirical issue that there are usually quite a lot of zeros in the investment data and proposed to use intermediate inputs (materials) to estimate the production shock. However, it is too difficult to define what are the intermediate inputs (materials) of banks. See MartínOliver, Ruano and Salas-Fumás (2013) for some attempts using this methodology.
} 


$$
\begin{aligned}
y_{i, t}=\rho y_{i, t-1} & +\beta_{t k} t k_{i, t}-\rho \beta_{t k} t k_{i, t-1}+\beta_{c k} c k_{i, t}-\rho \beta_{c k} c k_{i, t-1} \\
& +\beta_{l} l_{i, t}-\rho \beta_{l} l_{i, t-1}+a_{i}-\rho a_{i}+\gamma_{t}-\rho \gamma_{t-1}+\omega_{i, t} \\
& +\epsilon_{i, t}
\end{aligned}
$$

An equation can be obtained by renaming the respective coefficients and grouping the error components, as Equation (3-7):

$$
\begin{aligned}
y_{i, t}=\delta_{1} y_{i, t-1} & +\delta_{2} t k_{i, t}+\delta_{3} t k_{i, t-1}+\delta_{4} c k_{i, t}+\delta_{5} c k_{i, t-1}+\delta_{6} l_{i, t} \\
& +\delta_{7} l_{i, t-1}+a_{i}{ }^{*}+\gamma_{t}{ }^{*}+\omega_{i, t}{ }^{*}
\end{aligned}
$$

where the common factor restrictions are $\delta_{3}=-\delta_{1} * \delta_{2}, \delta_{5}=-\delta_{1} * \delta_{4}$ and $\delta_{7}=-\delta_{1} *$ $\delta_{6}$, with $a_{i}{ }^{*}=a_{i}(1-\rho), \gamma_{t}{ }^{*}=\gamma_{t}(1-\rho)$ and $\omega_{i, t}{ }^{*}=\omega_{i, t}+\epsilon_{i, t}$. Assuming all the common factor restrictions hold, the traditional ordinary least squares (OLS) approach will yield consistent parameters only when $E\left(a_{i}{ }^{*} x_{i, t}\right)=0, E\left(\omega_{i, t} x_{i, t}\right)=0$ and $E\left(\epsilon_{i, t} x_{i, t}\right)=0$, where $x$ are state variables in the production estimation. Consistent parameters can be obtained in firm fixed-effect model only if $E\left(\omega_{i, t} x_{i, t}\right)=0$ and $E\left(\epsilon_{i, t} x_{i, t}\right)=0$.

The DPD approach provides consistent parameters under less restrictive assumptions than the OLS approach and the fixed-effects (FE) approach. A system GMM approach that simultaneously estimates the production function using both levels and difference specifications is adopted. The system GMM estimators are designed for dynamic "small-T, large-N" panels that may contain fixed-effects and idiosyncratic errors that are heteroskedastic and correlated within, but not across firms. 
Managers are likely to choose their input levels because productivity is known to them (Marschak and Andrews, 1944). Since measurement errors and endogeneity may exist in the production input measures, the OLS estimators will be biased towards zero. The DPD approach allows for a time-invariant firm fixed-effect, which it is important since there are different business strategies and production inputs among banks in different locations and various customer focus. Nonetheless, there is no agreement in academics about which parameter estimation approach is more appropriate. In this paper, all four models - OP, DPD, FE, and OLS are adopted, to evaluate the sensitivity of the estimated parameters under different kinds of identification assumptions.

\subsubsection{Technology and Employment}

To some extent, bank technology investment should be exogenous [see the Static Model of Acemoglu and Restrepo (2018)], even if there exists some endogeneity concern that bank employment may also drive its technology investment. The technology adoption of banks, to a large extent, is driven by the general economic conditions, the competitive environment and the rapid development of technology. ${ }^{17}$ Banks should confront the difficulty and challenge of the rapid development and creation of new technologies: online banking, data security, DLT system, clouds, etc. Vítor Constâncio, Vice-President of the European Central Bank, states that "beyond increased competition from non-banks, the

\footnotetext{
${ }^{17}$ See an article from The Telegraph on April 2, 2017, "Mark Carney warns of fintech threat to traditional banks".
} 
banking sector faces competition from Financial Technology (FinTech) firms..."18 The indeterminacy nature of the banking system and the astonishing adoption of technology, not the supply of ordinary employees, have made radical transformations in the way how banks do business and continue to change even further.

Bank employment is highly persistent (continuous workflow and difficult to hire or fire) over time and that there exist automation of old tasks and creation of new tasks [see Figure 2 in Acemoglu and Restrepo (2018)]. There exist labor-saving effects as well as employment-stimulating effects in technology investment. Hence, one would also like to see whether banks with a higher level of technology adoption would have more employees in the next period.

It is extremely important to control heterogeneity in size in the analysis, considering one need to compare technology spending among firms with equal firm size and examine the effect of technology spending on firm-level employment. The technology expense of banks regresses on their size in the cross-section. Specifically, the following specification using ordinary least squares (OLS) is estimated. The residual technology spending obtained from this regression is the independent variable of interest.

$$
\text { LnTechSpending }_{i}=\beta_{0}+\beta_{1} \text { Firm Size }_{i}+\varepsilon_{i}
$$

\footnotetext{
${ }^{18}$ See a lecture on July 7, 2016, titled "Challenges for the European banking industry," by Vítor Constâncio at the conference of "European banking industry: What's next," organized by the University of Navarra.
} 
Since it is also needed to compare the differentials of employment and tasks of firms at the equal size, residual employment and tasks are also obtained by replacing the dependent variable of equation (3-8) to the measures of employment and tasks of banks.

With residual firm-level technology spending and employment estimated, one can examine the relationship between employment and technology spending in the crosssection by the following equation using OLS model with standard errors are clustered at the firm-level and are heteroscedasticity-robust:

$$
\begin{aligned}
& \text { ResEmployment }_{i, t} \\
& =\beta_{0}+\beta_{1} \text { ResTechSpending }{ }_{i, t-1}+\beta_{2} M T B_{i, t-1} \\
& +\beta_{3} \text { Leverage }_{i, t-1}+\beta_{4} R O A_{i, t-1} \\
& +\beta_{5} \text { NonIntIncome }_{i, t-1} \\
& +\beta_{6} \text { Tier1Capital Ratio }_{i, t-1}+\eta_{i}+\alpha_{t}+\varepsilon_{i, t}
\end{aligned}
$$

where $\eta_{i}$ and $\alpha_{t}$ represent firm and year fixed effects, respectively. The coefficient of interest, $\beta_{1}$, measures the cross-sectional relationship between the previous-year technology spending and employment at the firm-level. The effect is pooled across crosssections in the panel, net of interacted firm characteristics within each year.

To examine how a firm's technology investment influences its tasks, a similar model is run as what in examining the relationship between employment and technology spending as in Equation (3-9), by replacing residual employment into residual tasks in the right-hand side of the equation, as follows: 


$$
\begin{aligned}
& \text { Residual Tasks }{ }_{i, t} \\
& =\beta_{0}+\beta_{1} \text { ResTechSpending }{ }_{i, t-1}+\beta_{2} M T B_{i, t-1} \\
& +\beta_{3} \text { Leverage }_{i, t-1}+\beta_{4} R O A_{i, t-1} \\
& +\beta_{5} \text { NonIntIncome } e_{i, t-1} \\
& +\beta_{6} \text { Tier1Capital Ratio }_{i, t-1}+\eta_{i}+\alpha_{t}+\varepsilon_{i, t}
\end{aligned}
$$

\subsection{Data Description}

\subsubsection{Data Source}

The empirical analysis uses annual data on firm characteristics of U.S. listed commercial banks (two-digit SIC code: 60) from Compustat banking database and S\&P Global Market Intelligence’s (formally SNL Financial) banking database from 2000- 2017. The technology and communication expense, the total number of offices, and the number of automatic teller machines (ATMs) are collected from the S\&P Global Market Intelligence, while all other annual financial characteristics are collected from Compustat. ${ }^{19}$

The technology and communication expense (technology expense) reported in the S\&P Global Market Intelligence database is primarily constructed based on U.S. GAAP standard FAS No. 86. The item includes expenses paid for communications such as

\footnotetext{
${ }^{19}$ Missing financial characteristics in year $t$ are replaced by estimates from this formula: $\operatorname{Var}_{i, t}^{x}=$ $\left(\operatorname{Var}_{i, t+1}^{x}+\operatorname{Var}_{i, t-1}^{x}\right) / 2$, where $\operatorname{Var}_{i, t}^{x}$ is the information of $x$ of bank $i$ in year $t$.
} 
telephone and fax usage charges, internet data plans, and mobile phone and internet plans, data processing and technology such as computers, wire services, modems, routers and switches, as well as software purchases and subscriptions to cloud-based services. The value of technology and communication expense is constructed via original data from the bank's financial reports and bank regulatory filings. For instance, the 2015 technology expense of Citigroup (Ticker: C) in the sample is $\$ 6,581$ million, which comes from the technology/communication item (\$6,581 million) in its annual report (10-K). The 2015 technology expense of Bank of America (Ticker: BAC) is $\$ 3,938$ million, which comes from the telecommunications item ( $\$ 823$ million) and data processing item $(\$ 3,115$ million) in its annual report. The 2015 technology expense of Community First Bancorp, Inc (Ticker: CMFP) is $\$ 399,000$, which comes from data processing item $(\$ 162,616)$, telephone item $(\$ 59,150)$, internet banking item $(\$ 87,643)$ and ATM expenses item $(\$ 89,771)$ on its annual report. The 2015 technology expense of Pandora Bancshares, Inc. (Ticker: PDRB) is $\$ 736,000$, which comes directly from tech \& communications expense item $(\$ 736,000)$ on its bank regulatory filings, even if a data processing item $(\$ 505,000)$, which is smaller than the reported number in its bank regulatory filings, is reported on its annual report.

\subsubsection{Variable Construction}

Value added is commonly used as a measure of production output. It represents the wealth created through a firm's production process. Nonetheless, there is no agreement in academics and practitioners about how to define and measure the output of the service 
industries, especially for banks (see Griliches, 1992; Griliches, 1994; Triplett and Bosworth, 2004; Prasad and Harker, 2007; Berger and Humphrey, 2008; Basu, Inklaar, and Wang, 2011).

According to the Bureau of Economic Analysis (BEA), "banks are compensated for some services by a portion of the interest that they charge on loans or by a reduction in the interest rates that they pay to depositors - rather than by charging explicit fees." (Hood, 2013). The value-added output measure for banks is operationalized as its net interest income, which measures the difference between the revenue generated from a bank's assets and the costs of its materials and services (liabilities). The labor input is measured by the compensation and benefits of employees (staff expense) as it can capture the difference in the skill level of employees (as in, for example, Prasad and Harker, 1997; Brynjolfsson and Hitt, 2003; Levine and Warusawitharana, 2014). Conventional capital is defined as total assets excluding intangible assets and technology capital. The technology investment in the Olley and Pakes (1996) methodology is measured as investment expenditure.

The complication for the construction of technology capital is that S\&P Global Market Intelligence and Compustat do not report the actual value of the banks' technology capital stock. Hence, the value of technology capital is constructed using the technology and communication expense recorded in S\&P Global Market Intelligence each year. First, the paper follows Martín-Oliver, Ruano, and Salas-Fumás (2013) and estimates the physical technology capital stock from the annual technology expense of banks assuming a perpetual inventory model with a depreciation ratio of $35 \%$. Alternatively, a four-year linear depreciation schedule to construct the annual physical technology capital stock for 
each bank are also used, as in Lee, McCullough, and Town (2013). In the Financial Accounting Manual for Federal Reserve Banks, the maximum estimated useful life for standard technology personal computers (PCs) is three years, and state-of-the-art technology PCs is four years, while it is six years for operating equipment with $10 \%$ salvage value. ${ }^{20}$

It should be recognized that technology expense likely includes both physical technology equipment and services. There is no practical way for us to disentangle the components of the expense. To address the concern whether it is a proper technology capital measure, its relation to the adoption of ATMs is examined. Regression of the natural $\log$ of technology capital estimated from perpetual inventory model and linear depreciation schedule, respectively, to the natural log of the number of ATMs with standard errors that are clustered at the bank-level and are heteroscedasticity-robust is adopted. The estimated parameter of the number of ATMs is positive (0.892 and 0.887$)$ and highly statistically significant ( $t$-statistic: 35.33 and 35.18). In this univariate regression, the number of ATMs accounts for a very large portion of the overall variation in technology capital $(R$-squared: 0.725 and 0.728$)$. The correlation between the two measures of technology capital and the number of ATMs are 0.852 and 0.854 , and statistically significant at the $1 \%$ level. This evidence gives us confidence in the validity of the technology capital measure.

\footnotetext{
${ }^{20}$ For more details, please see Section 30.78 Maximum Useful Lives and Salvage Values Table of the document. https://www.federalreserve.gov/federal-reserve-banks/fam/chapter-3-property-andequipment.htm
} 
The technology capital measure is, to some extent, different from the technology capital or IT capital in previous bank production literature. In the extent of U.S. banks, Prasad and Harker (1997) use survey data from large retail banks on their IT spending during 1993-1995 to construct the IT-related expense. In the extent of Spanish banks, Martín-Oliver and Salas-Fumás (2008) and Martín-Oliver, Ruano, and Salas-Fumás (2013) construct their total IT capital of banks as the sum of the book value of IT capital on the asset side of the balance sheet and the estimated IT capital stock. The huge differential of inputs in the production functions in this paper and the previous papers might lead to the difference of the results comparing to theirs. The ratio of IT capital and non-IT capital in Prasad and Harker is about 0.106, while the ratio of IT capital and physical capital in the year 1983 is 0.105 and that in the year 2003 reaches 0.621 in Martín-Oliver, Ruano, and Salas-Fumás (2013), according to the summary statistic tables of their papers. The ratio of technology capital and conventional capital in this paper is, on average, only $0.39 \%$ when technology capital is estimated using perpetual inventory model and $0.38 \%$ when technology capital is estimated using linear depreciation schedule. The ratio of the mean (median) of the technology expense related to total current operating expense is $4.12 \%$ (3.63\%), which is close to the number in Mai, Speyer, and Hoffmann (2012) and the McKinsey report. Comparing with previous studies, this analysis is likely to have a much more rigorous definition of technology capital and a broader sense of conventional capital as it includes tangible capital that is not technology capital.

As the paper constructs the technology capital measure using expense information and assumption of depreciation, there are possibilities that it systematically over- or under- 
represent the true value of technology capital of each bank. The over- or under- estimations is a common issue on production analysis, which generally relies on survey or accounting information that naturally embodies assumptions and depreciation and expenditure classification. The more concern issue is that how one can estimate consistent parameters, given there exist measurement errors in the input and endogenous issue between input and productivity shocks as in the discussion in the previous sections.

The employment of a bank is measured as the number of employees it employs and the staff expense it pays. Although the number of employees and staff expense gives us an idea of how employees a bank has, it suffers from one major drawback: it does not adjust for the bank's size, thus making it hard to compare how many staff one bank employs related to another. Similarly, although technology expense gives us an idea of how much technology investment a bank is doing, it is very difficult to compare how much one bank is investing relative to another. Hence, residual employment and technology expense, which can be used to compare employment and technology expense among firms with equal firm size, is used as key variables in the regressions.

The bank task measures are extremely difficult to quantify, not to say to distinguish these tasks into old tasks and new tasks recently generated. The traditional banking business is to accept deposits and make loans. ${ }^{21}$ Since the primary functions of banks is (a) to receive various types of deposits from individuals, businesses, financial institutions, and governments, and (b) to lend money in various forms to businesses, other financial

\footnotetext{
${ }^{21}$ See Bhattacharya and Thakor (1993) for a review.
} 
institutions, individuals, and governments, ${ }^{22}$ the total loans and deposits of a bank should be a proper proxy of its tasks. As the main business of banks is to collect deposits and make loans, the total loans and deposits of a bank should represent the amounts of tasks it has. Alternatively, value added and the number of branches are also employed as two proxies of bank tasks. The value-added reflects the wealth created by a bank through the production process. The number of branches of a bank reflects its complexity. Hence, both can be used as a measure of the amounts of tasks.

Other variables used in this study include are as follows. Bank's size (Firm Size) is defined as the natural log of market capitalization at the end of the fiscal year. Market to book ratio (Market to Book) is defined as the ratio of total book assets to total book equity. Leverage ratio (Leverage) is defined as the ratio of total book assets to total book equity. Return on Assets is defined as the ratio of earnings before extraordinary plus depreciation and amortization to total book assets. Non-interest income ratio (Non-Interest Income) is defined as the ratio of banks' non-interest income to the sum of net interest income and non-interest income. Risk-adjusted tier1 capital ratio (Tier 1 Capital Ratio) is obtained from Compustat.

\footnotetext{
${ }^{22}$ Diamond and Dybvig (1986) argue that main functions of bank as asset services to the borrowers, liability services to the depositors, and transformation services.
} 


\subsubsection{Summary Statistics}

The definitions for all variables used are listed in Table A1 in the appendix. Reducing noises in the analysis, firms with fewer than five consecutive years of technology expense and total asset information during the sample period are excluded. Firms with missing values of the relevant variables are also excluded. Finally, all the variables are winsorized at the $1 \%$ and $99 \%$ tails of the distributions to avoid the influence of extreme observations. The final sample consists of 8,030 firm-year observations for 781 banks during the 2000-2017 period.

Figure 3-1 displays bank technology expense trends over the sample period. In 2017 dollars, the median of bank technology spending jumps to $\$ 3.31$ million in 2017 from $\$ 1.16$ million in 2000. This figure shows that there exists a steady increase in the technology spending of banks for most of the years over this time. ${ }^{23}$

Table 3-1 reports the summary statistics of the regression variables used in this paper. The mean (median) market capitalization in the panel is $\$ 1,191$ million $(\$ 110.16$ million), while the mean (median) total assets is $\$ 7,613$ million (\$966 million) ]. The technology expense of $\$ 15.69$ million and a median of $\$ 1.58$ million. In term of production variables, the mean (median) of value-added, technology capital estimated with a perpetual inventory model, its corresponding conventional capital, labor, and investment is $\$ 204.96$

\footnotetext{
${ }^{23}$ When one keeps firms that record technology and communication expense in each year during 2000-2017 and illustrate their trends (medians) of technology and communication expense, a monotonic increase in the technology spending in the sample period is also found. There is a total of 97 firms in this sample. See Figure B1 of the appendix for details.
} 
(\$31.19) million, \$35.06 (\$3.39) million, \$7,329.40 (\$954.60) million, \$113.31 (\$14.55) million, and \$8.62 (\$1.56) million, respectively. The typical bank has an average (median) total loans and deposits of $\$ 9,225.38$ (\$1,398.39) million and average (median) number of branches of 57.766 (15.00). On average (median), it employs 1,648 (264) employees and pays $\$ 113.31$ (\$14.55) million as staff expense.

Figure 3-2 illustrates the evolution of the median of technology spending, the number of employees and staff expense of banks during the sample period. All monetary values are adjusted for inflation using GDP deflator and are normalized to equal one in the year 2000. The figure shows that technology expense grew about $250 \%$, much faster than the number of tasks and employment, from 2000 to 2017. In the meanwhile, the median number of total loans and deposits increased by about $100 \%$, and the median number of employees increased by about $70 \%$. The dramatical increase of expense on technology draws the attention to evaluate its contribution to the production of banks, and its relationship with employment. ${ }^{24}$

\footnotetext{
${ }^{24}$ When one keep firms that record technology and communication expense in each year during 2000-2017 and illustrate the evolution of the median of technology spending, the number of employees and staff expense of those banks, similar evolution patterns in the sample period are also found. There is a total of 97 firms in this sample. See Figure B2 of the appendix for details.
} 


\subsection{Empirical Results}

\subsubsection{Technology in Production}

As stated in the methodologies section, the analysis starts by estimating the contribution of technology capital on banks using a firm-level value-added production function and examine whether banks benefit from their technology investments. The production function estimates with the model that technology capital is calculated from a perpetual inventory model are presented in Table 3-2.

The first column represents the parameter estimated from the Olley and Pakes (OP, 1996) methodology. The estimated parameter for technology capital in the OP model is 0.085 and statistically significant at the $1 \%$ level. The variable that serves as a proxy for productivity shocks is investment expenditure, which is used to control for the simultaneity between the productivity shocks and the labor input decision. The $p$-value associated with the null hypotheses of constant return to scale $\left(\beta_{t k}+\beta_{c k}+\beta_{l}=1\right)$ is 0.285 . Standard errors for the OP model are generated via bootstrap based on 200 replications. The results indicate that technology capital is very productive.

Columns (2), (3) and (4) report the DPD, FE and OLS estimates. The parameter estimate for technology capital in the DPD model is 0.112 and statistically significant at the $1 \%$ level. It is greater than that from the OP model. Common factor restrictions are rejected, while the null hypotheses of constant return to scale are not. The parameter estimates for technology capital are 0.054 from FE model and 0.061 from OLS model. All 
are statistically significant at the $1 \%$ level. Common factor restrictions and constant return to scale are rejected for both models.

These results are also consistent with the literature on the production function parameter estimation and the notion that the production input choices could be endogenous. The results also confirm the worry on the measurement errors on the technology capital input. The measure is likely to underestimate the true value of technology capital. Besides, the investment proxy in the OP (1996) methodology is much easier to observe and more precious, as these numbers are usually disclosure in their financial reports.

The paper further examines the implications of the production function parameter estimates on the historical contributions of the technology capital input of banks to their value-added. To measure the historical contribution of technology capital, the difference in each bank's value-added under 2017 and 2003 technology capital input levels are calculated. Value-added grew an average of $225 \%$ over this period - an approximately $5.78 \%$ compound growth rate. Technology capital grew an average of 554\% over this period - an approximately $9.70 \%$ compound growth rate. On average, technology inputs accounted for an approximately $12.85 \%$ increase in value-added output of banks. The result suggests that there exists a huge economically significant return from technology investments of banks during this period.

Next, whether the contribution of technology investment is greater than its cost is assessed. The median net marginal product for technology on banks based on the estimated parameter range from $\$ 0.41$ for the FE model ( $p$-value $<0.001)$ and $\$ 0.81$ for the DPD 
model $(p$-value $<0.001) .{ }^{25}$ Even if technology capital is assumed to have an average service life as little as three years, ${ }^{26}$ the median net marginal product still ranges from $\$ 0.22$ for the FE model and $\$ 0.61$ for the DPD model and be greater than zero at statistical significance at the $1 \%$ level. Their results suggest that the substantial increases in technology investment would be beneficial.

These net marginal products are similar to the estimations in other industries. For instance, the net marginal product is $\$ 0.67$ for technology in Brynjolfsson and Hitt (1996), whose sample is of U.S. large firms (Fortune 500). They range from $\$ 0.73$ to $\$ 1.29$ in Lee, McCullough, and Town (2013), whose focus is California hospitals.

Concerning there may be systematically over- or under- represent the true value of technology capital of each firm, alternatively, the production function parameters using technology capital stock estimated from a four-year linear depreciation schedule as a production input are estimated. Table 3-3 reports the result. The estimated parameters for technology capital (0.079 from OP, 0.083 from DPD, 0.050 from FE and 0.053 from OLS) are quantitively similar with that in Table 3-2, where technology capital is estimated using a perpetual inventory model. Common factor restrictions are quite similar with those in Table 3-2. The null hypotheses of constant return to scale cannot be rejected in the OP

\footnotetext{
${ }^{25}$ Following Brynjolfsson and Hitt (1996), the gross marginal product for technology capital is the output elasticity, which is the estimated parameter to technology capital, multiplied by the ratio of output to technology capital input. Hence, the net marginal product is calculated as gross marginal products subtract $14 \%$.

${ }^{26}$ Thus, the net marginal product is calculated as gross marginal products subtract $33.33 \%$.
} 
model and the DPD model but rejected in the other models. The levels of statistical significance are consistent as well. The consistent results provide further evaluation of the sensitivity of the estimated parameters under different kind of construction methods on technology capital stock.

Based on the estimations in Table 3-3, technology input, on average, accounted for an approximately $9.27 \%$ increase in value-added output of banks. The median net marginal product for technology range from $\$ 0.32$ for the FE model and $\$ 0.58$ for the DPD model and are greater than zero at statistical significance at the $1 \%$ level. Assuming technology capital have an average three years' service life, the median net marginal product still range from $\$ 0.10$ to $\$ 0.38$ and are greater than zero at statistical significance at the $1 \%$ level.

\subsubsection{Technology and Employment}

This subsection explores whether their previous-year technology spending can explain bank employment. First, the residual technology spending of banks is computed using equation (3-8). Columns (1) to (4) of Panel A, Table 3-4 report the cross-sectional regression results of the natural log of technology expense on firm size, which is the log of market capitalization at the end of the fiscal year, for four years: 2000, 2005, 2010 and 2015. The estimated coefficients of firm size are highly consistent in each of the crosssection regression: 0.828 in 2000, 0.855 in $2005,0.658$ in 2010 , and 0.782 in 2015 . All the estimated coefficients are highly statistical significance at the $1 \%$ level ( $t$-statistics range from 22.35 to 32.26 ). The $R$-squared range from 0.655 to 0.818 . Columns (5) reports the 
results based on a pooled regression for all the cross-sections from 2000 to 2017. Again, the estimated coefficient is 0.767 , with $t$-statistics of 35.14 and $R$-squared of 0.760 . Thus, through this regression, residual technology spending is computed, and the effects of firm size on technology spending are excluded. Using the same method, residual employment and residual tasks are calculated adjusting the effects of firm size. These results are reported in Table A2 of the appendix.

Panel B of Table 3-4 shows the results on the persistence tests of residual technology spending and residual employment of banks. The residuals in year $t$ are strongly correlated with their corresponding residuals in year $t-1$. Specifically, in Column (1), the estimated coefficient for residual technology spending is 0.905 , with $t$-statistics being 133.59 and $R$-squared being 0.823 . The coefficients of the residual number of employees and residual staff expense are 0.901 and 0.884 , respectively, both being highly statistically significant, as in Column (2) and (3). These results indicate the residual technology spending and residual employment of banks are highly persistent over time.

Panel C of Table 3-4 presents the results on the correlations of residual technology spending and residual employment. The residual technology spending in year $t$ is strongly correlated with the residual technology spending in year $t$-1, with the correlation being 0.908. Similarly, the correlation of residual number of employees (staff expense) in year $t$ with residual number of employees (staff expense) in year $t-1$ is $0.900(0.880)$. These findings suggest that there exists a permanent firm effect on technology spending and firm employment. Moreover, residual employment is correlated with residual technology 
spending in the previous year. The correlation is 0.579 for residual number of employees and 0.599 for residual staff expense.

After documenting the persistence of technology spending and employment at the firm-level, the extent to which bank employment is related to the previous-year technology spending is examined. Table 3-5 reports the results from Equation (3-9). Overall, the results provide evidence that the firms that invest more in technology have higher employment, controlling for firm size, growth strategy, financing, performance, fee income ratio, and financial strength.

Columns (1) and (2) of Table 3-5 report the univariate regression results. When the dependent variable is residual number of employees, the estimated coefficient of the previous-year residual technology spending is positive (0.348) and statistically significant at the $1 \%$ level. Concerning residual staff expense, the estimated coefficient is also positive (0.390) and statistically significant at the $1 \%$ level. The baseline result suggests that banks with more technology spending employ more staff.

Columns (3) and (4) present the multivariate regression results. Positive relations between firm-level employment and technology spending are evident. The estimated coefficients of the previous-year technology spending variable are 0.196 when the dependent variable is residual number of employees and 0.202 when the dependent variable is residual staff expense. Both display statistically significant at $1 \%$ level. Aside from the coefficients of the main interests, bank employment is negatively correlated with the previous-year market-to-book ratio and return on assets and positively correlated with the 
previous-year leverage and non-interest income. The result is also consistent with Van Reenen (1997), which finds a positive and significant effect of innovations on employment based on the British firm-level panel data. ${ }^{27}$

The positive relationship between employment and technology spending holds consistently throughout the panel, which plots the relationship between residual number of employees (Figure 3-3) and residual staff expense (Figure 3-3) and residual technology spending for nine cross-sections, 2001, 2003, 2005, 2007, 2009, 20011, 2013, 2015 and 2017.

Next, the extent to which firm-level tasks are related to the previous-year technology spending is examined, using a similar approach as in the previous analysis. Table 3-6 reports the results from Equation (3-10). When the dependent variable is residual loans and deposits, the estimated coefficients of the previous-year technology spending variable are 0.308 in the univariate regression, as in Column (1), and 0.137 in the multivariate regression, as in Column (4), with statistical significance at the $1 \%$ level. When the dependent variable is residual value added, the estimated coefficients are 0.301 in the univariate regression and 0.161 in the multivariate regression, and statistically significant at the $1 \%$ level, as in Column (2) and (5). When the dependent variable is

\footnotetext{
${ }^{27}$ The analysis are rerun by measuring size using book asset values rather than the market value of equity, based on the idea that book asset values reflect both debt plus equity and thus may constitute a better proxy for the scale of the firm. Results are very similar. See Table A3 of the appendix for details. Moreover, the analysis are rerun by measuring firm size as total loans and deposits, based on the idea that the basic business model of banks is to make loans and collect deposits and most of their assets should be in loans and deposits and thus may constitute a better proxy for the scale of the firm. Similar results are reported, as in Table A4 of the appendix.
} 
residual number of branches, the estimated coefficients are 0.324 in the univariate regression and 0.191 in the multivariate regression, and statistically significant at the $1 \%$ level, as in Column (3) and (6). Bank tasks are positively correlated with the previous-year leverage and negatively correlated with the previous-year market-to-book ratio and return on assets, non-interest income, and tier 1 capital ratio.

Overall, the regression result shows that bank tasks are positively and significantly associated with their previous-year technology spending, supporting the notion that technology adoption creates new tasks (Acemoglu and Restrepo, 2018). The result also implies that technology help banks to expense their business.

Collectively, the cross-sectional results provide strong evidence that bank employment and tasks are positively related to their previous-year technology spending, implying that on average, banks adopting more technology tend to increase their number of employees and create more tasks.

\subsubsection{Robustness Checks}

Results for robustness checks are presented in this subsection. It also re-estimates the main analysis by 1) excluding too-big-to-fail (TBTF) banks, which are also called systematically important financial institutions (SIFIs), and 2) keeping observations only in the post financial crisis period (2010-2017). It is believed that the extremely large banks operate in very different models and are under different degrees of regulation, supervisions, 
and supports. To ensure those banks do not overly influence the results, the main analysis excluding banks whose gross total assets exceed $\$ 50$ billion in 2017 dollars are reestimated. In recent years, the banking industry gradually recovered from the financial crisis. Banks have adapted well to the new business environment and regulations. ${ }^{28}$ They have bolstered their balance sheets and adjusted product portfolios, business strategies, and even operation models. Thus, the bank production process or business model might be quite different during the pre- and post- financial crisis period. To ensure the results hold in the new era, the main analysis is re-estimated in the post-financial crisis period (20102017).

Panel A, Table 3-7 shows the production function parameter estimates. While toobig-to-fail banks excluded, as in Columns (1) to (4), The estimated parameters for technology capital (0.065 in OP, 0.114 in DPD, 0.064 in FE, and 0.070 in OLS) are largely consistent with those estimated to the full sample as in Table 3-2, suggesting technology capital also make meaningful and significant contributions to bank production. Columns (4) to (8) presents the results for the post financial crisis period. The estimated parameters for technology input are quantitatively and qualitatively greater than that in Table 2 . While they are 0.085 in OP, 0.112 in DPD, 0.065 in FE, and 0.067 in OLS during the full sample period, the estimated parameters are now 0.131 in OP, 0.239 in DPD, 0.133 in FE, and 0.107 in OLS in the post financial crisis period, indicating that technology investment played a greater role and was more beneficial, and that technology capital was highly

\footnotetext{
${ }^{28}$ See a report by Bank for International Settlements, Committee on the Global Financial System on January 2018, "Structural changes in banking after the crisis," CGFS Papers, No. 60.
} 
productive in recent years. The consistent results provide further evaluation of the sensitivity of the estimated parameters, further confirming that substantial increases in technology investment would be beneficial.

In Panel B, the estimated coefficients confirm a positive relationship between the technology spending of banks and their employment. When too-big-to-fail banks excluded, the estimated parameters for lagged technology spending are positive and statistically significant. They are 0.183 when the dependent variable is number of employees, and 0.190 when the dependent variable is staff expense, as in Column (1) and (2). Both are statistically significant at the $1 \%$ level. In the post financial crisis period, the estimated parameters for previous-year technology spending are also positive $(0.174$ and 0.206 , respectively) and statistically significant at the $1 \%$ level, as in Column (3) and (4). Collectively, the main result remains unchanged when TBTF banks are dropped from the sample and when the post financial crisis period is considered.

\subsection{Conclusions}

Technology is considered as the lifeblood of banks given the rapid advances of technology in the banking industry. Meanwhile, many believe that automation and technology adoption will destroy millions of banking jobs in the future. While the impacts of technology advances in the banking sector are significant, research in this area is limited. The paper fills this gap in the literature by examining the benefit of technology capital in 
bank production and the effect of technology spending on bank employment. This is one of the few empirical studies on the impacts of technology investment on banks.

Based on a sample of U.S. listed commercial banks data from 2000 to 2017, the research first documents strong growth trends in the technology adoption by banks. The median bank technology spending increased $250 \%$, while the median loans and deposits and the number of employees of banks increased by $100 \%$ and $70 \%$, respectively, during the sample period. Then the parameters of a bank value-add production function correcting for endogenous input choices and the measurement errors to assess the returns that banks earn from technology capital is estimated. Technology capital is shown as a major contributing factor to the value-addition generated by banks. On average, technology inputs accounted for an approximately $12.85 \%$ increase in value-added output of U.S. banks. Interestingly, the contribution of technology input becomes stronger after the financial crisis, consistent with the notion that technology has played a more important role in bank production in recent years. These results suggest that technology capital is highly productive and that substantial increases in technology investment would be beneficial.

As the main functions of banks are to collect deposits and make loans, total loans $\&$ deposits and number of branches are used as proxies for bank tasks. Bank employment is measured as the number of employees it employs as well as the staff expense it pays. There is strong evidence that show bank employment and tasks are positively correlated with lagged technology investment. This is consistent with the task-based framework of Acemoglu and Restrepo (2018), which suggest technology can impede employment via its labor-saving effects and enhance employment via its employment-stimulating effects. 
Collectively, the findings illustrate the importance of technology investment to bank productivity and employment. This research also opens the door for additional research on the technology development and adoption of banks and the service industry in general. When technology investment is a necessity, instead of a strategic choice, for firms to succeed in future competition, further research that examines in detail the importance of the components of technology investment concurrent with financing decision, mergers and acquisitions, and corporate governance, management, operational structure and risk may yield considerable insights.

\section{References}

Acemoglu, D. (2002). Technical change, inequality, and the labor market. Journal of Economic Literature, 40(1), 7-72.

Acemoglu, D., \& Autor, D., (2011). Skills, tasks and technologies: Implications for employment and earnings. In Handbook of Labor Economics (Vol. 4, pp. 1043-1171). Elsevier.

Acemoglu, D., \& Restrepo, P., (2017). Robots and jobs: Evidence from US labor markets. NBER Working Paper No. w23285.

Acemoglu, D. \& Restrepo, P. (2018). The race between machine and man: Implications of technology for growth, factor shares and employment. American Economic Review. Forthcoming.

Ackerberg, D., Caves, K., \& Frazer, G. (2006). Structural identification of production functions. Working Paper

Ackerberg, D. A., Caves, K., \& Frazer, G. (2015). Identification properties of recent production function estimators. Econometrica, 83(6), 2411-2451.

Bhattacharya, S., \& Thakor, A. V. (1993). Contemporary banking theory. Journal of Financial Intermediation, 3(1), 2-50. 
Arellano, M., \& Bond, S. (1991). Some tests of specification for panel data: Monte Carlo evidence and an application to employment equations. The Review of Economic Studies, 58(2), 277-297.

Arellano, M., \& Bover, O. (1995). Another look at the instrumental variable estimation of erroR-components models. Journal of Econometrics, 68(1), 29-51.

Autor, D. (2015) "Why Are There Still So Many Jobs? The History and Future of Workplace Automation," Journal of Economic Perspectives, 29(3): 3-30.

Baily, M. N. (1986). What has happened to productivity growth?. Science, 234(4775), 443451.

Basu, S., Inklaar, R., \& Wang, J. C. (2011). The value of risk: measuring the service output of US commercial banks. Economic Inquiry, 49(1), 226-245.

Berger, A. N. (2003). The economic effects of technological progress: Evidence from the banking industry. Journal of Money, Credit, and Banking, 35(2), 141-176.

Berger, A. N., \& Black, L. K. (2011). Bank size, lending technologies, and small business finance. Journal of Banking \& Finance, 35(3), 724-735.

Berger, A. N., \& Humphrey, D. B. (1992). Measurement and efficiency issues in commercial banking. In Output measurement in the service sectors (pp. 245-300). University of Chicago Press.

Berndt, E. R., \& Morrison, C. J. (1995). High-tech capital formation and economic performance in U.S. manufacturing industries an exploratory analysis. Journal of Econometrics, 65(1), 9-43.

Bessen, J. (2015). Learning by doing: the real connection between innovation, wages, and wealth. Yale University Press.

Bessen, J. (2017). Automation and jobs: When technology boosts employment. Boston University. School of Law, Law and Economics Research Paper No. 17-09.

Bloom, N., Sadun, R., \& Van Reenen, J. (2012). Americans do IT better: US multinationals and the productivity miracle. The American Economic Review, 102(1), 167-201.

Blundell, R., \& Bond, S. (1998). Initial conditions and moment restrictions in dynamic panel data models. Journal of Econometrics, 87(1), 115-143.

Blundell, R., \& Bond, S. (2000). GMM estimation with persistent panel data: An application to production functions. Econometric Reviews, 19(3), 321-340. 
Bogliacino, F., \& Pianta, M. (2010). Innovation and employment: a reinvestigation using revised Pavitt classes. Research Policy, 39(6), 799-809.

Bresnahan, T. F. (1999). Computerisation and wage dispersion: an analytical reinterpretation. The Economic Journal, 109(456), 390-415.

Bresnahan, T. F., Brynjolfsson, E., \& Hitt, L. M. (2002). Information technology, workplace organization, and the demand for skilled labor: Firm-level evidence. The Quarterly Journal of Economics, 117(1), 339-376.

Brynjolfsson, E. (1993). The productivity paradox of information technology. Communications of the ACM, 36(12), 66-77.

Brynjolfsson, E., \& Hitt, L. (1995). Information technology as a factor of production: The role of differences among firms. Economics of Innovation and New Technology, 3(3-4), 183-200.

Brynjolfsson, E., \& Hitt, L. (1996). Paradox lost? Firm-level evidence on the returns to information systems spending. Management Science, 42(4), 541-558.

Brynjolfsson, E., \& Hitt, L. M. (2003). Computing productivity: Firm-level evidence. Review of Economics and Statistics, 85(4), 793-808.

Brynjolfsson, E., \& McAfee, A. (2014). The second machine age: Work, progress, and prosperity in a time of brilliant technologies. WW Norton \& Company

Brynjolfsson, E., \& Yang, S. (1996). Information technology and productivity: a review of the literature. Advances in Computers, 43, 179-214.

Charles, K. K., Hurst, E. and Notowidigdo, M. (2013). Manufacturing decline, housing booms, and non-employment. Chicago Booth Research Paper No. 13-57.

Dandapani, K., Lawrence, E. R., \& Rodriguez, J. (2016). Determinants of transactional Internet banking. Journal of Financial Services Research, 1-25.

David, H. (2015). Why are there still so many jobs? The history and future of workplace automation. Journal of Economic Perspectives, 29(3), 3-30.

DeCanio, S. J. (2016). Robots and humans-complements or substitutes?. Journal of Macroeconomics, 49, 280-291.

DeYoung, R. (2010). Banking in the United States. In Oxford Handbook of Banking.

DeYoung, R., Lang, W. W., \& Nolle, D. L. (2007). How the Internet affects output and performance at community banks. Journal of Banking \& Finance, 31(4), 1033-1060. 
Diamond, D. W., \& Dybvig, P. H. (1986). Banking theory, deposit insurance, and bank regulation. The Journal of Business, 59(1), 55-68.

Ford, M. (2015). Rise of the robots: Technology and the threat of a jobless future. Basic Books.

Fort, T. C., Pierce, J. R., \& Schott, P. K. (2018). New perspectives on the decline of US manufacturing employment. Journal of Economic Perspectives, 32(2), 47-72.

Frame, W. S., \& White, L. J. (2014). Technological change, financial innovation, and diffusion in banking. Prepared for The Oxford Handbook of Banking, 2nd Edition. Allen N. Berger, Philip Molyneux, and John O.S. Wilson, eds

Frey, C. B., \& Osborne, M. A. (2017). The future of employment: how susceptible are jobs to computerisation?. Technological Forecasting and Social Change, 114, 254-280.

Gali, J. (1999). Technology, employment, and the business Cycle: Do technology shocks explain aggregate fluctuations?. American Economic Review, 89(1), 249-271.

Goos, M., Manning, A., \& Salomons, A. (2014). Explaining job polarization: Routinebiased technological change and offshoring. American Economic Review, 104(8), 250926.

Greenwood, R., \& Scharfstein, D. (2013). The growth of finance. The Journal of Economic Perspectives, 27(2), 3-28.

Griliches, Z. (1992). Output measurement in the service sectors. University of Chicago Press Griliches, Z. (1994). Productivity, R\&D, and the data constraint. American Economic Review, 84(1), 1-23.

Griliches, Z. \& Mareisse J. (1998). Production functions: The search for identification. In Econometrics and Economic Theory in the Twentieth Century: The Ragnar Prisch Centennial Symposium, 169-203. Cambridge: Cambridge University Press.

Hall, B. H. H., \& Khan, B. (2003). New Economy Handbook, Chapter Adoption of New Technology. Elsevier Science

Hamid, S. S., \& Verma, S. K. (1994). Regional variations in technological change and in potential gains from consolidation among US commercial banks. Journal of Regional Science, 34(3), 411-423.

Hansen, G. D., \& Prescott, E. C. (2002). Malthus to solow. The American Economic Review, 92(4), 1205-1217. 
Harrison, R., Jaumandreu, J., Mairesse, J., \& Peters, B. (2014). Does innovation stimulate employment? A firm-level analysis using comparable micro-data from four European countries. International Journal of Industrial Organization, 35, 29-43.

Hernández-Murillo, R., Llobet, G., \& Fuentes, R. (2010). Strategic online banking adoption. Journal of Banking \& Finance, 34(7), 1650-1663.

Hernando, I., \& Nieto, M. J. (2007). Is the Internet delivery channel changing banks' performance? The case of Spanish banks. Journal of Banking \& Finance, 31(4), 10831099.

Hitt, L. M., \& Brynjolfsson, E. (1996). Productivity, business performance, and consumer surplus: three different measures of information technology value. MIS Quarterly, 121142.

Hood, K. K. (2013). Measuring the services of commercial banks in the national income and products accounts. Survey of Current Business, 93, 8-19.

Hunter, W. C., \& Timme, S. G. (1986). Technical change, organizational form, and the structure of bank production. Journal of Money, Credit and Banking, 18(2), 152-166.

Katz, L. F., \& Murphy, K. M. (1992). Changes in relative wages, 1963-1987: supply and demand factors. The Quarterly Journal of Economics, 107(1), 35-78.

Lee, J., McCullough, J. S., \& Town, R. J. (2013). The impact of health information technology on hospital productivity. The RAND Journal of Economics, 44(3), 545-568.

Levine, O., \& Warusawitharana, M. (2014). Finance and productivity growth: Firm-level evidence. FEDS Working Paper No. 2014-17.

Levinsohn, J., \& Petrin, A. (2003). Estimating production functions using inputs to control for unobservables. The Review of Economic Studies, 70(2), 317-341.

Lichtenberg, F. R. (1995). The output contributions of computer equipment and personnel: A firm-level analysis. Economics of Innovation and New Technology, 3(3-4), 201-218.

Loveman, G. W. (1994). An assessment of the productivity impact of information technologies. Information technology and the corporation of the 1990s: Research studies, 84-110.

Mai, H., Speyer, B., AG, D. B., \& Hoffmann, R. (2012). IT in banks: What does it cost?. Deutsche Bank Research. 
Manyika, J., Chui, M., Bughin, J., Dobbs, R., Bisson, P., \& Marrs, A. (2013). Disruptive technologies: Advances that will transform life, business, and the global economy (Vol. 180). San Francisco, CA: McKinsey Global Institute.

Marschak, J., \& Andrews, W. H. (1944). Random simultaneous equations and the theory of production. Econometrica, 143-205.

Martín-Oliver, A., Ruano, S., \& Salas-Fumás, V. (2013). Why high productivity growth of banks preceded the financial crisis. Journal of Financial Intermediation, 22(4), 688-712.

Martín-Oliver, A., \& Salas-Fumás, V. (2008). The output and profit contribution of information technology and advertising investments in banks. Journal of Financial Intermediation, 17(2), 229-255.

Mokyr, J. (1992). The lever of riches: Technological creativity and economic progress. Oxford University Press.

Morrison, C. J. (1997). Assessing the performance of information technology equipment in U.S. manufacturing industries. Review of Economics and Statistics, 79(3), 471-481.

Olley, G., \& Pakers, A. (1996). The dynamics of productivity in the telecommunications equipment industry. Econometrica, 64(6), 1263-1297.

Petersen, M. A., \& Rajan, R. G. (2002). Does distance still matter? The information revolution in small business lending. The Journal of Finance, 57(6), 2533-2570.

Philippon, T. (2015). Has the US finance industry become less efficient? On the theory and measurement of financial intermediation. The American Economic Review, 105(4), 14081438.

Philippon, T. (2016). The fintech opportunity. NBER Working Paper (No. w22476).

Prasad, B., \& Harker, P. T. (1997). Examining the contribution of information technology toward productivity and performance in U.S. retail banking. The Wharton Financial Institutions Center Working Papers, 97(9).

Rifkin, J. (1996). End of work (pp. 141-157). Pacifica Radio Archives.

Saloner, G., \& Shepard, A. (1995). Adoption of technologies with network Effects: An empirical examination of the adoption of teller machines. RAND Journal of Economics, 26(3), 479-501.

Sealey, C. W., \& Lindley, J. T. (1977). Inputs, outputs, and a theory of production and cost at depository financial institutions. The Journal of Finance, 32(4), 1251-1266. 
Sichel, D. E., \& Oliner, S. D. (2002). Information technology and productivity: Where are we now and where are we going?. Board of Governors of the Federal Reserve System FEDS, 29.

Triplett, J. E., \& Bosworth, B. P. (2004). Productivity in the US Services Sector: New Sources of Economic Growth. Brookings Institution Press.

Triplett, J. E., Bosworth, B. P. (2006). "Baumol's Disease" has been cured: IT and multifactor productivity in US services industries. The New Economy and Beyond: Past, Present, and Future. 34-71.

Van Reenen, J. (1997). Employment and technological innovation: Evidence from UK manufacturing firms. Journal of Labor Economics 15(2), 255-284.

Zeira, J. (1998). Workers, machines, and economic growth. The Quarterly Journal of Economics 113(4), 1091-1117. 
Table 3.1. Summary Statistics

This table reports the summary statistics of regression variables in the sample. All variables are defined in Table A1 in the appendix. To reduce noises in the analysis, firms with fewer than five consecutive years of technology expense and total assets information are excluded. Variables have been winsorized at the $1 \%$ and $99 \%$ tails of the distributions to avoid the influence of extreme observations.

\begin{tabular}{|c|c|c|c|c|c|c|}
\hline & Mean & Median & Std. Dev. & Min & Max & Obs. \\
\hline Market Capitalization $(\$ M)$ & $1,191.36$ & 110.16 & $5,096.43$ & 4.01 & $43,060.26$ & 7,759 \\
\hline Total Asset (\$M) & $7,612.55$ & 965.85 & $31,864.82$ & 97.17 & $268,298.00$ & 8,030 \\
\hline Technology Expense (\$M) & 15.69 & 1.58 & 77.16 & 0.12 & 653.00 & 8,030 \\
\hline Value Added (\$M) & 204.96 & 31.19 & 798.29 & 2.95 & $6,839.70$ & 8,027 \\
\hline Technology Capital - Perpetual (\$M) & 35.06 & 3.39 & 176.17 & 0.18 & $1,516.53$ & 8,030 \\
\hline Conventional Capital - Perpetual (\$M) & $7,329.40$ & 954.60 & $30,366.10$ & 96.58 & $255,449.13$ & 8,030 \\
\hline Technology Capital - Linear (\$M) & 33.67 & 3.31 & 167.82 & 0.18 & $1,429.78$ & 8,030 \\
\hline Conventional Capital - Linear (\$M) & $7,329.50$ & 954.76 & $30,362.91$ & 96.57 & $255,380.50$ & 8,030 \\
\hline Labor $(\$ M)$ & 113.31 & 14.55 & 478.86 & 1.25 & $3,937.00$ & 8,012 \\
\hline Investment (\$M) & 8.62 & 1.59 & 28.28 & -1.73 & 213.00 & 6,380 \\
\hline Loans and Deposits $(\$ M)$ & $9,225.38$ & $1,398.39$ & $34,817.88$ & 133.47 & $280,620.00$ & 8,030 \\
\hline Number of Branches & 57.66 & 15.00 & 174.34 & 1.00 & $1,404.00$ & 7,825 \\
\hline Number of Employees & $1,648.09$ & 264.00 & $6,439.30$ & 26.00 & $52,277.00$ & 7,201 \\
\hline Staff Expense (\$M) & 113.31 & 14.55 & 478.86 & 1.25 & $3,937.00$ & 8,012 \\
\hline Market to Book & 1.33 & 1.21 & 0.65 & 0.18 & 3.64 & 7,758 \\
\hline Leverage & 11.15 & 10.56 & 3.99 & 4.31 & 32.19 & 8,029 \\
\hline Return on Asset (\%) & 0.82 & 0.95 & 0.84 & -3.41 & 2.47 & 7,324 \\
\hline Non-Interest Income $(\%)$ & 21.98 & 20.31 & 12.31 & -2.24 & 68.88 & 8,026 \\
\hline Tier 1 Capital Ratio (\%) & 12.34 & 11.80 & 3.57 & 5.75 & 26.04 & 7,703 \\
\hline
\end{tabular}


Table 3.2. Production Function Parameter Estimates I

This table reports the results from regressions of the natural log of valued-added as the dependent variable on the natural $\log$ of technology capital, which is estimated using a perpetual inventory model, conventional capital, and labor. The standard errors are reported in parentheses. $t$-statistics based on standard errors are in brackets. Significance at the $1 \%, 5 \%$ or $10 \%$ levels is shown with 3,2 , or 1 asterisks, respectively. Production function variables are converted to 2017 dollars using the GDP deflator. The estimation models, which are Olley and Pakes (OP), dynamic panel data (DPD), fixed-effects (FE) and ordinary least squares (OLS), are indicated in the column header. All variables are defined in Table A1 in the appendix. To reduce noises in the analysis, firms with fewer than five consecutive years of technology expense and total assets information are excluded. Variables have been winsorized at the $1 \%$ and $99 \%$ tails of the distributions to avoid the influence of extreme observations.

\begin{tabular}{lcccc}
\hline & $(1)$ & $(2)$ & $(3)$ & $(4)$ \\
Variables & OP & DPD & FE & OLS \\
\hline \multirow{3}{*}{ Technology Capital, $t_{t}$} & $0.085^{* * *}$ & $0.112^{* * *}$ & $0.065^{* * *}$ & $0.067 * * *$ \\
& $(0.030)$ & $(0.032)$ & $(0.009)$ & $(0.007)$ \\
& {$[2.78]$} & {$[3.44]$} & {$[7.25]$} & {$[9.63]$} \\
Conventional Capital, $k_{t}$ & $0.621^{* * *}$ & $0.452^{* * *}$ & $0.371^{* * *}$ & $0.449^{* * *}$ \\
& $(0.038)$ & $(0.025)$ & $(0.011)$ & $(0.010)$ \\
& {$[16.25]$} & {$[17.71]$} & {$[34.39]$} & {$[42.90]$} \\
Labor, $l_{t}$ & $0.253^{* * *}$ & $0.428^{* * *}$ & $0.424^{* * *}$ & $0.435^{* * *}$ \\
& $(0.026)$ & $(0.033)$ & $(0.011)$ & $(0.011)$ \\
& {$[9.74]$} & {$[12.90]$} & {$[37.32]$} & {$[41.13]$} \\
& - & $0.873^{* * *}$ & $0.630^{* * *}$ & $0.926 * * *$ \\
& - & $(0.024)$ & $(0.009)$ & $(0.005)$ \\
Common factor & - & {$[37.04]$} & {$[69.06]$} & {$[200.59]$} \\
$p$-value: $\beta_{t k}+\beta_{c k}+\beta_{l}=1$ & - & 0.000 & 0.000 & 0.000 \\
& 0.285 & 0.812 & 0.000 & 0.000 \\
Observations & & & & \\
Firms & 6,149 & 7,151 & 7,151 & 7,151 \\
\hline
\end{tabular}


Table 3.3. Production Function Parameter Estimates II

This table reports the results from regressions of the natural log of valued-added as the dependent variable on the natural log of technology capital, which is estimated using a linear depreciation schedule, conventional capital, and labor. The standard errors are reported in parentheses. $t$-statistics based on standard errors are in brackets. Significance at the $1 \%, 5 \%$ or $10 \%$ levels is shown with 3,2 , or 1 asterisks, respectively. Production function variables are converted to 2017 dollars using the GDP deflator. The estimation models, which are Olley and Pakes (OP), dynamic panel data (DPD), fixed-effects (FE) and ordinary least squares (OLS), are indicated in the column header. All variables are defined in Table A1 in the appendix. To reduce noises in the analysis, firms with fewer than five consecutive years of technology expense and total assets information are excluded. Variables have been winsorized at the $1 \%$ and $99 \%$ tails of the distributions to avoid the influence of extreme observations.

\begin{tabular}{lcccc}
\hline \multirow{2}{*}{ Variables } & $(1)$ & $(2)$ & $(3)$ & $(4)$ \\
& OP & DPD & FE & OLS \\
Technology Capital, $t_{t}$ & $0.079 * * *$ & $0.083 * * *$ & $0.050^{* * *}$ & $0.053^{* * *}$ \\
& $(0.026)$ & $(0.025)$ & $(0.008)$ & $(0.006)$ \\
Conventional Capital, $k_{t}$ & {$[3.00]$} & {$[3.32]$} & {$[6.29]$} & {$[8.58]$} \\
& $0.638^{* * *}$ & $0.451^{* * *}$ & $0.373 * * *$ & $0.450^{* * *}$ \\
Labor, $l_{t}$ & $(0.036)$ & $(0.026)$ & $(0.011)$ & $(0.010)$ \\
& {$[17.53]$} & {$[17.62]$} & {$[34.48]$} & {$[43.03]$} \\
$\rho$ & $0.253^{* * *}$ & $0.434 * * *$ & $0.427 * * *$ & $0.440 * * *$ \\
& $(0.027)$ & $(0.034)$ & $(0.011)$ & $(0.011)$ \\
& {$[9.47]$} & {$[12.95]$} & {$[37.75]$} & {$[41.70]$} \\
Common factor & - & $0.869 * * *$ & $0.629 * * *$ & $0.925 * * *$ \\
$p$-value: $\beta_{t k}+\beta_{c k}+\beta_{l}=1$ & - & $(0.024)$ & $(0.009)$ & $(0.005)$ \\
& - & {$[36.96]$} & {$[68.83]$} & {$[200.03]$} \\
\hline Observations & - & 0.000 & 0.000 & 0.000 \\
Firms & 0.449 & 0.320 & 0.000 & 0.000 \\
\hline
\end{tabular}


Table 3.4. Persistence in Technology Spending and Employment

Panel A report results from cross-sectional regressions of the natural log of technology and communication expense of banks as the dependent variable on the natural log of their market capitalization (Firm Size) for fthe sample years, as well as a pooled panel regression with year fixed effects. The standard errors are computing using HC3 robust standard errors for the first four columns and are clustered at the firm-level at the last column. The standard errors are reported in parentheses. $t$-statistics based on standard errors are in brackets. Panel B reports cross-sectional regression results for persistence in technology spending and employment of banks. The dependent variables are residual technology spending and employment measures in year $t$, and the independent variables are residual technology spending and employment measures in year $t-1$. The standard errors are clustered at the firm-level and reported in parentheses. $t$-statistics based on robust standard errors are in brackets. Panel C reports correlations of residual technology spending and employment measures. Significance at the $1 \%, 5 \%$ or $10 \%$ levels is shown with 3, 2, or 1 asterisks, respectively. All variables are defined in Table A1 in the appendix. To reduce noises in the analysis, firms with fewer than five consecutive years of technology expense and total assets information are excluded. Variables have been winsorized at the $1 \%$ and $99 \%$ tails of the distributions to avoid the influence of extreme observations.

\begin{tabular}{|c|c|c|c|c|c|}
\hline \multicolumn{6}{|c|}{ Panel A. Technology Spending and Firm Size } \\
\hline & (1) & $(2)$ & $(3)$ & (4) & $(5)$ \\
\hline Variables & 2000 & 2005 & 2010 & 2015 & Pooled \\
\hline \multirow[t]{3}{*}{ Firm Size } & $0.828 * * *$ & $0.855 * * *$ & $0.658 * * *$ & $0.782 * * *$ & $0.767 * * *$ \\
\hline & $(0.027)$ & $(0.027)$ & $(0.029)$ & $(0.024)$ & $(0.022)$ \\
\hline & [31.10] & [31.52] & [22.35] & [32.26] & {$[35.14]$} \\
\hline \multirow[t]{3}{*}{ Constant } & $-3.576^{* * *}$ & $-4.035 * * *$ & $-2.213 * * *$ & $-3.053 * * *$ & $-3.294 * * *$ \\
\hline & $(0.126)$ & $(0.140)$ & $(0.138)$ & $(0.130)$ & $(0.097)$ \\
\hline & {$[-28.28]$} & {$[-28.91]$} & {$[-16.03]$} & {$[-23.41]$} & {$[-34.13]$} \\
\hline Observations & 301 & 426 & 474 & 500 & 7,758 \\
\hline Firms & 301 & 426 & 474 & 500 & 780 \\
\hline$R$-squared & 0.818 & 0.800 & 0.655 & 0.777 & 0.760 \\
\hline Year FE & $\mathrm{NO}$ & $\mathrm{NO}$ & $\mathrm{NO}$ & $\mathrm{NO}$ & YES \\
\hline
\end{tabular}




\begin{tabular}{|c|c|c|c|}
\hline \multicolumn{4}{|c|}{ Panel B. Persistence in Technology Spending and Employment } \\
\hline Variables & $\begin{array}{c}\text { (1) } \\
\text { Residual Technology } \\
\text { Spending }\end{array}$ & $\begin{array}{c}\text { (2) } \\
\text { Residual Number of } \\
\text { Employees }\end{array}$ & $\begin{array}{c}\text { (3) } \\
\text { Residual Staff Expense }\end{array}$ \\
\hline Residual Technology Spending, $t-1$ & $\begin{array}{c}0.905 * * * \\
(0.007) \\
{[135.59]}\end{array}$ & & \\
\hline Residual Number of Employees, $t-1$ & & $\begin{array}{c}0.901 * * * \\
(0.007) \\
{[126.17]}\end{array}$ & \\
\hline Residual Staff Expense, $t-1$ & & & $\begin{array}{c}0.884 * * * \\
(0.008) \\
{[114.41]}\end{array}$ \\
\hline Constant & $\begin{array}{c}0.026 * * * \\
(0.004) \\
{[6.87]}\end{array}$ & $\begin{array}{c}0.020 * * * \\
(0.003) \\
{[6.28]}\end{array}$ & $\begin{array}{c}0.023 * * * \\
(0.003) \\
{[7.19]}\end{array}$ \\
\hline Observations & 6,903 & 6,213 & 6,885 \\
\hline Firms & 780 & 740 & 780 \\
\hline$R$-squared & 0.823 & 0.809 & 0.789 \\
\hline
\end{tabular}




\begin{tabular}{|c|c|c|c|c|c|c|}
\hline \multicolumn{7}{|c|}{ Panel C. Residual Correlations } \\
\hline Variables & $\begin{array}{c}\text { Residual } \\
\text { Technolog } \\
\text { y } \\
\text { Spending, } t\end{array}$ & $\begin{array}{c}\text { Residual } \\
\text { Technolog } \\
\mathrm{y} \\
\text { Spending, } \\
t-1 \\
\end{array}$ & $\begin{array}{c}\text { Residual } \\
\text { Number of } \\
\text { Employees, } \\
t\end{array}$ & $\begin{array}{c}\text { Residual } \\
\text { Number of } \\
\text { Employees, } \\
t-1\end{array}$ & $\begin{array}{l}\text { Residual } \\
\quad \text { Staff } \\
\text { Expense, } t\end{array}$ & $\begin{array}{c}\text { Residual } \\
\text { Staff } \\
\text { Expense, } t- \\
1\end{array}$ \\
\hline Residual Technology Spending, $t$ & 1 & & & & & \\
\hline Residual Technology Spending, $t-1$ & $0.908 * * *$ & 1 & & & & \\
\hline Residual Number of Employees, $t$ & $0.648 * * *$ & $0.579 * * *$ & 1 & & & \\
\hline Residual Number of Employees, $t-1$ & $0.584 * * *$ & $0.644 * * *$ & $0.900 * * *$ & 1 & & \\
\hline Residual Staff Expense, $t$ & $0.672 * * *$ & $0.599 * * *$ & $0.898 * * *$ & $0.808 * * *$ & 1 & \\
\hline Residual Staff Expense, $t-1$ & $0.599 * * *$ & $0.671 * * *$ & $0.798 * * *$ & $0.897 * * *$ & $0.888 * * *$ & 1 \\
\hline
\end{tabular}


Table 3.5. Employment and Technology Spending

This table reports the results from panel regressions where the dependent variables are residual of bank employment and the independent variables are their previous-year residual technology spending as well as other firm characteristics. The coefficients on variables of years are suppressed from reporting. The standard errors are clustered at the firm-level and reported in parentheses. $t$-statistics based on robust standard errors are in brackets. All variables are defined in Table A1 in the appendix. To reduce noises in the analysis, firms with fewer than five consecutive years of technology expense and total assets information are excluded. Variables have been winsorized at the $1 \%$ and $99 \%$ tails of the distributions to avoid the influence of extreme observations.

\begin{tabular}{|c|c|c|c|c|}
\hline Variables & $\begin{array}{c}\text { (1) } \\
\text { Number of } \\
\text { Employees }\end{array}$ & $\begin{array}{c}(2) \\
\text { Staff } \\
\text { Expense } \\
\end{array}$ & $\begin{array}{c}(3) \\
\text { Number of } \\
\text { Employees }\end{array}$ & $\begin{array}{c}(4) \\
\text { Staff } \\
\text { Expense }\end{array}$ \\
\hline Technology Spending, $t-1$ & $\begin{array}{c}0.348 * * * \\
(0.017) \\
{[19.93]}\end{array}$ & $\begin{array}{c}0.380 * * * \\
(0.018) \\
{[21.28]}\end{array}$ & $\begin{array}{c}0.196 * * * \\
(0.023) \\
{[8.49]}\end{array}$ & $\begin{array}{c}0.202 * * * \\
(0.021) \\
{[9.65]}\end{array}$ \\
\hline Market to Book, $t-1$ & & & $\begin{array}{c}-0.196 * * * \\
(0.022) \\
{[-9.00]}\end{array}$ & $\begin{array}{c}-0.227 * * * \\
(0.021) \\
{[-10.73]}\end{array}$ \\
\hline Leverage, $t-1$ & & & $\begin{array}{c}0.015 * * * \\
(0.004) \\
{[4.19]}\end{array}$ & $\begin{array}{c}0.019 * * * \\
(0.003) \\
{[5.70]}\end{array}$ \\
\hline Return on Assets, $t-1$ & & & $\begin{array}{c}-6.813 * * * \\
(1.180) \\
{[-5.77]}\end{array}$ & $\begin{array}{c}-7.910 * * * \\
(1.212) \\
{[-6.53]}\end{array}$ \\
\hline Non-Interest Income, $t-1$ & & & $\begin{array}{c}0.499 * * * \\
(0.108) \\
{[4.63]}\end{array}$ & $\begin{array}{c}0.636 * * * \\
(0.107) \\
{[5.94]}\end{array}$ \\
\hline Tier 1 Capital Ratio, $t-1$ & & & $\begin{array}{c}-0.536 * \\
(0.310) \\
{[-1.73]}\end{array}$ & $\begin{array}{l}-0.267 \\
(0.287) \\
{[-0.93]}\end{array}$ \\
\hline Constant & $\begin{array}{c}-0.036^{*} \\
(0.022) \\
{[-1.65]}\end{array}$ & $\begin{array}{l}-0.029 \\
(0.021) \\
{[-1.42]}\end{array}$ & $\begin{array}{c}0.095 \\
(0.072) \\
{[1.31]}\end{array}$ & $\begin{array}{c}0.042 \\
(0.069) \\
{[0.61]}\end{array}$ \\
\hline Observations & 6,302 & 6,892 & 5,570 & 6,078 \\
\hline Firms & 742 & 780 & 708 & 743 \\
\hline$R$-squared & 0.233 & 0.253 & 0.318 & 0.364 \\
\hline Size $t$ Effects & YES & YES & YES & YES \\
\hline Firm FE & YES & YES & YES & YES \\
\hline Year FE & YES & YES & YES & YES \\
\hline
\end{tabular}


Table 3.6. Tasks and Technology Spending

This table reports the results from panel regressions where the dependent variables are the residual of bank tasks and the independent variables are their previous-year residual technology spending as well as other firm characteristics. The coefficients on variables of years are suppressed from reporting. The standard errors are clustered at the firm-level and reported in parentheses. $t$-statistics based on robust standard errors are in brackets. All variables are defined in Table A1 in the appendix. To reduce noises in the analysis, firms with fewer than five consecutive years of technology expense and total assets information are excluded. Variables have been winsorized at the $1 \%$ and $99 \%$ tails of the distributions to avoid the influence of extreme observations.

\begin{tabular}{|c|c|c|c|c|c|c|}
\hline Variables & $\begin{array}{c}1) \\
\text { Loans \& } \\
\text { Deposits }\end{array}$ & $\begin{array}{c}\text { (2) } \\
\text { Value } \\
\text { Added } \\
\end{array}$ & $\begin{array}{c}\text { (3) } \\
\text { No. of } \\
\text { Branches }\end{array}$ & $\begin{array}{c}(4) \\
\text { Loans \& } \\
\text { Deposits }\end{array}$ & $\begin{array}{c}5) \\
\text { Value } \\
\text { Added } \\
\end{array}$ & $\begin{array}{c}6) \\
\text { No. of } \\
\text { Branches }\end{array}$ \\
\hline Technology Spending, $t-1$ & $\begin{array}{c}0.308 * * * \\
(0.016) \\
{[19.77]}\end{array}$ & $\begin{array}{c}0.301 * * * \\
(0.016) \\
{[18.90]}\end{array}$ & $\begin{array}{c}0.324 * * * \\
(0.017) \\
{[18.74]}\end{array}$ & $\begin{array}{c}0.137 * * * \\
(0.019) \\
{[7.19]}\end{array}$ & $\begin{array}{c}0.161 * * * \\
(0.020) \\
{[7.92]}\end{array}$ & $\begin{array}{c}0.191 * * * \\
(0.022) \\
{[8.56]}\end{array}$ \\
\hline Market to Book, $t-1$ & & & & $\begin{array}{c}-0.230 * * * \\
(0.019) \\
{[-12.40]}\end{array}$ & $\begin{array}{c}-0.199 * * * \\
(0.019) \\
{[-10.30]}\end{array}$ & $\begin{array}{c}-0.171 * * * \\
(0.021) \\
{[-8.04]}\end{array}$ \\
\hline Leverage, $t-1$ & & & & $\begin{array}{c}0.020 * * * \\
(0.003) \\
{[5.83]}\end{array}$ & $\begin{array}{c}0.019 * * * \\
(0.004) \\
{[5.12]}\end{array}$ & $\begin{array}{c}0.014 * * * \\
(0.003) \\
{[4.96]}\end{array}$ \\
\hline Return on Assets, $t-1$ & & & & $\begin{array}{c}-5.412 * * * \\
(1.105) \\
{[-4.90]}\end{array}$ & $\begin{array}{c}-3.430 * * * \\
(1.153) \\
{[-2.97]}\end{array}$ & $\begin{array}{c}-6.028 * * * \\
(1.084) \\
{[-5.56]}\end{array}$ \\
\hline Non-Interest Income, $t-1$ & & & & $\begin{array}{c}-0.208 * * \\
(0.087) \\
{[-2.39]}\end{array}$ & $\begin{array}{c}-0.307 * * * * \\
(0.090) \\
{[-3.40]}\end{array}$ & $\begin{array}{l}-0.046 \\
(0.100) \\
{[-0.46]}\end{array}$ \\
\hline Tier 1 Capital Ratio, $t-1$ & & & & $\begin{array}{c}-1.313 * * * \\
(0.277) \\
{[-4.75]}\end{array}$ & $\begin{array}{c}-1.054 * * * \\
(0.303) \\
{[-3.48]}\end{array}$ & $\begin{array}{l}-0.337 \\
(0.285) \\
{[-1.18]}\end{array}$ \\
\hline Constant & $\begin{array}{c}-0.034^{*} \\
(0.018) \\
{[-1.87]}\end{array}$ & $\begin{array}{l}-0.023 \\
(0.017) \\
{[-1.35]}\end{array}$ & $\begin{array}{l}-0.023 \\
(0.022) \\
{[-1.04]}\end{array}$ & $\begin{array}{c}0.284 * * * \\
(0.069) \\
{[4.11]}\end{array}$ & $\begin{array}{c}0.243 * * * \\
(0.072) \\
{[3.37]}\end{array}$ & $\begin{array}{c}0.134 * * \\
(0.061) \\
{[2.19]}\end{array}$ \\
\hline Observations & 6,903 & 6,903 & 6,753 & 6,086 & 6,086 & 5,974 \\
\hline Firms & 780 & 780 & 774 & 743 & 743 & 737 \\
\hline$R$-squared & 0.203 & 0.196 & 0.228 & 0.323 & 0.287 & 0.303 \\
\hline Size $t$ Effects & YES & YES & YES & YES & YES & YES \\
\hline Firm FE & YES & YES & YES & YES & YES & YES \\
\hline Year FE & YES & YES & YES & YES & YES & YES \\
\hline
\end{tabular}


Table 3.7. Robustness Checks

This table presents the results of the robustness checks. Panel A reports the production function parameter estimates with technology capital estimated using a perpetual inventory model and production variables converted to 2017 dollars using the GDP deflator on the too-big-to-fail banks excluded subsample and on the post financial crisis period (2010-2017) subsample. The estimation models, which are Olley and Pakes (OP), dynamic panel data (DPD), fixed-effects (FE) and ordinary least squares (OLS), are indicated in the column header. Panel B reports the results from panel regressions of employment and tasks on technology spending on the too-bigto-fail banks excluded subsample and on the post financial crisis period (2010-2017) subsample. The dependent variables are indicated in the column header. The standard errors are clustered at the firm-level and reported in parentheses. $t$-statistics based on robust standard errors are in brackets. Significance at the 1\%, 5\% or 10\% levels is shown with 3, 2, or 1 asterisks, respectively. All variables are defined in Table A1 in the appendix. To reduce noises in the analysis, firms with fewer than five consecutive years of technology expense and total assets information are excluded. Variables have been winsorized at the $1 \%$ and $99 \%$ tails of the distributions to avoid the influence of extreme observations.

\begin{tabular}{|c|c|c|c|c|c|c|c|c|}
\hline \multicolumn{9}{|c|}{ Panel A. Production Function Parameter Estimates } \\
\hline & \multicolumn{4}{|c|}{ Too-Big-To-Fail Banks Excluded } & \multicolumn{4}{|c|}{ Post Financial Crisis Period (2010-2017) } \\
\hline & (1) & (2) & (3) & (4) & $(5)$ & (6) & (7) & (8) \\
\hline Variables & $\mathrm{OP}$ & DPD & FE & OLS & OP & DPD & $\mathrm{FE}$ & OLS \\
\hline \multirow[t]{3}{*}{ Technology Capital, $t_{t}$} & $0.065^{* *}$ & $0.114 * * *$ & $0.064 * * *$ & $0.070 * * *$ & $0.131 * * *$ & $0.239 * * *$ & $0.133 * * *$ & $0.107 * * *$ \\
\hline & $(0.032)$ & $(0.033)$ & $(0.009)$ & $(0.007)$ & $(0.044)$ & $(0.060)$ & $(0.014)$ & $(0.010)$ \\
\hline & [2.05] & [3.47] & [7.07] & [9.94] & [2.99] & [4.00] & [9.73] & {$[11.00]$} \\
\hline \multirow[t]{3}{*}{ Conventional Capital, $k_{t}$} & $0.600 * * *$ & $0.443 * * *$ & $0.369 * * *$ & $0.443 * * *$ & $0.631 * * *$ & $0.357 * * *$ & $0.307 * * *$ & $0.422 * * *$ \\
\hline & $(0.048)$ & $(0.025)$ & $(0.011)$ & $(0.011)$ & $(0.052)$ & $(0.042)$ & $(0.015)$ & $(0.014)$ \\
\hline & [12.61] & [17.56] & [33.71] & [41.96] & [12.11] & [8.45] & [20.80] & [29.99] \\
\hline \multirow[t]{3}{*}{ Labor, $l_{t}$} & $0.264 * * *$ & $0.435 * * *$ & $0.425 * * *$ & $0.440 * * *$ & $0.194 * * *$ & $0.461 * * *$ & $0.394 * * *$ & $0.463 * * *$ \\
\hline & $(0.024)$ & $(0.034)$ & $(0.012)$ & $(0.011)$ & $(0.032)$ & $(0.060)$ & $(0.017)$ & $(0.015)$ \\
\hline & [11.08] & [12.96] & [36.84] & [41.18] & [6.12] & [7.69] & [23.10] & [29.92] \\
\hline \multirow[t]{3}{*}{$\rho$} & - & $0.872 * * *$ & $0.630 * * *$ & $0.915 * * *$ & - & $0.863 * * *$ & $0.458 * * *$ & $0.926 * * *$ \\
\hline & - & $(0.023)$ & $(0.009)$ & $(0.005)$ & - & $(0.053)$ & $(0.014)$ & $(0.006)$ \\
\hline & - & [37.60] & [67.56] & [187.13] & - & [16.41] & [32.13] & [151.80] \\
\hline
\end{tabular}




\begin{tabular}{|c|c|c|c|c|c|c|c|c|}
\hline $\begin{array}{l}\text { Common factor } \\
p \text {-value: } \beta_{t k}+\beta_{c k}+\beta_{l}=1\end{array}$ & 0.098 & $\begin{array}{l}0.000 \\
0.809\end{array}$ & $\begin{array}{l}0.000 \\
0.000\end{array}$ & $\begin{array}{l}0.000 \\
0.000\end{array}$ & 0.353 & $\begin{array}{l}0.000 \\
0.394\end{array}$ & $\begin{array}{l}0.000 \\
0.000\end{array}$ & $\begin{array}{l}0.000 \\
0.593\end{array}$ \\
\hline $\begin{array}{l}\text { Observations } \\
\text { Firms }\end{array}$ & $\begin{array}{c}6,013 \\
749\end{array}$ & $\begin{array}{c}6,922 \\
758\end{array}$ & $\begin{array}{c}6,922 \\
758\end{array}$ & $\begin{array}{c}6,922 \\
758\end{array}$ & $\begin{array}{c}3,711 \\
595\end{array}$ & $\begin{array}{c}3,326 \\
582\end{array}$ & $\begin{array}{c}3,326 \\
582\end{array}$ & $\begin{array}{c}3,326 \\
582\end{array}$ \\
\hline \multicolumn{9}{|c|}{ Panel B. Employment and Technology Spending } \\
\hline & \multicolumn{4}{|c|}{ Too-Big-To-Fail Banks Excluded } & \multicolumn{4}{|c|}{ Post Financial Crisis Period (2010-2017) } \\
\hline Variables & \multicolumn{2}{|c|}{$\begin{array}{c}\text { (1) } \\
\text { Number of } \\
\text { Employees } \\
\end{array}$} & \multicolumn{2}{|c|}{ Staff Expense } & \multicolumn{2}{|c|}{$\begin{array}{l}\text { Number of } \\
\text { Employees }\end{array}$} & \multicolumn{2}{|c|}{ Staff Expense } \\
\hline Technology Spending, $t-1$ & \multicolumn{2}{|c|}{$\begin{array}{c}0.183 * * * \\
(0.023) \\
{[7.86]}\end{array}$} & \multicolumn{2}{|c|}{$\begin{array}{c}0.190 * * * \\
(0.021) \\
{[8.97]}\end{array}$} & \multicolumn{2}{|c|}{$\begin{array}{c}0.174 * * * \\
(0.030) \\
{[5.82]}\end{array}$} & \multicolumn{2}{|c|}{$\begin{array}{c}0.206 * * * \\
(0.029) \\
{[7.18]}\end{array}$} \\
\hline 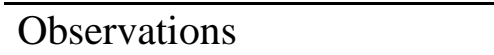 & \multicolumn{2}{|c|}{5,393} & \multicolumn{2}{|c|}{5,899} & \multicolumn{2}{|c|}{2,585} & \multicolumn{2}{|c|}{2,947} \\
\hline Firms & \multicolumn{2}{|c|}{687} & \multicolumn{2}{|c|}{722} & \multicolumn{2}{|c|}{490} & \multicolumn{2}{|c|}{543} \\
\hline$R$-squared & \multicolumn{2}{|c|}{0.325} & \multicolumn{2}{|c|}{0.368} & \multicolumn{2}{|c|}{0.200} & \multicolumn{2}{|c|}{0.237} \\
\hline Controls & \multicolumn{2}{|c|}{ YES } & \multicolumn{2}{|c|}{ YES } & \multicolumn{2}{|c|}{ YES } & \multicolumn{2}{|c|}{ YES } \\
\hline Size $t$ Effects & \multicolumn{2}{|c|}{ YES } & \multicolumn{2}{|c|}{ YES } & \multicolumn{2}{|c|}{ YES } & \multicolumn{2}{|c|}{ YES } \\
\hline Firm FE & \multicolumn{2}{|c|}{ YES } & \multicolumn{2}{|c|}{ YES } & \multicolumn{2}{|c|}{ YES } & \multicolumn{2}{|c|}{ YES } \\
\hline Year FE & & & & & & & & \\
\hline
\end{tabular}




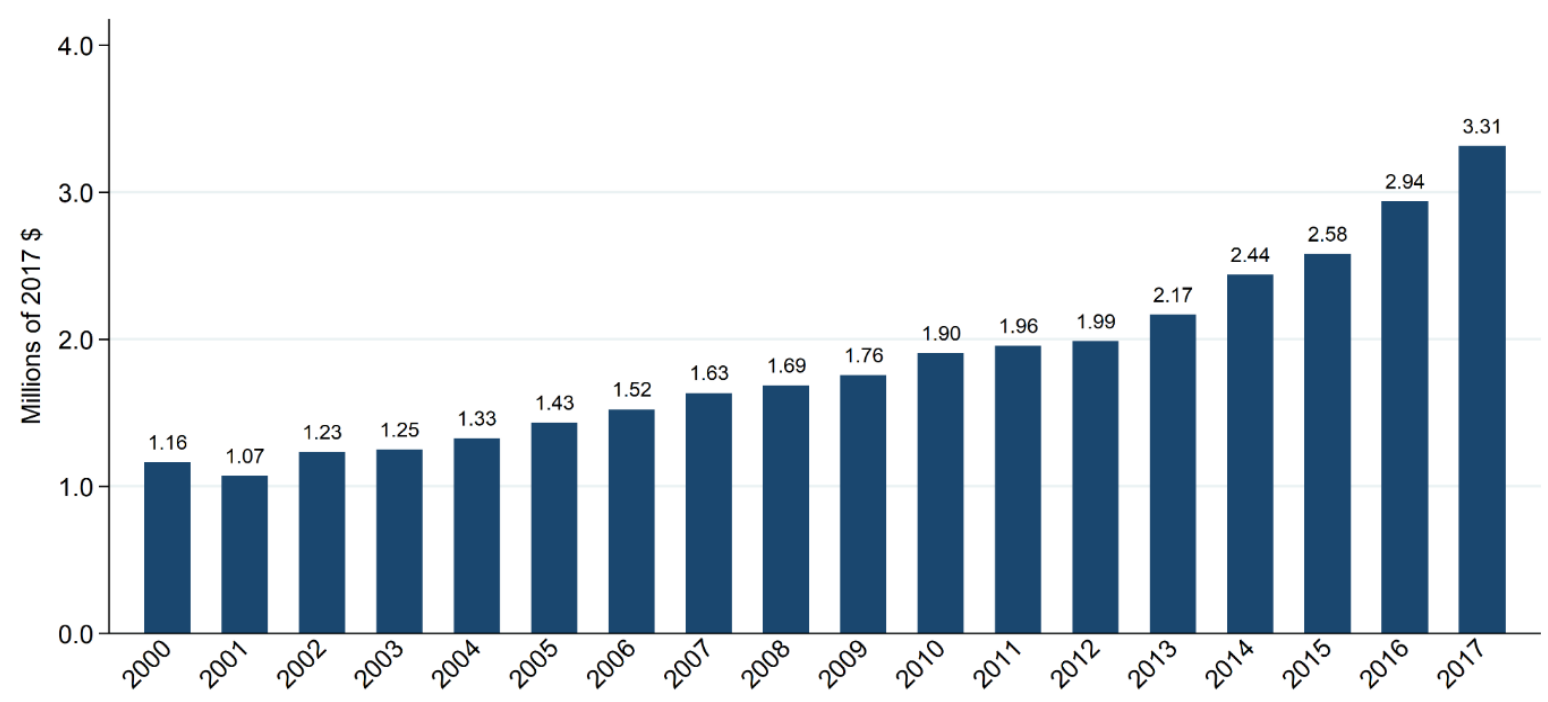

Figure 3.1. Technology Spending Trends

This figure illustrates the trends (medians) of technology and communication expense of banks in the sample during 2000-2017. Technology and communication expense is converted to 2017 dollars using the GDP deflator. All variables are defined in Table A1 in the appendix. To reduce noises in the analysis, firms with fewer than five consecutive years of technology expense and total assets information are excluded. Variables have been winsorized at the $1 \%$ and $99 \%$ tails of the distributions to avoid the influence of extreme observation 


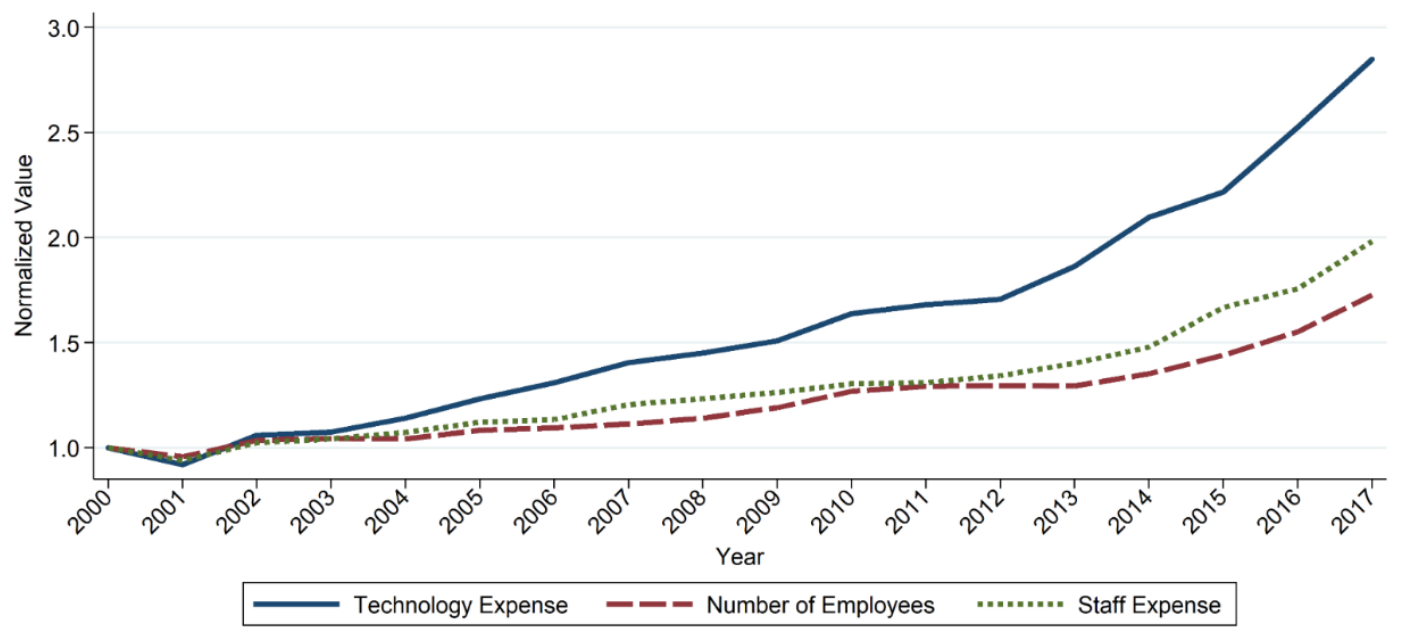

Figure 3.2. Employment and Technology Spending Over Time

This figure illustrates the evolution of the median of technology and communication expense, the number of employees and staff expense of banks in the sample during 20002017. All values are normalized to equal one in the year 2000. Monetary variables are converted to 2017 dollars using the GDP deflator. All variables are defined in Table A1 in the appendix. To reduce noises in the analysis, firms with fewer than five consecutive years of technology expense and total assets information are excluded. Variables have been winsorized at the $1 \%$ and $99 \%$ tails of the distributions to avoid the influence of extreme observations. 

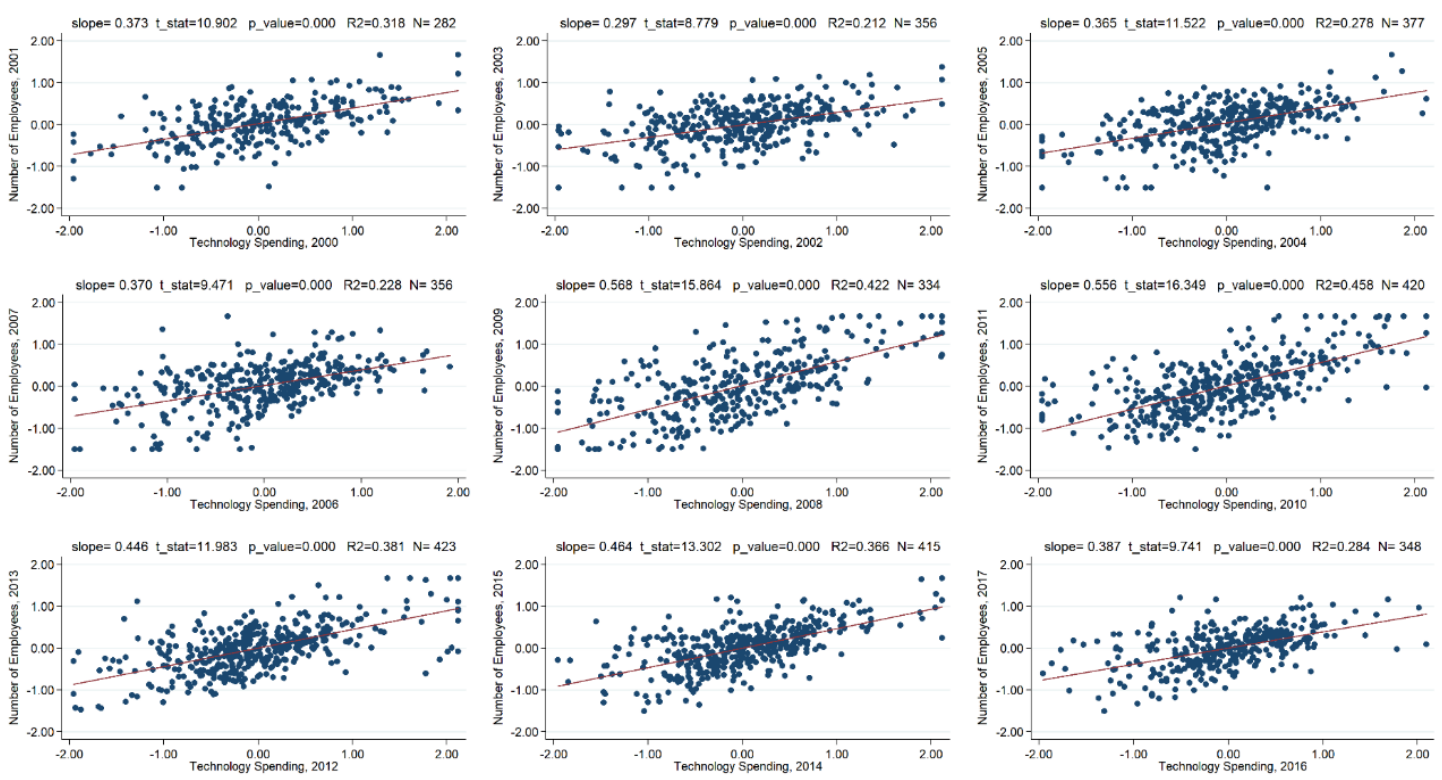

Figure 3.3. Number of Employees and Technology Spending in the Cross-Sections

This figure plots residual number of employees on the vertical axis against residual technology spending on the horizontal axis for nine sample years. The $t$-statistics are calculated using HC3-robust standard errors with an adjustment to account for the degrees of freedom absorbed by computing residuals. All variables are defined in Table A1 in the appendix. To reduce noises in the analysis, firms with fewer than five consecutive years of technology expense and total assets information are excluded. Variables have been winsorized at the $1 \%$ and $99 \%$ tails of the distributions to avoid the influence of extreme observations. 

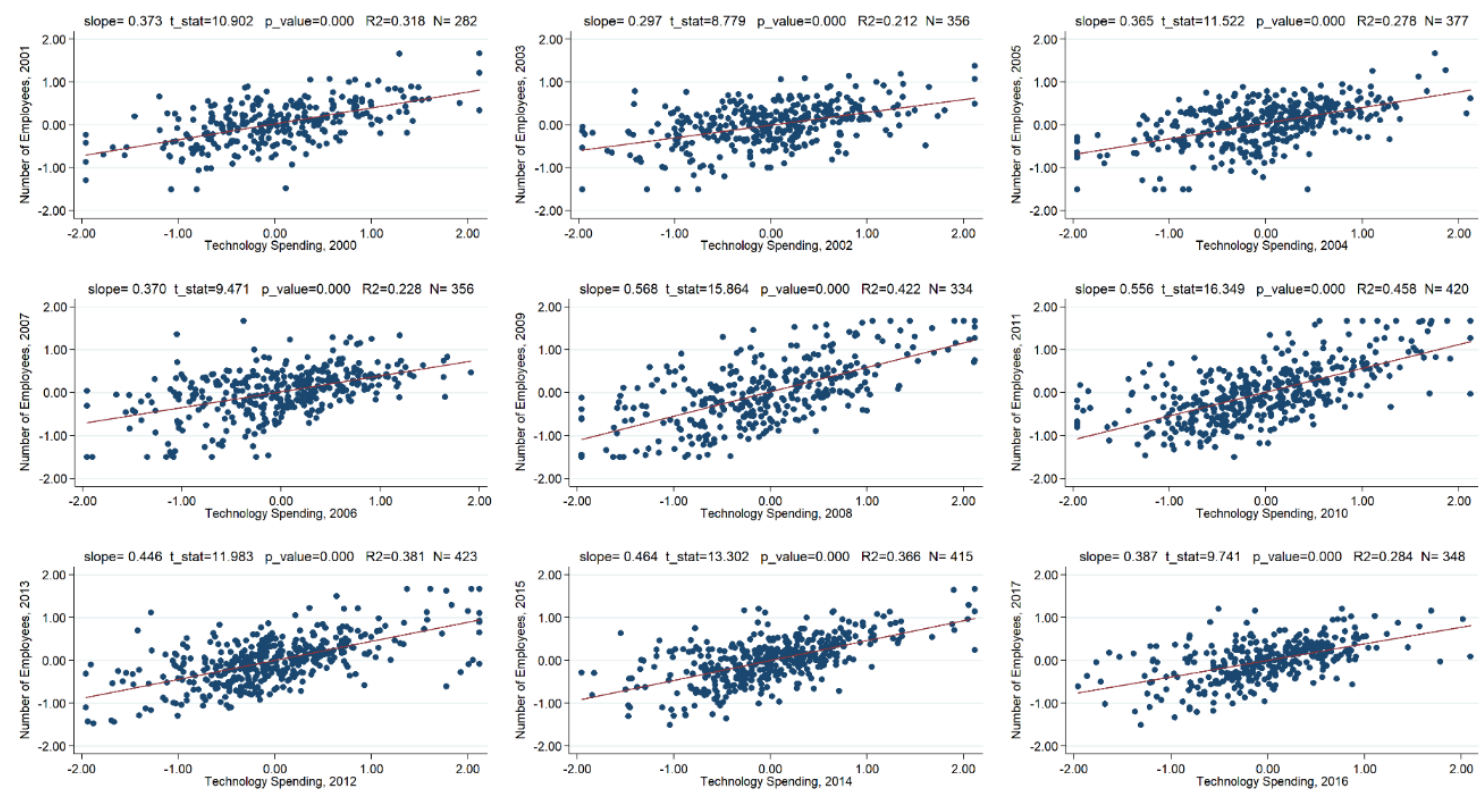

Figure 3.4. Staff Expense and Technology Spending in the Cross-Sections

This figure plots residual staff expense on the vertical axis against residual technology spending on the horizontal axis for nine sample years. The $t$-statistics are calculated using HC3-robust standard errors with an adjustment to account for the degrees of freedom absorbed by computing residuals. All variables are defined in Table A1 in the appendix. To reduce noises in the analysis, firms with fewer than five consecutive years of technology expense and total assets information are excluded. Variables have been winsorized at the $1 \%$ and $99 \%$ tails of the distributions to avoid the influence of extreme observations. 
Appendix

Table A1. Definition of Variables

\begin{tabular}{|c|c|c|}
\hline Variable & Abb. & Definition \\
\hline $\begin{array}{l}\text { Technology } \\
\text { and } \\
\text { Communicati } \\
\text { on Expense }\end{array}$ & $\begin{array}{l}\text { Technology } \\
\text { Expense or } \\
\text { Technology } \\
\text { Spending }\end{array}$ & $\begin{array}{l}\text { Expenses paid for communications, data processing } \\
\text { and technology such as computers, software, } \\
\text { information systems and telecommunications, as } \\
\text { defined by S\&P Global Market Intelligence (SNL } \\
\text { Financial). (SNL Keyfield: } 132659 \text {, } \\
\text { tech_comm_exp) }\end{array}$ \\
\hline $\begin{array}{l}\text { Valued- } \\
\text { Added }\end{array}$ & Valued-added & Net interest income (Compustat: niint) \\
\hline $\begin{array}{l}\text { Technology } \\
\text { Capital }\end{array}$ & $\begin{array}{l}\text { Technology } \\
\text { capital }\end{array}$ & $\begin{array}{l}\text { Technology capital is constructed using a perpetual } \\
\text { inventory model with a depreciation rate of } 35 \% \text { or a } \\
\text { four-year linear depreciation schedule. }\end{array}$ \\
\hline $\begin{array}{l}\text { Conventional } \\
\text { Capital } \\
\text { Labor }\end{array}$ & $\begin{array}{l}\text { Conventional } \\
\text { capital } \\
\text { Labor }\end{array}$ & $\begin{array}{l}\text { Total assets (Compustat: at) minus intangible assets } \\
\text { (Compustat: intan) and technology capital } \\
\text { Staff expense (Compustat: xlr). It represents salaries, } \\
\text { wages, pension costs, profit sharing and incentive } \\
\text { compensation, payroll taxes, and other employee } \\
\text { benefits. }\end{array}$ \\
\hline $\begin{array}{l}\text { Investment } \\
\text { Expenditure }\end{array}$ & Investment & $\begin{array}{l}\text { Capital expenditure (Compustat: capx) minus sale of } \\
\text { property (Compustat: sppe). Sppe is set to zero if } \\
\text { missing. }\end{array}$ \\
\hline $\begin{array}{l}\text { Number of } \\
\text { Automatic } \\
\text { Teller } \\
\text { Machines }\end{array}$ & ATMs & $\begin{array}{l}\text { The number of automatic teller machines operated. } \\
\text { [SNL Keyfield: } 131225 \text {, num_atms] }\end{array}$ \\
\hline Residual & Res & $\begin{array}{l}\text { Residuals obtained from the regression model: } \\
\text { Variable }_{i, t}=\alpha+\beta_{1} \text { LnSize }_{i, t}+\varepsilon_{i} . \text { Where } \\
\text { Variable are the natural log of technology and } \\
\text { communication expense, employment measures, } \\
\text { tasks measures, respectively, and } L n S i z e \text { is the } \\
\text { natural log of market capitalization at the end of the } \\
\text { fiscal year. }\end{array}$ \\
\hline $\begin{array}{l}\text { Number of } \\
\text { Employees }\end{array}$ & $\begin{array}{l}\text { Number of } \\
\text { Employees }\end{array}$ & $\begin{array}{l}\text { The number of people employed by the company } \\
\text { (Compustat: emp) }\end{array}$ \\
\hline Staff Expense & Staff Expense & Staff expense of the company (Compustat: xlr) \\
\hline $\begin{array}{l}\text { Total Loans } \\
\text { and Deposits }\end{array}$ & $\begin{array}{l}\text { Loans and } \\
\text { Deposits }\end{array}$ & $\begin{array}{l}\text { The sum of total loans (Compustat: lntal) and total } \\
\text { deposits (Compustat: dptc). }\end{array}$ \\
\hline $\begin{array}{l}\text { Number of } \\
\text { Branches }\end{array}$ & $\begin{array}{l}\text { Number of } \\
\text { Branches }\end{array}$ & $\begin{array}{l}\text { For banks and thrifts, the number of offices a } \\
\text { company operates within the United States, updated } \\
\text { for completed M\&A activity. A branch/office is any } \\
\text { location, or facility, of a financial institution, } \\
\text { including its main office, where deposit accounts are } \\
\text { opened, deposits are accepted, checks paid, and }\end{array}$ \\
\hline
\end{tabular}




\begin{tabular}{|c|c|c|}
\hline Firm Size & Firm Size & $\begin{array}{l}\text { [SNL KeyField: } 131227 \text {, total_numfices] } \\
\text { The natural log of market capitalization at the end of } \\
\text { the fiscal year (Compustat: prcc_f*csho). }\end{array}$ \\
\hline Market to & Market to & The ratio of the market capitalization of equity \\
\hline Book & Book & $\begin{array}{l}\text { (Compustat: prcc_f*csho) to book value of equity } \\
\text { (Compustat: ceq+txdb). txdb is set to zero if missing. }\end{array}$ \\
\hline Leverage & Leverage & $\begin{array}{l}\text { The ratio of total assets (Compustat: at) to book } \\
\text { value of equity (Compustat: ceq+txdb). Txdb is set } \\
\text { to zero if missing. }\end{array}$ \\
\hline $\begin{array}{l}\text { Return on } \\
\text { Assets }\end{array}$ & ROA & $\begin{array}{l}\text { Earnings before extraordinary items (Compustat: ib) } \\
\text { plus depreciation and amortization (Compustat: dp) } \\
\text { to total assets (Compustat: at). }\end{array}$ \\
\hline $\begin{array}{l}\text { Non-Interest } \\
\text { Income }\end{array}$ & $\begin{array}{l}\text { Non-Interest } \\
\text { Income }\end{array}$ & $\begin{array}{l}\text { The ratio of banks' Non-Interest Income } \\
\text { (Compustat: tnii) to the sum of Net Interest Income } \\
\text { (Compustat: niint) and Non-Interest Income } \\
\text { (Compustat: tnii) }\end{array}$ \\
\hline $\begin{array}{l}\text { Tier } 1 \text { Capital } \\
\text { Ratio }\end{array}$ & $\begin{array}{l}\text { Tier } 1 \text { Capital } \\
\text { Ratio }\end{array}$ & $\begin{array}{l}\text { Risk-adjusted capital ratio - Tier1 (Compustat: } \\
\text { capr1) }\end{array}$ \\
\hline $\begin{array}{l}\text { Too-big-to- } \\
\text { fail banks }\end{array}$ & TBTF Banks & $\begin{array}{l}\text { Banks whose gross total assets exceed } \$ 50 \text { billion in } \\
2017 \text { dollars. Gross total assets are the sum of total } \\
\text { assets (Compustat: at) and provision for loan losses } \\
\text { (Compustat: pclc). pclc is set to zero if missing. }\end{array}$ \\
\hline
\end{tabular}


Table A2. Residual Employment and Tasks

This table report results from cross-sectional regressions of bank employment and tasks, respectively, as the dependent variable on the natural log of their market capitalization (Firm Size) for fthe sample years, as well as a pooled panel regression with year fixed effects. The standard errors are reported in parentheses. $t$-statistics are in brackets. The standard errors are computing using HC3 robust standard errors for the fthe sample years and are clustered at the firm-level at the pooled panel regressions. Significance at the $1 \%, 5 \%$ or $10 \%$ levels is shown with 3,2 , or 1 asterisks, respectively. All variables are defined in Table A1 in the appendix. To reduce noises in the analysis, firms with fewer than five consecutive years of technology expense and total assets information are excluded. Variables have been winsorized at the $1 \%$ and $99 \%$ tails of the distributions to avoid the influence of extreme observations.

\section{Panel A. Employment and Firm Size}

\begin{tabular}{|c|c|c|c|c|c|c|c|c|c|c|}
\hline & \multicolumn{5}{|c|}{ Number of Employees } & \multicolumn{5}{|c|}{ Staff Expense } \\
\hline & (1) & (2) & (3) & (4) & (5) & (6) & (7) & (8) & (9) & $(10)$ \\
\hline Variables & 2000 & 2005 & 2010 & 2015 & Pooled & 2000 & 2005 & 2010 & 2015 & Pooled \\
\hline \multirow[t]{3}{*}{ Firm Size } & $0.788 * * *$ & $0.858 * * *$ & $0.636 * * *$ & $0.764 * * *$ & $0.758 * * *$ & $0.818 * * *$ & $0.899 * * *$ & $0.684 * * *$ & $0.818 * * *$ & $0.799 * * *$ \\
\hline & $(0.018)$ & $(0.016)$ & $(0.024)$ & $(0.022)$ & $(0.015)$ & $(0.018)$ & $(0.016)$ & $(0.023)$ & $(0.017)$ & $(0.014)$ \\
\hline & {$[44.21]$} & {$[53.40]$} & [26.73] & {$[35.14]$} & {$[50.95]$} & [45.09] & {$[56.34]$} & [30.10] & [48.33] & {$[55.90]$} \\
\hline \multirow[t]{3}{*}{ Constant } & $2.080 * * *$ & $1.229 * * *$ & $2.867 * * *$ & $1.743 * * *$ & $2.220 * * *$ & $-1.186 * * *$ & $-1.882 * * *$ & -0.132 & $-1.121 * * *$ & $-1.097 * * *$ \\
\hline & $(0.092)$ & $(0.088)$ & $(0.119)$ & $(0.121)$ & $(0.071)$ & $(0.093)$ & $(0.086)$ & $(0.112)$ & $(0.092)$ & $(0.069)$ \\
\hline & [22.57] & [13.97] & [24.09] & {$[14.40]$} & [31.04] & {$[-12.71]$} & {$[-21.86]$} & {$[-1.18]$} & {$[-12.18]$} & {$[-15.87]$} \\
\hline Observations & 277 & 404 & 438 & 425 & 7,052 & 300 & 425 & 474 & 499 & 7,744 \\
\hline Firms & 277 & 404 & 438 & 425 & 747 & 300 & 425 & 474 & 499 & 780 \\
\hline$R$-squared & 0.889 & 0.885 & 0.730 & 0.847 & 0.827 & 0.900 & 0.913 & 0.768 & 0.893 & 0.858 \\
\hline Year FE & NO & NO & NO & $\mathrm{NO}$ & YES & NO & NO & NO & $\mathrm{NO}$ & YES \\
\hline
\end{tabular}




\begin{tabular}{|c|c|c|c|c|c|c|c|c|c|c|c|c|c|c|c|}
\hline \multicolumn{16}{|c|}{ Panel B. Tasks and Firm Size } \\
\hline & \multicolumn{5}{|c|}{ Loans and Deposits } & \multicolumn{5}{|c|}{ Value Added } & \multicolumn{5}{|c|}{ Number of Branches } \\
\hline \multirow[b]{2}{*}{ Variables } & (1) & (2) & (3) & (4) & (5) & (6) & (7) & (8) & (9) & (10) & (11) & (12) & (13) & (14) & $(15)$ \\
\hline & 2000 & 2005 & 2010 & 2015 & $\begin{array}{c}\text { Pool } \\
\text { ed }\end{array}$ & 2000 & 2005 & 2010 & 2015 & $\begin{array}{c}\text { Pool } \\
\text { ed }\end{array}$ & 2000 & 2005 & 2010 & 2015 & $\begin{array}{c}\text { Pool } \\
\text { ed }\end{array}$ \\
\hline \multirow{3}{*}{ Firm Size } & 0.800 & $.881 *$ & 665 & .847 & .793 & 812 & 895 & $687^{*}$ & $.834^{\prime}$ & $805^{*}$ & $.655^{*}$ & $0.727 *$ & 0.534 & .000 & . \\
\hline & $* *$ & $* *$ & $* *$ & $* *$ & $* *$ & $* *$ & $* *$ & $* *$ & $* *$ & $* *$ & $* *$ & $* *$ & $* *$ & $* *$ & $* *$ \\
\hline & $\begin{array}{l}(0.015) \\
{[52.10]}\end{array}$ & {$\left[\begin{array}{l}0.011 \\
80.21\end{array}\right.$} & {$\left[\begin{array}{l}0.020 \\
33.10\end{array}\right.$} & $\begin{array}{l}(0.013) \\
{[65.76]}\end{array}$ & $\begin{array}{l}(0.010) \\
{[75.74]}\end{array}$ & $\begin{array}{l}(0.016) \\
{[50.08]}\end{array}$ & $\begin{array}{l}(0.012) \\
{[75.53]}\end{array}$ & $0.019)$ & $\begin{array}{l}0.012) \\
67.57]\end{array}$ & $\begin{array}{l}0.010)(78.02] \\
0\end{array}$ & $\begin{array}{l}(0.047) \\
14.08]\end{array}$ & $\begin{array}{l}(0.024) \\
{[29.82]}\end{array}$ & $\begin{array}{l}0.023) \\
23.60]\end{array}$ & $1<0$. & . 01$]$ \\
\hline \multirow[t]{3}{*}{ Constant } & $\begin{array}{c}3.481 * \\
* *\end{array}$ & $\begin{array}{c}2.821 * \\
* *\end{array}$ & $\begin{array}{c}4.540^{*} \\
* *\end{array}$ & $\begin{array}{c}3.326 \\
* *\end{array}$ & $\begin{array}{c}3.511 \\
* *\end{array}$ & $\begin{array}{c}- \\
0.288^{*} \\
* *\end{array}$ & $\begin{array}{c}- \\
1.037 * \\
* *\end{array}$ & $\begin{array}{c}0.660 * \\
* *\end{array}$ & $\begin{array}{c}- \\
0.481 * \\
* *\end{array}$ & $\begin{array}{c}- \\
0.257 * \\
* *\end{array}$ & -0.247 & $\begin{array}{c}- \\
0.941 * \\
* *\end{array}$ & $\begin{array}{c}0.499 * \\
* *\end{array}$ & $\begin{array}{c}- \\
0.564 * \\
* *\end{array}$ & $\begin{array}{c}- \\
0.255^{*} \\
* *\end{array}$ \\
\hline & \multirow{2}{*}{\multicolumn{15}{|c|}{$\begin{array}{l}(0.074)(0.059)(0.102)(0.073)(0.051)(0.075)(0.063)(0.097)(0.070)(0.050)(0.189)(0.122)(0.114)(0.124)(0.079) \\
{[47.23][47.44][44.43][45.43][68.44][-3.81]_{16.46]}^{[-}[6.84][-6.89][-5.18][-1.31][-7.69][4.39][-4.55][-3.22]}\end{array}$}} \\
\hline & & & & & & & & & & & & & & & \\
\hline Obs & 301 & 426 & 474 & 500 & $\begin{array}{c}7,75 \\
8\end{array}$ & 301 & 426 & 474 & 500 & $\begin{array}{c}7,75 \\
7\end{array}$ & 283 & 417 & 468 & 489 & $\begin{array}{c}7,57 \\
9\end{array}$ \\
\hline Firms & 301 & 426 & 474 & 500 & 780 & 301 & 426 & 474 & 500 & 780 & 283 & 417 & 468 & 489 & 775 \\
\hline \multirow{2}{*}{$R$-squared } & 0.92 & 0.95 & 0.78 & 0.94 & 0.89 & 0.93 & 0.95 & 0.80 & 0.93 & 0.90 & 0.68 & 0.72 & 0.60 & 0.69 & 0.68 \\
\hline & 9 & 0 & 2 & 1 & 3 & 7 & 5 & 7 & 9 & 2 & 6 & 4 & 5 & 5 & 6 \\
\hline Year FE & $\mathrm{NO}$ & NO & NO & $\mathrm{NO}$ & YES & $\mathrm{NO}$ & $\mathrm{NO}$ & $\mathrm{NO}$ & $\mathrm{NO}$ & YES & $\mathrm{NO}$ & $\mathrm{NO}$ & $\mathrm{NO}$ & $\mathrm{NO}$ & YES \\
\hline
\end{tabular}


Table A3. Employment and Technology Spending - Size as Assets

Panel A report results from cross-sectional regressions of the natural log of technology and communication expense of banks as the dependent variable on the natural log of their total assets (Firm Size) for fthe sample years, as well as a pooled panel regression with year fixed effects. The standard errors are computing using HC3 robust standard errors for the first four columns and are clustered at the firm-level at the last column. The standard errors are reported in parentheses. $t$-statistics based on standard errors are in brackets. Panel B reports the results from panel regressions where the dependent variables are residual of bank employment and the independent variables are their previous-year residual technology spending as well as other firm characteristics. The coefficients on variables of years are suppressed from reporting. The standard errors are clustered at the firm-level and reported in parentheses. $t$-statistics based on robust standard errors are in brackets. All variables are defined in Table A1 in the appendix. To reduce noises in the analysis, firms with fewer than five consecutive years of technology expense and total assets information are excluded. Variables have been winsorized at the $1 \%$ and $99 \%$ tails of the distributions to avoid the influence of extreme observations.

Panel A. Technology Spending and Firm Size

\begin{tabular}{lccccc}
\hline & $(1)$ & $(2)$ & $(3)$ & $(4)$ & $(5)$ \\
Variables & 2000 & 2005 & 2010 & 2015 & Pooled \\
\hline \multirow{2}{*}{ Firm Size } & & & & & \\
& $0.828 * * *$ & $0.855^{* * *}$ & $0.658 * * *$ & $0.782 * * *$ & $0.767 * * *$ \\
& $(0.027)$ & $(0.027)$ & $(0.029)$ & $(0.024)$ & $(0.022)$ \\
Constant & {$[31.10]$} & {$[31.52]$} & {$[22.35]$} & {$[32.26]$} & {$[35.14]$} \\
& $-3.576 * * *$ & $-4.035 * * *$ & $-2.213 * * *$ & $-3.053 * * *$ & $-3.294 * * *$ \\
& $(0.126)$ & $(0.140)$ & $(0.138)$ & $(0.130)$ & $(0.097)$ \\
& {$[-28.28]$} & {$[-28.91]$} & {$[-16.03]$} & {$[-23.41]$} & {$[-34.13]$} \\
Observations & 301 & 426 & 474 & 500 & 7,758 \\
Firms & 301 & 426 & 474 & 500 & 780 \\
$R$-squared & 0.818 & 0.800 & 0.655 & 0.777 & 0.760 \\
Year FE & NO & NO & NO & NO & YES \\
\hline
\end{tabular}




\begin{tabular}{|c|c|c|c|c|}
\hline \multicolumn{5}{|c|}{ Panel B. Firm Size Employment and Technology Spending } \\
\hline \multirow{3}{*}{ Variables } & $(1)$ & $(2)$ & $(3)$ & (4) \\
\hline & Number of & Staff & Number of & Staff \\
\hline & & Expense & Employees & Expense \\
\hline \multirow[t]{3}{*}{ Technology Spending, $t-1$} & $0.124 * * *$ & $0.123 * * *$ & $0.109 * * *$ & $0.110 * * *$ \\
\hline & $(0.017)$ & $(0.014)$ & $(0.018)$ & $(0.015)$ \\
\hline & [7.32] & {$[8.85]$} & {$[5.95]$} & [7.36] \\
\hline \multirow[t]{3}{*}{ Market to Book, $t-1$} & & & -0.008 & -0.006 \\
\hline & & & $(0.009)$ & $(0.009)$ \\
\hline & & & {$[-0.83]$} & {$[-0.61]$} \\
\hline \multirow[t]{3}{*}{ Leverage, $t-1$} & & & -0.002 & -0.001 \\
\hline & & & $(0.002)$ & $(0.002)$ \\
\hline & & & {$[-0.83]$} & {$[-0.66]$} \\
\hline \multirow[t]{3}{*}{ Return on Assets, $t-1$} & & & $-1.647 * * *$ & $-1.859 * * *$ \\
\hline & & & $(0.436)$ & $(0.471)$ \\
\hline & & & {$[-3.78]$} & {$[-3.95]$} \\
\hline \multirow[t]{3}{*}{ Non-Interest Income, $t$ - 1} & & & $0.592 * * *$ & $0.623 * * *$ \\
\hline & & & $(0.086)$ & $(0.085)$ \\
\hline & & & {$[6.85]$} & {$[7.31]$} \\
\hline \multirow[t]{3}{*}{ Tier 1 Capital Ratio, $t-1$} & & & 0.060 & 0.272 \\
\hline & & & $(0.212)$ & $(0.194)$ \\
\hline & & & {$[0.28]$} & {$[1.40]$} \\
\hline \multirow[t]{3}{*}{ Constant } & -0.004 & 0.002 & -0.072 & $-0.097 * *$ \\
\hline & $(0.014)$ & $(0.013)$ & $(0.051)$ & $(0.043)$ \\
\hline & {$[-0.27]$} & {$[0.13]$} & {$[-1.40]$} & {$[-2.26]$} \\
\hline Observations & 6,526 & 7,162 & 5,573 & 6,084 \\
\hline Firms & 745 & 781 & 708 & 743 \\
\hline$R$-squared & 0.069 & 0.064 & 0.135 & 0.141 \\
\hline Size $t$ Effects & YES & YES & YES & YES \\
\hline Firm FE & YES & YES & YES & YES \\
\hline Year FE & YES & YES & YES & YES \\
\hline
\end{tabular}


Table A4. Employment and Technology Spending - Size as Total Loans and Deposits Panel A report results from cross-sectional regressions of the natural log of technology and communication expense of banks as the dependent variable on the natural log of their total loans and deposits (Firm Size) for fthe sample years, as well as a pooled panel regression with year fixed effects. The standard errors are computing using $\mathrm{HC} 3$ robust standard errors for the first four columns and are clustered at the firm-level at the last column. The standard errors are reported in parentheses. $t$-statistics based on standard errors are in brackets. Panel $B$ reports the results from panel regressions where the dependent variables are residual of bank employment and the independent variables are their previous-year residual technology spending as well as other firm characteristics. The coefficients on variables of years are suppressed from reporting. The standard errors are clustered at the firm-level and reported in parentheses. $t$-statistics based on robust standard errors are in brackets. All variables are defined in Table A1 in the appendix. To reduce noises in the analysis, firms with fewer than five consecutive years of technology expense and total assets information are excluded. Variables have been winsorized at the $1 \%$ and $99 \%$ tails of the distributions to avoid the influence of extreme observations.

Panel A. Technology Spending and Firm Size

\begin{tabular}{lccccc}
\hline \multirow{5}{*}{ Variables } & $(1)$ & $(2)$ & $(3)$ & $(4)$ & $(5)$ \\
& 2000 & 2005 & 2010 & 2015 & Pooled \\
Firm Size & $1.029 * * *$ & $0.966 * * *$ & $0.988 * * *$ & $0.930 * * *$ & $0.963 * * *$ \\
& $(0.023)$ & $(0.026)$ & $(0.023)$ & $(0.023)$ & $(0.019)$ \\
& {$[45.57]$} & {$[36.98]$} & {$[43.23]$} & {$[41.19]$} & {$[49.52]$} \\
Constant & $-7.131 * * *$ & $-6.739 * *$ & $-6.701 * * *$ & $-6.173 * * *$ & $-6.657 * * *$ \\
& $(0.157)$ & $(0.186)$ & $(0.170)$ & $(0.175)$ & $(0.130)$ \\
& {$[-45.32]$} & {$[-36.22]$} & {$[-39.39]$} & {$[-35.31]$} & {$[-51.27]$} \\
& & & & & \\
\hline Observations & 318 & 447 & 491 & 500 & 8,030 \\
Firms & 318 & 447 & 491 & 500 & 781 \\
$R$-squared & 0.881 & 0.835 & 0.834 & 0.837 & 0.845 \\
Year FE & $\mathrm{NO}$ & $\mathrm{NO}$ & $\mathrm{NO}$ & $\mathrm{NO}$ & YES \\
\hline
\end{tabular}




\begin{tabular}{|c|c|c|c|c|}
\hline \multicolumn{5}{|c|}{ Panel B. Firm Size Employment and Technology Spending } \\
\hline \multirow[b]{2}{*}{ Variables } & & (2) & (3) & (4) \\
\hline & $\begin{array}{l}\text { Number of } \\
\text { Employees }\end{array}$ & Staff Expense & $\begin{array}{l}\text { Number of } \\
\text { Employees }\end{array}$ & Staff Expense \\
\hline \multirow[t]{3}{*}{ Technology Spending, $t-1$} & $0.134 * * *$ & $0.136 * * *$ & $0.110 * * *$ & $0.112 * * *$ \\
\hline & $(0.016)$ & $(0.014)$ & $(0.016)$ & $(0.014)$ \\
\hline & {$[8.36]$} & [9.82] & [6.69] & [8.04] \\
\hline \multirow[t]{3}{*}{ Market to Book, $t-1$} & & & -0.010 & -0.014 \\
\hline & & & $(0.009)$ & $(0.009)$ \\
\hline & & & {$[-1.04]$} & {$[-1.58]$} \\
\hline \multirow[t]{3}{*}{ Leverage, $t-1$} & & & 0.000 & 0.001 \\
\hline & & & $(0.001)$ & $(0.001)$ \\
\hline & & & {$[0.18]$} & {$[0.59]$} \\
\hline \multirow[t]{3}{*}{ Return on Assets, $t-1$} & & & $-2.073 * * *$ & $-2.216^{* * *}$ \\
\hline & & & $(0.478)$ & $(0.505)$ \\
\hline & & & {$[-4.34]$} & {$[-4.39]$} \\
\hline \multirow[t]{3}{*}{ Non-Interest Income, $t-1$} & & & $0.699 * * *$ & $0.749 * * *$ \\
\hline & & & $(0.088)$ & $(0.089)$ \\
\hline & & & [7.90] & [8.38] \\
\hline \multirow[t]{3}{*}{ Tier 1 Capital Ratio, $t-1$} & & & $0.614 * * *$ & $0.850 * * *$ \\
\hline & & & $(0.178)$ & $(0.175)$ \\
\hline & & & {$[3.45]$} & [4.84] \\
\hline \multirow[t]{3}{*}{ Constant } & -0.012 & -0.009 & $-0.177 * * *$ & $-0.203 * * *$ \\
\hline & $(0.014)$ & $(0.014)$ & $(0.038)$ & $(0.035)$ \\
\hline & {$[-0.83]$} & {$[-0.66]$} & {$[-4.72]$} & {$[-5.73]$} \\
\hline Observations & 6,526 & 7,162 & 5,573 & 6,084 \\
\hline Firms & 745 & 781 & 708 & 743 \\
\hline$R$-squared & 0.076 & 0.069 & 0.167 & 0.180 \\
\hline Size $t$ Effects & YES & YES & YES & YES \\
\hline Firm FE & YES & YES & YES & YES \\
\hline Year FE & YES & YES & YES & YES \\
\hline
\end{tabular}




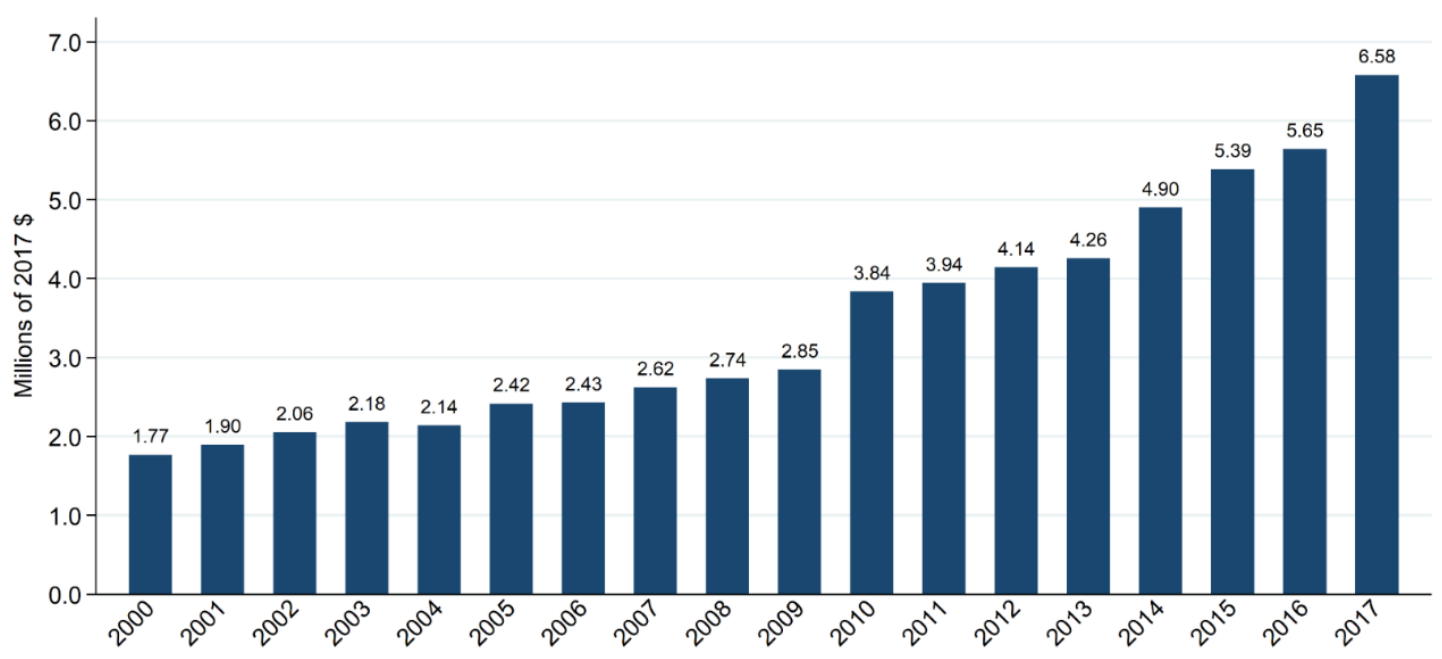

Figure B1. Technology Spending Trends

This figure illustrates the trends (medians) of technology and communication expense of banks in the sample that record technology and communication expense in each year during 2000-2017. There is a total of 97 firms. Technology and communication expense is converted to 2017 dollars using the GDP deflator. All variables are defined in Table A1 in the appendix. To reduce noises in the analysis, firms with fewer than five consecutive years of technology expense and total assets information are excluded. Variables have been winsorized at the $1 \%$ and $99 \%$ tails of the distributions to avoid the influence of extreme observation 


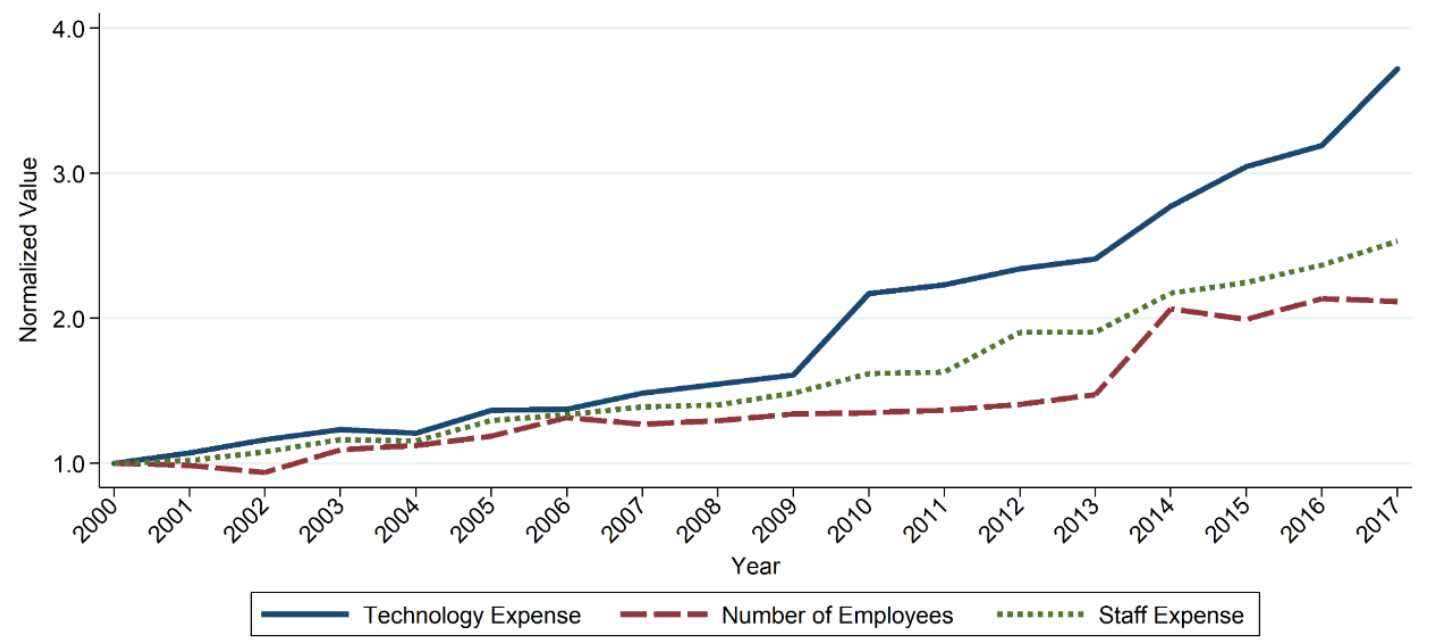

Figure B2. Employment and Technology Spending Over Time

This figure illustrates the evolution of the median of technology and communication expense, the number of employees and staff expense of banks in the sample that record technology and communication expense in each year during 2000-2017. There is a total of 97 firms. All values are normalized to equal one in the year 2000. Monetary variables are converted to 2017 dollars using the GDP deflator. All variables are defined in Table A1 in the appendix. To reduce noises in the analysis, firms with fewer than five consecutive years of technology expense and total assets information are excluded. Variables have been winsorized at the $1 \%$ and $99 \%$ tails of the distributions to avoid the influence of extreme observations. 
VITA

\section{ZIFENG FENG}

2005

B.A. in Public Administration

Peking University

Beijing, China

2011

Master of Business Administration

Jinan University

Guangdong, China

2014

M.Sc. in Real Estate

Florida International University

Miami, Florida

$2014-2019$

Doctoral Student

Florida International University

Miami, Florida

\section{PUBLICATIONS AND PRESENTATIONS}

"REITs' Operational Efficiency and Shareholders' Value" with Eli Beracha and William G. Hardin III, Journal of Real Estate Research, Accepted.

"REIT Operational Efficiency: Performance, Risk, and Return" with Eli Beracha and William G. Hardin III, Journal of Real Estate Finance and Economics, 58(3), 408-437.

"Technology Investment, Firm Performance and Market Value: Evidence from Banks" With Zhonghua Wu, Presented at 2018 Community Banking Research and Policy Conference

"Bank Technology: Productivity and Employment" with Zhonghua Wu, Presented at 2018 Financial Management Association International (FMA) Annual Meeting, and 2018 Southern Finance Association (SFA) Annual Meeting

"Employee Productivity and REIT Performance" with William G. Hardin III and Zhonghua Wu, Presented at 2017 American Real Estate Society (ARES) Annual Meeting

"REIT Executive Compensation and Firm Risks" with William G. Hardin III and Zhonghua Wu, Presented at 2017 American Real Estate Society (ARES) Annual Meeting, and 2018 American Real Estate \& Urban Economics Association (AREUEA-ASSA) Annual Meeting 